\title{
Study of the Ds+ to K+K-e+ nu Decay Channel with the Babar Experiment
}

\author{
Justine Serrano
}

SLAC-R-903

Prepared for the Department of Energy

under contract number DE-AC02-76SF00515

Printed in the United States of America. Available from the National Technical Information Service, U.S. Department of Commerce, 5285 Port Royal Road, Springfield, VA 22161. 
This document, and the material and data contained therein, was developed under sponsorship of the United States Government. Neither the United States nor the Department of Energy, nor the Leland Stanford Junior University, nor their employees, nor their respective contractors, subcontractors, or their employees, makes an warranty, express or implied, or assumes any liability of responsibility for accuracy, completeness or usefulness of any information, apparatus, product or process disclosed, or represents that its use will not infringe privately owned rights. Mention of any product, its manufacturer, or suppliers shall not, nor is it intended to, imply approval, disapproval, or fitness of any particular use. A royalty-free, nonexclusive right to use and disseminate same of whatsoever, is expressly reserved to the United States and the University. 


\title{
Université Paris XI UFR scientifique d'Orsay
}

\section{THĖSE}

présentée le 14 avril 2008

par

\section{Justine SERRANO}

\author{
pour obtenir le grade de \\ DOCTEUR EN SCIENCES \\ DE L'UNIVERSITÉ PARIS XI ORSAY
}
Étude de la désintégration $D_{s}^{+} \rightarrow K^{+} K^{-} e^{+} \nu_{e}$ dans l'expérience BABAR
Study of the $D_{s}^{+} \rightarrow K^{+} K^{-} e^{+} \nu_{e}$ decay channel with the BABAR experiment

soutenue devant la commission d'examen composée de:

$\begin{array}{llll}\text { Mme } & \text { V. } & \text { LUTH } & \text { Rapporteur } \\ \text { M. } & \text { O. } & \text { PẼNE } & \\ \text { Mme } & \text { S. } & \text { ROSIER LEES } & \text { Rapporteur } \\ \text { M. } & \text { P. } & \text { ROUDEAU } & \text { Directeur de thèse } \\ \text { M. } & \text { P. } & \text { SCHWEMLING } & \\ \text { M. } & \text { G. } & \text { WORMSER } & \text { Président }\end{array}$




\section{Contents}

$\begin{array}{ll}\text { Introduction } & 11\end{array}$

1 Theoretical background $\quad 13$

1.1 The Standard Model in the fermion sector . . . . . . . . . . . 13

1.1.1 Weak decays in the standard model . . . . . . . . . . . . . 14

1.1 .2 The CKM Matrix . . . . . . . . . . . . . 17

1.1 .3 The Unitarity Triangle . . . . . . . . . . . . . . 18

1.2 Dynamics of Semileptonic Decays . . . . . . . . . . . . . . 23

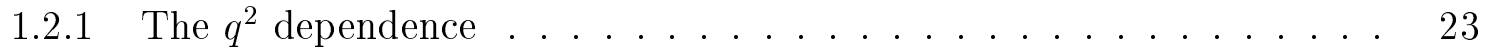

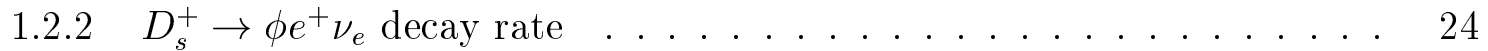

1.2.3 The general $D_{s}^{+} \rightarrow K^{+} K^{-} e^{+} \nu_{e}$ decay rate $\ldots \ldots \ldots . \ldots 29$

1.2.4 The 4-dimensionnal $D_{s}^{+} \rightarrow K^{+} K^{-} e^{+} \nu_{e}$ decay rate . . . . . . . 34

1.3 Form Factors in heavy hadron semileptonic decays . . . . . . . . . . . . 37

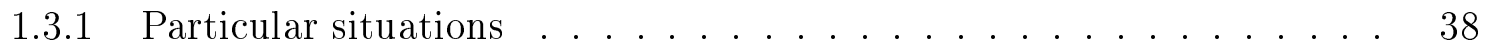

1.3.2 Different theoretical approaches to determine hadronic form factors . 44

1.3.3 Different ansatze to parameterize hadronic form factors . . . . . . . . 48

2 The BABAR Experiment $\quad 53$

2.1 The accelerator PEP-II . . . . . . . . . . . . . 54

2.2 The BABAR detector . . . . . . . . . . . . . . . . 57

2.2.1 The Silicon Vertex Tracker . . . . . . . . . . . . 58 
2.2 .2 The Drift Chamber . . . . . . . . . . . . . . . . 62

2.2 .3 The Cherenkov detector $\ldots \ldots \ldots \ldots \ldots$

2.2.4 The Electromagnetic Calorimeter . . . . . . . . . . . . . 71

2.2.5 The Instrumented Flux Return $\ldots \ldots \ldots \ldots$. . . . . . . 73

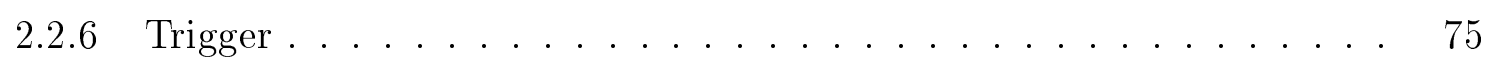

2.3 Particle identification . . . . . . . . . . . . . . . 75

2.3.1 Charged particle reconstruction $\ldots \ldots \ldots \ldots \ldots$

2.3 .2 Neutral particles reconstruction . . . . . . . . . . . . 77

2.3 .3 Particle identification . . . . . . . . . . . . . . . 77

$\begin{array}{lll}3 & \text { Event reconstruction and background rejection } & 81\end{array}$

3.1 Analysis method and data sample . . . . . . . . . . . . 81

3.1 .1 Analysis method $\ldots \ldots \ldots \ldots \ldots \ldots$

$3.1 .2 \quad$ Data sample . . . . . . . . . . . . . . . . 82

3.2 Event selection . . . . . . . . . . . . . . . . . . . . 83

$3.2 .1 \quad$ Skim preselection $\ldots \ldots \ldots \ldots \ldots \ldots \ldots$

3.2 .2 Global selection criteria $\ldots \ldots \ldots \ldots \ldots$. . . . . . . . 84

3.3 Event reconstruction $\ldots \ldots \ldots \ldots \ldots \ldots \ldots \ldots$

3.4 Background rejection $\ldots \ldots \ldots \ldots \ldots$

3.4 .1 Fisher analysis method . . . . . . . . . . . . . . 87

3.4.2 Rejection of $B \bar{B}$ events . . . . . . . . . . . . . . . . . . 89

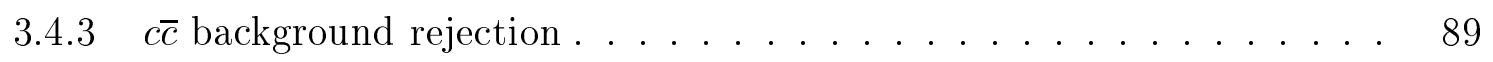

3.4.4 Rejection of gamma conversion $\ldots \ldots \ldots \ldots \ldots$

3.4.5 Background composition . . . . . . . . . . . . . . . . 91

3.5 Measurement of decay characteristics and reconstruction efficiency _ . . . 93

$\begin{array}{lll}4 & \text { Adjustements to the simulation } & 97\end{array}$ 
$4.1 D_{s}^{+} \rightarrow \phi \pi^{+}$events control sample $\ldots \ldots \ldots \ldots \ldots \ldots \ldots$

4.1.1 Variables used in the Fisher discriminants . . . . . . . . . . . . . 99

$4.1 .2 \quad D_{s}^{+}$direction and missing energy $\ldots \ldots \ldots \ldots \ldots$. . . . . . 104

4.2 Study of events with additional electrons or large charge imbalance . . . . 110

4.2.1 Events with an additional electron . . . . . . . . . . . . 110

4.2.2 Events with bad charge imbalance . . . . . . . . . . . . . . 114

4.3 Background adjustements . . . . . . . . . . . . . . . 117

4.3.1 Fisher variables for the $B \bar{B}$ background . . . . . . . . . . . 117

4.3.2 Fisher variables for the $c \bar{c}$ background $\ldots \ldots \ldots \ldots \ldots$

$4.3 .3 \quad K^{ \pm}$production in charm events . . . . . . . . . . . . 125

4.3.4 Charm peaking background . . . . . . . . . . . . . . . 134

4.3.5 The non-peaking background overall rate . . . . . . . . . . . 140

4.4 Summary of corrections applied to the Monte Carlo simulation . . . . . . . 147

4.5 Corrected distributions . . . . . . . . . . . . . . . . . 147

$\begin{array}{lll}5 & \text { Determination of form factors parameters } & 151\end{array}$

5.1 Fit procedure . . . . . . . . . . . . . . . . . 151

5.2 Fit quality . . . . . . . . . . . . . . . . 153

5.3 Parameters sensitivity $\ldots \ldots \ldots \ldots \ldots \ldots \ldots$

5.4 Results on simulated events . . . . . . . . . . . . . . . 159

5.4 .1 Toy simulations . . . . . . . . . . . . . . . . . . 159

5.4 .2 Analysis of fully simulated events . . . . . . . . . . . . . 162

6 Results 165

6.1 Fit values . . . . . . . . . . . . . . . . . 165

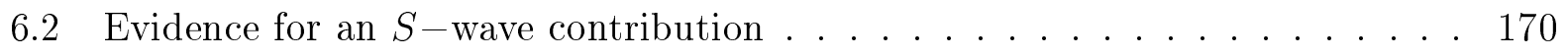

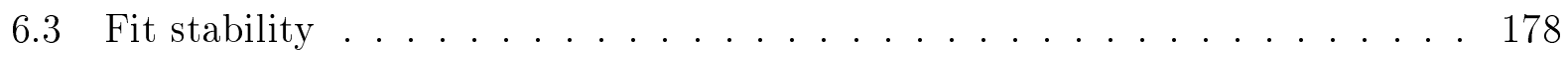


$\begin{array}{llr}7 & \text { Systematic uncertainties } & 181\end{array}$

7.1 Signal Monte Carlo corrections _ . . . . . . . . . . . . . . . . 182

7.2 Background Monte Carlo corrections _ . . . . . . . . . . . . . . . 184

7.2.1 Fragmentation correction on the $c \bar{c}$ background . . . . . . . . 184

$7.2 .2 \quad B \bar{B}$ background correction . . . . . . . . . . . . . . . 185

7.2.3 Continuum background under the $\phi$ meson . . . . . . . . . . . 185

7.2 .4 Peaking background correction $\ldots \ldots \ldots \ldots \ldots$

7.2.5 Summary of background MC corrections systematics . . . . . . 186

7.3 Fitting procedure $\ldots \ldots \ldots \ldots \ldots \ldots \ldots$

7.4 Particle identification efficiencies . . . . . . . . . . . . . 186

7.5 Radiative events . . . . . . . . . . . . . . . . . . 187

$7.6 \quad S$-wave parameterization $\ldots \ldots \ldots \ldots \ldots \ldots \ldots \ldots$

7.7 Summary on systematic effects . . . . . . . . . . . . . . 188

8 Decay rate measurement $\quad 195$

8.1 Branching fraction measurement . . . . . . . . . . . . . . 196

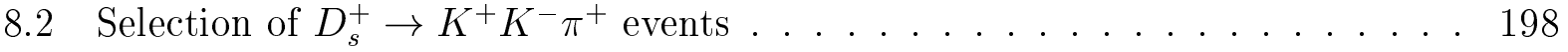

8.3 Selection of $D_{s}^{+} \rightarrow K^{+} K^{-} e^{+} \nu_{e}$ events $\ldots \ldots \ldots \ldots$

8.4 Measured value of $R_{D s}$ and statistical uncertainty $\ldots \ldots \ldots 201$

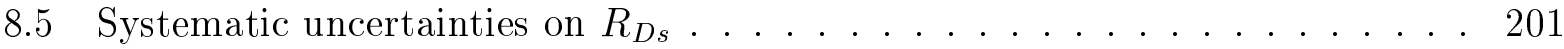

8.5.1 Cut on the Fisher discriminant variables . . . . . . . . . . . 202

8.5.2 Number of events $D_{s}^{+} \rightarrow K^{+} K^{-} e^{+} \nu_{e} \ldots \ldots \ldots \ldots 20 \ldots$

8.5.3 Mass constrained fit probability . . . . . . . . . . . . . . 203

8.5.4 Particle identification . . . . . . . . . . . . . . . . . 203

8.5.5 Radiative events . . . . . . . . . . . . . . . . . . 203

8.5.6 Signal events evaluation . . . . . . . . . . . . . . . . . 203

8.5.7 Summary on systematic errors . . . . . . . . . . . . . . . 204 
8.6 Decay rate measurement and value of the hadronic form factor at $q^{2}=0 \quad$. 204

9 Physics interpretations $\quad 207$

9.1 Form factors parameterization . . . . . . . . . . . . 207

9.2 Comparison with other experimental results . . . . . . . . . . . 209

9.3 Comparison with theoretical calculations . . . . . . . . . . 213

9.4 Present measurements and HQET . . . . . . . . . . . . . 218

9.4.1 Determination of QCD corrections .............. 218

9.4.2 Determination of $\bar{\Lambda} / 2 m_{s} \ldots \ldots \ldots \ldots \ldots$

9.4.3 Evaluation of the corrections to $f_{+}\left(q_{\max .}^{2}\right)$ and $A_{2}\left(q_{\max .}^{2}\right) \ldots \ldots 221$

9.5 Experimental results on charm semileptonic decays branching fractions . . . 223

9.5.1 Inclusive results . . . . . . . . . . . . . 223

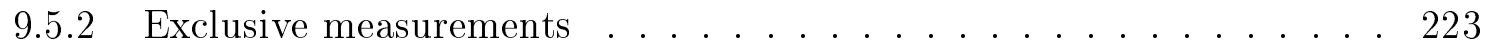

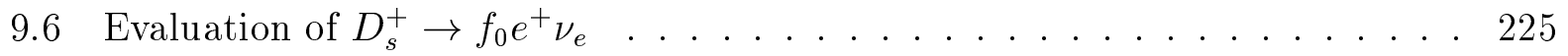

$\begin{array}{ll}\text { Conclusion } & 227\end{array}$

$\begin{array}{ll}\text { Remerciements } & 233\end{array}$ 


\section{Résumé}

Les désintégrations semileptoniques du charme permettent une validation des calculs de QCD sur réseau grâce à la mesure de facteurs de forme hadroniques qui décrivent l'effet de l'interaction forte dans ces réactions. La précision de ces calculs joue un rôle crucial dans l'amélioration des tests du modèle standard dans le domainde des saveurs. Cette thèse présente une étude du canal $D_{s}^{+} \rightarrow K^{+} K^{-} e^{+} \nu_{e}$, utilisant $214 f b^{-1}$ de données collectées par l'expérience Babar. Pour les évènements ayant une masse $K^{+} K^{-}$dans l'intervalle entre $1.01 \mathrm{GeV} / \mathrm{c}^{2}$ et $1.03 \mathrm{GeV} / \mathrm{c}^{2}$, le $\phi \rightarrow K^{+} K^{-}$est le canal dominant. En utilisant le modèle des pôles simples pour paramétrer la dépendance en $q^{2}\left(=\left(p_{e}+p_{\nu}\right)^{2}\right)$ des facteurs de forme $V\left(q^{2}\right), A_{1}\left(q^{2}\right)$ et $A_{2}\left(q^{2}\right)$, les rapports suivant sont mesurés à $q^{2}=0: r_{V}=V(0) / A_{1}(0)=$ $1.868 \pm 0.061 \pm 0.079, r_{2}=A_{2}(0) / A_{1}(0)=0.763 \pm 0.072 \pm 0.062$. La masse du pôle du facteur de forme axial-vecteur est également obtenue: $m_{A}=\left(2.30_{-0.18}^{+0.24} \pm 0.21\right) \mathrm{GeV} / \mathrm{c}^{2}$. Dans le même intervalle de masse, le rapport d'embranchement relatif au canal $D_{s}^{+} \rightarrow \phi \pi^{+}$, est mesuré, et la normalisation absolue du facteur de forme $A_{1}(0)$ en est déduite: $A_{1}\left(q^{2}=\right.$ $0)=0.605 \pm 0.012 \pm 0.018 \pm 0.018$. Les erreurs mentionnées correspondent aux incertitudes statistique, systématique et à celle due aux mesures extérieures à cette analyse. Enfin, le système $K^{+} K^{-}$en onde $\mathrm{S}$, pouvant être issu du $f_{0}$, est étudié à travers son interférence avec le $\phi$. Une composante d'onde $\mathrm{S}$ est observée dans ce canal pour la première fois à plus de $5 \sigma$. 


\section{Abstract}

Charm semileptonic decays allow a validation of lattice QCD calculations through the measurement of the hadronic form factors, which caracterize the effect of strong interaction in these reactions. The accuracy of such calculations is crucial for the improvement of the test of the standard model in flavour physics. This thesis presents a study of the $D_{s}^{+} \rightarrow$ $K^{+} K^{-} e^{+} \nu_{e}$ channel using $214 f b^{-1}$ recorded by de Babar experiment. For events with a $K^{+} K^{-}$mass in the range between $1.01 \mathrm{GeV} / \mathrm{c}^{2}$ and $1.03 \mathrm{GeV} / \mathrm{c}^{2}$, the $\phi \rightarrow K^{+} K^{-}$is the dominant component. Using the simple pole model to parameterize the $q^{2}$ dependence of the form factors $-V\left(q^{2}\right), A_{1}\left(q^{2}\right)$ and $A_{2}\left(q^{2}\right)$ - the following ratios are measured at $q^{2}=0$ : $r_{V}=V(0) / A_{1}(0)=1.868 \pm 0.061 \pm 0.079, r_{2}=A_{2}(0) / A_{1}(0)=0.763 \pm 0.072 \pm 0.062$. The mass pole of the axial-vector form factor is also obtained: $m_{A}=\left(2.30_{-0.18}^{+0.24} \pm 0.21\right) \mathrm{GeV} / \mathrm{c}^{2}$. In the same mass range, the semileptonic branching fraction, relative to the $D_{s}^{+} \rightarrow \phi \pi^{+}$channel, is measured, and the abolute normalisation of the axial-vector form factor is extracted: $A_{1}\left(q^{2}=0\right)=0.605 \pm 0.012 \pm 0.018 \pm 0.018$. The stated errors refer to the statistical, systematic and errors from external inputs, respectively. An $\mathrm{S}$ wave component in the $K^{+} K^{-}$system, possibly originating from a $f_{0}$, is also studied through its interference with the $\phi$. An S wave component is observed for the first time in this decay channel with a $5 \sigma$ significance. 


\section{Introduction}

The capability of the Standard Model (SM) in describing the interactions between elementary particles is at present well established. This model has been widely tested, in particular with precision measurements in the flavour sector, thanks to particle accelerator experiments. In this framework, the main goal of $B$ factories was to find out whether the SM was able to describe the $\mathrm{CP}$ violation observed in nature. Until now, they did not demonstrate any deviation from theory and fits of the Unitarity Triangle, characterizing the amplitude of CP violation, are consistent.

On the other hand, we know that the Standard Model is incomplete. For example it neither explains the origin of neutrino masses nor the matter-antimatter asymmetry of the Universe. New physics, appearing through extensions of the SM, is needed for that. In order to find effects from new physics, it is important to improve the accuracy of the SM tests. This can be done, in particular, through a better knowledge of the effects of the strong interaction thanks to experimental precision measurements and theoretical calculations such as lattice QCD, that uses directly the first principles of this interaction.

Semileptonic decays play a special role in this domain as they are parameterized by the product of an element of the CKM matrix and form factors in which are hidden the QCD effects. In case of charm meson decays, contrary to $B$ meson, the value of the matrix element is known from other constraints and we can study directly the form factors.

The $D_{s}^{+} \rightarrow \phi e^{+} \nu_{e}$ is an ideal place to study charm meson semileptonic decays into a vector meson as the $\phi$ meson is a narrow resonance. In addition, as only $c$ and $s$ valence quarks are involved in this process, one expects that evaluations from lattice QCD for this decay channel, when available, will be more accurate than for non-strange charm mesons. Experimentally, the study of $D_{s}$ decays is more difficult than for non-strange $D$ meson as these states are produced with only $\sim 10 \%$ probability by hadronization of a charm quark. In effect, previous experiments have analyzed rather low statistics samples of $D_{s}^{+} \rightarrow \phi e^{+} \nu_{e}$ semileptonic decays. The largest sample corresponding to $\sim 560$ events was collected by the FOCUS collaboration and results were published in 2004. The CLEO-c experiment which collects events close to the threshold for $D_{s} \bar{D}_{s}^{(*)}$ production has not published yet results on 
$D_{s}$ semileptonic decays. Thanks to the large statistics of $c \bar{c}$ events produced at the energy of the $\Upsilon(4 S)$, for which the cross section is equal to $1.3 \mathrm{nb}$, the BABAR experiment can also be seen as a charm factory. The analysis presented in this thesis provides a breakthrough on this channel with a signal statistics multiplied by $\sim 50$ and a lower background. With this statistics it is also possible to study the eventual existence of an $S$-wave component in the $K^{+} K^{-}$system, through its interference with the $P$-wave (the $\phi$ meson). Only the FOCUS experiment had searched for this component. In the present analysis, systematics uncertainties have been also reduced at a level similar to the statistical accuracy. This has been obtained using various data samples to control detector performances, the production characteristics of the signal and the properties of the different background components.

In the first chapter, we described the theoretical framework needed to understand the kinematics of semileptonic decays. We detail the case of a meson decaying into two pseudo scalar particles, for which the dynamics is contained in three hadronic form factors. To determine the normalization and the variation with $q^{2}\left(q^{2}\right.$ is the mass squared of the $e \nu$ system) of these form factors, it is necessary to use models or more elaborate calculations from lattice QCD. These different approaches are reviewed in the last section of the first chapter. In the second chapter, the BABAR experiment is presented as well as some aspects of the particle identification used in this analysis. The third chapter presents the event selection and background rejection. In chapter 4, we explain the measurements needed to correct for differences between data and simulation for signal and background. The validation of the fit program used to extract the decay parameters is explained in the fifth chapter and results are given in chapter 6 . Systematic uncertainties are then explained in chapter 7 . In chapter 8 , we present the decay rate measurement which gives access to the absolute normalization of the form factors. Finally, the last chapter contains a discussion of the obtained results. They are compared with previous experimental results and different theoretical approaches. 


\section{Chapter 1}

\section{Theoretical background}

The rabbit-hole went straight on like a tunnel for some way, and then dipped suddenly down, so suddenly that Alice had not a moment to think about stopping herself before she found herself falling down a very deep well...

Lewis Carroll, Alice's Adventures in Wonderland.

In this chapter, we first introduce the standard model in the fermion sector, the CKM matrix and the unitarity triangle. We then detail the dynamics of semileptonic decays, focusing on the $D_{s}^{+} \rightarrow \phi e^{+} \nu_{e}$ and $D_{s}^{+} \rightarrow K^{+} K^{-} e^{+} \nu_{e}$ decay rates. In the last section, we give different theoretical approaches for the parameterization of the form factors.

\subsection{The Standard Model in the fermion sector}

The Standard Model [1] is the present theory which describes elementary particles and their interactions: weak, electromagnetic and strong. According to this model, matter is formed by two types of fundamental particles with spin 1/2 (particles with half integer spin are called fermions), leptons and quarks. Each type is divided into three families.

For the quarks:

$$
\left(\begin{array}{l}
u \\
d
\end{array}\right)\left(\begin{array}{l}
c \\
s
\end{array}\right)\left(\begin{array}{l}
t \\
b
\end{array}\right)
$$

For the leptons: 


$$
\left(\begin{array}{l}
e^{-} \\
\nu_{e}
\end{array}\right)\left(\begin{array}{c}
\mu^{-} \\
\nu_{\mu}
\end{array}\right)\left(\begin{array}{l}
\tau^{-} \\
\nu_{\tau}
\end{array}\right)
$$

The quarks have a fractionnary electric charge, which is $2 / 3$ for the up type quarks (up, charm, top) and $-1 / 3$ for the down type quarks (down, strange and bottom), while the leptons have an integer charge: -1 for the electron, muon and tau, and 0 for the neutrinos.

Interactions between the elementary fermions are carried by particles with integer spin, the gauge bosons. The vector of the electromagnetic interaction is the photon. It couples only to non-zero electric charge fermions. The bosons $W$ and $Z$ correspond to the weak interaction and couple to all fundamental fermions. The gluons, vector of the strong interaction, only couple to the quarks. Properties of these intermediate bosons are summarized in table 1.1. Because of the non abelian character of the weak and strong interactions, the weak bosons and the gluons couple also to themselves.

\begin{tabular}{|c|c|c|c|}
\hline Interaction & Bosons & Spin & Mass $\left(\mathrm{GeV} / \mathrm{c}^{2}\right)$ \\
\hline Weak & $W^{ \pm}$ & 1 & $80.403 \pm 0.029$ \\
\hline Weak & $Z^{0}$ & 1 & $91.1876 \pm 0.0021$ \\
\hline Electromagnetic & $\gamma$ & 1 & 0 \\
\hline Strong & $g$ & 1 & 0 \\
\hline
\end{tabular}

Table 1.1: Summary of the properties of the Intermediate bosons.

\subsubsection{Weak decays in the standard model}

The weak interaction plays an important role in the Standard Model because it is the only interaction that allows the coupling of quarks from different families. Weak decays can be divided into three classes according to the structure of the decay, which are illustrated in Figure 1.1. Figure 1.1-a, shows a leptonic decay, where the quarks from the hadron annihilate each other and only leptons are present in the final state; in semileptonic decays, both lepton and hadron are present in the final state (Figure 1.1-b); in hadronic decays, the $W$ decays into two quarks so there is only hadrons in the final state (Figure 1.1-c). These diagrams represent the interactions at quark level, but the reality is much more complex as quarks are bound inside hadrons by the strong force. Effects from strong interaction, described in the Standard Model by Quantum Chromodynamics (QCD), are usually difficult to predict in these decays because the strong coupling is too large and these effects cannot be calculated using perturbative methods.

From this situation arises the advantage of leptonic and semileptonic decays, for which the decay amplitude can be written as a product of the relatively simple leptonic current by 
the more complex hadronic current. As the strong interaction can be isolated, these decays provide a simple environment to study the effects of strong coupling.
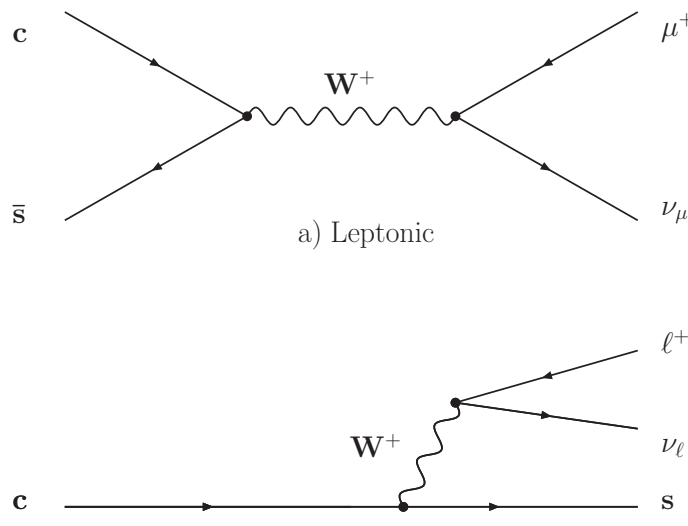

$\overline{\mathrm{q}}$

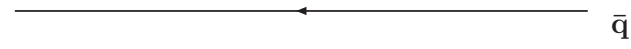

b) Semileptonic

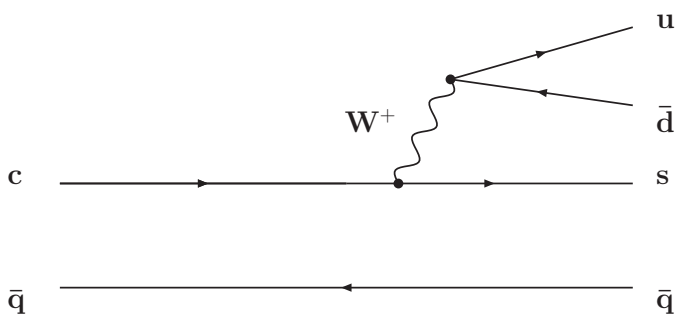

c) Hadronic

Figure 1.1: Examples of Feynam diagrams of charm meson decays: (a) leptonic decay, (b) semileptonic decay, (c) hadronic decay.

In the Standard Model, weak interaction is based on the gauge theory $S U(2)_{L} \otimes U(1)_{Y}$ where the index $L$ stands for left, since only the left handed particles are involved in the charged weak current. The interaction Lagrangian is expressed in terms of two operators: the Vector-Axial (V-A) charged weak current operator $J^{\mu}$, and the operator corresponding to the $W$ boson field, $W_{\mu}$ :

$$
\mathcal{L}_{c c}=-\frac{g}{\sqrt{2}}\left(J^{\mu} W_{\mu}^{\dagger}+J^{\mu \dagger} W_{\mu}\right)
$$

The $W_{\mu}$ operator annihilates a $W^{-}$boson or creates a $W^{+}$(the reverse is true for $\left.W_{\mu}^{\dagger}\right) . g$ is a coupling constant. The weak current operator $J^{\mu}$ which accounts for quark states is given by:

$$
J^{\mu}=\sum_{i, j} V_{i j} J_{i j}^{\mu}=\sum_{i, j} \bar{u}_{i} \gamma^{\mu} \frac{1}{2}\left(1-\gamma_{5}\right) V_{i j} d_{j}
$$


where the indices $i$ and $j$ run over the three generations (the field operator $u_{i}$ annihilates $u, c, t$ and the $d_{i}$ annihilates $d, s, b$, or create their antiparticles), and $V_{i j}$ is the element of the Cabibbo-Kobayashi-Maskawa (CKM) matrix [2,3]. This matrix is coming form the fact that the weak eigenstates $\left(d^{\prime}, s^{\prime}, b^{\prime}\right)$ are different from the mass eigenstates $(d, s, b)$, and it can be seen as the rotation that allows to pass from one basis to the other:

$$
\left(\begin{array}{c}
d^{\prime} \\
s^{\prime} \\
b^{\prime}
\end{array}\right)=\left(\begin{array}{ccc}
V_{u d} & V_{u s} & V_{u b} \\
V_{c d} & V_{c s} & V_{c b} \\
V_{t d} & V_{t s} & V_{t b}
\end{array}\right)\left(\begin{array}{c}
d \\
s \\
b
\end{array}\right)
$$

This matrix, through its non-diagonal terms, allows flavour changing transition between quarks.

Considering the example of the semileptonic decay $c \rightarrow s W^{+}\left(e^{+} \nu\right)$, from the Lagrangian defined in Eq. (1.1), the decay amplitude can be written:

$$
\mathcal{M}=\frac{g}{\sqrt{2}} \bar{u}_{c} V_{c s} \gamma^{\mu}\left(1-\gamma^{5}\right) u_{s} \frac{1}{M_{W}^{2}-q^{2}} \frac{g}{\sqrt{2}} \bar{u}_{e} \gamma^{\mu}\left(1-\gamma^{5}\right) u_{\nu}
$$

where $q^{2}=\left(P_{e}+P_{\nu}\right)^{2}$ is the squared mass of the virtual $W$. In this equation the quark current (first term) and the leptonic current (last term) are coupled through the propagator term $\frac{1}{M_{W}^{2}-q^{2}}$. When the involved energies in the decay are much less than the $W$ mass, $g$ is related to the Fermi constant $G_{F}$ by:

$$
G_{F}=\frac{g^{2}}{4 \sqrt{2} M_{W}^{2}}
$$

In this approximation, the general amplitude for semileptonic decays $M_{Q \bar{q}} \rightarrow X_{q^{\prime} \bar{q} l^{-} \bar{\nu}}$ can be written as:

$$
\mathcal{M}=-i \frac{G_{F}}{\sqrt{2}} V_{Q q^{\prime}} L^{\mu} H_{\mu}
$$

where the leptonic current is expressed in terms of the Dirac spinors $\bar{u}_{l}$ and $v_{\nu}$ :

$$
L^{\mu}=\bar{u}_{l} \gamma^{\mu}\left(1-\gamma^{5}\right) u_{\nu}
$$

and the hadronic current $H^{\mu}$ is related to the matrix element of the operator $J^{\mu}$ given in Eq. (1.6):

$$
H^{\mu}=<X\left|\bar{q}^{\prime} \gamma^{\mu}\left(1-\gamma^{5}\right) Q\right| M>
$$

The hadronic current contains all the effects from the strong interaction and cannot be calculated in a simple way. These effects are parameterized by means of form factors, that 
usually depend on $q^{2}$. Their origin and parameterization will be explained in more detail in section 1.3.

Leptonic decays are simpler than semileptonic ones because the effect of strong interactions can be parameterized in terms of a single quantity called the decay constant, which is proportional to the probability amplitude for the quarks to have zero separation. Whereas in semileptonic decays, $q^{2}$ can vary from 0 to $q_{m a x}^{2}=\left(M_{Q \bar{q}}-X_{q^{\prime} \bar{q}}\right)^{2}$, in leptonic decays $q^{2}$ is fixed to the mass squared of the decaying hadron. The amplitude of the leptonic decay $M_{Q \bar{q}} \rightarrow l^{-} \bar{\nu}$ is written:

$$
\mathcal{M}=-i \frac{G_{F}}{\sqrt{2}} V_{q Q} f_{M} L^{\mu} q_{\mu}
$$

where $q_{\mu}$ is the four momentum of the meson and $f_{M}$ the decay constant. Leptonic decays are in general less accessible experimentally than semileptonic decays because their branching fractions are smaller due to a term of helicity suppression in the decay rate, proportionnal to the square of the lepton mass.

\subsubsection{The CKM Matrix}

We have seen that the CKM matrix plays an important role in weak decays because it parameterizes the transitions between quarks. In the following, we introduce two standard parameterizations of this matrix. Within the Standard Model, its elements are input parameters and cannot be predicted. Nevertheless one can demonstrate [3] that the threegenerations matrix can be expressed in terms of three real numbers and one phase factor $e^{i \delta}$. A standard parameterization is given in [4] using the set of angles $\theta_{12}, \theta_{23}, \theta_{13}$ and $\delta$ :

$$
V_{\mathrm{CKM}}=\left(\begin{array}{ccc}
c_{12} c_{13} & s_{12} c_{13} & s_{13} e^{-i \delta} \\
-s_{12} c_{23}-c_{12} s_{23} s_{13} e^{i \delta} & c_{12} c_{23}-s_{12} s_{23} s_{13} e^{i \delta} & s_{23} c_{13} \\
s_{12} s_{23}-c_{12} c_{23} s_{13} e^{i \delta} & -s_{23} c_{12}-s_{12} c_{23} s_{13} e^{i \delta} & c_{23} c_{13}
\end{array}\right)
$$

where $c_{i j}=\cos \theta_{i j}$ and $s_{i j}=\sin \theta_{i j}(i, j=1,2,3$, are the family's index, $j>i)$. The phase $\delta$ is responsible of the fact that the CP symmetry is violated in the Standard Model. $c_{i j}$ and $s_{i j}$ can all be chosen to be positive, and $\delta$, from measurements of kaons decays, is restricted in the interval $0<\delta<\pi$. From experiment, it is known that $s_{13}$ is of the order of $10^{-3}$, so $c_{13}$ is really close to unity, and $s_{23}$ is of the order of $10^{-2}$.

Another parameterization, proposed by Wolfenstein, illustrates the hierarchy in the magnitude of the CKM elements [5]. It uses the set of parameters $A, \lambda, \rho$ and $\eta$ defined as $s_{12}=\lambda$, 
$s_{23}=A \lambda^{2}, s_{13} e^{-i \delta}=A \lambda^{3}(\rho-i \eta)$, and gives:

$$
V_{\mathrm{CKM}}=\left(\begin{array}{ccc}
1-\frac{\lambda^{2}}{2} & \lambda & A \lambda^{3}(\varrho-i \eta) \\
-\lambda & 1-\frac{\lambda^{2}}{2} & A \lambda^{2} \\
A \lambda^{3}(1-\varrho-i \eta) & -A \lambda^{2} & 1
\end{array}\right)+\mathcal{O}\left(\lambda^{4}\right)
$$

In this parameterization, the elements $V_{u s}$ and $V_{c d}$, being of the order of 0.2 are similar to $\lambda$, and the diagonal elements are very close to one. $V_{c b}$ and $V_{t s}$ are of the order of $4.10^{-2}$. $V_{u b}$ and $V_{t d}$ are of the order of $5 \cdot 10^{-3}$. The matrix is almost diagonal, so the coupling between quarks of the same family is close to unity, and is decreasing as the separation between family increases.

A more accurate expression of the Wolfenstein parameterization, valid at $\mathcal{O}\left(\lambda^{6}\right)$, gives [6]:

$$
\left(\begin{array}{ccc}
1-\frac{1}{2} \lambda^{2}-\frac{1}{8} \lambda^{4} & \lambda+\mathcal{O}\left(\lambda^{7}\right) & A \lambda^{3}(\rho-i \eta) \\
-\lambda+\frac{1}{2} A^{2} \lambda^{5}[1-2(\rho+i \eta)] & 1-\frac{1}{2} \lambda^{2}-\frac{1}{8} \lambda^{4}\left(1+4 A^{2}\right) & A \lambda^{2}+\mathcal{O}\left(\lambda^{8}\right) \\
A \lambda^{3}(1-\bar{\rho}-i \bar{\eta}) & -A \lambda^{2}+\frac{1}{2} A \lambda^{4}[1-2(\bar{\rho}+i \bar{\eta})] & 1-\frac{1}{2} A^{2} \lambda^{4}
\end{array}\right)
$$

where $\bar{\rho}$ and $\bar{\eta}$ are related to $\rho$ and $\eta$ by:

$$
\bar{\rho}=\rho\left(1-\frac{\lambda^{2}}{2}\right), \quad \bar{\eta}=\eta\left(1-\frac{\lambda^{2}}{2}\right) .
$$

\subsubsection{The Unitarity Triangle}

The unitarity of the CKM matrix allows to write nine equations relating its elements, in particular:

$$
V_{u d} V_{u b}^{*}+V_{c d} V_{c b}^{*}+V_{t d} V_{t b}^{*}=0 .
$$

Using the parameterization of Eq. (1.12), we can express the terms of this equation as function of $A, \lambda, \bar{\rho}$ and $\bar{\eta}$ :

$$
\begin{aligned}
V_{u d} V_{u b}^{*} & =A \lambda^{3}(\bar{\rho}+i \bar{\eta}), \\
V_{c d} V_{c b}^{*} & =-A \lambda^{3}, \\
V_{t d} V_{t b}^{*} & =A \lambda^{3}(1-\bar{\rho}-i \bar{\eta})
\end{aligned}
$$

The quantity $A \lambda^{3}$ can be factored out, and the geometrical representation of Eq. (1.14) is a triangle in the $(\bar{\rho}, \bar{\eta})$ plane, with summits at $\mathrm{C}(0,0), \mathrm{B}(1,0)$ and $\mathrm{A}(\bar{\rho}, \bar{\eta})$ (Figure 1.2). This triangle is called the Unitarity Triangle. It depends on two parameters $(\bar{\rho}, \bar{\eta})$, and with the two other parameters $\left|V_{u s}\right|$ and $\left|V_{c b}\right|$ (related to A and $\lambda$ ), it gives a complete description of the CKM matrix. One can note that if the $C P$ symmetry would have been conserved in the Standard Model, the triangle would have collapsed into a line. The area of the triangle is a 
direct measurement of $C P$ violation. Five other triangles can also be defined from equations similar to (1.14), but this is the only triangle characterizing the $C P$ violation in the $B_{d}$ sector having three sides of the same order $\left(\lambda^{3}\right)$.

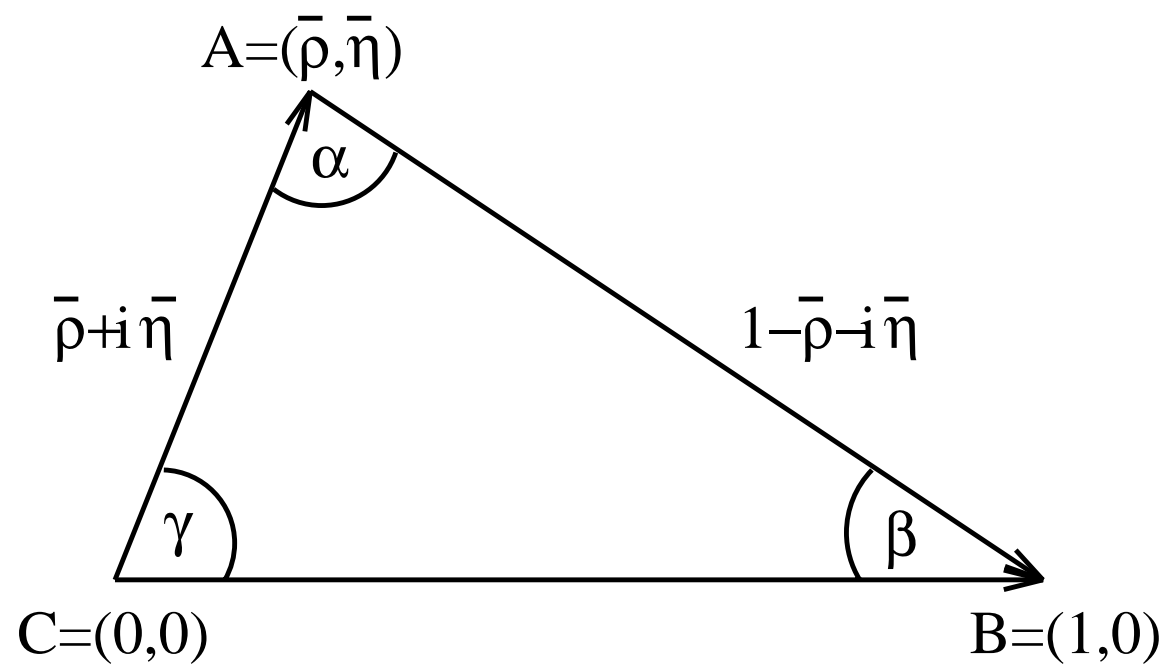

Figure 1.2: Unitarity Triangle in the $\bar{\rho}-\bar{\eta}$ plane.

The lengths $C A$ and $B A$, usually noted $R_{b}$ and $R_{t}$ are given by:

$$
\begin{aligned}
& \overline{C A}=R_{b}=\frac{\left|V_{u d} V_{u b}^{*}\right|}{\left|V_{c d} V_{c b}^{*}\right|}=\sqrt{\bar{\rho}^{2}+\bar{\eta}^{2}}=\left(1-\frac{\lambda^{2}}{2}\right) \frac{1}{\lambda}\left|\frac{V_{u b}}{V_{c b}}\right|, \\
& \overline{B A}=R_{t}=\frac{\left|V_{t d} V_{t b}^{*}\right|}{\left|V_{c d} V_{c b}^{*}\right|}=\sqrt{(1-\bar{\rho})^{2}+\bar{\eta}^{2}}=\frac{1}{\lambda}\left|\frac{V_{t d}}{V_{c b}}\right|
\end{aligned}
$$

The three angles are :

$$
\begin{aligned}
& \alpha=\arg \left(\frac{V_{t d} V_{t b}^{*}}{V_{u d} V_{u b}^{*}}\right), \beta=\arg \left(\frac{V_{c d} V_{c b}^{*}}{V_{t d} V_{t b}^{*}}\right), \\
& \gamma=\arg \left(\frac{V_{u d} V_{u b}^{*}}{V_{c d} V_{c b}^{*}}\right)=\pi-\alpha-\beta
\end{aligned}
$$

Thanks to the measurements of $C P$ violation for $B$ mesons at $B$-factories, we have now access to direct measurements of these angles. Several other physical quantities, obtained by experiments, can also be related to the Unitarity Triangle parameters, as we will explain below. The Standard Model predicts that all measurements should give compatible values for the apex determined by the $\bar{\rho}$ and $\bar{\eta}$ coordinates. The Unitarity Triangle provides thus an important way of testing the Standard Model in the fermion sector.

The indirect measurements are done through the following quantities: 
- The ratio $\frac{\left|V_{c b}\right|}{\left|V_{c b}\right|}:$

$$
\frac{\left|V_{u b}\right|}{\left|V_{c b}\right|}=\frac{\lambda}{1-\frac{\lambda^{2}}{2}} \sqrt{\bar{\rho}^{2}+\bar{\eta}^{2}} .
$$

The quantities $\left|V_{c b}\right|$ and $\left|V_{u b}\right|$ can be measured through the decay rate of inclusive and exclusive semileptonic $b$-decays $b \rightarrow c l \nu$ and $b \rightarrow u l \nu$. Both methods use a specific theoretical framework: inclusive measurements rely on the Operator Product Expansion (OPE), and exclusive measurements need a description of the form factors. Different models of form factors will be explained in section 1.3. It is important to note that the uncertainties on exclusive measurements of $\left|V_{c b}\right|$ and $\left|V_{u b}\right|$ are dominated by the theoretical uncertainties on the form factors models.

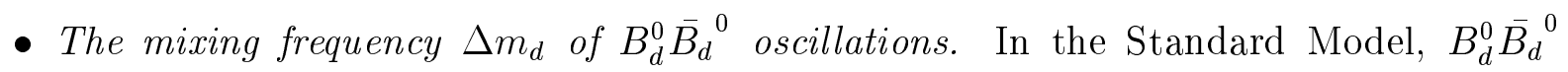
oscillations occur through a box diagram with a loop containing a $W$ and an up type quark (the top quark gives the dominant contribution). The oscillation frequency is determined by the mass difference between the $B_{H}^{0}$ and $B_{L}^{0}$ states $(H=$ heavy, $L=$ light):

$$
\Delta m_{d}=\frac{G_{F}^{2}}{6 \pi^{2}} m_{W}^{2} \eta_{c} S\left(x_{t}\right) A^{2} \lambda^{6}\left[(1-\bar{\rho})^{2}+\bar{\eta}^{2}\right] m_{B_{d}} f_{B_{d}}^{2} \hat{B}_{B_{d}},
$$

where $S\left(x_{t}\right)$ is a Inami-Lim function [7] and $x_{t}=m_{t}^{2} / M_{W}^{2}$ (with $m_{t}$ being the $t$ quark mass in the regularization scheme $\overline{M S}, m_{t}^{\overline{M S}}\left(m_{t}^{\overline{M S}}\right)$, and $m_{W}$ the $W$ boson mass). $\eta_{c}$ is the short distance correction now calculated at next-to-leading order in perturbative QCD. $f_{B_{d}}^{2} \hat{B}_{B_{d}}$ is a factor hiding all the non-perturbative effects that contribute to $\Delta m_{d}$. To be more precise, $f_{B_{d}}$ is the decay constant that we have introduced in section 1.1.1, and represents the probability for the quark and the anti quark to annihilate, and $\hat{B}_{B_{d}}$ is the bag factor which takes into account possible deviation from the vacuum saturation approximation. The factor $f_{B_{d}} \sqrt{\hat{B}_{B_{d}}}$ is provided by lattice QCD methods.

- The mixing frequency $\Delta m_{s}$ of $B_{s} \bar{B}_{s}$ oscillations. It is usually combined with $\Delta m_{d}$ through the ratio:

$$
\frac{\Delta m_{d}}{\Delta m_{s}}=\frac{m_{B_{d}} f_{B_{d}}^{2} \hat{B}_{B_{d}}}{m_{B_{s}} f_{B_{s}}^{2} \hat{B}_{B_{s}}}\left(\frac{\lambda}{1-\frac{\lambda^{2}}{2}}\right)^{2}\left[(1-\bar{\rho})^{2}+\bar{\eta}^{2}\right] .
$$

It gives a similar type of constraint as $\Delta m_{d}$ on the apex position of the triangle and has the advantage to minimize theoretical errors as the ratio $\xi=f_{B_{s}} \sqrt{\hat{B}_{B_{s}}} / f_{B_{d}} \sqrt{\hat{B}_{B_{d}}}$ is expected to be better determined than the individual terms entering in its expression. 
- Indirect $C P$ violation in the $K^{0} \bar{K}^{0}$ system, expressed in terms of the $\epsilon_{K}$ parameter :

$$
\epsilon_{K}=C_{\varepsilon} A^{2} \lambda^{6} \bar{\eta}\left[-\eta_{1} S\left(x_{c}\right)+\eta_{2} S\left(x_{t}\right)\left(A^{2} \lambda^{4}(1-\bar{\rho})\right)+\eta_{3} S\left(x_{c}, x_{t}\right)\right] \hat{B}_{K}
$$

where

$$
C_{\varepsilon}=\frac{G_{F}^{2} f_{K}^{2} m_{K} m_{W}^{2}}{6 \sqrt{2} \pi^{2} \Delta m_{K}} .
$$

$S\left(x_{i}\right)$ and $S\left(x_{i}, x_{j}\right)$ again are the Inami-Lim functions [7], being $x_{q}=m_{q}^{2} / m_{W}^{2}$ and including NLO corrections $[8,9,10], f_{K}$ is the kaon decay constant and $\Delta m_{K}$ the neutral kaon system mixing frequency. Here also, the parameter $B_{K}$ has to be predicted by lattice QCD.

The measurements of these quantities can be combined through a global fit to evaluate the parameters $\bar{\rho}$ and $\bar{\eta}$, taking into account the different experimental and theoretical uncertainties. This is done by two analyses, [11] and [12], which differ mainly by the statistical treatment of theoretical errors. Figure 1.3 shows the results of [11] in the $(\bar{\rho}, \bar{\eta})$ plane using only indirect measurements.

This determination can be compared with the one given by the direct measurements of the angles $\beta, \alpha, \sin (2 \beta+\gamma)$ and $\gamma$ (Figure1.4).

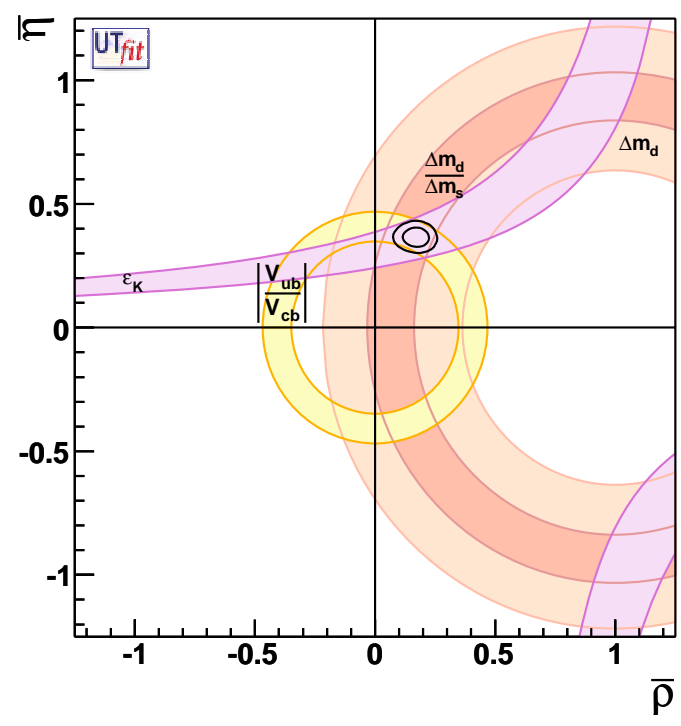

Figure 1.3: Allowed regions for $(\bar{\rho}, \bar{\eta})$ given by the measurements of $\left|V_{u b}\right| /\left|V_{c b}\right|, \epsilon_{K}, \Delta m_{d}$, and $\frac{\Delta m_{d}}{\Delta m_{s}}$. The closed contours at $68 \%$ and $95 \%$ probability are shown. The full lines correspond to $95 \%$ probability regions for each constraint. 


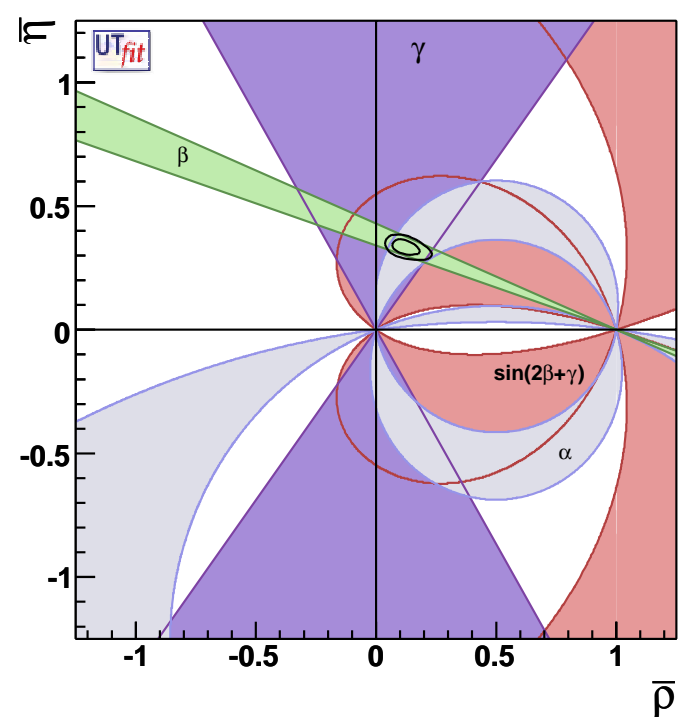

Figure 1.4: Allowed regions for $(\bar{\rho}, \bar{\eta})$ given by the direct measurements of the angles.

Given the accuracy achieved nowadays on all these measurements and on theoretical parameters, the two regions are compatible. The Standard Model seems to be very powerful in describing interactions between fermions and $C P$ violation. But we know that the Standard Model is incomplete and violations of its predictions are expected. The Standard Model is successful with a test at $10-20 \%$ accuracy. The precision need to be improved and this is possible thanks to the increase of the statistics that can lead to better measurements, on the angle $\gamma$ for example which is still largely limited by the statistics. We can also reach a better precision on the unitarity triangle parameters through a better knowledge of the effects of the strong interaction, that are parameterized in terms of form factors, and in the constants $\xi, f_{B_{d}} \sqrt{\hat{B}_{B_{d}}}$ and $B_{K}$. Since the amplitude of a semileptonic decay is proportional to the product of a CKM element by the form factors parameterizing the hadronic current (see Eq. (1.6)), charm semiletonic decays appear to be of particular interest in the understanding of the form factors as the CKM matrix elements involved are well known. If the form factors are well determined in the charm sector, they can constrain theoretical calculations. Once these calculations are validated, they can be used in the $B$ sector to extract the values of the CKM parameters $V_{u b}$ and $V_{c b}$. 


\subsection{Dynamics of Semileptonic Decays}

In this section we give qualitative arguments to understand the dynamics of semileptonic decays. After considerations that are common to all semileptonic decays, we focus on the decay of a pseudoscalar meson into a vector particle and we explain the decay rate of the $D_{s}^{+} \rightarrow \phi e^{+} \nu_{e}$ channel. In Section 1.2.3, we detail the general 5-dimensionnal dependence of the decay rate for the $D_{s}^{+} \rightarrow K^{+} K^{-} e^{+} \nu_{e}$ channel, including an $S$-wave component. As in this thesis we restrict the analysis to a narrow range centered on the $\phi$ mass peak, we only use the 4-dimensionnal decay rate, with no mass dependence. The way to obtain this mass integrated decay rate including the $S$-wave is explained in Section 1.2.4.

\subsubsection{The $q^{2}$ dependence}

The form factors, which parameterize the hadronic current introduced in Eq. (1.6), are function of the virtual $W$ mass squared, $q^{2}$. For a meson $M$ decaying into $X l^{-} \bar{\nu}$, we have :

$$
q^{2}=M_{W}^{2}=\left(p_{l}+p_{\nu}\right)^{2}=\left(P-p_{X}\right)^{2}=M^{2}+m_{X}^{2}-2 M E_{X}
$$

where $P$ and $p_{X}$ are the four momenta of the initial and final mesons and $E_{X}$ is the energy of $X$ in the $M$-meson rest frame. From this equation, we see that $q^{2}$ has a maximum value when the energy of $X$ is minimum $E_{X}=m_{X}$, which corresponds to $q_{\max }^{2}=\left(M-m_{X}\right)^{2}$. In this case, the virtual $W$ is as heavy as it can be, and both the $W$ and the daughter meson $X$ are produced at rest in the $M$ rest frame. Consequently, the lepton and neutrino, decay products of the $W$, are emitted back to back. The $q^{2}$ minimum value is $q_{\min }^{2}=m_{l}^{2} \sim 0$. In this case the lepton and the neutrino momenta are parallel. These different kinematic configurations are illustrated in Figure 1.5, for the decay of a $D_{s}^{+}$meson. Figure 1.5-a shows the initial meson, which contains a $c$-quark, and a spectator quark, $\bar{s}$. The $c$ quark decays into $c \rightarrow s e^{+} \nu$. When $q^{2}=q_{\max }^{2}$ (Figure 1.5-b), the daughter quark receives a little or no momentum kick, and the motion of the daughter quark relative to the spectator quark and the gluons (called the light degrees of freedom) is very similar to what it was before the decay. The formation of the daughter meson is thus favoured in this configuration. When $q^{2}=q_{\min }^{2}$ (Figure 1.5-c), the daughter quark $s$ recoiling against the $W$, receives a large kick, and moves rapidly with respect to the spectator quark. The hadronic system is thus more disturbed and the formation of a bound state is more difficult.

From these simple considerations one can observe that, for the form factors which depend on the overlap between the wave functions of the mesons in the initial and final states, the configurations at high $q^{2}$ (maximum overlap) are favoured in comparison with those at low 
$q^{2}$. Form factors are expected to be rising with the value of $q^{2}$.

Nevertheless, the influence of the spin of the daughter meson and of the available phasespace have to be taken into account when computing the differential decay rate versus $q^{2}$. For example, in the decay $D \rightarrow X W$, where $X$ is a pseudoscalar, as the $W$ has spin 1, the angular momentum balance implies that the decay occurs in a $P$-wave. As a result, the decay rate is proportional to $\left|p_{X}\right|^{3}$, and the high $q^{2}$ configuration is highly suppressed $\left(\left|p_{X}\right|\right.$ is the momentum of $X$ in the $W$ rest frame, $\left|p_{X}\right|=0$ at $q^{2}=q_{\text {max }}^{2}$ ). More generally, the decay rate is zero in this configuration because of phase space limitation.

a)
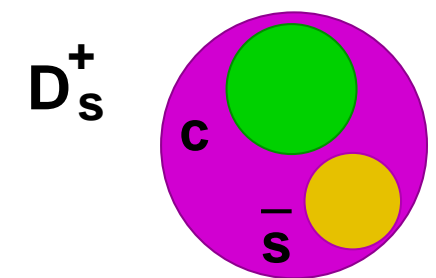

b)

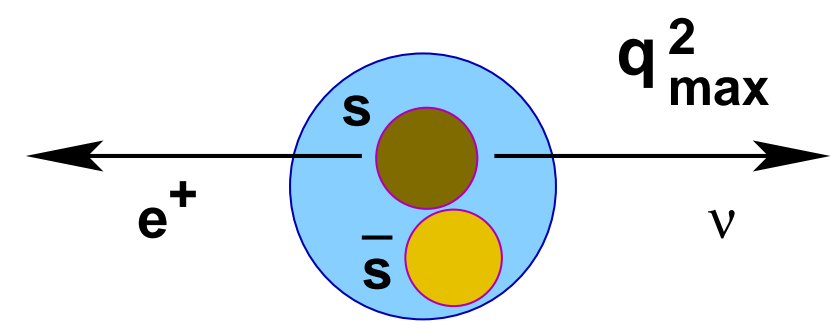

c)

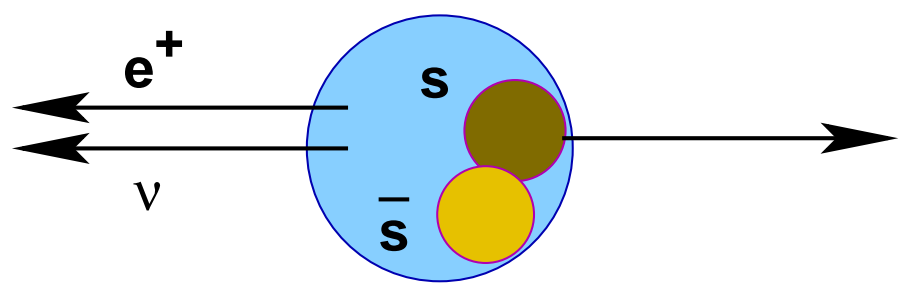

$\mathbf{9}_{\min }^{2}$

Figure 1.5: Kinematic configurations for the $D_{s}^{+} \rightarrow \phi e^{+} \nu_{e}$ decay in the $D_{s}^{+}$rest frame: (a) $D_{s}^{+}$before decay, (b) decay configuration for $q^{2}=q_{\text {max }}^{2}$, (c) decay configuration for $q^{2}=q_{\min }^{2}$.

\subsection{2 $D_{s}^{+} \rightarrow \phi e^{+} \nu_{e}$ decay rate}

In a first time, we consider the semileptonic decay $P(Q \bar{q}) \rightarrow V\left(q^{\prime} \bar{q}\right) \ell \nu$, where $V$ is a vector particle decaying into two pseudoscalars $\left(V \rightarrow P_{1} P_{2}\right)$, and we restrict to the case of $\ell=e$, 
for which we can assume that the lepton is massless. The hadronic current is a Lorentz 4 -vector with negative (vector) or positive (axial-vector) parity components. From Lorentz invariance, this current must be constructed from the four-vectors which characterize the particles produced in the final state, namely their momenta and the spin polarization of the vector meson. Using the formalism developped in [13], which is coming from [14] and [15] , the hadronic current is written with the general form:

$$
\begin{aligned}
<V(p, \epsilon)\left|V^{\mu}-A^{\mu}\right| P(p)>= & \frac{2 i \epsilon^{\mu \nu \alpha \beta}}{M+m_{V}} \epsilon_{\nu}^{*} p_{\alpha}^{\prime} p_{\beta} V\left(q^{2}\right)-\left(M+m_{V}\right) \epsilon^{* \mu} A_{1}\left(q^{2}\right) \\
& +\frac{\epsilon^{*} \cdot q}{M+m_{V}}\left(p+p^{\prime}\right)^{\mu} A_{2}\left(q^{2}\right)
\end{aligned}
$$

$\epsilon$ is the polarization of the vector meson, $V^{\mu}=\bar{q}^{\prime} \gamma^{\mu} Q$ and $A^{\mu}=\bar{q}^{\prime} \gamma^{\mu} \gamma_{5} Q$ are the vector and axial-vector currents. The dimensionless form factors $A_{1}\left(q^{2}\right)$ and $A_{2}\left(q^{2}\right)$ can be associated to the exchange of hadronic states with quantum number $J^{P}=1^{+}$, and $V\left(q^{2}\right)$ to states with $J^{P}=1^{-}$.

In the following, we consider the decay $D_{s}^{+} \rightarrow \phi e^{+} \nu_{e}$, with $\phi \rightarrow K^{+} K^{-}$as it is the main subject of this thesis, but the formalism can be applied to any $P(Q \bar{q}) \rightarrow V\left(q^{\prime} \bar{q}\right) \ell \nu, V \rightarrow P_{1} P_{2}$ decays ${ }^{1}$. The decay diagram for $D_{s}^{+} \rightarrow \phi e^{+} \nu_{e}$ is given in Figure 1.6.

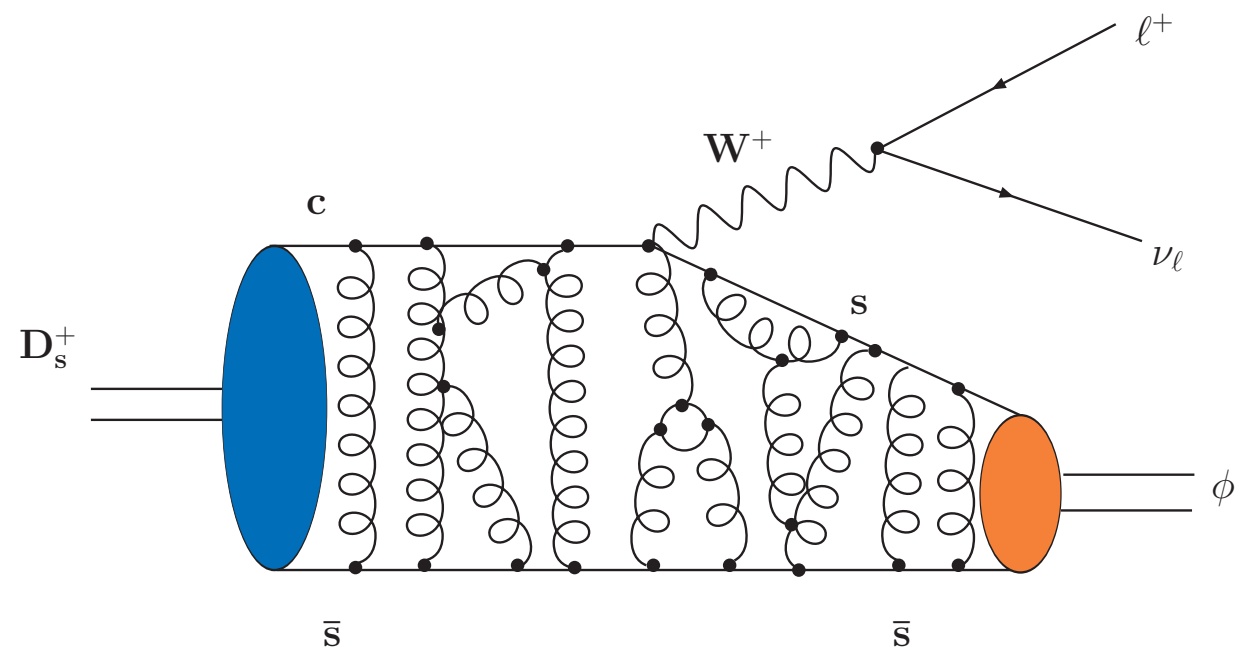

Figure 1.6: Illustrative diagram for the decay $D_{s}^{+} \rightarrow \phi e^{+} \nu_{e}$. Strong interaction effects between quarks are indicated by gluon exchanges.

\footnotetext{
${ }^{1}$ Because of helicity considerations, some signs have to be changed in the decay rate if we consider a $B$-meson instead of a charm meson, as the $W^{-}$has opposite helicity compared with the $W^{+}$. A general expression can be found in [13].
} 
The decay rate for $D_{s}^{+} \rightarrow K^{+} K^{-} e^{+} \nu_{e}$ depends on five variables (this is a general property of final states with four particles) whose definitions are illustrated on Figure 1.7:

- $m^{2}$ the mass squared of the $K^{+} K^{-}$system;

- $q^{2}$ : the mass squared of the $e^{+} \nu_{e}$ system;

- $\cos \left(\theta_{e}\right)$, where $\theta_{e}$ is the angle formed by the charged lepton three-momentum in the $e \nu$ rest frame and the line of flight of the $e \nu$ in the $D_{s}^{+}$rest frame;

- $\cos \left(\theta_{V}\right)$, where $\theta_{V}$ is the angle formed by the $K^{+}$three-momentum in the $K^{+} K^{-}$rest frame and the line of flight of the $K^{+} K^{-}$in the $D_{s}^{+}$rest frame;

- $\chi$, the angle between the normals to the planes defined in the $D_{s}^{+}$rest frame by the $K^{+} K^{-}$pair and the $e \nu$ pair. The sense of the angle is from the normal to the $K^{+} K^{-}$ plane to the normal to the $e \nu$ plane. $\chi$ is defined between $-\pi$ and $+\pi$.

If the decay of a $D_{s}^{-}$is measured, instead of a $D_{s}^{+}$, the direction of the $K^{-}$must be used to define $\cos \left(\theta_{V}\right)$ and $\chi$ is changed to $-\chi$. In this section, we neglect the dependence of the decay rate versus the vector meson mass, which is a doable assumption as the $\phi$ is narrow, but a more complete study will be done in section 1.2.

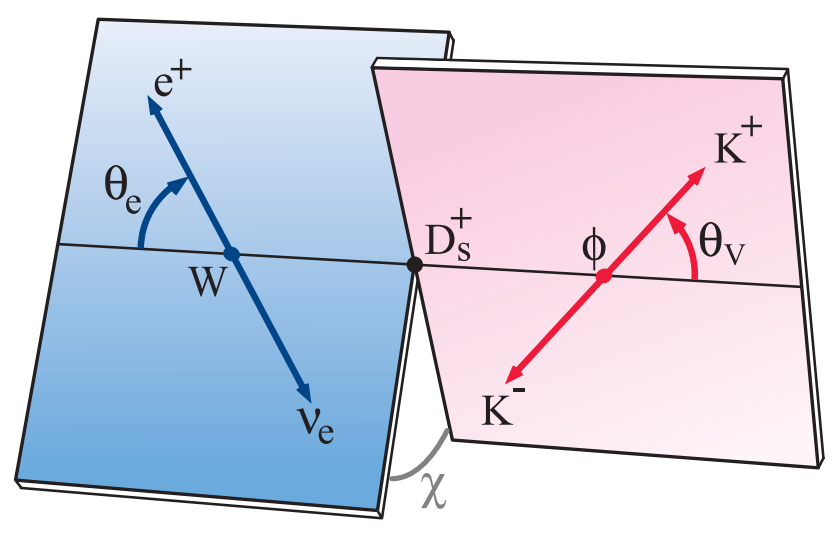

Figure 1.7: Definition of kinematic variables.

Once the angles are defined, then the decay rate dependence versus these variable is fixed and it can be written [13]:

$$
d^{4} \Gamma=\frac{3 G_{F}^{2}\left|V_{c s}\right|^{2}}{8(4 \pi)^{4} m_{D}^{3}} X q^{2} B R\left(V \rightarrow P_{1} P_{2}\right) \mathcal{I}_{R}\left(q^{2}, \theta_{V}, \theta_{e}, \chi\right) d q^{2} d \cos \left(\theta_{V}\right) d \cos \left(\theta_{e}\right) d \chi
$$


The dependence of $\mathcal{I}_{R}$ on $\theta_{e}, \theta_{V}$ and $\chi$ is given by:

$$
\begin{aligned}
\mathcal{I}_{R}= & \left(1+\cos \theta_{e}\right)^{2} \sin ^{2} \theta_{V}\left|H_{+}\left(q^{2}\right)\right|^{2} \\
& +\left(1-\cos \theta_{e}\right)^{2} \sin ^{2} \theta_{V}\left|H_{-}\left(q^{2}\right)\right|^{2} \\
& +4 \sin ^{2} \theta_{e} \cos ^{2} \theta_{V}\left|H_{0}\left(q^{2}\right)\right|^{2} \\
& +4 \sin \theta_{e}\left(1+\cos \theta_{e}\right) \sin \theta_{V} \cos \theta_{V} \cos \chi H_{+}\left(q^{2}\right) H_{0}\left(q^{2}\right) \\
& -4 \sin \theta_{e}\left(1-\cos \theta_{e}\right) \sin \theta_{V} \cos \theta_{V} \cos \chi H_{-}\left(q^{2}\right) H_{0}\left(q^{2}\right) \\
& -2 \sin ^{2} \theta_{e} \sin ^{2} \theta_{V} \cos 2 \chi H_{+}\left(q^{2}\right) H_{-}\left(q^{2}\right) .
\end{aligned}
$$

$H_{+,-, 0}\left(q^{2}\right)$ are the $\phi$ helicity amplitudes. The $0,+1,-1$ components are coming from the fact that, as the initial meson has spin zero, the polarization of the $W$ and of the vector meson have to be opposite (when evaluated in a common frame).

The decay rate variation versus the angles $\theta_{e}, \theta_{V}$ and $\chi$ can be understood from helicity and angular momentum balance considerations (see for instance [16]). As the $\nu_{e}$ is lefthanded and the $e^{+}$is right-handed (neglecting the electron mass), the helicity of the $e^{+} \nu_{e}$ system, measured along the the $e^{+}$direction, in the $W^{+}$rest frame, is equal to +1 . One can then express the $W^{+}$polarization in terms of its transverse and longitudinal components using as reference axis, the direction of the $W^{+}$in the $D_{s}^{+}$rest frame, as shown in Figure 1.8. Along this axis, the helicity of the $W^{+}$is $+1,-1$ or 0 with amplitudes which are proportional, respectively to the Wigner rotation matrices $d_{m, 1}^{1}\left(\cos \theta_{e}\right)$ namely: $\frac{1}{2}\left(1+\cos \theta_{e}\right)$, $\frac{1}{2}\left(1-\cos \theta_{e}\right)$ and $\frac{1}{\sqrt{2}} \sin \theta_{e}$.

We consider now the spin of the $\phi$ meson. To be combined with the $W^{+}$spin, its component have to be evaluated in projection along the $W^{+}$direction and in the same reference frame (the $W^{+}$rest frame). As the $D_{s}^{+}$meson is a pseudo-scalar, conservation of angular momentum implies that amplitudes of opposite helicities for the $\phi$ and the $W^{+}$are combined.

Since the decay products of the $\phi$ meson are two pseudo-scalar particles, the helicity of the $\phi$ measured along the direction defined by the kaons, in the $\phi$ rest frame, is zero. It is then necessary to rotate this initial reference axis by the angles $\theta_{V}$ and $\chi$ and to boost the components from the $\phi$ to the $W^{+}$rest frames. The helicity components of the $\phi$ measured along its flight direction, in the $D_{s}^{+}$rest frame are proportional to $d_{m, 0}^{1}\left(\cos \theta_{V}\right)$ namely: $\frac{1}{\sqrt{2}} \sin \theta_{V} e^{-i \chi},-\frac{1}{\sqrt{2}} \sin \theta_{V} e^{i \chi}$ and $\cos \theta_{V}$. These components are then boosted $\left(\gamma=E_{W} / q\right)$ to the $W^{+}$rest frame and only the third component is affected.

$$
\frac{E_{W}}{q}=\frac{M^{2}}{2 m_{V} q}\left(1-\frac{m_{V}^{2}}{M^{2}}-\frac{q^{2}}{M^{2}}\right)
$$




\begin{tabular}{|c|c|c|}
\hline helicity & $W+$ & $\phi$ \\
\hline$H_{+}\left(q^{2}\right)$ & $\frac{1}{2}\left(1+\cos \theta_{e}\right)$ & $\frac{1}{\sqrt{2}} \sin \theta_{V} e^{-i \chi}$ \\
\hline$H_{-}\left(q^{2}\right)$ & $\frac{1}{2}\left(1-\cos \theta_{e}\right)$ & $-\frac{1}{\sqrt{2}} \sin \theta_{V} e^{i \chi}$ \\
\hline$H_{0}\left(q^{2}\right)$ & $\frac{1}{\sqrt{2}} \sin \theta_{e}$ & $\cos \theta_{V}$ \\
\hline
\end{tabular}

Table 1.2: Summary of the different helicity amplitudes.

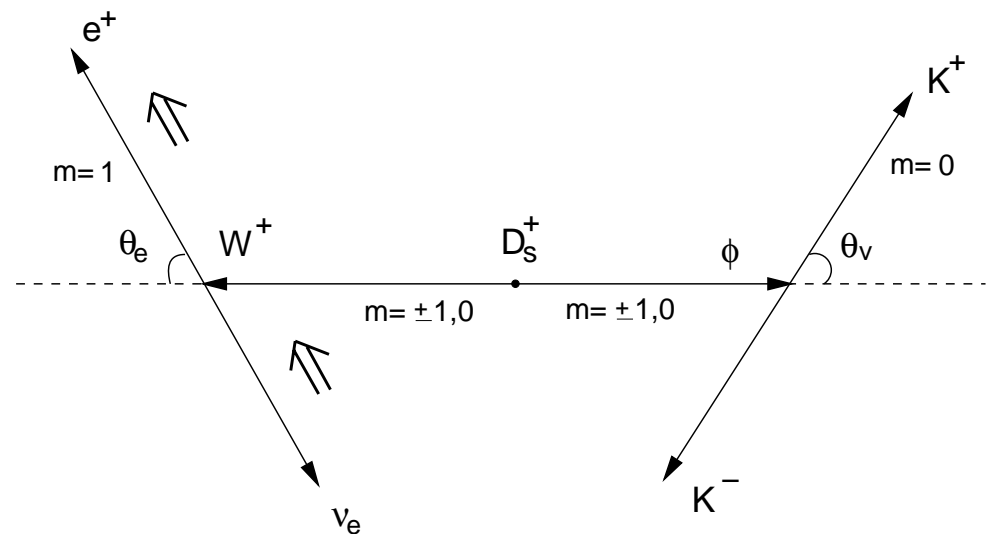

Figure 1.8: Representation of the helicity states of the particles involved in the decay $D_{s}^{+} \rightarrow$ $\phi e^{+} \nu_{e}$.

The hadronic current given in Eq. (1.24) can now be expressed in terms of the three helicity components. It can be noted that, as we have neglected the lepton mass it reduces to a three-vector. The helicity amplitudes $H_{+,-, 0}\left(q^{2}\right)$ are thus related to the two axial-vector form factors $A_{1,2}\left(q^{2}\right)$, and to the vector form factor $V\left(q^{2}\right)$ :

$$
\begin{aligned}
H_{0}\left(q^{2}\right) & =\frac{1}{2 m_{\phi} q}\left[\left(m_{D s}^{2}-m_{\phi}^{2}-q^{2}\right)\left(m_{D s}+m_{\phi}\right) A_{1}\left(q^{2}\right)-4 \frac{m_{D s}^{2} p_{\phi}^{2}}{m_{D s}+m_{\phi}} A_{2}\left(q^{2}\right)\right] \\
H_{ \pm}\left(q^{2}\right) & =\left(m_{D s}+m_{\phi}\right) A_{1}\left(q^{2}\right) \mp \frac{2 m_{D s} p_{\phi}}{m_{D s}+m_{\phi}} V\left(q^{2}\right) .
\end{aligned}
$$

The longitudinal component is multiplied by $1 / q$ as compared with the transverse components. This effect is related to the boost $\left(E_{W} / q\right)$ we have mentioned.

The decay amplitude $(\mathcal{A})$ is obtained by combining leptonic and hadronic amplitudes of opposite helicities. It is the sum of three contributions; each one corresponds to the product of the different terms present in each line of Table 1.2. The differential decay rate, given in Eq. (1.26), corresponds to $|\mathcal{A}|^{2}$.

This approach is quite general and applies to every hadronic final state decaying into two pseudoscalar mesons, independently of the total spin of this state. Only three hadronic 
form factors contribute, corresponding to the \pm 1 and 0 helicity values. If the hadronic state is a scalar or a pseudoscalar particle, only the helicity 0 is possible and the decay rate is proportionnal to $\left|H_{0}\left(q^{2}\right)\right|^{2}$.

There are peculiar cases when $q^{2}$ takes its extreme values. If $q^{2}=q_{m a x}^{2}$, both the $W$ and the vector meson are at rest. Their helicities are thus not defined and the $\cos \left(\theta_{\ell}\right)$ and $\cos \left(\theta_{V}\right)$ distributions become uniform. If $q^{2}=q_{m i n}^{2}$, the lepton and neutrino are parallel in the $D_{s}^{+}$ frame and their combined spin projection along their direction is zero. The spin projection of the $\phi$ should be zero also and we have only the terms proportional to $\left|d_{1,0}^{1}\left(\cos \theta_{\ell}\right)\right|^{2} \propto \sin ^{2} \theta_{\ell}$ for the $W$ and $\left|d_{0,0}^{1}\left(\cos \theta_{V}\right)\right|^{2} \propto \cos ^{2} \theta_{V}$ for the vector meson.

\subsubsection{The general $D_{s}^{+} \rightarrow K^{+} K^{-} e^{+} \nu_{e}$ decay rate}

In this thesis, we also investigate the $D_{s}^{+} \rightarrow K^{+} K^{-} e^{+} \nu_{e}$ decay, in order to find a possible $S$-wave component, through its interference with the $\phi$ meson. The presence of an $S$-wave component has been established in the decay $D^{+} \rightarrow K^{-} \pi^{+} \ell \nu$ by the FOCUS collaboration ([17],[18]) and the CLEO collaboration [19]. The FOCUS collaboration also put a limit on the presence of an $S$-wave contribution in the $D_{s}^{+} \rightarrow K^{+} K^{-} e^{+} \nu_{e}$ channel at " $5 \%$ of the maximum of the $\phi$ Breit-Wigner peak in the $H_{0}$ piece with a $90 \%$ confidence level" [20].

The existence of an $S$-wave in the $D_{s}^{+} \rightarrow K^{+} K^{-} e^{+} \nu_{e}$ channel corresponds to a $K^{+} K^{-}$ system in a $J^{P}=0^{+}$state. Because of the $c \rightarrow W^{+} s$ coupling and of the presence of an $\bar{s}$ spectator quark, the present study is sensitive only to the $s \bar{s}$ component of the $0^{+}$ states which can then be explored. The $f_{0}(980)$ is a possible candidate, as it has a mass of $980 \mathrm{MeV} / \mathrm{c}^{2}$ and a large width that allows the interference with the $\phi^{2}$. Theoretically, a $D$-wave component, corresponding to $J^{P}=2^{+}$could also be possible. But there is no evidence of such a state with a mass not too far from the $\phi$ resonance.

We use the formalism given in [21], which is similar to [22], and provides a partial wave decomposition for the hadronic system. Any dependence with the lepton mass has been neglected as electrons or positrons have been used.

$$
d^{5} \Gamma=\frac{G_{F}^{2}\left|V_{c s}\right|^{2}}{(4 \pi)^{6} m_{D}^{3}} X \beta \mathcal{I}\left(m^{2}, q^{2}, \theta_{V}, \theta_{e}, \chi\right) d m^{2} d q^{2} d \cos \left(\theta_{V}\right) d \cos \left(\theta_{e}\right) d \chi .
$$

In this expression, $X=p_{K K} * m_{D}$ where $p_{K K}$ is the momentum of the $K K$ system in the $D$ rest frame and $\beta=2 p^{*} / m$ where $p^{*}$ is the $K$ momentum in the $K K$ rest frame.

\footnotetext{
${ }^{2}$ the $\phi$ resonance peaks at $1019 \mathrm{MeV} / \mathrm{c}^{2}$, and has a width of $4.26 \mathrm{MeV} / \mathrm{c}^{2}$
} 
The dependence of $\mathcal{I}$ on $\theta_{e}$ and $\chi$ is given by:

$$
\begin{aligned}
\mathcal{I}= & \mathcal{I}_{1}+\mathcal{I}_{2} \cos 2 \theta_{e}+\mathcal{I}_{3} \sin ^{2} \theta_{e} \cos 2 \chi \\
& +\mathcal{I}_{4} \sin 2 \theta_{e} \cos \chi+\mathcal{I}_{5} \sin \theta_{e} \cos \chi \\
& +\mathcal{I}_{6} \cos \theta_{e}+\mathcal{I}_{7} \sin \theta_{e} \sin \chi \\
& +\mathcal{I}_{8} \sin 2 \theta_{e} \sin \chi+\mathcal{I}_{9} \sin ^{2} \theta_{e} \sin 2 \chi
\end{aligned}
$$

where $\mathcal{I}_{1, \ldots, 9}$ depend on $m^{2}, q^{2}$ and $\theta_{V}$. These quantities can be expressed in terms of three form factors, $\mathcal{F}_{1,2,3}$.

$$
\begin{aligned}
& \mathcal{I}_{1}=\frac{1}{4}\left\{\left|\mathcal{F}_{1}\right|^{2}+\frac{3}{2} \sin ^{2} \theta_{V}\left(\left|\mathcal{F}_{2}\right|^{2}+\left|\mathcal{F}_{3}\right|^{2}\right)\right\} \\
& \mathcal{I}_{2}=-\frac{1}{4}\left\{\left|\mathcal{F}_{1}\right|^{2}-\frac{1}{2} \sin ^{2} \theta_{V}\left(\left|\mathcal{F}_{2}\right|^{2}+\left|\mathcal{F}_{3}\right|^{2}\right)\right\} \\
& \mathcal{I}_{3}=-\frac{1}{4}\left\{\left|\mathcal{F}_{2}\right|^{2}-\left|\mathcal{F}_{3}\right|^{2}\right\} \sin ^{2} \theta_{V} \\
& \mathcal{I}_{4}=\frac{1}{2} \operatorname{Real}\left(\mathcal{F}_{1}^{*} \mathcal{F}_{2}\right) \sin \theta_{V} \\
& \mathcal{I}_{5}=\operatorname{Real}\left(\mathcal{F}_{1}^{*} \mathcal{F}_{3}\right) \sin \theta_{V} \\
& \mathcal{I}_{6}=\operatorname{Real}\left(\mathcal{F}_{2}^{*} \mathcal{F}_{3}\right) \sin { }^{2} \theta_{V} \\
& \mathcal{I}_{7}=\operatorname{Im}\left(\mathcal{F}_{1} \mathcal{F}_{2}^{*}\right) \sin \theta_{V} \\
& \mathcal{I}_{8}=\frac{1}{2} \operatorname{Im}\left(\mathcal{F}_{1} \mathcal{F}_{3}^{*}\right) \sin \theta_{V} \\
& \mathcal{I}_{9}=-\frac{1}{2} \operatorname{Im}\left(\mathcal{F}_{2} \mathcal{F}_{3}^{*}\right) \sin ^{2} \theta_{V}
\end{aligned}
$$

We can then expand the form factors $\mathcal{F}_{1,2,3}$ into partial waves to have their explicit dependence on $\theta_{V}$. If we restrict this expansion to $\mathrm{S}$ and $\mathrm{P}$ waves one obtains:

$$
\begin{aligned}
\mathcal{F}_{1} & =\mathcal{F}_{10}+\mathcal{F}_{11} \cos \theta_{V} \\
\mathcal{F}_{2} & =\frac{1}{\sqrt{2}} \mathcal{F}_{21} \\
\mathcal{F}_{3} & =\frac{1}{\sqrt{2}} \mathcal{F}_{31}
\end{aligned}
$$

Form factors $\mathcal{F}_{i j}$ depend only on $m^{2}$ and $q^{2}$. $\mathcal{F}_{10}$ characterizes the S-wave contribution whereas $\mathcal{F}_{i 1}$ correspond to the contribution from the P-wave (the $\phi$ ). Thus, in Eq. (1.31), the terms contributing only to the $S$-wave are the ones proportional to $\left|\mathcal{F}_{10}\right|^{2}\left(\mathcal{I}_{1}\right.$ and 
$\mathcal{I}_{2}$ ), and the terms corresponding to the interference between the $\mathrm{S}$ and the $\mathrm{P}$ wave are proportional to $\mathcal{F}_{10} \mathcal{F}_{i 1}\left(\mathcal{I}_{1}, \mathcal{I}_{2}, \mathcal{I}_{4}, \mathcal{I}_{5}, \mathcal{I}_{7}\right.$ and $\left.\mathcal{I}_{8}\right)$.

Integrating Eq. (1.29) over $d m^{2}$, assuming that the $\phi$ meson is the only contribution $\left(\mathcal{F}_{10}=0\right)$, one gets the correspondance between $\mathcal{F}_{i 1}, i=1,2,3$ and the helicity form factors,

$$
\begin{aligned}
& \mathcal{F}_{11}=\alpha 2 \sqrt{2} q H_{0} A_{\phi}(m) \\
& \mathcal{F}_{21}=\alpha 2 q\left(H_{+}+H_{-}\right) A_{\phi}(m) \\
& \mathcal{F}_{31}=\alpha 2 q\left(H_{+}-H_{-}\right) A_{\phi}(m)
\end{aligned}
$$

The value of the normalization factor $\alpha$ depends on the way the Breit-Wigner amplitude $\left(A_{\phi}(m)\right)$ for the $\phi$ meson has been parameterized. In these expressions it has been assumed that the mass dependence can be factorized.

\section{P-wave parameterization as a function of $m$ and $q^{2}$}

For the $q^{2}$ dependence of the Lorentz invariant form factors, different parameterizations are

possible. They are developped in Section 1.3. For the time being, we consider a pole or a modified pole parameterization:

$$
\begin{aligned}
V\left(q^{2}\right) & =\frac{V(0)}{\left(1-\frac{q^{2}}{m_{D_{s}^{*}}^{2}}\right)\left(1-\alpha \frac{q^{2}}{m_{D_{s}^{*}}^{2}}\right)} \\
A_{1}\left(q^{2}\right) & =\frac{A_{1}(0)}{1-\frac{q^{2}}{m_{1}^{2}}} \\
A_{2}\left(q^{2}\right) & =\frac{A_{2}(0)}{1-\frac{q^{2}}{m_{1}^{2}}}
\end{aligned}
$$

where $m_{D_{s}^{*}}=2.112 \mathrm{GeV} / \mathrm{c}^{2}$ is the $D_{s}^{*}$ mass and $m_{1}$ is expected to be close to the mass of $D_{s 1}$ states $\left(2.459\right.$ and $\left.2.535 \mathrm{GeV} / \mathrm{c}^{2}\right)$.

For the mass dependence of the $\phi$ decaying into $K^{+} K^{-}$, we use a Breit-Wigner distribution,

$$
A_{\phi}(m)=\frac{F_{1}(m) \sqrt{m_{\phi} \Gamma_{\phi}^{0} \frac{m_{\phi}}{p_{0}^{*}}}}{m_{\phi}^{2}-m^{2}-i m_{\phi} \Gamma_{\phi}(m)} .
$$

This amplitude is normalized such that, for a narrow resonance with a pole which is far from 
the boundaries, the following integral holds ${ }^{3}$

$$
\int \frac{p^{*}}{m}\left|A_{\phi}(m)\right|^{2} d m^{2}=\pi
$$

In this expression:

- $m$ is the $K^{+} K^{-}$mass;

- $m_{\phi}$ is the $\phi$ pole mass;

- $\Gamma_{\phi}^{0}$ is the nominal width of the $\phi$ for $m=m_{\phi}, \Gamma_{\phi}^{0}=(4.26 \pm 0.05) \mathrm{MeV} / \mathrm{c}^{2}$;

- $\Gamma_{\phi}(m)$ is the mass dependent $\phi$ width;

- $F_{1}(m)=\frac{p^{*}}{p_{0}^{*}} \frac{B\left(p^{*}\right)}{B\left(p_{0}^{*}\right)}$ where $B$ is the Blatt-Weisskopf damping factor: $B=1 / \sqrt{1+r_{b}^{2} p^{* 2}}$. $p^{*}$ and $p_{0}^{*}$ are evaluated at the mass $m$ and $m_{\phi}$ respectively and depend also on the masses of the $\phi$ decay products. We use $r_{b}=3(\mathrm{GeV} / \mathrm{c})^{-1}$.

In the denominator the width $\Gamma_{\phi}(m)$ is the total width of the resonance, whereas in the numerator the mass dependence is relative to the studied decay channel. Thus, to be more precise:

$$
\begin{aligned}
\Gamma_{\phi}(m)= & \Gamma_{\phi}^{0}\left[0.49 \times \frac{p^{*}}{p_{0}^{*}} \frac{m_{\phi}}{m} F_{1}^{2}(m)\right. \\
& \left.+0.34 \times\left(\text { now for } K_{L}^{0} K_{S}^{0}\right)+0.17 \times(\text { now for } 3 \pi)\right]
\end{aligned}
$$

where the first contribution corresponds to the decay $\phi \rightarrow K^{+} K^{-}$, the second one to $\phi \rightarrow$ $K_{L}^{0} K_{S}^{0}$, and the last one to the $\phi$ decay into three pions.

\section{$S$-wave parameterization for $\mathcal{F}_{10}$}

In a similar way as for the P-wave, we introduce the correspondance between $\mathcal{F}_{10}$ and the invariant form factor(s). For an $S$-wave, only the helicity $H_{0}$ form factor is contributing. So far, we have used:

$$
\mathcal{F}_{10}=r_{0} f_{10}\left(q^{2}\right) A_{f_{0}}(m)
$$

\footnotetext{
${ }^{3}$ we suppose that the $\phi$ is infinitely narrow
} 
with

$$
f_{10}=p_{K K} \frac{m_{D}}{1-\frac{q^{2}}{m_{S}^{2}}} .
$$

The parameter $r_{0}$ defines the normalization of the $S$-wave amplitude $\mathcal{F}_{10}$. From Eq. (1.39) and, using the parameterization of $A_{f_{0}}(m)$ defined in the following, $r_{0}$ is expressed in $\mathrm{GeV}^{-1}$. The $q^{2}$ variation of the form factor is determined by the contribution of $J^{P}=1^{+}, c \bar{s}$ states. The corresponding pole mass value $m_{S}$ is thus expected to be close to $2.5 \mathrm{GeV} / \mathrm{c}^{2}$. $\mathcal{F}_{10}$ is proportional to $p_{K K}$ so that the $S$-wave decay rate is proportional to $p_{K K}^{3}$ as expected from the $P$-wave between the $0^{+}$and the virtual $W$.

For the $K^{+} K^{-}$final state, the candidate for a $0^{+}$component is the $f_{0}(980)$. As the pole of this resonance is below the $K^{+} K^{-}$threshold, it decays mainly into $\pi \pi$. The decay amplitude is usually described using a Flatté expression:

$$
A_{f_{0}}(m)=\frac{m_{f_{0}} g_{\pi}}{m_{f_{0}}^{2}-m^{2}-i m_{f_{0}} \Gamma_{f_{0}}}
$$

with $\rho_{i}=\sqrt{1-\frac{4 m_{i}^{2}}{m^{2}}}=\frac{2 p^{*}}{m}$ and $\Gamma_{f_{0}}=g_{\pi} \rho_{\pi}+g_{K} \rho_{K}$. Note that $\rho_{i}$ becomes imaginary when $m<2 m_{i}$ which corresponds to the actual situation for $f_{0} \rightarrow K K$, below the $K K$ threshold. If one separates decays into $\pi^{+} \pi^{-}, \pi^{0} \pi^{0}, K^{+} K^{-}$and $K^{0} \bar{K}^{0}$ the expressions for $\rho_{i}$ become:

$$
\begin{aligned}
\rho_{\pi} & =\frac{2}{3} \sqrt{1-\frac{4 m_{\pi^{+}}^{2}}{m^{2}}}+\frac{1}{3} \sqrt{1-\frac{4 m_{\pi^{0}}^{2}}{m^{2}}} \\
\rho_{K} & =\frac{1}{2} \sqrt{1-\frac{4 m_{K^{+}}^{2}}{m^{2}}}+\frac{1}{2} \sqrt{1-\frac{4 m_{K^{0}}^{2}}{m^{2}}}
\end{aligned}
$$

For the values of $m$ between the threshold for $K^{+}$and $K^{0}$ production, the contribution from the partial decay width into $K^{0} \bar{K}^{0}$ is imaginary. The expression used in the numerator of Eq. (1.40) is simply a convention.

Compared with previous experiments, BES [23] has measured the values of the $f_{0}$ decay parameters with better precision:

$$
\begin{aligned}
& \qquad m_{f_{0}}=(965 \pm 8 \pm 6) \mathrm{MeV} / \mathrm{c}^{2}, \quad g_{\pi}=(165 \pm 10 \pm 15) \mathrm{MeV} / \mathrm{c}^{2}, \\
& \text { and } g_{K} / g_{\pi}=4.21 \pm 0.25 \pm 0.21 .
\end{aligned}
$$

These values are used in the following. 


\subsubsection{The 4-dimensionnal $D_{s}^{+} \rightarrow K^{+} K^{-} e^{+} \nu_{e}$ decay rate}

To include, in Eq. (1.25), the effect from an $S$-wave, we have to integrate the mass dependence which was explicit in Eq. (1.29). The interval of integration is restricted to a small region centered on the $\phi$ nominal mass $\left(m_{\phi} \pm \delta\right.$, with $\left.\delta=10 \mathrm{MeV} / \mathrm{c}^{2}\right)$.

In practice we transform Eq. (1.25), which corresponds to the P-wave contribution, by changing:

$$
q^{2} \mathcal{I}_{R} \rightarrow \mathcal{I}_{R}^{S+P}=q^{2} \mathcal{I}_{R}+\mathcal{I}_{R}^{S}+\mathcal{I}_{R}^{S P} .
$$

$q^{2} \mathcal{I}_{R}$ is the P-wave component as it appears in Eq. (1.25), $\mathcal{I}_{R}^{S}$ corresponds to the $S$-wave decay rate and $\mathcal{I}_{R}^{S P}$ corresponds to the interference between $\mathrm{S}$ and $\mathrm{P}$ waves. We normalize the two additionnal contributions relative to the $\phi$ within the considered mass interval.

The phase variation of the $f_{0}$ over this mass interval can be neglected and, as the pole of the $f_{0}$ is close to the lower edge of this mass interval, the phase of the $f_{0}$ amplitude $\left(\delta_{f_{0}}\right)$ is close to $\pi / 2$. Numerically one obtains:

$$
\cos \left(\delta_{f_{0}}\right) \sim-\frac{2 \Delta}{\Gamma_{f_{0}}}\left(1-\frac{2 \Delta^{2}}{\Gamma_{f_{0}}^{2}}\right), \sin \left(\delta_{f_{0}}\right) \sim 1-\frac{2 \Delta^{2}}{\Gamma_{f_{0}}^{2}}
$$

where $\Gamma_{f_{0}}=g_{\pi} \rho_{\pi}+g_{K} \rho_{K} \sim 0.32 \mathrm{GeV} / \mathrm{c}^{2}$ and $\Delta=m_{\phi}-m_{f_{0}}=(0.055 \pm 0.010) \mathrm{GeV} / \mathrm{c}^{2}$.

When the $K^{+} K^{-}$mass varies over the range $\left[m_{\phi}-10 \mathrm{MeV} / \mathrm{c}^{2}, \mathrm{~m}_{\phi}+10 \mathrm{MeV} / \mathrm{c}^{2}\right]$, the phase of the $f_{0}$ varies in the following intervals:

$$
\cos \left(\delta_{f_{0}}\right) \in[-0.40,-0.28], \sin \left(\delta_{f_{0}}\right) \in[0.92,0.96] .
$$

\section{P-wave normalisation}

As we consider a narrow mass window centered on the $\phi$, the variation of $p^{*}$ with the $K^{+} K^{-}$ mass can be neglected and Eq. (1.37) gives:

$$
\mathcal{N}_{\mathcal{P}}=\int_{m_{\phi}-\delta}^{m_{\phi}+\delta} \frac{p^{*}}{m}\left|A_{\phi}(m)\right|^{2} d m^{2}=2 \arctan \frac{2 \delta}{\Gamma_{\phi}^{0}},
$$

instead of the value $\pi$ which was obtained with no restriction on the integration domain. 


\section{$S$-wave decay rate}

Normalizing the $S$-wave contribution relatively to the P-wave, we have

$$
\mathcal{I}_{R}^{S}=\frac{1}{\mathcal{N}_{\mathcal{P}}} \frac{1}{2} \sin ^{2} \theta_{l} \int_{m_{\phi}-\delta}^{m_{\phi}+\delta}\left|\mathcal{F}_{10}\right|^{2} \frac{p^{*}}{m} d m^{2}
$$

where $\mathcal{F}_{10}$ is given in Eq. (1.39). This equation can be written using explicitly the $S$-wave normalization $\mathcal{N}_{\mathcal{S}}$ :

$$
\mathcal{I}_{R}^{S}=\frac{\mathcal{N}_{\mathcal{S}}}{\mathcal{N}_{\mathcal{P}}} \frac{1}{2} \sin ^{2} \theta_{l} r_{0}^{2} f_{10}^{2}
$$

Using Eq. (1.40), the $S$-wave normalization in the narrow mass window $\left[m_{\phi} \pm \delta\right]$ is:

$$
\begin{aligned}
\mathcal{N}_{\mathcal{S}} & =\int_{m_{\phi}-\delta}^{m_{\phi}+\delta} \frac{p^{*}}{m}\left|A_{f_{0}}(m)\right|^{2} d m^{2} \\
& =p_{0}^{*}\left(\frac{g_{\pi}}{\Gamma_{f_{0}}}\right)^{2} 4 \delta .
\end{aligned}
$$

\section{$\mathrm{S}-\mathrm{P}$ interference}

Taking into account the six terms of Eq. (1.26) that contribute to the S-P interference, and normalizing this contribution relatively to the P-wave, we obtain:

$$
\begin{aligned}
\mathcal{I}_{R}^{S P} & =\frac{1}{\mathcal{N}_{\mathcal{P}}} \int_{m_{\phi}-\delta}^{m_{\phi}+\delta}\left[\operatorname{Real}\left(\mathcal{F}_{10} \mathcal{F}^{*}{ }_{11}\right) \cos \theta_{V} \sin ^{2} \theta_{l}\right. \\
& +\frac{1}{\sqrt{2}} \operatorname{Real}\left(\mathcal{F}^{*}{ }_{10} \mathcal{F}_{21}\right) \sin \theta_{V} \sin \theta_{l} \cos \theta_{l} \cos \chi \\
& +\frac{1}{\sqrt{2}} \operatorname{Real}\left(\mathcal{F}^{*}{ }_{10} \mathcal{F}_{31}\right) \sin \theta_{V} \sin \theta_{l} \cos \chi \\
& +\frac{1}{\sqrt{2}} \operatorname{Im}\left(\mathcal{F}_{10} \mathcal{F}^{*}{ }_{21}\right) \sin \theta_{V} \sin \theta_{l} \sin \chi \\
& \left.+\frac{1}{\sqrt{2}} \operatorname{Im}\left(\mathcal{F}_{10} \mathcal{F}^{*}{ }_{31}\right) \sin \theta_{V} \sin \theta_{l} \cos \theta_{l} \sin \chi\right] \frac{p^{*}}{m} d m^{2}
\end{aligned}
$$


The first term generates an asymmetry as a function of $\cos \theta_{V}$. The other terms are proportional to:

$$
\operatorname{Real}\left(\mathcal{F}_{10}^{*} \mathcal{F}_{i 1}\right) \propto\left|A_{\phi}\right|\left|A_{f_{0}}\right| \cos \left(\delta_{\phi}-\delta_{f_{0}}\right)
$$

or to

$$
\operatorname{Im}\left(\mathcal{F}_{10} \mathcal{F}_{i 1}^{*}\right) \propto\left|A_{\phi}\right|\left|A_{f_{0}}\right| \sin \left(\delta_{f_{0}}-\delta_{\phi}\right) .
$$

When integrating over the considered mass interval, terms proportional to $\cos \left(\delta_{\phi}\right)$ gives a contribution which can be neglected as it is symmetric and of opposite sign below and above the pole of the $\phi$ meson, so we obtain:

$$
\begin{aligned}
\mathcal{I}_{\mathcal{R}}^{\mathcal{S P}} & =\frac{\mathcal{N}_{\mathcal{S P}}}{\mathcal{N}_{\mathcal{P}}} \sqrt{2} r_{0} f_{10} q\left[2 H_{0} \cos \theta_{V} \sin ^{2} \theta_{l}\right. \\
& +H_{+} \sin \theta_{V} \sin \theta_{l} \cos \chi\left(1+\cos \theta_{l}\right) \sin \left(\delta_{f_{0}}\right) \\
& -H_{-} \sin \theta_{V} \sin \theta_{l} \cos \chi\left(1-\cos \theta_{l}\right) \sin \left(\delta_{f_{0}}\right) \\
& -H_{+} \sin \theta_{V} \sin \theta_{l} \sin \chi\left(1+\cos \theta_{l}\right) \cos \left(\delta_{f_{0}}\right) \\
& \left.-H_{-} \sin \theta_{V} \sin \theta_{l} \sin \chi\left(1-\cos \theta_{l}\right) \cos \left(\delta_{f_{0}}\right)\right]
\end{aligned}
$$

The integral over the mass distribution for the five terms involves the same expression which amounts to:

$$
\begin{aligned}
\mathcal{N}_{\mathcal{S P}} & =\int_{m_{\phi}-\delta}^{m_{\phi}+\delta} \frac{p^{*}}{m}\left|A_{f_{0}}(m)\right|\left|A_{\phi}(m)\right| \sin \delta_{\phi}(m) d m^{2} \\
& =2 \sqrt{p_{0}^{*}} \frac{g_{\pi}}{\Gamma_{f_{0}}} \sqrt{\Gamma_{\phi}^{0}} \arctan \frac{2 \delta}{\Gamma_{\phi}^{0}}
\end{aligned}
$$

\section{Final expression}

Using the equations obtained previously for $\mathcal{N}_{\mathcal{P}}, \mathcal{N}_{\mathcal{S}}$ and $\mathcal{N}_{\mathcal{S P}}$, the following expressions are obtained and can be used in Eq. 1.43 to include effects from the $S$-wave component.

$$
\mathcal{I}_{R}^{S}=f_{10}^{2} \sin ^{2} \theta_{l} r_{0}^{2} p_{0}^{*}\left(\frac{g_{\pi}}{\Gamma_{f_{0}}}\right)^{2} \delta \frac{1}{\arctan \frac{2 \delta}{\Gamma_{\phi}^{0}}} .
$$


We introduce the following variables: $S=2 A_{S}^{2} \delta / \arctan \frac{2 \delta}{\Gamma_{\phi}^{0}}$ and $A_{S}=r_{0} \sqrt{p_{0}^{*}} \frac{g_{\pi}}{\Gamma_{f_{0}}}$, which leads to:

$$
\mathcal{I}_{R}^{S}=\frac{1}{2} S f_{10}^{2} \sin ^{2} \theta_{l}
$$

In the same way, for the S-P interference we obtain:

$$
\begin{aligned}
\mathcal{I}_{\mathcal{R}}^{\mathcal{S P}} & =\sqrt{2} A_{S} \sqrt{\Gamma_{\phi}^{0}} f_{10} q\left[2 H_{0} \cos \theta_{V} \sin ^{2} \theta_{l}\right. \\
& +H_{+} \sin \theta_{V} \sin \theta_{l} \cos \chi\left(1+\cos \theta_{l}\right) \sin \left(\delta_{f_{0}}\right) \\
& -H_{-} \sin \theta_{V} \sin \theta_{l} \cos \chi\left(1-\cos \theta_{l}\right) \sin \left(\delta_{f_{0}}\right) \\
& -H_{+} \sin \theta_{V} \sin \theta_{l} \sin \chi\left(1+\cos \theta_{l}\right) \cos \left(\delta_{f_{0}}\right) \\
& \left.-H_{-} \sin \theta_{V} \sin \theta_{l} \sin \chi\left(1-\cos \theta_{l}\right) \cos \left(\delta_{f_{0}}\right)\right]
\end{aligned}
$$

\subsection{Form Factors in heavy hadron semileptonic decays}

From general properties of strong interactions, form factors are analytic functions of a complex variable, which is equal to $q^{2}$ on the positive real axis. The same function can describe different reactions in which contribute hadronic states with $J^{P}=1^{\mp}$ respectively for vector and axial-vector contributions. Using dispersion relations, it is possible to evaluate the form factor at a given value of $q^{2}$ from a contour integral in the complex plane $\left(\mathcal{R} e q^{2}, \mathcal{I} m q^{2}\right)$. This integral can then be expressed in terms of the singularities present in this plane.

Let's consider the decay $D \rightarrow K^{-} e^{+} \nu_{e}$ as an example. A single form factor contributes in this process and it corresponds to the coupling of the $W$ to $c \bar{s}$ vector states. The physical region corresponds to real values of $q^{2}$ between 0 and $\left(m_{D}-m_{K}\right)^{2}$. Singularities, in the complex plane are located on the real axis and correspond to a pole at $q^{2}=m_{D_{s}^{*}}^{2}$ and a cut along the positive real axis, starting at $q^{2}=\left(m_{D}+m_{K}\right)^{2}$, corresponding to on-shell $D K$ interactions.

$$
V\left(q^{2}\right)=\frac{\operatorname{Res}(V)_{q^{2}=m_{D_{s}^{*}}^{2}}}{m_{D_{s}^{*}}^{2}-q^{2}}+\frac{1}{\pi} \int_{\left(m_{D}+m_{K}\right)^{2}}^{\infty} \frac{\operatorname{Im} V(t)}{t-q^{2}-i \epsilon} d t
$$

These singularities are illustrated in Figure 1.9. Both the cut and the pole are beyond the physical $q_{m a x}^{2}$ value and thus can never be realized. The $q^{2}$ variation of the form factor depends on the relative importance of contributions from the pole and the cut. These contributions depend also on the distance between $q_{\max }^{2}$ and the first singularity. 


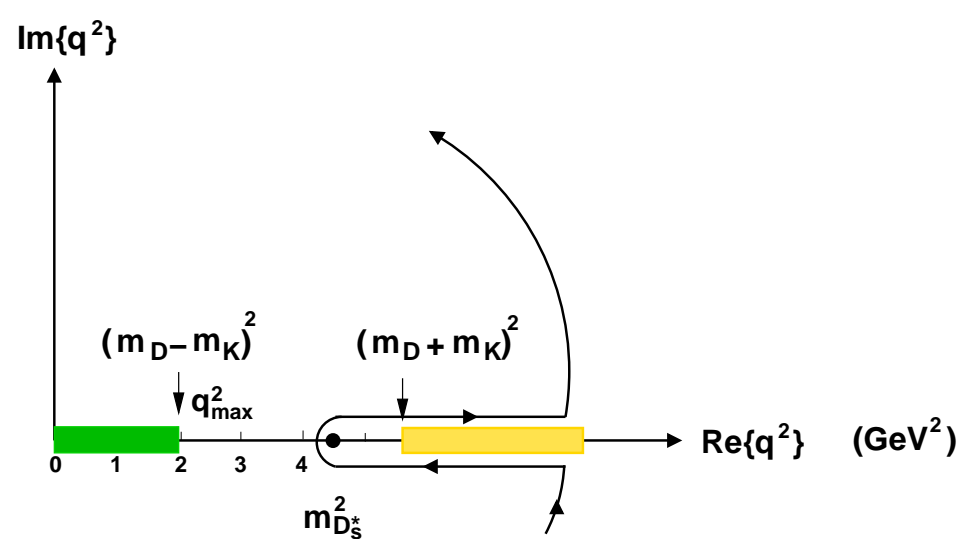

Figure 1.9: Representation of the contour used in the complex plane to write the relation dispersion of the vector form factor. The physical range correspond to $0<q^{2}<q_{\max }^{2}$. The singularities are the pole at the $D_{s}^{*}$ mass and the cut above $\left(m_{D}+m_{K}\right)^{2}$.

In the $D_{s}^{+} \rightarrow \phi e^{+} \nu_{e}$ decay, the situation is more complicated. For the axial vector current, the form factors $A_{1,2}$ have poles at the masses of the $1^{+}$states $D_{s 1}(2460)$ and $D_{s 1}(2536)$. For the vector form factor, the pole corresponds to the $D_{s}^{*}$. The opening of on-shell $D_{s} \phi$ events gives a cut starting at $8.9 \mathrm{GeV} / \mathrm{c}^{2}$. But, in between, there are several hadronic states which can contribute as, for instance, $D^{0} K^{+} \rightarrow D_{s} \phi$. Such states correspond also to cuts starting at the different thresholds. It can be noted that the physical region, between $q^{0}$ and $q_{\text {max }}^{2}=\left(m_{D_{s}^{+}}-m_{\phi}\right)^{2}=0.9 \mathrm{GeV}^{2}$, is rather distant from first singularities.

It is thus difficult to predict the $q^{2}$ dependence of the different form factors from this approach alone. Indeed general constraints have to be verified by hadronic form factors when considering peculiar situations which are explained in section 1.3.1. To determine the normalisation and the $q^{2}$ variation of the form factors one has to make use of lattice QCD calculations which incorporates all aspects of strong interactions. QCD sum rules and quark models provide also expectations which can be compared with present measurements. These methods are indicated in section 1.3.2. Finally, in section 1.3.3, we indicate the form factor parameterisations versus $q^{2}$ used in this analysis.

\subsubsection{Particular situations}

The normalisation and specific $q^{2}$ behaviour of the hadronic form factors can be obtained in some specified limits for which intermediate scales can be identified and allow an operator product expansion of the decay amplitude. 


\section{HQET}

When a heavy hadron decays semileptonically into another heavy hadron a new symmetry emerges which gives constraints on the behaviour of the form factors. This symmetry is exact in the limit of infinite quark mass values. For finite values of quark masses and depending on the form factors, these corrections start at order $1 / m_{Q}$ or $1 / m_{Q}^{2}$. These properties are used, at present, to determine the value of $V_{c b}$ from $B \rightarrow D^{*} e^{-} \nu$ decays (as corrections are expected at order $1 / m_{Q}^{2}$ in this case for $q^{2} \sim q_{\text {max }}^{2}$ ).

For charm, the value of the $c$-quark mass is of the order of 2-3 times the value of $\Lambda_{Q C D}$ but the strange quark cannot be considered as heavy. Thus, considerations from HQET are expected to receive large corrections when applied to charm semileptonic decays. In anycase we consider that it is of interest to review the constraints on the form factors, implied by HQET, and to indicate how they are violated in charm to eventually get insights for $B$ decays.

Instead of describing the form factors variation as function of $q^{2}$, HQET calculations use the quantity $w=v_{H} \cdot v_{P(V)}$, where $v_{H}$ and $v_{P(V)}$ are the four velocities of the initial and final hadrons. The variable $w$ is linearly related to $q^{2}$ by:

$$
w=\frac{m_{H}^{2}+m_{P(V)}^{2}-q^{2}}{2 m_{H} m_{P(V)}} .
$$

For $q^{2}=q_{\text {max }}^{2}, w=1$. HQET form factors contributing in $H \rightarrow P e \nu_{e}$ decays are usually labelled $h_{+}(w)$ and $h_{-}(w)$. For $H \rightarrow V e \nu_{e}$ decays they are: $h_{A 1}(w), h_{A 2}(w), h_{A 3}(w)$ and $h_{V}(w)$. In the limit of infinite quark masses, they verify:

$$
\begin{aligned}
h_{+}(w)=h_{V}(w)=h_{A 1}(w) & =h_{A 3}(w)=\xi(w), \\
h_{-}(w) & =h_{A 2}(w)=0,
\end{aligned}
$$

where $\xi(w)$ is the Isgur-Wise function [24]. It is normalized such that $\xi(1)=1$ which corresponds to a complete overlap of the mesons at $q_{\text {max }}^{2}$ but its variation with $w$ is not fixed by the theory.

The HQET form factors can be expressed in terms of the form factors $f_{+}, V, A_{1}, A_{2}$ introduced already [15]:

$$
f_{+}=\frac{m_{P}+m_{H}}{2 \sqrt{m_{P} m_{H}}}\left[h_{+}+\frac{m_{P}-m_{H}}{m_{P}+m_{H}} h_{-}\right]
$$




$$
\begin{aligned}
A_{1} & =\frac{\sqrt{m_{V} m_{H}}}{m_{V}+m_{H}}(1+w) h_{A_{1}} \\
A_{2} & =\frac{m_{V}+m_{H}}{2 \sqrt{m_{V} m_{H}}}\left(\frac{m_{V}}{m_{H}} h_{A_{2}}+h_{A_{3}}\right) \\
V & =\frac{m_{V}+m_{H}}{2 \sqrt{m_{V} m_{H}}} h_{V} .
\end{aligned}
$$

For infinite quark masses, these last equations can then be written:

$$
f_{+}\left(q^{2}\right)=V\left(q^{2}\right)=A_{2}\left(q^{2}\right)=\frac{A_{1}\left(q^{2}\right)}{1-\frac{q^{2}}{\left(m_{H}+m_{V}\right)^{2}}}=R^{-1} \xi\left(q^{2}\right)
$$

with $R=\frac{2 \sqrt{m_{H} m_{P(V)}}}{m_{H}+m_{P(V)}}$. It can be noted that in this limit one has also $m_{P}=m_{V}$. Equation (1.62) establish a relation between the form factor $f_{+}$, which contributes in $H \rightarrow P e \nu_{e}$ decays and the form factors $\left(V, A_{2}\right.$ and $\left.A_{1}\right)$ which parameterize $H \rightarrow V e \nu_{e}$ decays. This comes from another property of the heavy quark symmetry because of the decoupling of the heavy quark spin.

One can obtain also relations between the form factors having a universal character:

$$
\begin{aligned}
& r_{V}\left(q^{2}\right)=\frac{V\left(q^{2}\right)}{A_{1}\left(q^{2}\right)}=\frac{2}{w+1} \frac{1}{R^{2}} R_{1}(w) \\
& r_{2}\left(q^{2}\right)=\frac{A_{2}\left(q^{2}\right)}{A_{1}\left(q^{2}\right)}=\frac{2}{w+1} \frac{1}{R^{2}} R_{2}(w)
\end{aligned}
$$

with

$$
R_{1}(w)=\frac{h_{V}(w)}{h_{A 1}(w)}, R_{2}(w)=\frac{h_{A 3}+m_{V} / m_{H} h_{A 2}(w)}{h_{A 1}(w)}
$$

In the heavy quark symmetry limit: $R_{1}(1)=R_{2}(1)=1$ and $r_{V}\left(q_{\text {max }}^{2}\right)=r_{2}\left(q_{\max }^{2}\right)=R^{-2}$. It can be observed also that the ratios $r_{V}(0)$ and $r_{2}(0)$ are equal, respectively, to $R_{1}\left(w_{\max }\right)$ and $R_{2}\left(w_{\max }\right)$.

The $q^{2}$ range of validity of these expressions corresponds to situations in which the recoil energy of the light degrees of freedom in the parent rest frame are much smaller than the heavy quark mass: $(w-1) \ll m_{H} / \Lambda_{Q C D}$. If one compares numerically these situations, for $b \rightarrow c e \nu_{e}$ and $c \rightarrow s e \nu_{e}$, one gets:

$$
R_{b} \sim 0.88 \quad R_{c} \sim 0.81
$$




$$
(w-1)_{\text {max. }}^{b} \sim 0.6 \ll 25 \quad(w-1)_{\text {max. }}^{c} \sim 1 \ll 7.5
$$

So, numerically, the conditions of validity of the previous expressions seem also to be satisfied for charm semileptonic decays. Unfortunately the situation changes once corrections to the previous expressions are considered. These corrections correspond to an expansion in $1 / m$ where $m$ can take the values of the masses of the two quarks involved in the weak transition. Corrections comprise also an expansion in the strong coupling constant, from perturbative QCD. These corrections are explained in [15]. Some of the form factors are protected against $1 / \mathrm{m}$ corrections; in this case the expansion starts at order $1 / \mathrm{m}^{2}$.

Numerically, for $B \rightarrow D^{*} e \nu_{e}$, the following values have been quoted in [15]:

$$
\begin{aligned}
& R_{1}(w)=1.35-0.22(w-1)+0.09(w-1)^{2} \\
& R_{2}(w)=0.79+0.15(w-1)-0.04(w-1)^{2}
\end{aligned}
$$

More recently, BABAR obtained the following result [25], using a parameterization defined in [26]:

$$
\begin{aligned}
& R_{1}(w)=1.327-0.12(w-1)+0.05(w-1)^{2} \\
& R_{2}(w)=0.859+0.11(w-1)-0.06(w-1)^{2}
\end{aligned}
$$

It is an unambiguous prediction of HQET that $R_{1}(w)>1$ as both the QCD and $1 / m$ corrections are positive. For $R_{2}(w)$, QCD corrections are very small, and $1 / m$ corrections seem to decrease the value of $R_{2}(w)$. These considerations can be extended qualitatively to charm even if it is not possible simply to change $m_{c}$ into $m_{s}$ in the previous expressions. In [15], Neubert argues that we can believe in a "continuity of signs", leading to predictions for the $D \rightarrow V e \nu$ decays for which the tendency $R_{1}>1$ and $R_{2}<1$ should persist.

This approach has been investigated quantitatively in [27]. They compare measurements available at that time on $D \rightarrow \bar{K} / \bar{K}^{*} e^{+} \nu_{e}$ decays to evaluate the importance of perturbative QCD and $1 / m$ corrections. Their main conclusions were the following:

- first order perturbative QCD corrections are governed by $\alpha_{S}(\mu) / \pi \simeq 0.33$, where $\mu$ is a scale intermediate between the $c$ and $s$ quark masses;

- these corrections have a tendency to decrease the expected rates;

- QCD corrections alone cannot explain the measured ratio for $K^{*} / K \simeq 0.5$ production in $D$ semileptonic decays as they have rather similar effects on all form factors; 
- $1 / m$ corrections are needed to explain the measurements and have different contributions in the different form factors.

\section{LEET}

In the previous transitions the two short scales are given by the inverse of the two heavy quark masses. In the following limit one scale is still provided by the inverse of the mass of the decaying quark whereas the other corresponds to the inverse of the energy of the emitted hadron. As this energy is given, in the decaying hadron rest frame, by :

$$
E_{P(V)}=\frac{m_{H}}{2}\left[1-\frac{q^{2}}{m_{H}^{2}}+\frac{m_{P(V)}^{2}}{m_{H}^{2}}\right]
$$

it is maximal at $q^{2}=0$, so the new formalism is expected to be valid at low $q^{2}$ whereas HQET was valid close to $q_{\max }^{2}$. The Large Energy Effective Theory (LEET) has been originally introduced by Dugan and Grinstein [28]. In the following we have used the results obtained in [29]. The four invariant form factors appearing in semileptonic decays of $B$ and $D$ hadrons can be expressed in terms of three universal form factors: $\zeta(m, E), \zeta_{\perp}(m, E)$ and $\zeta_{\|}\left(m_{H}, E_{V}\right)$.

$$
\begin{aligned}
f_{+}\left(q^{2}\right) & =\zeta\left(m_{H}, E_{P}\right), \\
A_{1}\left(q^{2}\right) & =\frac{2 E_{V}}{m_{H}+m_{V}} \zeta_{\perp}\left(m_{H}, E_{V}\right), \\
A_{2}\left(q^{2}\right) & =\left(1+\frac{m_{V}}{m_{H}}\right)\left[\zeta_{\perp}\left(m_{H}, E_{V}\right)-\frac{m_{V}}{E_{V}} \zeta_{\|}\left(m_{H}, E_{V}\right)\right], \\
V\left(q^{2}\right) & =\left(1+\frac{m_{V}}{m_{H}}\right) \zeta_{\perp}\left(m_{H}, E_{V}\right) .
\end{aligned}
$$

It can be shown that these universal form factors can be expanded in $1 / m_{H}$ :

$$
\zeta(m, E)=\sqrt{m_{H}}\left[z^{(0)}(E)+\sum_{k=1}^{\infty} \frac{z^{(k)}(E)}{m_{H}^{k}}\right]
$$

and they thus satisfy (at first order) scaling relations:

$$
\zeta(m, E)=\sqrt{m_{H}} z(E)
$$

Using expressions given in (1.70) the ratio between the vector and axial form factors is 
obtained:

$$
r_{V}\left(q^{2}\right)=\frac{\left(m_{H}+m_{V}\right)^{2}}{m_{H}^{2}+m_{V}^{2}-q^{2}} .
$$

In particular this ratio, evaluated at $q^{2}=0$ is equal to 1.78 for $D \rightarrow \bar{K}^{*} e^{+} \nu_{e}$ and 1.82 for $D_{s} \rightarrow \phi e^{+} \nu_{e}$. For $r_{2}\left(q^{2}\right)$ the expression is more complicated and depends on the ratio between $\zeta_{\|}$and $\zeta_{\perp}$.

\section{Constraints from unitarity and analyticity}

It has been shown [30] that a general parameterization of form factors, entering in a semileptonic decay $H \rightarrow X e^{+} \nu_{e}$, which satisfies constraints from analyticity and unitarity reads:

$$
F(t)=\frac{1}{P(t) \Phi(t)} \sum_{k=0}^{\infty} a_{k} z\left(t ; t_{0}\right)^{k}
$$

where $t=q^{2}, \Phi(t)$ is a computable function arising from perturbative QCD and $P(t)$ depends only on the masses of mesons below the $H X$ pair production threshold that contribute as virtual intermediate states. The variable $z\left(t ; t_{0}\right)$ is a kinematic function of $t$ defined by:

$$
\frac{1+z\left(t ; t_{0}\right)}{1-z\left(t ; t_{0}\right)}=\sqrt{\frac{t_{+}-t}{t_{+}-t_{0}}}
$$

where $t_{+}$is the pair production threshold, in general $t_{+}=\left(m_{H}+m_{X}\right)^{2}$, and $t_{0}$ is a free parameter. The complex $t$ plane with its cut starting at $t_{+}$along the real axis is transformed into a disk in the $z$ complex plane, with a center that corresponds to $t_{0}$, of unit radius, and with a frontier corresponding to the cut. The coefficients $a_{k}$ are unknown constants constrained to obey:

$$
\sum_{k=0}^{\infty}\left(a_{k}\right)^{2} \leq 1
$$

The function $z\left(t ; t_{0}\right)$ takes its minimal physical value $z_{\text {min }}$ at $t=t_{-}=q_{\text {max }}^{2}=\left(m_{H}-m_{X}\right)^{2}$, vanishes at $t=t_{0}$ and reaches its maximum $z_{\max }$ at $t=q^{2}=0$. In the following we choose the value of $t_{0}$ which minimizes the value of $|z|$ :

$$
t_{0}=t_{+}\left(1-\sqrt{1-\frac{t_{-}}{t_{+}}}\right)
$$


In this case:

$$
z_{\text {max }}=-z_{\min }=\frac{t_{+}^{1 / 4}-\left(t_{+}-t_{-}\right)^{1 / 4}}{t_{+}^{1 / 4}+\left(t_{+}-t_{-}\right)^{1 / 4}} .
$$

This value is rather small for several semileptonic decay channels. The $z$ expansion can thus be limited to a linear expression. As an example it has been shown that this applies already for the decay $D^{0} \rightarrow K^{-} e^{+} \nu_{e}$ where $z_{\max } \simeq 0.05$. In addition, for $B$ semileptonic decays, Eq. (1.76) is effective to limit the values of additional terms in the expansion once the first coefficients are measured [26].

For the decay channels $B / D \rightarrow V e \nu_{e}$, expressions are provided in [31] for the functions $\Phi$ which enter in the expressions of the form factors $q H_{0}\left(q^{2}\right), V\left(q^{2}\right)$ and $A_{1}\left(q^{2}\right)$.

Poles at $t=t_{P_{i}}$, below $t_{+}$, correspond simply to the product:

$$
P(t)=\prod_{i} z\left(t ; t_{P_{i}}\right) .
$$

As we have explained already, for $D_{s}^{+} \rightarrow \phi e^{+} \nu_{e}$ the situation is not as simple as for $B / D \rightarrow$ $\pi e \nu_{e}$ or $D \rightarrow \bar{K} e \nu_{e}$. Between the poles and the opening of the $D_{s} \phi$ channel corresponding to $\left(m_{D_{s}}+m_{\phi}\right)^{2}$ several channels can also contribute. For the axial-vector form factors, the first threshold corresponds to $D^{*} K$ ocurring in a $\mathrm{S}$ wave process, so $t_{+}=\left(m_{D^{*}}+m_{K}\right)^{2}$. There is only one pole below this threshold, the $D_{s 1}(2459)$. For the vector form factor, the cut starts with the $D K$ channel (as for the $D^{0} \rightarrow K^{-} e^{+} \nu_{e}$ channel), thus $t_{+}=\left(m_{D}+m_{K}\right)^{2}$, and the pole below threshold is the $D_{s}^{*}$.

\subsubsection{Different theoretical approaches to determine hadronic form factors}

In the previous section we have indicated a few general contraints that apply to the behaviour of form factors. It remains to evaluate the normalisation and $q^{2}$ variation of these quantities. Several approaches have been tried which correspond to different approximations of QCD in the nonperturbative regime.

\section{Quark models}

Quark model calculations estimate meson wave functions and use them to compute the corresponding contribution that appears in the hadronic current. These integrals are evaluated 
at a particular value of $q^{2}, q^{2}=0$ or $q^{2}=q_{\max }^{2}$. The $q^{2}$ dependence is generally determined separately, using theoretical models. Among them, the ISGW model [32], and its update ISGW2 [33], are largely used. In particular, ISGW2 is the default in the BABAR simulation for most of semileptonic decays and in particular for the $D_{s}^{+} \rightarrow \phi e^{+} \nu_{e}$ channel. The distributions of the four kinematic variables for the $D_{s}^{+} \rightarrow \phi e^{+} \nu_{e}$ decay channel, using this model are given in Figure 1.10.
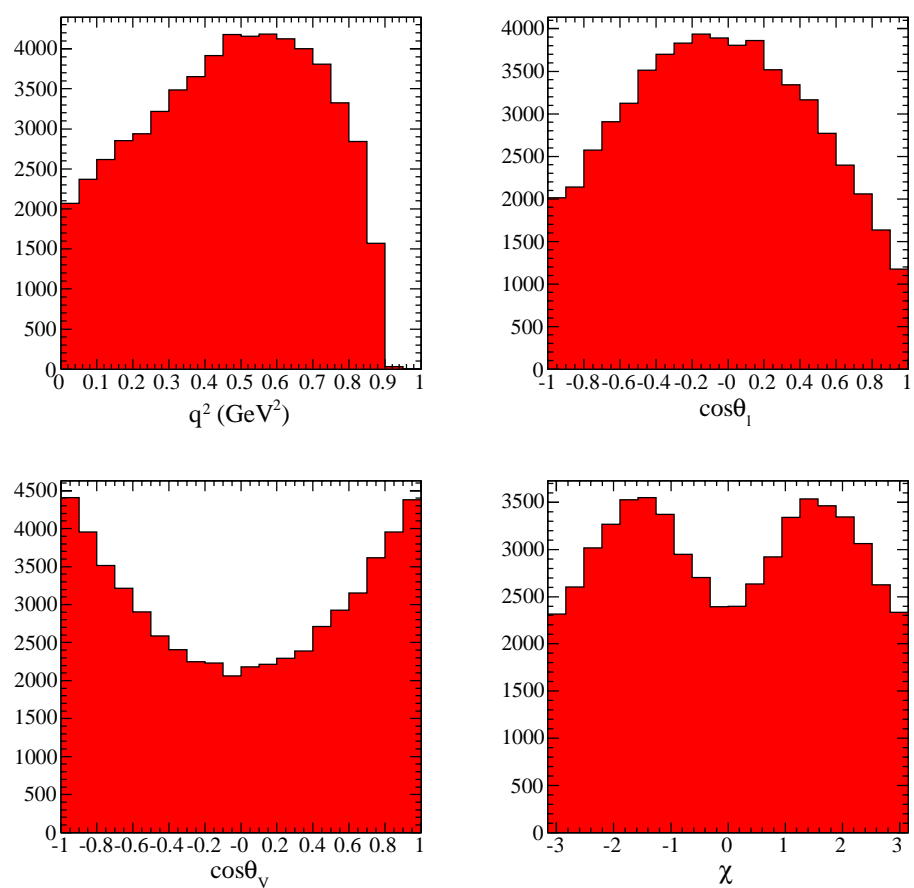

Figure 1.10: Distributions of the $D_{s}^{+} \rightarrow \phi e^{+} \nu_{e}$ differential decay rate versus $q^{2}, \cos \theta_{\ell}, \cos \theta_{V}$ and $\chi$, when the form factors are parameterized by the ISGW2 model. These plots are obtained using the BABAR default simulation.

Quark models incorporate now relativistic effects and, for the most recent versions, they satisfy the constraints from HQET and LEET, when applicable. Predictions from these models are usually dependent on the values of parameters as effective quark masses and slopes of meson wave functions. In Tables 1.3 we give a compilation of expectations from several models.

\section{QCD sum rule}

QCD sum rules [34], and their extension on the light cone, provide a non perturbative approach to form factor calculations. This method has the advantage to be based on the first principles of QCD, but it is expected to be valid only at low $q^{2}$. In [39], using QCD sum 


\begin{tabular}{|c|c||c|c|c|c|c|}
\hline Source & $f_{+}(0)$ & $A_{1}(0)$ & $A_{2}(0)$ & $V(0)$ & $r_{V}(0)$ & $r_{2}(0)$ \\
\hline BSW (1985)[14] & 0.76 & 0.88 & 1.2 & 1.3 & 1.48 & 1.36 \\
AW (1988)[35] & 0.7 & 0.8 & 0.6 & 1.5 & 1.87 & 0.75 \\
ISGW (1989)[32] & 0.82 & 0.8 & 0.8 & 1.1 & 1.37 & 1.0 \\
ISGW2 (1995)[33] & 0.76 & 0.88 & 1.15 & 1.27 & 1.44 & 1.30 \\
Jaus (1996)[36] & 0.78 & 0.66 & 0.43 & 1.04 & 1.58 & 0.65 \\
RQM (1996)[37] & $0.73 \pm 0.07$ & $0.63 \pm 0.06$ & $0.43 \pm 0.04$ & $0.62 \pm 0.06$ & 0.98 & 0.68 \\
MS (2000)[38] & 0.78 & 0.66 & 0.49 & 1.03 & 1.56 & 0.74 \\
\hline Expt.[4] & $0.73 \pm 0.01$ & & & & $1.62 \pm 0.08$ & $0.83 \pm 0.05$ \\
\hline \hline$B \rightarrow D / D^{*} e^{-} \bar{\nu}_{e}[25]$ & & & & & $1.37 \pm 0.08$ & $0.88 \pm 0.04$ \\
\hline
\end{tabular}

Table 1.3: Quark model expectations for the values of different form factor parameters entering in $D \rightarrow \bar{K} / \bar{K}^{*} e^{+} \nu_{e}$ decays. These values are compared with corresponding measurements and also with similar results obtained with B-mesons.

rules, they compute the ratios of form factors at $q^{2}=0$ for the $D_{s}^{+} \rightarrow \phi e^{+} \nu_{e}$ decay. They obtain $r_{2}=1.07 \pm 0.43$ and $r_{V}=2.20 \pm 0.85$. A calculation using light cone sum rules is done for $D_{s}^{+} \rightarrow \phi e^{+} \nu_{e}$ decay in [40]. They obtain: $r_{2}=1.31 \pm 0.43$ and $r_{V}=1.38 \pm 0.44$.

\section{Lattice QCD}

Lattice QCD, invented by Kenneth G. Wilson in 1974 [41] is a computation technique that aims to solve numerically the QCD equations, using a discretized euclidiean space-time. It is a non perturbative approach which uses the exact Lagrangian of QCD and the method of Feynman path integral. The value of an observable $\mathcal{O}$ is evaluated from the path integral:

$$
\mathcal{O}=\frac{1}{Z} \int \mathcal{D} A_{\mu} \mathcal{D} \psi \mathcal{D} \bar{\psi} \mathcal{O} e^{-S},
$$

with

$$
Z=\int \mathcal{D} A_{\mu} \mathcal{D} \psi \mathcal{D} \bar{\psi} e^{-S} .
$$

$S$ is the QCD action, $A_{\mu}$ are the gluons fields, $\psi$ and $\bar{\psi}$ represent the fermions fields. When discretizing the space-time, the fermion fields become located on the nodes of the lattice (also called the sites) and the gauge fields get bound to lattice links. The Yang-Mills action for gauge fields and the Dirac operator for fermions have to be transcribed on the lattice in such a way that they preserve fundamental properties of the theory as the gauge invariance and the chiral symmetry. The lattice is characterized by its spacing $a$ and its size $L$ as shown on Figure 1.11. Typical characteristics of actual lattices are $L \gtrsim 2 \mathrm{fm}$ and $a \lesssim 0.1 \mathrm{fm}$. 


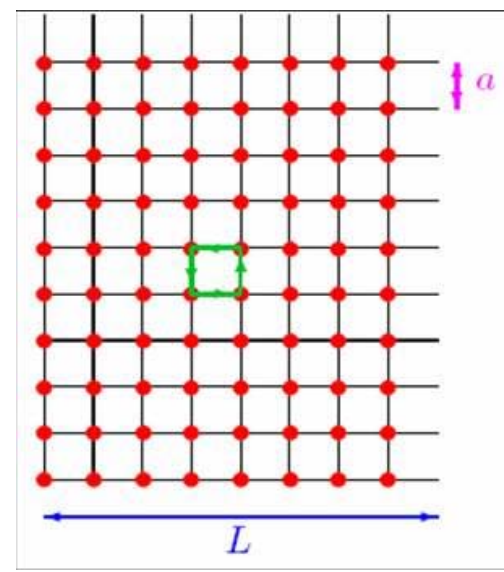

Figure 1.11: Representation of the lattice with size L and spacing a.

Even on a lattice of finite volume, the evaluation of the integral (1.80) cannot be done exactly as the range of the action $S$ is enormous. To solve this problem, the integral is calculated with Monte Carlo methods using large ensembles of gauge field configurations (several thousands), generated according to the law $e^{-S}$. The value of the observable $\mathcal{O}$ is then obtained by an average on the configurations. The first step of the lattice calculation consists in the generation of these configurations, which is the most time consuming operation. A method called Hybrid Monte Carlo (HMC) is used to improve the efficiency of the gauge configurations generation, but a large amount of matrix inversions due to the computation of the Dirac operator is still necessary. This propagator is a matrix with three indices: site, spin and color (this means 12 degrees of freedom per site). Each element of the matrix gives the amplitude for a quark on a given site to propagate to another space-time point. Operationally, the propagator calculation consists in an inversion of the Dirac operator which is a $12 N \times 12 N$ matrix, $N$ being the number of sites. A lot of elements of this matrix have zero value, and several techniques have been developped to make the compution of the inverse matrix faster but this operation still requires a large computional power.

Lattice QCD is very powerful because it uses directly the first principles of quantum field theory but the precision of the calculations is limited due to approximations that have to be made, often related to the computational cost. The following errors have to be taken into account:

- Statistical errors from the path integral evaluation. It is due to the finite number of configurations.

- Discretization errors, due to the size of lattice spacing a, and which arise when the derivatives in the action have to be approximated by finite differences. Typically, these 
errors are $O\left(a^{2}\right)$ but they depend on the discretized gauge and Dirac actions used. The effects of discretization can be corrected using several measurements performed with different lattice spacing, and extrapolating to $a=0$.

- Error due to the finite volume of the lattice, results have to be extrapolated to infinite volume.

- Chiral extrapolation: lattice computation becomes really costly and difficult for light quark masses, so the light quarks used on the lattice are heavier than the physical quarks (masses of $m_{u, d} \sim m_{s} / 10$ can be reached nowadays [42]). The extrapolation to the real quark masses can be done thanks to the chiral perturbation theory.

Until some years ago, all the calculations were performed in the "quenched" approximation, that is to say neglecting the quark loops, which was inducing large errors on the computed physical quantities (10 to $20 \%$ ). Nowadays, most of calculations take into account the sea-quark effects ("unquenched"), at least with two light quarks degenerated, or including also the strange quark. But here again, the light quark masses have to be extrapolated to their physical values.

Whereas the quarks up and down are difficult to simulate with their physical masses on the lattice, another problem arises for heavy quarks due to the discretization errors proportional to a positive power of $m_{Q} a$. Actually, discretization effects are small if the lattice spacing a is much smaller than the Compton length of the quark, $\lambda \propto 1 / m_{Q}$. This is not verified for the quark $b$ (as $a^{-1} \gtrsim(0.1 \mathrm{fm})^{-1} \sim 2 \mathrm{GeV}$ ) and on the lattice, heavy quarks have a mass of the order of $2 \mathrm{GeV}$ rather than the physical mass. Another approach can be used which consists in doing the calculation in the infinite mass limit. In this case, the heavy quark is static and propagates only in the time direction. Both calculations, with "light" $b$ quark and with infinite mass, can be made to obtain an interpolation to the physical $b$ quark mass. In this framework, the strange and charm quarks seem to be the easiest to simulate on the lattice, at present.

Lattice results for charm meson semileptonic decays involving a vector meson can be found in [43], [44] and [45]. They are compared with experimental results in Chapter 9.

\subsubsection{Different ansatze to parameterize hadronic form factors}

\section{Simple pole mass ansatz}

In the simple pole ansatz, the form factors are expected to be only governed by the spectroscopic pole corresponding to the $c \bar{s}$ lower mass state of quantum number $J^{P}=1^{+}$for the 
axial-vector current and $J^{P}=1^{-}$for the vector current ${ }^{4}$ :

$$
A_{i}\left(q^{2}\right)=\frac{A_{i}(0)}{1-q^{2} / M_{A}^{2}} \quad V\left(q^{2}\right)=\frac{V(0)}{1-q^{2} / M_{V}^{2}}
$$

with $M_{A}=2.5 \mathrm{GeV} / \mathrm{c}^{2}$ and $M_{V}=2.1 \mathrm{GeV} / \mathrm{c}^{2}$.

The distributions of the four kinematic variables for the $D_{s}^{+} \rightarrow \phi e^{+} \nu_{e}$ decay channel, using the simple pole model with $r_{2}=0.7$ and $r_{V}=1.5$, are given in Figure 1.12.
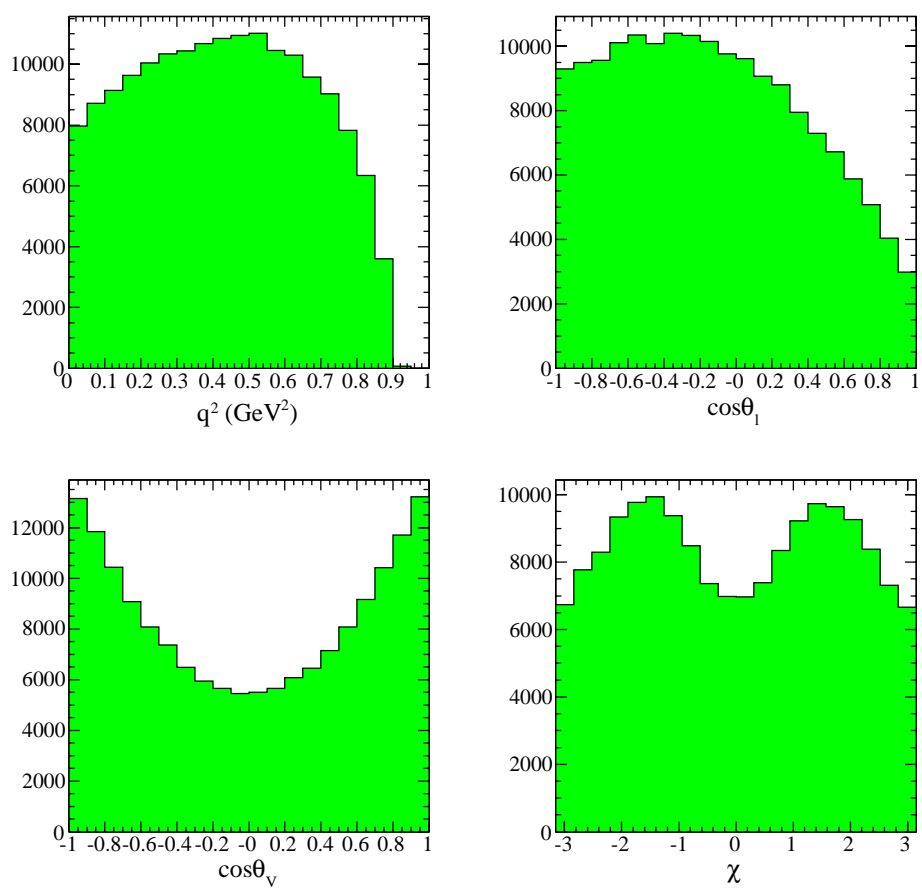

Figure 1.12: Distributions of the $D_{s}^{+} \rightarrow \phi e^{+} \nu_{e}$ differential decay rate versus $q^{2}, \cos \theta_{\ell}$, $\cos \theta_{V}$ and $\chi$ when the form factors are parameterized by the simple pole model. These plots are obtained using the BABAR simulation with following values of the parameters: $m_{A}=2.5 \mathrm{GeV} / \mathrm{c}^{2}, m_{V}=2.1 \mathrm{GeV} / \mathrm{c}^{2}, r_{2}=0.7$ and $r_{V}=1.5$.

It has been shown experimentally that pole models using nominal values of the pole masses do not give a good description of the form factor $q^{2}$ variation in the decay $D^{0} \rightarrow$ $K^{-} e^{+} \nu_{e}$ [46]. In this channel, the pole is expected to be situated at the $D_{s}^{*}$ mass $\left(2.112 \mathrm{GeV} / \mathrm{c}^{2}\right)$ whereas, using a simple pole expression, as in Eq. (1.82), the fitted pole mass value is lower: $m_{V}=1.884 \pm 0.012 \pm 0.015 \mathrm{GeV} / \mathrm{c}^{2}$. This discrepancy is not surprising because the approximation of the simple pole model is verified if the pole is well separated from the cut, so

\footnotetext{
${ }^{4}$ The $1^{-}$state contributing to $V$ is expected to be the $D_{s}^{*}$ of mass $2.112 \mathrm{GeV} / \mathrm{c}^{2}$ whereas the $1^{+}$states $D_{s J}(2459)$ and $D_{s 1}(2536)$ can contribute to $A_{1,2}$
} 
that the physical region of $q^{2}$ is only sensitive to the pole. From experiment, we know that the simple pole model has to be modified to include contributions from resonances higher in mass than the leading contribution. This is done in an ansatz proposed by Becirevic and Kaidalov for semileptonic decays into pseudo-scalar particles [47].

\section{Generalized Becirevic-Kaidalov ansatz}

The Becirevic-Kaidalov model [47] was developed for $B / D \rightarrow P \ell \nu_{\ell}$ decays, where $P$ is a light meson. In these decays, when the lepton mass is neglected, the differential decay rate depends on a single hadronic form factor $\left(f_{+}\left(q^{2}\right)\right)$. For $D \rightarrow K e \nu_{e}$, this vector form factor is expected to receive contributions from the $D_{s}^{*+}$ pole and from states situated at higher masses and having the same quantum numbers $\left(J^{P}=1^{-}\right)$. These components are supposed to generate a double pole behavior of the form factor:

$$
f_{+}\left(q^{2}\right)=\frac{c_{H}^{\prime}(1-a)}{\left(1-\frac{q^{2}}{m_{D_{s}^{*}}^{2}}\right)\left(1-a \frac{q^{2}}{m_{D_{s}^{*}}^{2}}\right)} .
$$

The value of the parameter $a$ is expected to be $\sim 0.5$.

This model has been generalized by Fajfer and Kamenik [48] to describe $B / D \rightarrow V \ell \nu_{\ell}$ decays. It is built in order to satisfy the scaling laws of HQET and LEET.

For $D_{s}^{+} \rightarrow \phi e^{+} \nu_{e}$, the three form factors are parameterized as:

$$
\begin{gathered}
V\left(q^{2}\right)=\frac{c_{H}^{\prime}(1-a)}{\left(1-\frac{q^{2}}{m_{D_{s}^{*}}^{2}}\right)\left(1-a \frac{q^{2}}{m_{D_{s}^{*}}^{2}}\right)}, \\
A_{1}\left(q^{2}\right)=\xi \frac{c_{H}^{\prime}(1-a)}{\left(1-b^{\prime} \frac{q^{2}}{m_{D_{s}^{*}}^{2}}\right)},
\end{gathered}
$$

and

$$
A_{2}\left(q^{2}\right)=\frac{c_{H}^{\prime \prime \prime}}{\left(1-b^{\prime} \frac{q^{2}}{m_{D_{s}^{*}}^{2}}\right)\left(1-b^{\prime \prime} \frac{q^{2}}{m_{D_{s}^{*}}^{2}}\right)} .
$$


In this model, $r_{V}$ is completely determined, in agreement with the expectation from LEET:

$$
r_{V}=\frac{1}{\xi}=\frac{\left(m_{D_{s}}+m_{\phi}\right)^{2}}{m_{D_{s}}^{2}+m_{\phi}^{2}}=1.8
$$

They provide also predictions for the parameter values based on experimental measurements of the meson masses and on $\mathrm{HM} \chi \mathrm{T}$ (Heavy Meson and Chiral Lagrangians) calculations. For the $D_{s}^{+} \rightarrow \phi e^{+} \nu_{e}$ channel:

$$
\begin{aligned}
V(0) & =1.10, A_{1}(0)=0.61, A_{2}(0)=0.32, \\
a & =0.57, a^{\prime}=0.53, b^{\prime}=0.74, b^{\prime \prime}=0 .
\end{aligned}
$$




\section{Chapter 2}

\section{The BABAR Experiment}

Suddenly she came upon a little three-legged table, all made of solid glass; there was nothing on it except a tiny golden key, and Alice's first thought was that it might belong to one of the doors of the hall; but, alas! either the locks were too large, or the key was too small, but at any rate it would not open any of them.

The study of the $D_{s}^{+} \rightarrow K^{+} K^{-} e^{+} \nu_{e}$ decay channel presented in this thesis has been done with the $B$ factory PEP-II and the BABAR detector. In this analysis, we use charm mesons produced in continuum $e^{+} e^{-} \rightarrow c \bar{c}$ events, the $B$ mesons are thus a source of background. The BABAR experiment has been optimized for the systematic study of $C P$-violation in the $B$ meson system. The geometry of the detector as well as the technical requirements of the main components have been designed in order to obtain the cleanest environment and the best efficiency to reconstruct the $B$ meson decays. The study of charm mesons in such conditions could be thought as difficult. In a certain way, this is true because we do not have a clean environment as in the CLEO-c experiment which is running at the charm production threshold. We have to deal with a consequent background as charm mesons are produced in the fragmentation process. But the great advantage of working in this experiment is the huge statistics of charm mesons available and dedicated studies can be developed to control the background. The performances of the detector, even optimized for $B$ physics, are also appropriate for the reconstruction of $c \bar{c}$ events.

$B A B A R$ is located at the Stanford Linear Accelerator Center (SLAC) in California. It involves a large international Collaboration of more than 500 physicists. The experiment 
consists of a detector [49] built around the interaction region of the high luminosity $e^{+} e^{-}$ asymmetric collider PEP-II [50]. In this chapter the main features of the final designs and the performances of PEP-II and the BABAR detector are described. The last section presents more specifically the particle identification tools used for the $D_{s}^{+} \rightarrow K^{+} K^{-} e^{+} \nu_{e}$ channel.

\subsection{The accelerator PEP-II}

The PEP-II $B$-Factory is an asymmetric-energy $e^{+} e^{-}$collider designed to operate at a center of mass energy of $10.58 \mathrm{GeV}$, corresponding to the mass of the $\Upsilon(4 S)$ vector meson resonance. The $\Upsilon(4 S)$ has a mass slightly above the $B \bar{B}$ threshold, and thus it decays almost exclusively into $B^{0} \bar{B}^{0}$ or $B^{+} B^{-}$pairs. When the $\Upsilon(4 S)$ is produced at rest, the small $B$ meson lifetime (about $1.5 \mathrm{ps}$ ) and the small boost provided to the $B$ mesons as a consequence of the vicinity of the $\Upsilon(4 S)$ mass to the $B \bar{B}$ threshold ${ }^{1}$ make experimentally very difficult to measure the separation of the two $B$ mesons decay points, which is necessary for the $C P$ asymmetries determination. By making an asymmetric-energy machine, with a $9.0 \mathrm{GeV}$ electron beam and a $3.1 \mathrm{GeV}$ positron beam, the $\Upsilon(4 S)$ receives a Lorentz boost of $\beta \gamma \simeq 0.56$, resulting in an average separation of about $250 \mu \mathrm{m}$ between the two $B$ vertices.

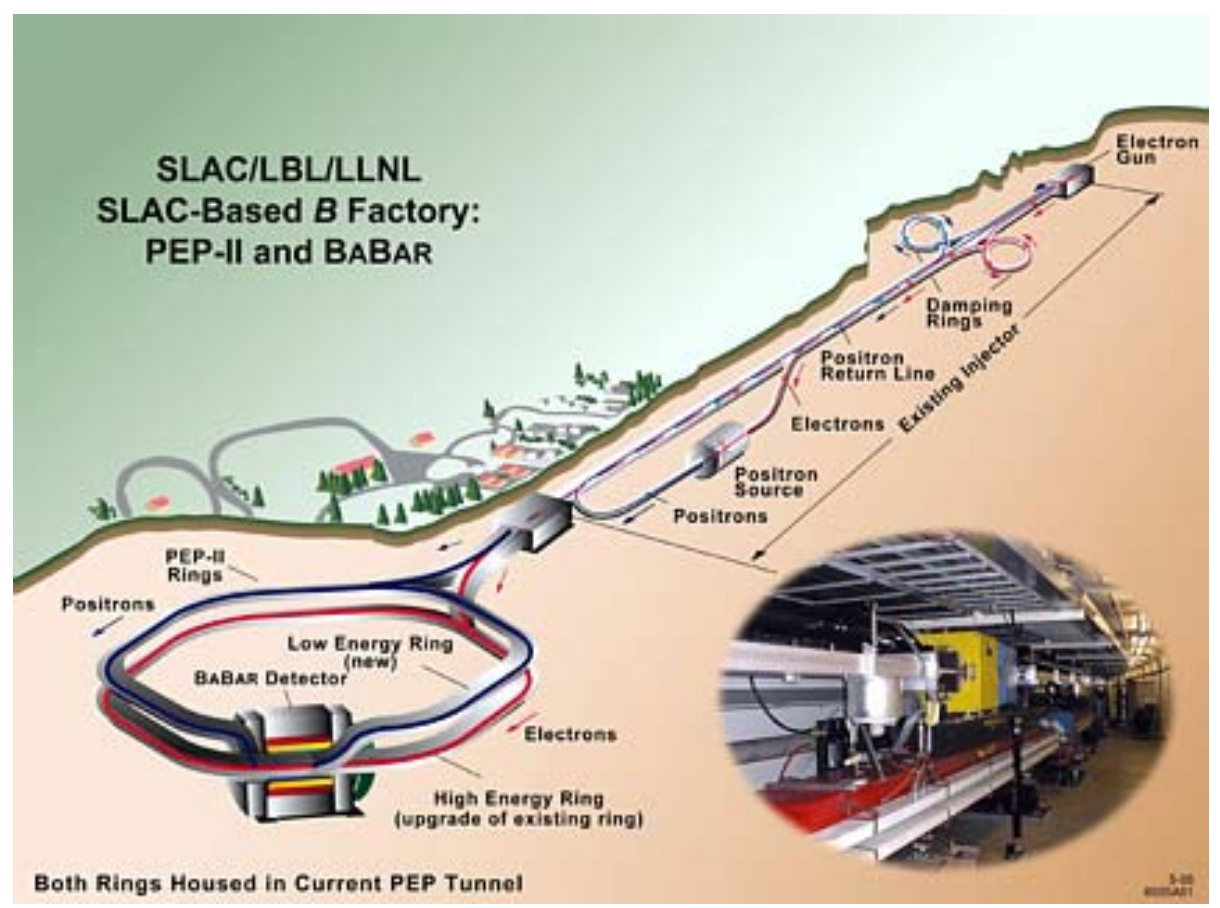

Figure 2.1: Overview of the PEP-II B-Factory.

\footnotetext{
${ }^{1}$ The $B$ mesons have a momentum of about $340 \mathrm{MeV} / c$ in the $\Upsilon(4 S)$ rest frame.
} 
An overview of the accelerator is shown in Figure 2.1. Electrons and positrons are accelerated from the $3.2 \mathrm{~km}$ long SLAC linac and accumulated into two $2.2 \mathrm{~km}$ long storage rings, called HER (high-energy ring, which contains the electrons) and LER (low-energy ring, which contains the positrons). Positrons are produced in the linac by collisions of $30 \mathrm{GeV}$ electrons to a target. In proximity of the interaction region the beams are focused by a series of offset quadrupoles (labelled Qx) and bent by means of a pair of dipole magnets, which allow the bunches to collide head-on and then to separate. The tapered B1 dipoles, located at $\pm 21 \mathrm{~cm}$ on each side of the interaction point (IP), and the Q1 quadrupoles operate inside the field of the BABAR superconducting solenoid, while Q2, Q4, and Q5, are located outside or in the fringe field of the solenoid (Figure 2.3). The interaction region is enclosed in a water-cooled beam pipe consisting of two thin layers of beryllium with a water channel in between. Its outer radius is about $28 \mathrm{~mm}$. The total thickness of the central beam pipe section at normal incidence corresponds to $1.06 \%$ of a radiation length. The beam pipe, the permanent magnets and the Silicon Vertex Tracker (SVT) are assembled, aligned and then enclosed in a $4.5 \mathrm{~m}$ long support tube. This rigid structure is inserted into the BABAR detector, spanning the IP.

\begin{tabular}{|l|c|}
\hline Event & Cross section (nb) \\
\hline \hline$b \bar{b}$ & 1.05 \\
\hline$c \bar{c}$ & 1.30 \\
\hline$s \bar{s}$ & 0.35 \\
\hline$u \bar{u}$ & 1.39 \\
\hline $\bar{d} \bar{d}$ & 0.35 \\
\hline$e^{+} e^{-}$ & $\sim 53$ \\
\hline$\mu^{+} \mu^{-}$ & 1.16 \\
\hline$\tau^{+} \tau^{-}$ & 0.94 \\
\hline
\end{tabular}

Table 2.1: Cross sections of the main physics processes at the $\Upsilon(4 S)$. The cross section for $e^{+} e^{-}$refers to events with one or both of the $e^{ \pm}$inside the electromagnetic calorimeter detection volume.

The cross sections of the main physics processes in PEP-II are listed in Tab. 2.1 [51]. At the peak of the $\Upsilon(4 S)$ there is a large amount of continuum $e^{+} e^{-} \rightarrow q \bar{q}(q=u, d, s$, $c)$ and $e^{+} e^{-} \rightarrow \ell \ell(\ell=e, \mu, \tau)$ events. Especially, charm is produced in $e^{+} e^{-} \rightarrow c \bar{c}$ events with a cross section of approximately $1.3 \mathrm{nb}$. To study specifically processes coming from continuum events, part of the data is collected at a center of mass energy $40 \mathrm{MeV}$ below the $\Upsilon(4 S)$ peak, where $B \bar{B}$ production is not allowed. This data sample corresponds to about $1 / 10$ of the sample taken at the $\Upsilon(4 S)$ peak.

Collisions in PEP-II started at the end of 1999, and since then BABAR has recorded an integrated luminosity of about $513 \mathrm{fb}^{-1}$, included about $48 \mathrm{fb}^{-1}$ of off-peak luminosity, and 
$433 \mathrm{fb}^{-1}$ recorded at the $\Upsilon(4 S)$. This dataset corresponds to more than one billion of charm decays. The BABAR recorded luminosity until march 2008 is shown in Figure 2.2.

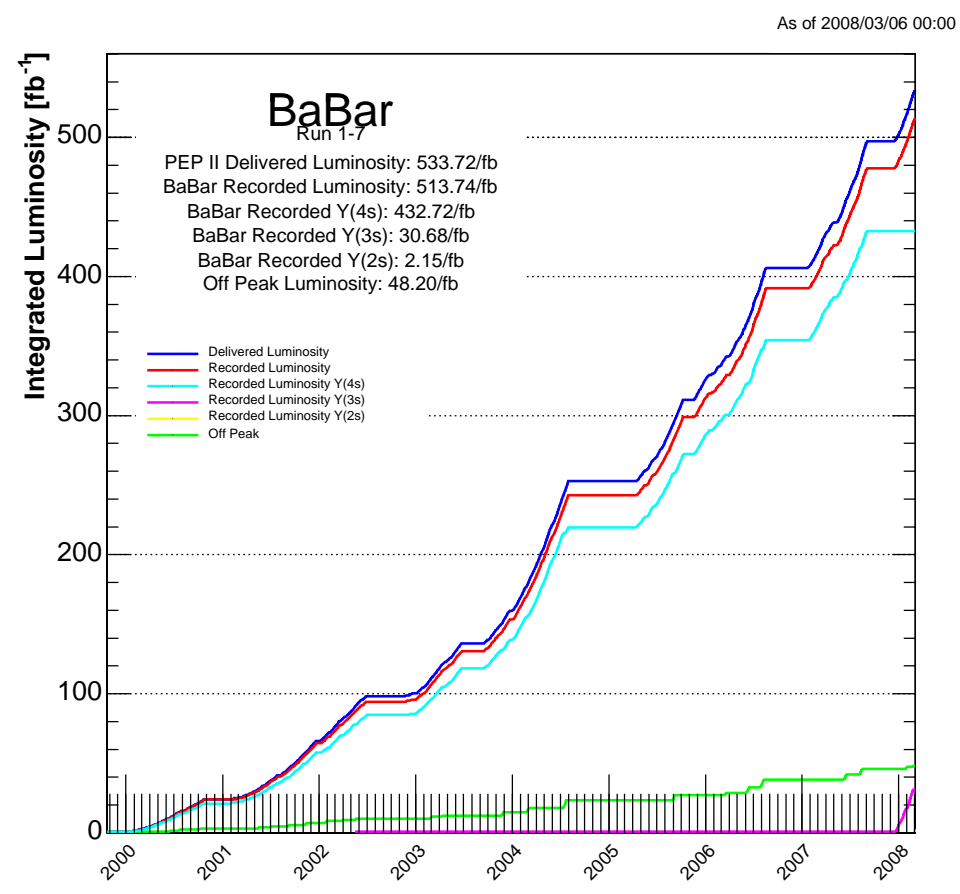

Figure 2.2: PEP-II delivered and BABAR recorded integrated luminosity in Run 1 to Run 7 (from October 1999 to March 2008).

\begin{tabular}{|lcc|}
\hline Parameters & Design & 2007 \\
\hline Energy HER/LER $(\mathrm{GeV})$ & $9.0 / 3.1$ & $9.0 / 3.1$ \\
Current HER/LER (A) & $0.75 / 2.15$ & $1.9 / 2.9$ \\
\# of bunches & 1658 & 1722 \\
Bunch length $(\mathrm{mm})$ & 15 & $11-12$ \\
Luminosity $\left(10^{33} \mathrm{~cm}^{-2} \mathrm{~s}^{-1}\right)$ & 3 & 12 \\
Integrated luminosity $\left(\mathrm{pb}^{-1} /\right.$ day $)$ & 135 & 911 \\
\hline
\end{tabular}

Table 2.2: PEP beam parameters. Values are given both for the design and for the records achieved during $200 \%$.

PEP-II surpassed its design performances, both in terms of the instantaneous luminosity and the daily integrated luminosity (see Table 2.2), achieving the peak value of $1.2 \times 10^{34} \mathrm{~cm}^{-2} \mathrm{~s}^{-1}$ during the Run 6 . A significant improvement to the integrated luminosity has been achieved between December 2003 and March 2004 with the implementation of a novel mode of operation of PEP-II, called "trickle injection". Until the end of 2003, PEP-II 
typically operated in a series of 40 minute fills during which the colliding beams coasted: at the end of each fill, it took about three to five minutes to replenish the beams for the next fill, and during this period the BABAR data acquisition system had to be turned off for detector safety. With the new technique, the BABAR detector can keep taking data virtually uninterrupted while the linac continuously injects electron and positron bunches (at a rate up to $10 \mathrm{~Hz}$ in the HER and $20 \mathrm{~Hz}$ in the LER) into the two PEP-II storage rings. This novel mode of operation allows an increase of 20 to $30 \%$ of the integrated luminosity. Moreover, the continuous injection makes the storage of particles more stable, so that PEP-II rings are easier to operate and beam losses are far less frequent than with the previous operational mode. This result is very important since, after a loss of the stored beams, it takes approximately 15 minutes to refill the two beams during which obviously no data taking is allowed.

The PEP-II high luminosity environment produces large backgrounds which can cause high single counting rates and currents in the detector components, radiation damages to electronics and sensors such as data acquisition dead times. This results in lower data quality and may limit the lifetime of the apparatus. For this reason the background generated by PEP-II has been studied in detail and the interaction region has been carefully designed. The primary sources of machine generated background [52] are the synchrotron radiation in the proximity of the interaction region, interactions between beam particles and residual gas in the rings and electromagnetic showers generated by beam-beam collisions. Background rates are continuously monitored during data acquisition to prevent critical operating conditions of the detector.

\subsection{The BABAR detector}

The design of the BABAR detector is optimized for $C P$ violation studies, but it is also well suited to do precision measurements in other $B$ and non $B$ physics. To achieve the goal of performing accurate measurements there are many requirements:

- a large and uniform acceptance, in particular down to small polar angles relative to the boost direction, to avoid particle losses. Although the boost originated by the asymmetric beams is not a big one, optimizing the detector acceptance leads to an asymmetric detector;

- a good vertex resolution;

- an excellent detection efficiency and an excellent precision on the momentum measurement for charged particles with transverse momentum ranging between $60 \mathrm{MeV} / c$ and 
$4 \mathrm{GeV} / c$;

- an excellent energy and angular resolution for photons and $\pi^{0} \mathrm{~s}$ with energy down to $20 \mathrm{MeV}$ and up to $5 \mathrm{GeV}$;

- a good discrimination between $e, \mu, \pi, K, p$ over a wide kinematic range;

- neutral hadrons identification capability.

Since the average momentum of charged particles produced in $B$ meson decays is below $1 \mathrm{GeV} / c$, the errors on the measured track parameters are dominated by multiple Coulomb scattering, rather than intrinsic spatial resolution of the detectors. Similarly, the detection efficiency and energy resolution of low energy photons are severely impacted by material in front of the calorimeter. Thus, special care has been given to keep the material in the active volume of the detector to a minimum.

A schematic view of the BABAR detector is shown in Figure 2.3. The BABAR superconducting solenoid, which produces a $1.5 \mathrm{~T}$ axial magnetic field, contains a set of nested detectors, which are - going from inside to outside - a five layers Silicon Vertex Tracker (SVT), a central Drift Chamber (DCH) for charged particles detection and momentum measurement, a fused-silica Cherenkov radiation detector (DIRC) for particle identification, and a $\mathrm{CsI}(\mathrm{Tl})$ crystal electromagnetic calorimeter for detection of photons and electrons. The calorimeter has a barrel and an end-cap which extends it asymmetrically into the forward direction ( $e^{-}$beam direction), where many of the collision products emerge. All the detectors located inside the magnet have practically full acceptance in azimuth $(\phi)$. The flux return outside the cryostat is composed of 18 layers of steel, which increase in thickness outwards, and are instrumented (the IFR) with 19 layers of planar resistive plate chambers (RPCs) or limited streamer tubes (LSTs) in the barrel and 18 in the end-caps. The IFR allows the muon identification, and also detects penetrating neutral hadrons. The right-handed coordinate system is indicated in Figure 2.3. The $\mathrm{z}$ axis corresponds to the magnetic field axis and is offset relative to the beam axis by about $20 \mathrm{mrad}$ in the horizontal plane. It is oriented in the direction of electrons. The positive $y$-axis points upward and the positive $x$-axis points away from the center of the PEP-II storage rings. The next Sections are dedicated to a description of each subsystem.

\subsubsection{The Silicon Vertex Tracker}

The Silicon Vertex Tracker (SVT) provides a precise measurement of the decay vertices and of the charged particle trajectories near the interaction region. The mean vertex resolution along the $z$-axis for a fully reconstructed $B$ decay must be better than $80 \mu \mathrm{m}$ in order to 

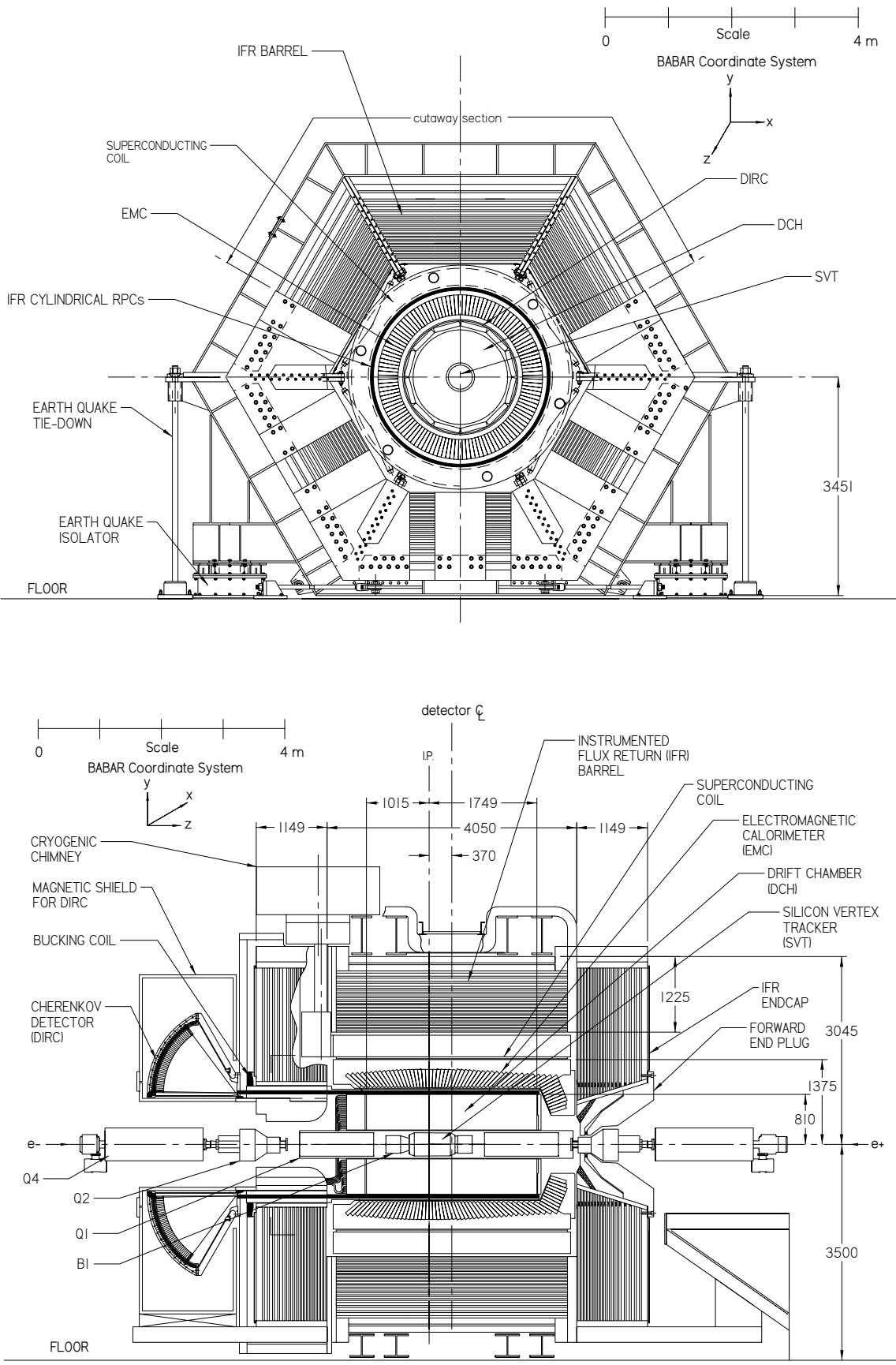

Figure 2.3: BABAR detector front view (top) and side view (bottom). 
avoid a significant impact on the time-dependent $C P$ asymmetry measurement precision; a $100 \mu \mathrm{m}$ resolution in the $x-y$ transverse plane is necessary in reconstructing decays of bottom and charm mesons, as well as $\tau$ leptons. The SVT also provides standalone tracking for particles with transverse momentum too low to reach the drift chamber, like soft pions from $D^{*}$ decays and many charged particles produced in multi-body $B$ meson decays. Finally, the SVT supplies particle identification (PID) information both for low and high momentum tracks. For low momentum tracks the SVT $d E / d x$ measurement is the only PID information available, for high momentum tracks the SVT provides the best measurement of the track angles, required to achieve the design resolution on the Cherenkov angle measured by the DIRC.

The design of the SVT is constrained by the components of the storage ring which have been arranged so as to allow maximum SVT coverage in the forward direction: the SVT extends down to $20^{\circ}\left(30^{\circ}\right)$ in polar angle from the beam line in the forward (backward) direction. Furthermore, it must have a small amount of material, so to reduce the multiple scattering which would affect the performance of the outer subdetectors. The solution which was adopted is a five-layer device with 340 double-sided silicon wafers mounted on a carbonfiber frame (Figures 2.4 and 2.5). On the inner (outer) face of each wafer, strip sensors are located running orthogonal (parallel) to the beam direction, measuring the $z(\phi)$ coordinate of the tracks. The wafers are organized in modules split into forward and backward sections: they are read out on their respective ends and the charge deposited by a particle is determined by the time over threshold of the signal on each strip. In total, 150,000 read-out channels are present. The inner three layers, containing six modules each, are placed close to the beam pipe (at 3.3, 4 and $5.9 \mathrm{~cm}$ from it) and dominate the determination of tracks position and angles. The outer two layers, containing 16 and 18 modules respectively, are arch-shaped, thus minimizing the amount of silicon needed to cover the solid angle, and placed close to the $\mathrm{DCH}$ (between 9.1 and $14.6 \mathrm{~cm}$ from the beam pipe) to help the track matching between the two detectors.

The total active silicon area is $0.96 \mathrm{~m}^{2}$ and the geometrical acceptance is $90 \%$ of the solid angle in the center-of-mass frame. The material traversed by particles corresponds to $\sim 4 \%$ of a radiation length.

The SVT efficiency is calculated for each section of the modules by comparing the number of associated hits to the number of tracks crossing the active area of the module and is found to be $97 \%$. The spatial resolution of SVT hits is determined by measuring the distance between the track trajectory and the hit for high-momentum tracks in two-prong events: it is generally better than $40 \mu \mathrm{m}$ in all layers for all track angles, allowing a precise determination of decay vertices to better than $70 \mu \mathrm{m}$. 


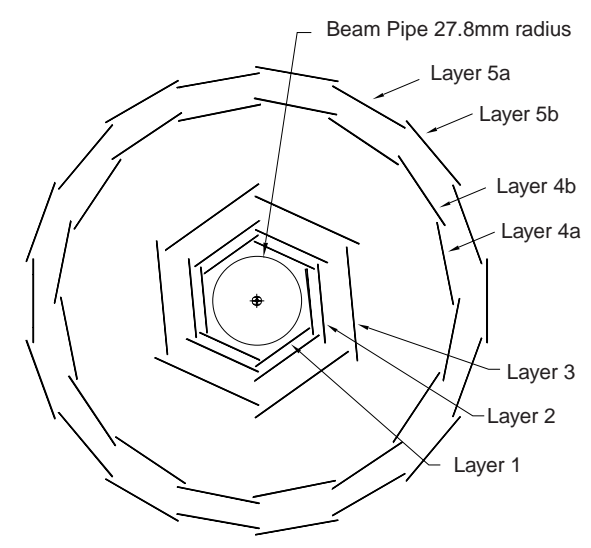

Figure 2.4: Schematic view of the SVT (transverse section).

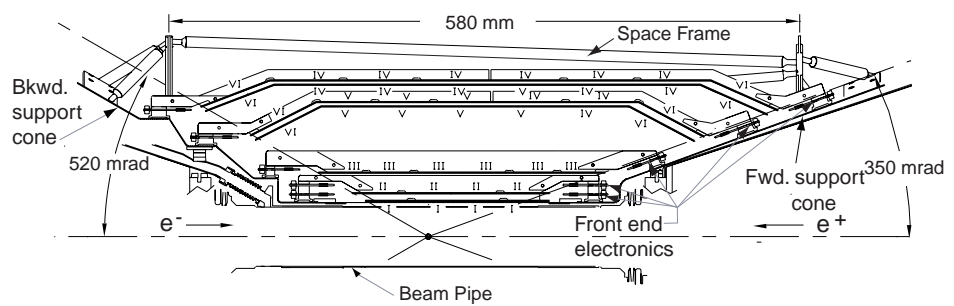

Figure 2.5: Schematic view of the SVT (longitudinal section).

The SVT provides stand-alone tracking for low momentum particles that do not reach the drift chamber. A comparison of the detected slow pion spectrum with the Monte Carlo prediction is presented in Figure 2.6 [49]. Based on this very good agreement the detection efficiency is estimated to be $20 \%$ for particles with transverse momenta of $50 \mathrm{MeV} / c$, rapidly increasing to over $80 \%$ at $70 \mathrm{MeV} / \mathrm{c}$.

Limited particle ID information for low momentum particles that do not reach the drift chamber and the Cherenkov detector is provided by the SVT through the measurement of the specific ionization loss, $d E / d x$, as derived from the total charge deposited in each silicon layer. The SVT $d E / d x$ distribution as a function of momentum is shown in Figure 2.6. The resolution achieved to date is typically about $14 \%$ for minimum ionizing particles, and a $2 \sigma$ separation between kaons and pions can be achieved up to momenta of $500 \mathrm{MeV} / \mathrm{c}$. 

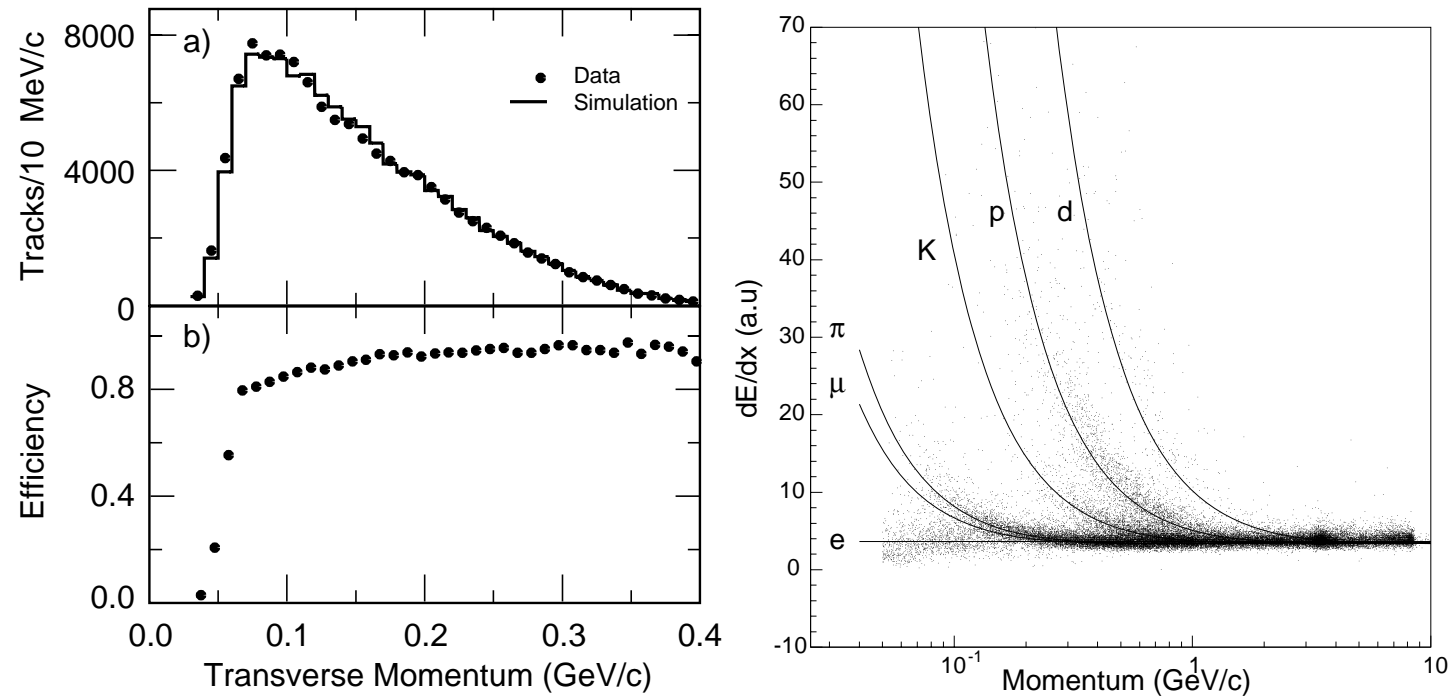

Figure 2.6: SVT detector performances: (a)Data-Monte Carlo comparison of the transverse momentum spectrum of soft pions in $D^{*+} \rightarrow D^{0} \pi^{+}$(top), and efficiency for slow pions detection estimated from simulated events (bottom). (b) Energy loss per unit length (dE/dx) as measured in the SVT as a function of momentum. The vertical scale is arbitrary

\subsubsection{The Drift Chamber}

The Drift Chamber (DCH) is the main tracking device for charged particles with transverse momenta $p_{T}$ above $\sim 120 \mathrm{MeV} / c$, providing the measurement of $p_{T}$ from the curvature of the particle's trajectory inside the $1.5 \mathrm{~T}$ solenoidal magnetic field. The DCH also allows the reconstruction of secondary vertices located outside the silicon detector volume, such as those from $K_{S}^{0} \rightarrow \pi^{+} \pi^{-}$decays. For this purpose, the chamber is able to measure not only the transverse coordinate, but also the longitudinal $(z)$ position of tracks with good resolution (about $1 \mathrm{~mm}$ ). Good $z$ resolution also aids in matching DCH and SVT tracks, and in projecting tracks to the DIRC and the calorimeter. For low momentum particles the DCH provides particle identification by measurement of ionization loss $(d E / d x)$, thus allowing for $K / \pi$ separation up to $\approx 700 \mathrm{MeV} / c$. This capability is complementary to that of the DIRC in the barrel region, while it is the only mean to discriminate between different particle hypotheses in the extreme backward and forward directions which fall outside of the geometric acceptance of the DIRC. Finally, the DCH provides real-time information used in the first level trigger system.

The DCH is a $2.80 \mathrm{~m}$ long cylinder with an inner radius of $23.6 \mathrm{~cm}$ and an outer radius of $80.9 \mathrm{~cm}$ (Figure 2.7). Given the asymmetry of the beam energies, the DCH center is displaced by about $37 \mathrm{~cm}$ with respect to the interaction point in the forward direction. The active volume provides charged particle tracking over the polar angle range $-0.92<\cos \theta<0.96$. 


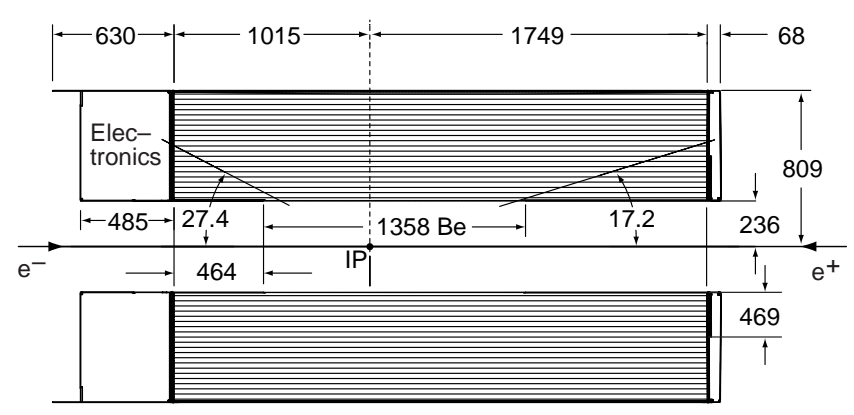

Figure 2.7: Schematic view of the DCH (longitudinal section).

The drift system consists of 7104 hexagonal cells, approximately $1.8 \mathrm{~cm}$ wide by $1.2 \mathrm{~cm}$ high, arranged in 10 superlayers of 4 layers each, for a total of 40 concentric layers (Fig. 2.8). Each cell consists of one sense wire surrounded by six field wires. The sense wires are $20 \mu \mathrm{m}$ Rh-W gold-plated wires operating nominally in the range 1900-1960 V; the field wires are $120 \mu \mathrm{m} \mathrm{Al}$ wires operating at $340 \mathrm{~V}$. Within a given superlayer, the sense and field wires are organized with the same orientation. For measuring also the $z$ coordinate, the superlayers alternate in orientation: first an axial view, then a pair of small angle stereo views (one with positive, one with negative angle), as indicated in Fig. 2.8.

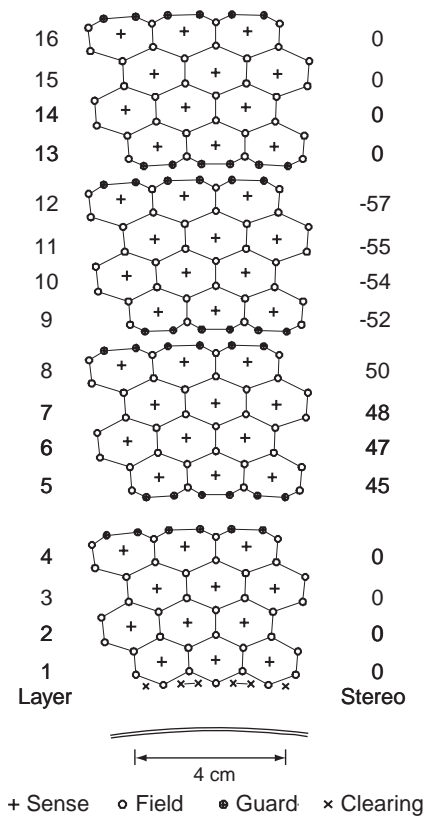

Figure 2.8: Schematic layout of the drift cells for the four innermost superlayers. The numbers on the right side give the stereo angles (mrad) of sense wires in each layer. 

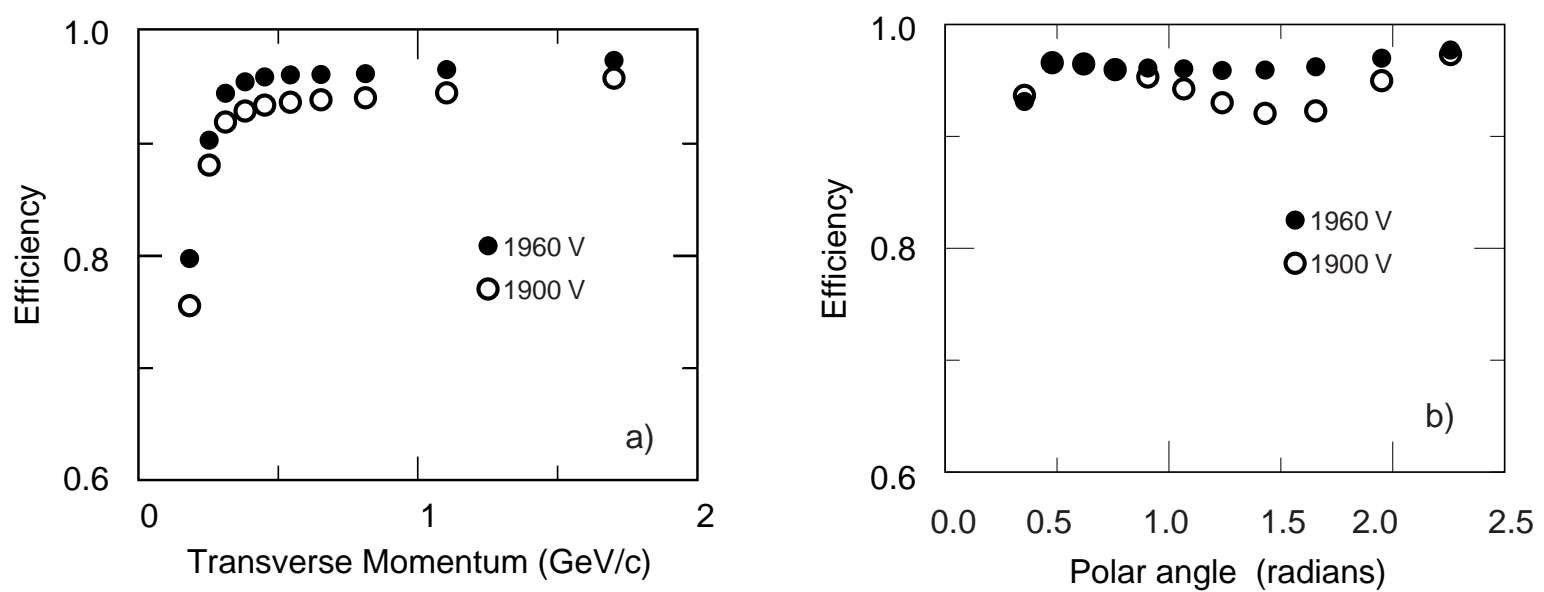

Figure 2.9: Track reconstruction efficiency in the drift chamber at operating voltages of 1900 $V$ and $1960 \mathrm{~V}$, as a function of transverse momentum (a) and polar angle (b).

The layers are housed between a $1 \mathrm{~mm}$ beryllium inner wall and a $9 \mathrm{~mm}$ carbonfiber outer wall (corresponding to $0.28 \%$ and $1.5 \%$ radiation lengths, respectively) both to facilitate the matching between the SVT and DCH tracks and to minimize the amount of material in front of the DIRC and the calorimeter. The counting gas is a 80:20 mixture of helium: isobutane, which again satisfies the requirement of keeping the multiple scattering at minimum. Overall, the multiple scattering inside the DCH is limited by less than $0.2 \%$ radiation lengths of material.

The drift chamber reconstruction efficiency has been measured on data in selected samples of multi-track events by exploiting the fact that tracks can be reconstructed independently in the SVT and the DCH. The absolute drift chamber tracking efficiency is determined as the fraction of all tracks detected in the SVT which are also reconstructed by the DCH when they fall within its acceptance. Its dependency on the transverse momentum and polar angle is shown in Fig. 2.9 [49]. At the design voltage of $1960 \mathrm{~V}$ the reconstruction efficiency of the drift chamber averages $98 \pm 1 \%$ for tracks above $200 \mathrm{MeV} / c$ and polar angle $\theta>500 \mathrm{mrad}$ $\left(29^{\circ}\right)$.

The $p_{T}$ resolution is measured as a function of $p_{T}$ in cosmic ray studies:

$$
\frac{\sigma_{p_{T}}}{p_{T}}=(0.13 \pm 0.01) \% \cdot p_{T}+(0.45 \pm 0.03) \%,
$$

where $p_{T}$ is expressed in $\mathrm{GeV} / c$. The first contribution, dominating at high $p_{T}$, comes from the curvature error due to finite spatial measurement resolution; the second contribution, dominating at low momenta, is due to multiple Coulomb scattering. 

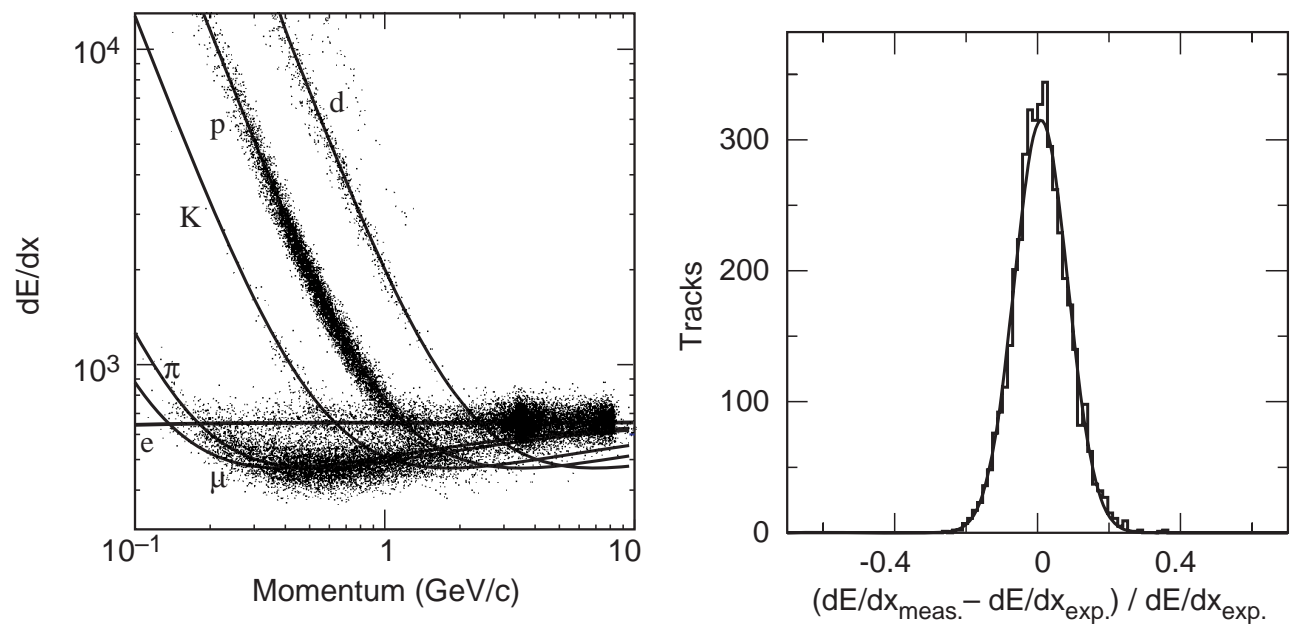

Figure 2.10: Left: reconstructed $d E / d x$ as a function of track momenta. Right: resolution on $d E / d x$ for $e^{ \pm}$from Bhabha scattering.

The specific ionization loss $d E / d x$ for charged particles traversing the drift chamber is derived from the total charge deposited in each drift cell. The left plot of Figure 2.10 shows the distribution of the reconstructed and corrected $d E / d x$ from the drift chamber as a function of track momenta. The superimposed Bethe-Bloch curves for the individual particle species have been determined using various particle control samples. The resolution achieved to date is typically about $7.5 \%$ (as shown in the right plot of Fig. 2.10 for $e^{ \pm}$from Bhabha scattering). A $3 \sigma$ separation between kaons and pions can be achieved up to momenta of about $700 \mathrm{MeV} / \mathrm{c}[53]$.

\subsubsection{The Cherenkov detector}

The particle identification (PID) at low momenta exploits primarily the $d E / d x$ measurements in the DCH and SVT. However, above the threshold of $700 \mathrm{MeV} / c$, the $d E / d x$ information does not allow to separate pions and kaons. The Detector of Internally Reflected Cherenkov radiation (DIRC) is employed primarily for the separation of pions and kaons from about 500 $\mathrm{MeV} / c$ to the kinematic limit of $4 \mathrm{GeV} / c$ reached in rare $B$ decays like $B \rightarrow \pi^{+} \pi^{-} / K^{+} K^{-}$. The principle of the DIRC is based on the detection of Cherenkov light generated by a charged particle in a medium of refractive index $n$, when its velocity $v$ is greater than $c / n$. The photons are emitted on a cone of half-angle $\theta_{c}$ with respect to the particle direction, where $\cos \theta_{c}=1 / \beta n, \beta=v / c$. Knowing the particle momentum thanks to the SVT and the $\mathrm{DCH}$, the measurement of $\theta_{c}$ allows the mass measurement, so the particle identification, 
with the relation:

$$
m^{2} c^{2}=\frac{1-\beta^{2}}{\beta^{2}} p^{2}
$$

Figure 2.11 illustrates the principles of light production, transport, and imaging.

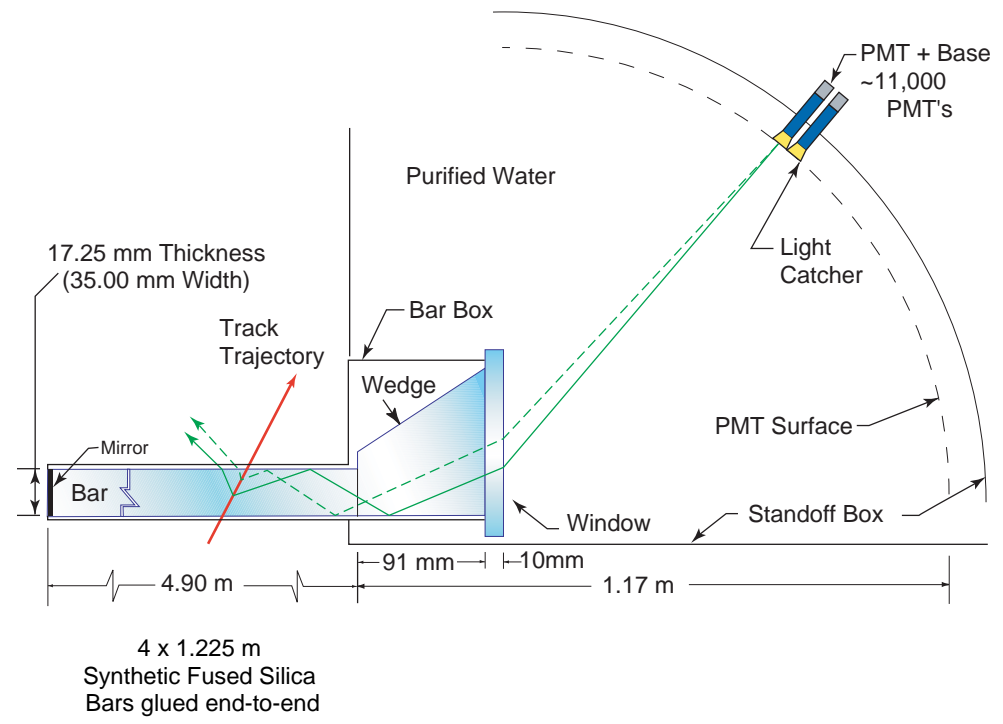

Figure 2.11: Schematics of the DIRC fused silica radiator bar and imaging region.

The radiator material of the DIRC is synthetic fused silica (refraction index $n=1.473$ ) in the form of 144 long, thin bars with regular rectangular cross section. The bars, which are $17 \mathrm{~mm}$ thick, $35 \mathrm{~mm}$ wide and $4.9 \mathrm{~m}$ long, are arranged in a 12-sided polygonal barrel, each side being composed of 12 adjacent bars placed into sealed containers called bar boxes. Dry nitrogen gas flows through each bar box, and humidity levels are measured to monitor that the bar box to water interface remains sealed. The solid angle subtended by the radiator bars corresponds to $94 \%$ of the azimuth and $83 \%$ of the cosine of the polar angle in the center-of-mass system. The bars serve both as radiators and as light pipes for the portion of the light trapped in the radiator by total internal reflection. For particles with $\beta \approx 1$, some photons will always lie within the total internal reflection limit, and will be transported to either one or both ends of the bar, depending on the particle incident angle. To avoid having to instrument both bar ends with photon detectors, a mirror is placed at the forward end, perpendicular to the bar axis, to reflect incident photons to the backward (instrumented) bar end.

Once photons arrive at the instrumented end, most of them emerge into an expansion region filled with 6000 litres of purified water $(n=1.346)$, called the stand-off box (see Figure 2.12). A fused silica wedge at the exit of the bar reflects photons at large angles and 
thereby reduces the size of the required detection surface. The photons are detected by an array of densely packed photo-multiplier tubes (PMTs), each surrounded by reflecting "light catcher" cones to capture light which would otherwise miss the PMT active area. The PMTs, arranged in 12 sectors of 896 phototubes each, have a diameter of $29 \mathrm{~mm}$ and are placed at a distance of about $1.2 \mathrm{~m}$ from the bar end. The expected Cherenkov light pattern at this surface is essentially a conic section, whose cone opening-angle is the Cherenkov production angle modified by refraction at the exit from the fused silica window. By knowing the location of the PMT that observes a Cherenkov photon and the charged particle direction from the tracking system, the Cherenkov angle can be determined. In addition, the time taken for the photon to travel from its point of origin to the PMT is used to effectively suppress hits from beam-generated background and from other tracks in the same event, and also to resolve some ambiguities in the association between the PMT hits and the track (for instance, the forward-backward ambiguity between photons that have or haven't been reflected by the mirror at the forward end of the bars).

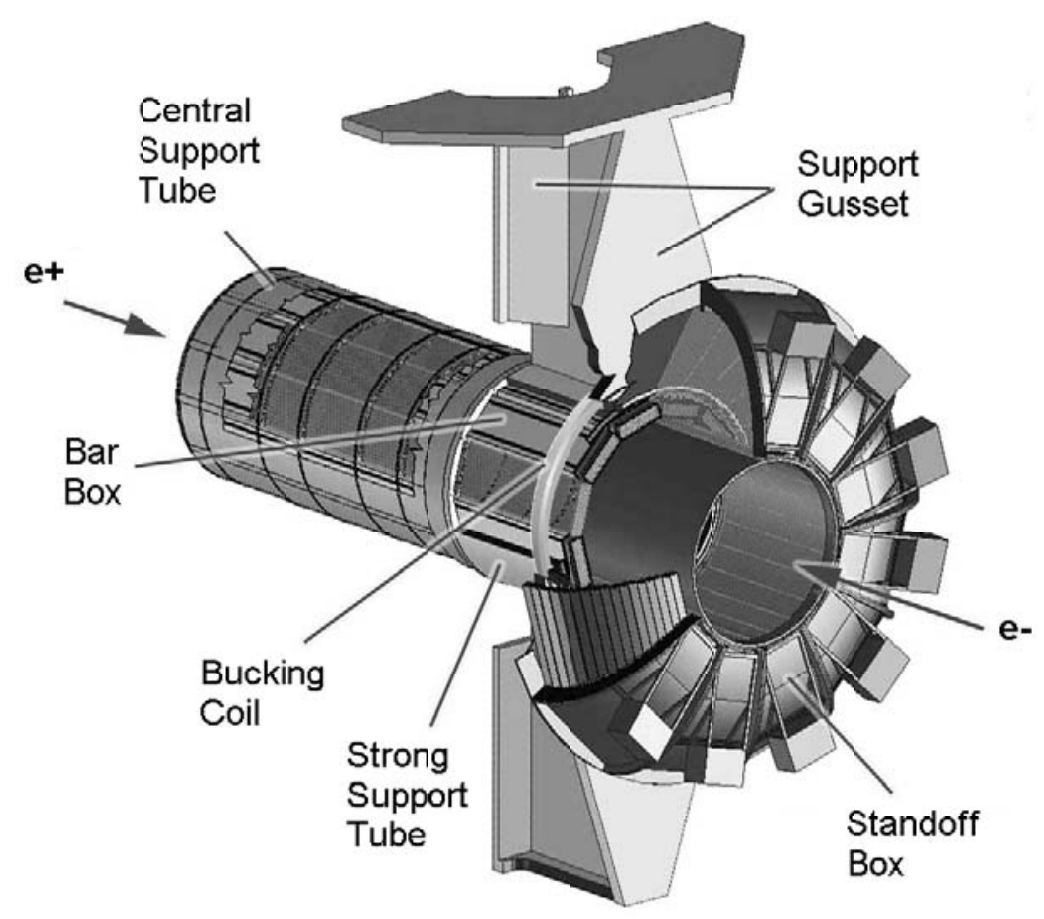

Figure 2.12: Schematic view of the principal components of the DIRC mechanical support structure.

The relevant observable to distinguish between signal and background photons is the difference between the measured and expected photon time, $\delta t_{\gamma}$. It is calculated for each photon using the track time-of-flight, the measured time of the candidate signal in the PMT 
and the photon propagation time within the bar and the water filled standoff box. The resolution on this quantity, as measured in dimuon events is $1.7 \mathrm{~ns}$, close to the intrinsic 1.5 ns transit time spread of the photoelectrons in the PMTs. Applying the time information substantially improves the correct matching of photons with tracks and reduces the number of accelerator induced background hits by approximately a factor 40 , as can be seen in Figure 2.13 [54]. The reconstruction routine provides a likelihood value for each of the five stable particle types $(e, \mu, \pi, K, p)$ if the track passes through the active volume of the DIRC. These likelihoods are calculated in an iterative process by maximising the likelihood value for the entire event while testing different hypotheses for each track. If enough photons are found, a fit of $\theta_{c}$ and the number of observed signal and background photons are calculated for each track.
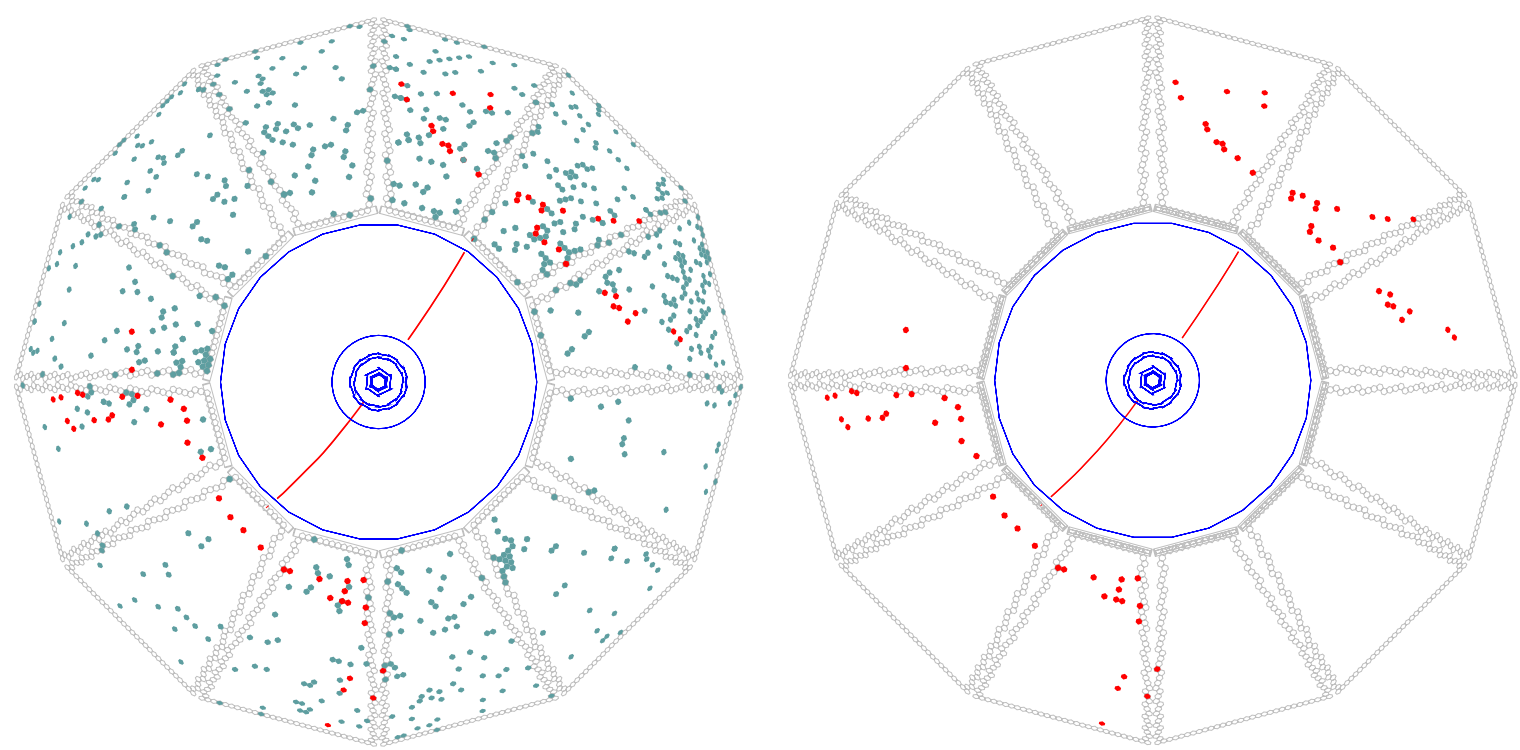

Figure 2.13: Display of one $e^{+} e^{-} \rightarrow \mu^{+} \mu^{-}$event reconstructed in BABAR with two different time cuts. On the left, all DIRC PMTs that were hit within the \pm 300 ns trigger window are shown. On the right, only those PMTs that were hit within 8 ns of the expected Cherenkov photon arrival time are displayed.

The DIRC uses two independent approaches for a calibration of the unknown PMT time response and the delays introduced by the electronic and the fast control system. The first one is a conventional pulser calibration: 1 ns duration light pulses are emitted from 12 blue LEDs (one per sector), with a rate of $2 \mathrm{kHz}$. A calibration run requires a few minutes and is taken about three times a week. The second calibration system uses reconstructed tracks from the collision data ("rolling calibration"). It performs a calibration of the global time delay, and the time delay sector by sector.

Figure 2.14 shows the number of photons detected as a function of the polar angle in di- 


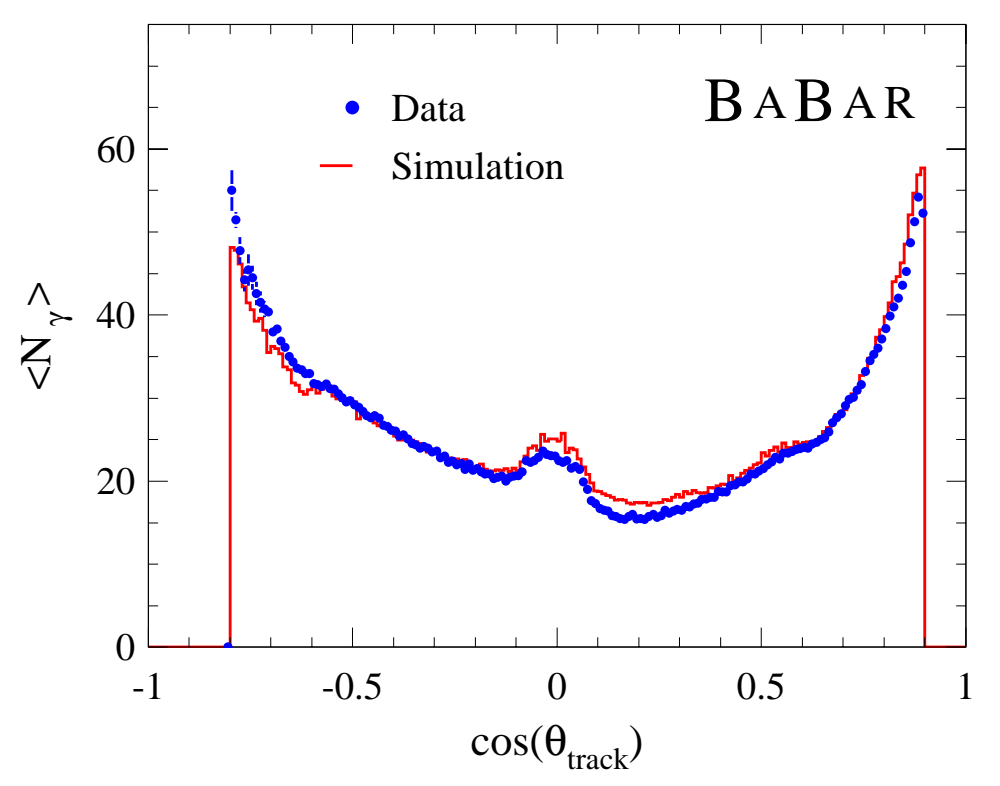

Figure 2.14: Number of detected photoelectrons versus track polar angle for reconstructed di-muon events in data and simulation.

muons events. It increases from a minimum of about 20 at the center of the barrel $\left(\theta \approx 90^{\circ}\right)$ to well over 50 in the forward and backward directions, corresponding to the fact that the pathlength in the radiator is longer for tracks emitted at large dip angles (therefore the number of Cherenkov photons produced in the bars is greater) and the fraction of photons trapped by total internal reflection rises. This feature is very useful in the BABAR environment, where, due to the boost of the center-of-mass, particles are emitted preferentially in the forward direction. The bump at $\cos \theta=0$ is a result of the fact that for tracks at small angles internal reflection of the Cherenkov photons occurs in both the forward and backward direction. The small decrease of the number of photons from the backward direction to the forward one is a consequence of the photon absorption along the bar before reaching the stand-off box in the backward end. The combination of the single photon Cherenkov angle resolution, the distribution of the number of detected photons versus polar angle and the polar angle distribution of charged tracks yields a typical track Cherenkov angle resolution which is about $2.5 \mathrm{mrad}$ in di-muon events. The $D^{*} \rightarrow D^{0} \pi, D^{0} \rightarrow K \pi$ decay chain is well suited to probe the pion and kaon identification capabilities of the DIRC. It is kinematically well constrained and the momentum spectrum of the charged pions and kaons covers the range accessible by $B$ meson decay products in BABAR. The pion-kaon separation power is defined as the difference of the mean Cherenkov angles for pions and kaons assuming a Gaussian-like distribution, divided by the measured track Cherenkov angle resolution. As shown in Figure 2.15 , the separation between kaons and pions at $3 \mathrm{GeV} / c$ is about $4.3 \sigma$. 

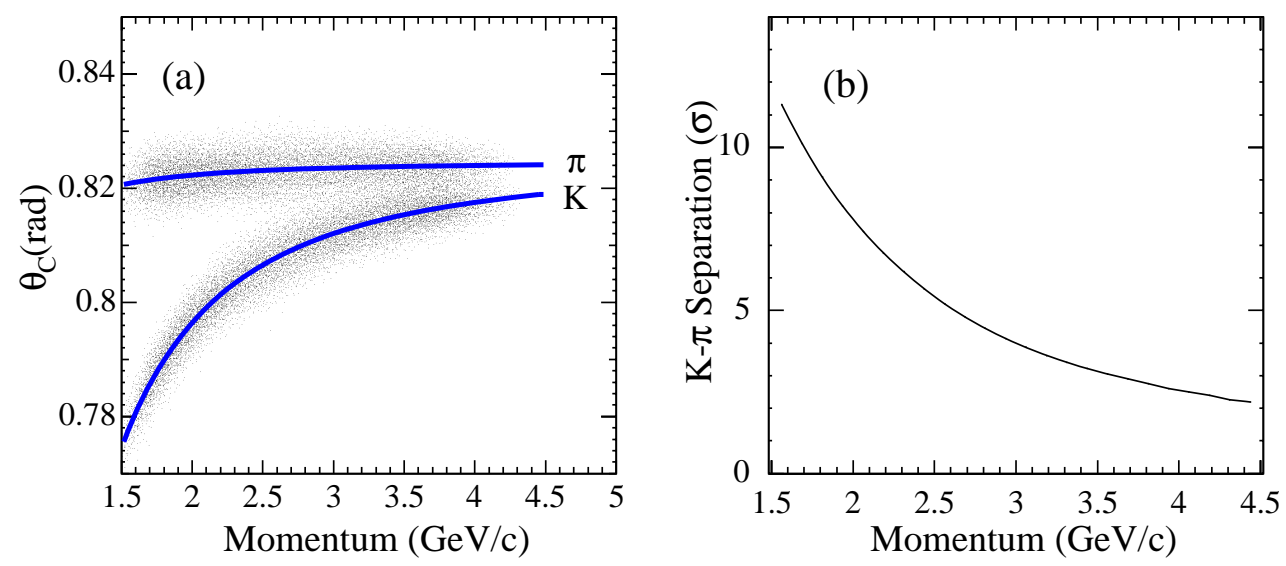

Figure 2.15: (a) The measured Cherenkov angle for pions (upper band) and kaons (lower band) from $D^{*} \rightarrow D^{0} \pi, D^{0} \rightarrow K \pi$ decays reconstructed in data. The curves show the expected angle $\theta_{C}$ as a function of laboratory momentum, for the $K$ and $\pi$ mass hypothesis. (b) The average difference between the expected value of $\theta_{C}$ for kaons and pions, divided by the uncertainty, as a function of momentum.

The efficiency for correctly identifying a charged kaon hitting a radiator bar and the probability of wrongly identifying a pion as a kaon are determined using $D^{0}$ decays kinematically selected from inclusive $D^{*}$ meson production (Figure 2.16): the kaon identification efficiency and pion mis-identification probability are about $96 \%$ and $2 \%$, respectively.

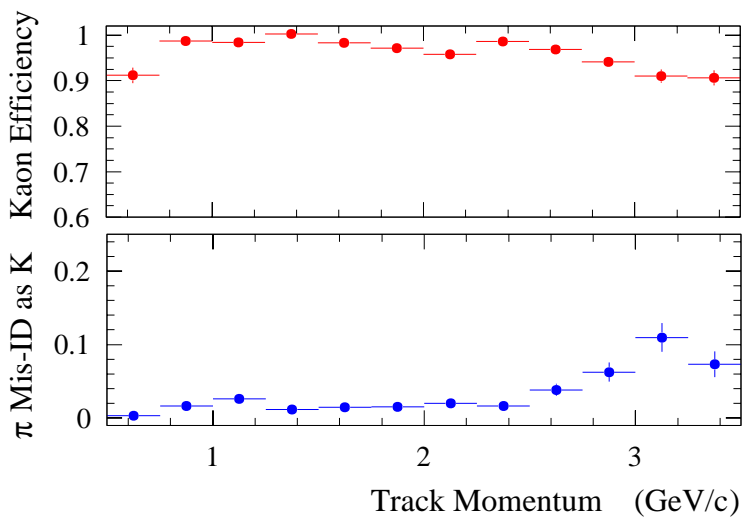

Figure 2.16: Efficiency and misidentification probability for the selection of charged kaons as a function of track momentum. 


\subsubsection{The Electromagnetic Calorimeter}

The BABAR electromagnetic calorimeter (EMC) is designed to detect and measure electromagnetic showers with high efficiency and very good energy and angular resolution over a wide energy range between $20 \mathrm{MeV}$ and $9 \mathrm{GeV}$. This allows the reconstruction of $\pi^{0} \rightarrow \gamma \gamma$ and $\eta \rightarrow \gamma \gamma$ decays where the photons can have very low energy, as well as the reconstruction of Bhabha events and processes like $e^{+} e^{-} \rightarrow \gamma \gamma$, important for luminosity monitoring and calibration, where the electron and photon energies can be as large as $9 \mathrm{GeV}$. The EMC also provides the primary information for electron identification and electron-hadron separation.

Energy deposit clusters in the EMC with lateral shape consistent with the expected pattern from an electromagnetic shower are identified as photons when they are not associated to any charged tracks extrapolated from the SVT and the drift chamber, and as electrons if they are matched to a charged track and if the ratio between the energy $E$ measured in the EMC and the momentum $p$ measured by the tracking system is $E / p \approx 1$.

The EMC contains 6580 CsI crystals doped with Tl (Figure 2.17). CsI(Tl) has a high light yield $(50,000$ photons $/ \mathrm{MeV})$ and a small Molière radius $(3.8 \mathrm{~cm})$, which provide the required energy and angular resolution; its radiation length of $1.86 \mathrm{~cm}$ guarantees complete shower containment at the BABAR energies.

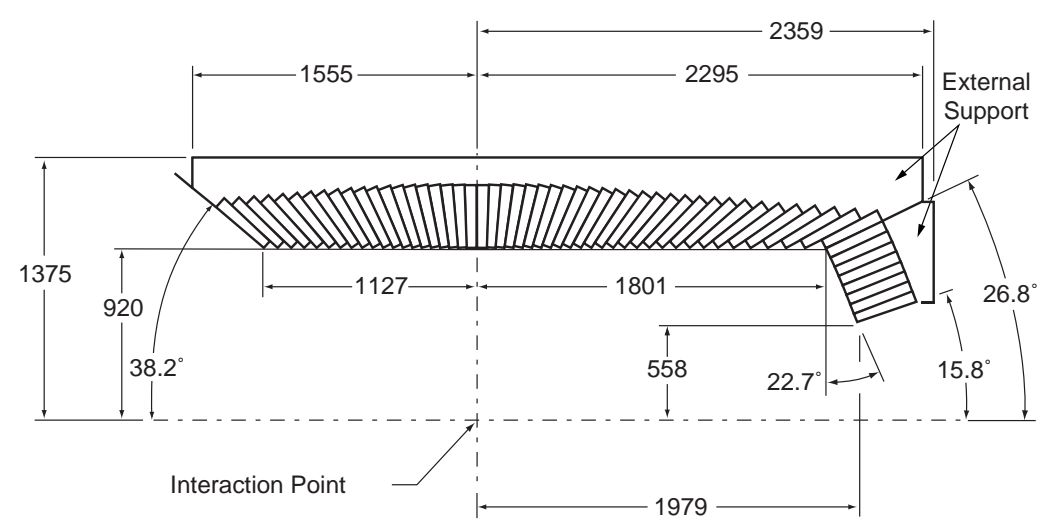

Figure 2.17: Longitudinal section of the top half of the EMC. Dimensions are in mm.

Each crystal is a truncated trapezoidal pyramid and ranges from 16 to 17.5 radiation lengths in thickness. The front faces are typically about $5 \mathrm{~cm}$ in each dimension. The crystals are arranged to form a barrel and a forward endcap giving a $90 \%$ solid-angle coverage in the center-of-mass frame. The barrel has 48 rows of crystals in $\theta$ and 120 in $\phi$; the forward endcap contains 8 rings in $\theta$. Overall the EMC extends from an inner radius of $91 \mathrm{~cm}$ to an outer radius of $136 \mathrm{~cm}$ and is displaced asymmetrically with respect to the interaction point. 
The crystals are read out by two independent $1 \mathrm{~cm}^{2}$ PIN photodiodes, glued to their rear faces, which are connected to low-noise preamplifiers that shape the signal with a short shaping time (400 ns) so to reduce soft beam-related photon backgrounds.

For the purpose of precise calibration and monitoring, use is made of a neutron activated fluorocarbon fluid, which produces a radioactive source $\left({ }^{16} N\right)$ originating a $6.1 \mathrm{MeV}$ photon peak in each crystal. A light pulser system injecting light into the rear of each crystal is also used. In addition, signals from data, including $\pi^{0}$ decays and $e^{+} e^{-} \rightarrow e^{+} e^{-} / \gamma \gamma / \mu^{+} \mu^{-}$ events, provide an energy calibration and resolution determination.

The efficiency of the EMC exceeds $96 \%$ for the detection of photons with energy above $20 \mathrm{MeV}$. The energy resolution is usually parameterized by

$$
\frac{\sigma_{E}}{E}=\frac{\sigma_{1}}{E^{1 / 4}(\mathrm{GeV})} \oplus \sigma_{2}
$$

where $\sigma_{1}=2.32 \pm 0.30 \%$ and $\sigma_{2}=1.85 \pm 0.12 \%$, as determined using the above mentioned sources. The first term in Eq. 2.3 arises from fluctuations in photon statistics and is dominant for energies below about $2.5 \mathrm{GeV}$, while the constant term takes into account several effects, such as fluctuations in shower containment, non-uniformities, calibration uncertainties and electronic noise.
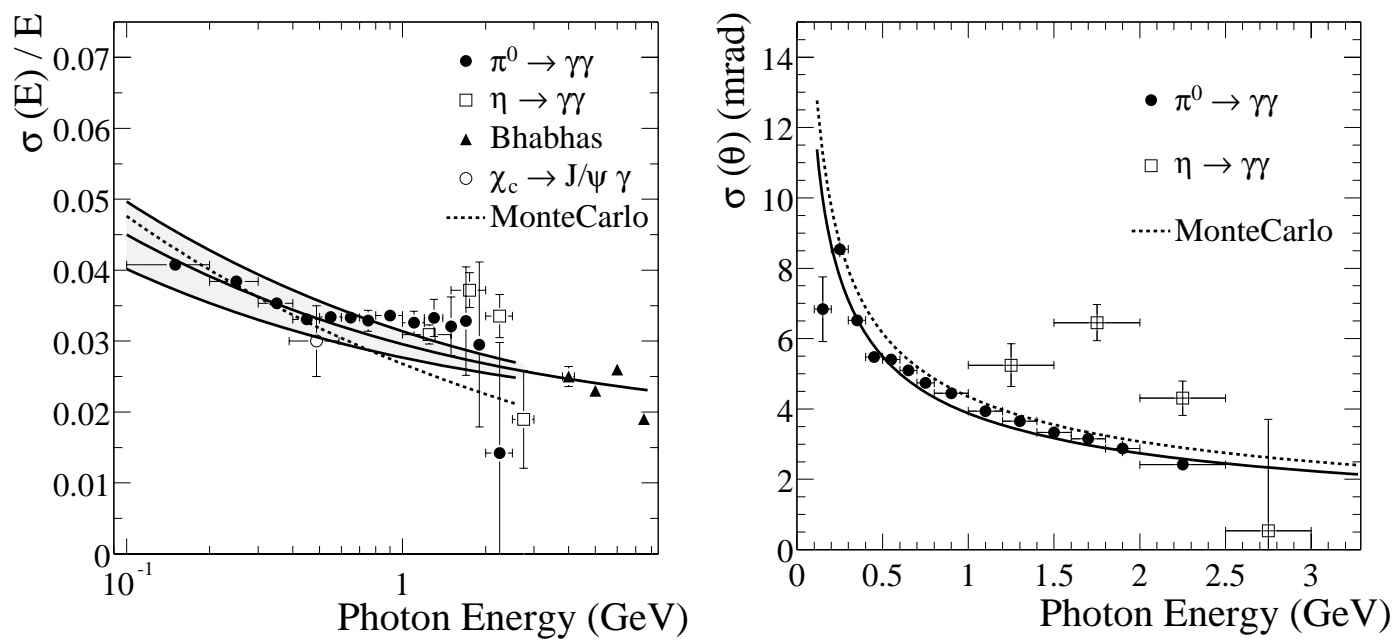

Figure 2.18: Energy (left) and angular (right) resolutions measured using a variety of data. The solid curves represent a fit to the data using Equation 2.3 and 2.4 respectively.

The decays of $\pi^{0}$ and $\eta$ candidates in which the two photons have approximately equal energy are used to infer angular resolution. It varies between about $12 \mathrm{mrad}$ at low energies 
and $3 \mathrm{mrad}$ at high energy. The data fit the empirical parameterization:

$$
\sigma_{\theta, \phi}=\left(\frac{(3.87 \pm 0.07)}{\sqrt{E(\mathrm{GeV})}}+(0.00 \pm 0.04)\right) \operatorname{mrad}
$$

Fig. 2.18 [55] shows the energy and angular resolution measured as a function of the photon energy.

\subsubsection{The Instrumented Flux Return}

The Instrumented Flux Return (IFR) is designed to identify muons and neutral hadrons (primarily $K_{L}$ and neutrons). Muons are important for tagging the flavor of neutral $B$ mesons via semi-leptonic decays, for the reconstruction of vector mesons, like the $J / \psi$, and the study of semi-leptonic and rare decays involving leptons from $B$ and $D$ mesons and $\tau$ leptons. $K_{L}$ detection allows for the study of exclusive $B$ decays, in particular $C P$ eigenstates. The principal requirements for IFR are large solid angle coverage, good efficiency and high background rejection for muons down to momenta below $1 \mathrm{GeV} / c$. For neutral hadrons, high efficiency and good angular resolution are most important. The IFR uses the steel flux return of the magnet as muon filter and hadron absorber, limiting pion contamination in the muon identification. Originally single gap resistive plate chambers (RPC) with two-coordinate readout, operated in limited streamer mode constituted the active part of the detector [56], with 19 layers in the barrel and 18 in each endcap. The RPC were installed in the gaps of the finely segmented steel of the six barrel sectors and the two end-doors of the flux return, as illustrated in Fig. 2.19. The steel segmentation has been optimized on the basis of Monte Carlo studies of muon penetration and charged and neutral hadron interactions. In addition, two layers of cylindrical RPCs were installed between the EMC and the magnet cryostat to detect particles exiting the EMC. RPCs contain a $2 \mathrm{~mm}$ Bakelite gap with $\sim 8 \mathrm{kV}$ across it. Ionizing particles which cross the gap create streamers of ions and electrons in the gas mixture (Argon, freon and isobutane), which in turn creates signals via capacitive coupling on the strips mounted on each side of the RPC. Soon after the installation (which took place in Summer 1999), the efficiency of a significant fraction of the chambers (initially greater then $90 \%$ ) has started to deteriorate at a rate of $0.5-1 \% /$ month. In order to solve some of the inefficiency problems, an extensive improvement program has been developed. The forward endcap was retrofitted with new improved RPCs in 2002, their efficiency has not significatly decreased since then. In the barrel, the RPCs have been replaced in 2004 and 2006 by 12 layers of limited streamer tube (LST) detectors and 6 layers of brass have been added to improve hadron absorption. The tubes have performed well since their installation with an efficiency of all layers at the geometrically expected level of $90 \%$. The pion rejection 


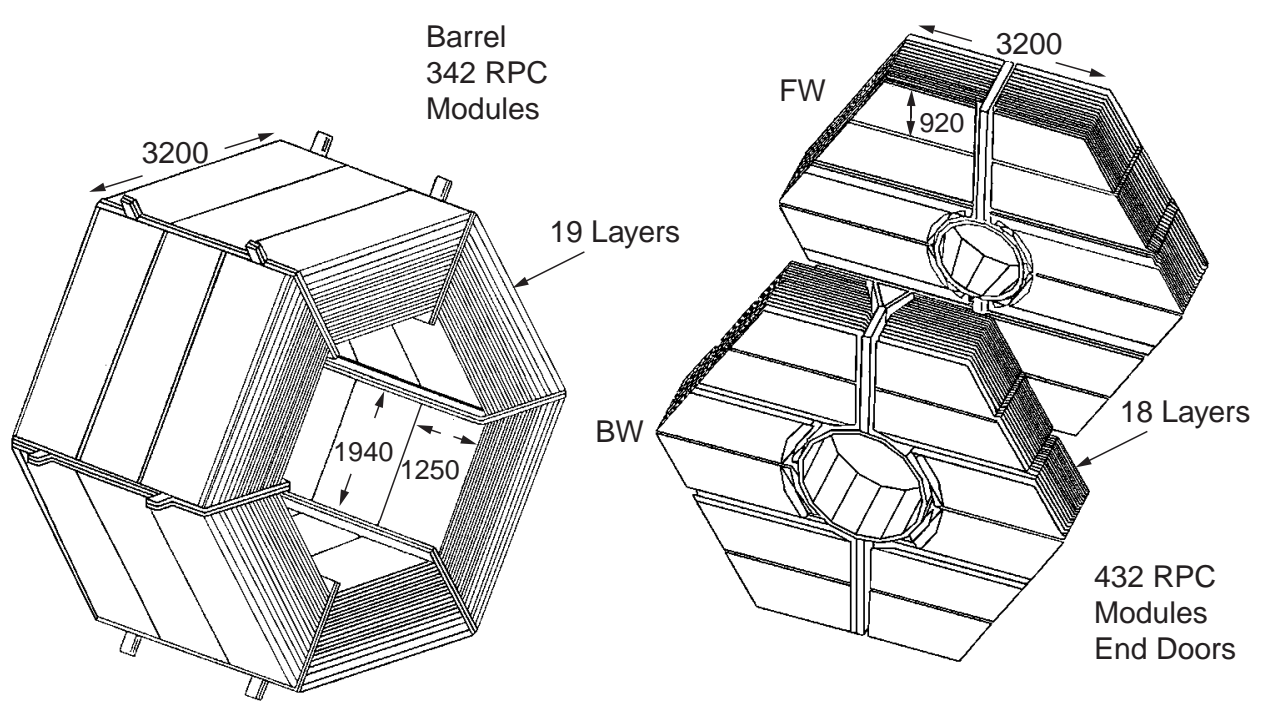

Figure 2.19: Overview of the IFR Barrel sectors and forward and backward end-doors; the shape of the RPC modules and the way they are stratified is shown.

versus muon efficiency is shown in Figure 2.20 for the LSTs and RPCs. The LSTs efficiency is better than the efficiency that the RPCs had, even during the Run1.
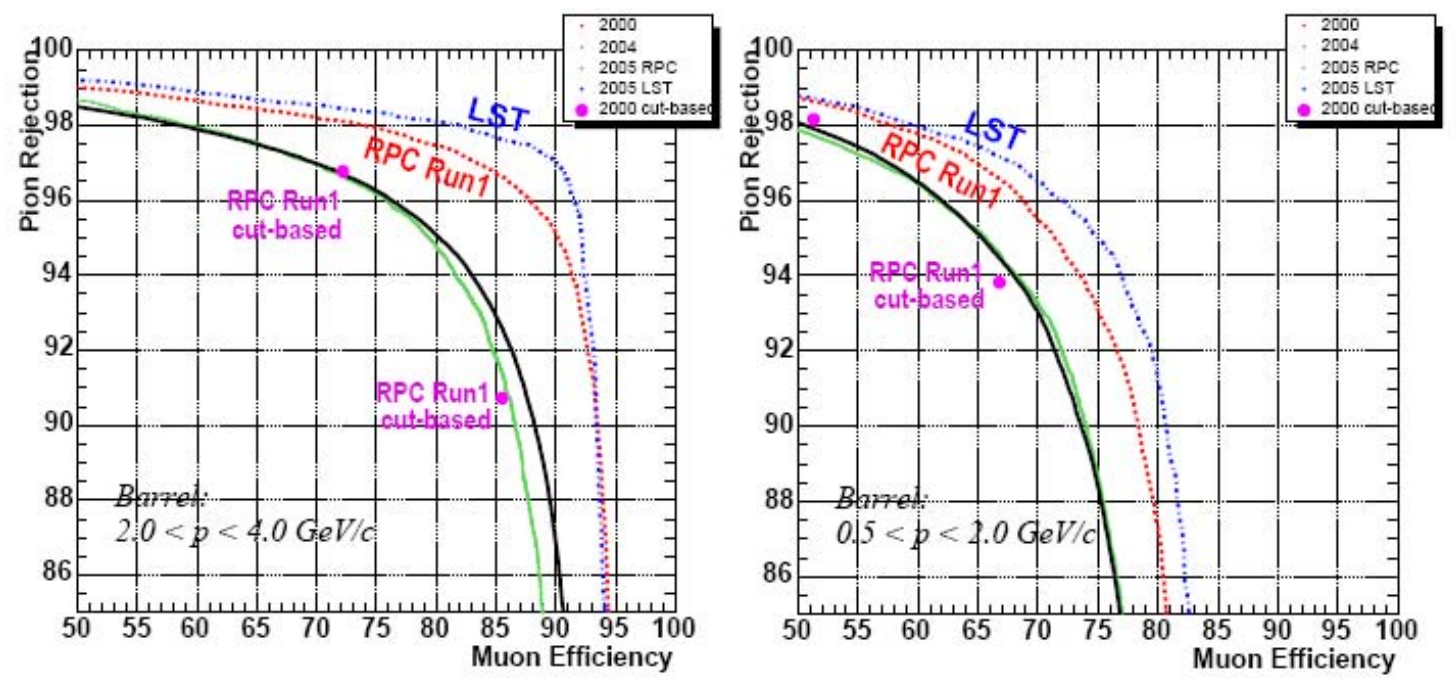

Figure 2.20: Pion rejection versus muon efficiency for two different momentum ranges (left: $2<p<4 \mathrm{GeV} / \mathrm{c}$, right $0.5<p<2 \mathrm{GeV} / \mathrm{c}$. The LST efficiency (blue) is compared with the RPC one for different Runs. We see the deterioration of the RPC performance between 2000 (red) and 2005 (green). 


\begin{tabular}{|l|r|r|r|r|r|r|}
\hline L3 Trigger & $\epsilon_{b \bar{b}}$ & $\epsilon_{B \rightarrow \pi^{0} \pi^{0}}$ & $\epsilon_{B \rightarrow \tau \nu}$ & $\epsilon_{c \bar{c}}$ & $\epsilon_{u d s}$ & $\epsilon_{\tau \tau}$ \\
\hline \hline Combined DCH filters & 99.4 & 89.1 & 96.6 & 97.1 & 95.4 & 95.5 \\
\hline Combined EMC filters & 93.5 & 95.7 & 62.3 & 87.4 & 85.6 & 46.3 \\
\hline Combined DCH+EMC filters & $>99.9$ & 99.3 & 98.1 & 99.0 & 97.6 & 97.3 \\
\hline Combined L1+L3 & $>99.9$ & 99.1 & 97.8 & 98.9 & 95.8 & 92.0 \\
\hline
\end{tabular}

Table 2.3: L3 trigger efficiency (\%) for various physics processes, derived from Monte Carlo simulation.

\subsubsection{Trigger}

The $B A B A R$ trigger is designed to select a large variety of physics processes (efficiency greater than $99 \%$ for $B \bar{B}$ events) while keeping the output rate below $400 \mathrm{~Hz}$ to satisfy computing limitations of the offline processing farms (beam induced background rates with at least one track with $p_{t}>120 \mathrm{MeV} / \mathrm{c}$ or at least one EMC cluster with $E>100 \mathrm{MeV}$ are typically 20 $\mathrm{kHz}$ ). The trigger accepts also $95 \%$ of continuum hadronic events and more than $90 \%$ of $\tau^{+} \tau^{-}$events. It is implemented as a two level hierarchy, the hardware Level 1 (L1) followed by the software Level 3 (L3).

The L1 trigger has an output rate of the order of $1 \mathrm{kHz}$ to $3 \mathrm{kHz}$, depending on the luminosity and background conditions. It is based on charged tracks in the DCH above a preset transverse momentum, showers in the EMC, and track detected in the IFR. L3 operates by refining and augmenting the selection methods used in L1. Based on both the complete event and L1 trigger information, the L3 software algorithm selects events of interest allowing them to be transferred to mass storage data for further analysis. It uses an algorithm based on the drift chamber tracking, which rejects beam-induced charged particle background produced in the material close to the IP, and a second algorithm based on the calorimeter clustering. Then, based on the L3 tracks and clusters, a variety of filters perform event classification and background reduction. Table 2.3 shows the L3 and L1+L3 trigger efficiency for some relevant physics processes, derived from MC simulation. One can see that the selection efficiency for $e^{+} e^{-} \rightarrow c \bar{c}$ events is close to $99 \%$.

\subsection{Particle identification}

After having described the different components of the detector, we will explain more specifically what are the informations used in the reconstruction of the $D_{s}^{+} \rightarrow K^{+} K^{-} e^{+} \nu_{e}$ channel. In this analysis, we have to reconstruct two charged kaons and one electron, which required the explicit identification of these particles. As the neutrino is not measured, we use the 
charged and neutral particles produced in the rest of the event to evaluate the missing energy and the $D_{s}^{+}$direction.

\subsubsection{Charged particle reconstruction}

The charged particle tracks are reconstructed by processing information from both tracking systems, the SVT and the DCH. Charged tracks are defined by five parameters shown in Figure $2.21\left(d_{0}, \phi_{0}, \omega, z_{0}, \tan \lambda\right)$ and their associated error matrix, measured at the point of closest approach to the z-axis. $d_{0}$ and $z_{0}$ are the distances between the point and the origin of the coordinate system in the $\mathrm{x}-\mathrm{y}$ plane and along the z-axis respectively. The angle $\phi_{0}$ is the azimuth of the track, $\lambda$ is the angle between the transverse plane and the track tangent vector at the point of closest approach and the $x$-axis, and $\omega=1 / p_{t}$ is the curvature of the track. $d_{0}$ and $\omega$ are signed variables and their sign depends on the charge of the track. The track finding and the fitting procedures use the Kalman filter algorithm [57] that takes into account the detailed distribution of material in the detector and the full magnetic field map.

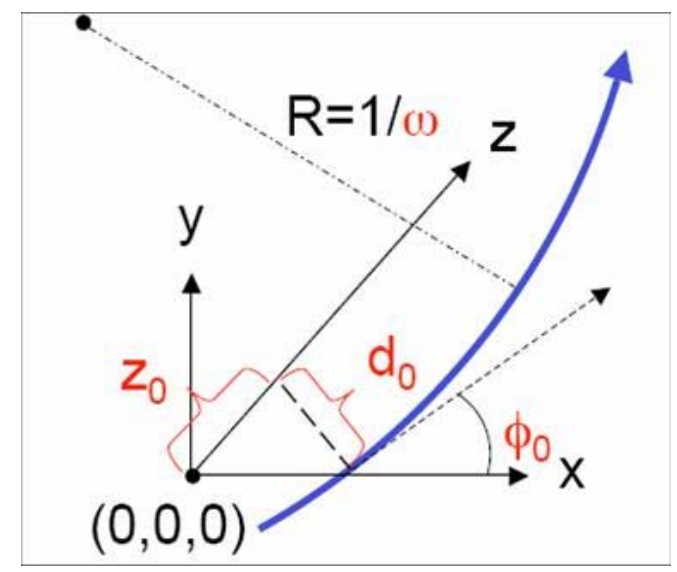

Figure 2.21: Definition of the parameters $d_{0}, \phi_{0}, \omega$ and $z_{0}$ used to define charged tracks.

The identified tracks are then organized in different categories depending on requirements on specific quantities. In the study of the $D_{s}^{+} \rightarrow K^{+} K^{-} e^{+} \nu_{e}$ decay channel, we use the list GoodTrackVeryLoose which is composed of charged tracks with momentum below $10 \mathrm{GeV} / \mathrm{c}$ (to remove tracks not compatible with the beam energy), distance of closest approach to the beam spot in the $\mathrm{x}-\mathrm{y}$ plane below $1.5 \mathrm{~cm}$, and below $10 \mathrm{~cm}$ along the $\mathrm{z}$ axis. 


\subsubsection{Neutral particles reconstruction}

Neutral particles (photons, $\pi^{0}$, neutral hadrons) are detected in the EMC as clusters of close crystals where energy has been deposited. They are required not to be matched to any charged track extrapolated from the tracking volume to the inner surface of the EMC. The lateral distribution of energy within a cluster depends heavily of the nature of the incident particle. The lateral moment, $L A T$, is defined as :

$$
L A T=\frac{\sum_{i=3}^{N} E_{i} r_{i}^{2}}{\sum_{i=3}^{N} E_{i} r_{i}^{2}+E_{1} r_{0}^{2}+E_{2} r_{0}^{2}},
$$

where $N$ is the number of crystals associated with the electromagnetic shower, $r_{0}$ is the average distance between two crystals, which is approximately $5 \mathrm{~cm}$ for the BABAR calorimeter, $E_{i}$ is the energy deposited in the i-th crystal, numbering them such that $E_{1}>E_{2}>\ldots>E_{N}$ and $r_{i}, \phi_{i}$ are the polar coordinates in the plane perpendicular to the line pointing from the interaction point to the shower cluster centroid. Considering that the summations start from $i=3$, they omit the two crystals containing the highest amounts of energy. Since electrons and photons deposit most of their energy in two or three crystals, the value of LAT is small for electromagnetic showers. Multiplying the energies by the squared distances enhances the effect for hadronic showers, compared with electromagnetic ones.

In this analysis, neutral particles are required to have an energy greater than $100 \mathrm{MeV}$ and a lateral moment smaller than 0.8 .

\subsubsection{Particle identification}

The particle identification is done by several algorithms with different levels of purity and efficiency. They allow the identification of stable particles: $e^{ \pm}, \mu^{ \pm}, \pi^{ \pm}, K^{ \pm}, p^{ \pm}$and $K_{L}^{0}$.

The identification of electrons is done using information predominantly from the electromagnetic calorimeter, but also coming from the DIRC and $d E / d x$ in the tracking devices at low momenta. In this analysis, for the electron identification, we use an algorithm called PidLHElectrons based on a likelihood [59]. It uses the following variables:

- For the EMC: the ratio $E / p$ of the energy deposited in the EMC and the momentum in the laboratory frame; $L A T$, the lateral shape of the calorimeter deposit; $\Delta \Phi$, a quantity characterizing the longitudinal energy distribution; and the number of crystals in the EMC cluster. 
- For the DIRC: the Cherenkov angle and the number of measured photons in the DIRC.

- For the DCH: the specific energy loss $d E / d x$.

The efficiency of the likelihood-based electron identification algorithm is shown in Figure 2.22 as function of the momentum for three different polar angle regions: the forward endcap, the forward barrel and the backward barrel [58]. The rate of pions and kaons misidentified as electrons is shown in Figure 2.23. This algorithm allows the selection of more than $90 \%$ of electron with momentum above $500 \mathrm{MeV}$, while the rate of misidentified hadrons is below $0.3 \%$ and $0.5 \%$ for pions and kaons with momentum above $1 \mathrm{GeV} / \mathrm{c}$, respectively.

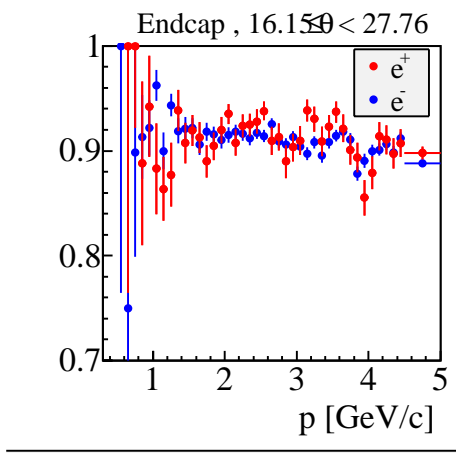

Selector : PidLHElectronSelector

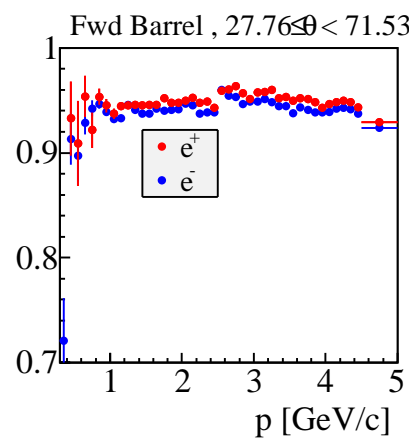

Dataset : run4-r18a

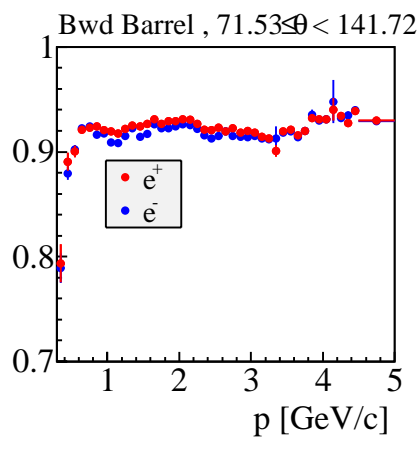

Tables created on 14/3/2006 (Data) $15 / 3 / 2006(\mathrm{MC})$

Figure 2.22: Electron identification efficiency for the likelihood-based electron selector as function of momentum, for three different angular regions.

The kaon selection is based on the information from the SVT, the DCH and the DIRC. For charged particles, a likelihood is computed as a product of three terms, one for each subdetector, and the selection is done using cuts on the relevant likelihood ratios. For example, the KLHTight selects kaons if $L_{K} /\left(L_{K}+L_{\pi}\right)>0.9$ and $L_{K} /\left(L_{K}+L_{p}\right)>0.2$ where the $L_{H}$ are the hadron likelihoods. The efficiency of kaons and protons as funtion of their momentum are shown in Figures 2.24 and 2.25 respectively.

In the present analysis, the data/MC agreement for tracking efficiency and particle identification has been studied and corrected using standard tools developped in BABAR. To correct neutral particle efficiencies, the central value and resolution of the measured energy are corrected. These corrections depend on the run period and are relatively small. For the PID, we correct for the data/MC efficiency differences in the following way. For a given particle selector, and using the PID efficiency tables derived from data and MC, the PID correction algorithm:

- rejects an accepted track with the probability $\epsilon_{\text {data }} / \epsilon_{M C}$ if $\epsilon_{\text {data }}<\epsilon_{M C}$, 


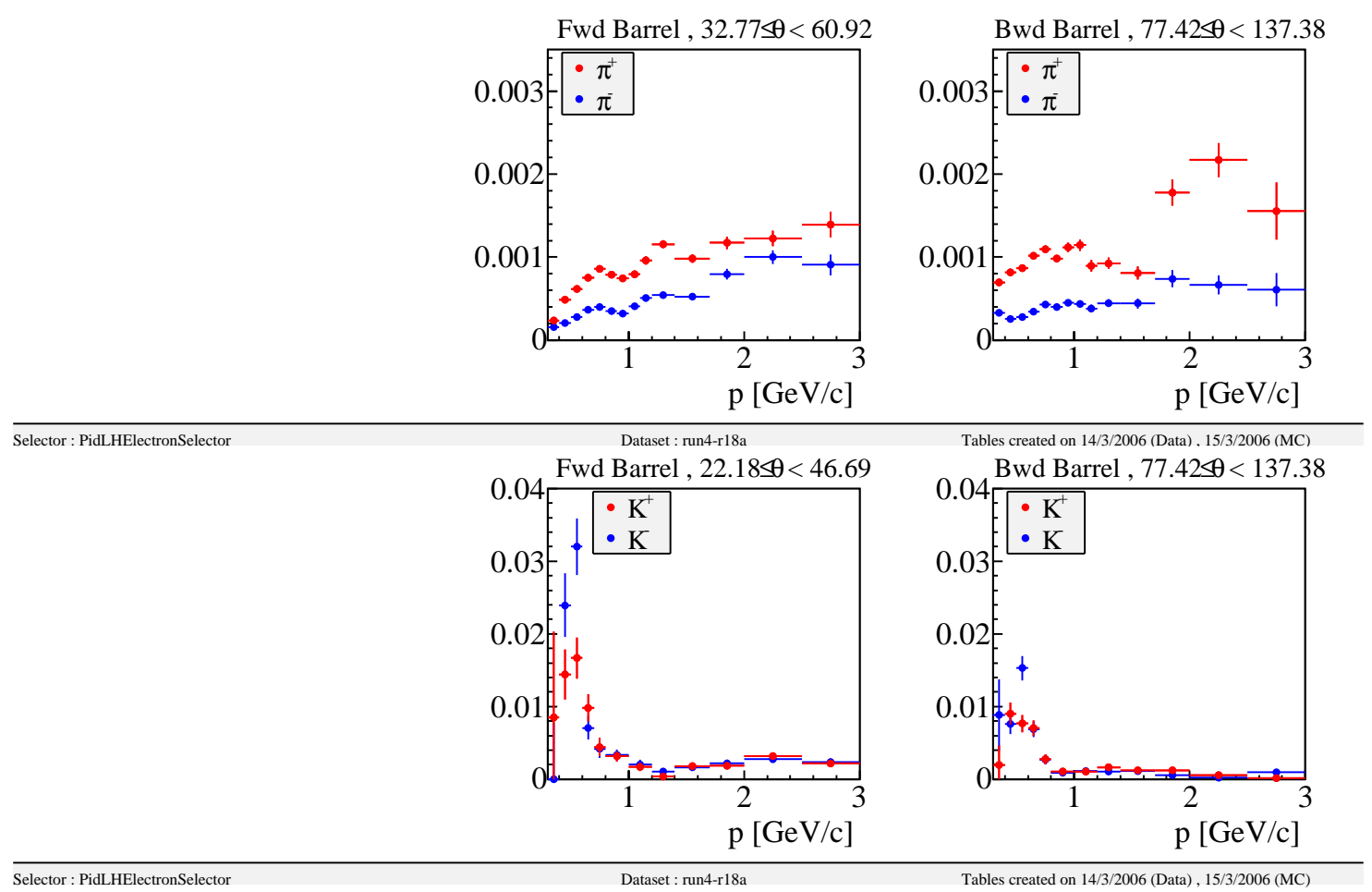

Figure 2.23: Hadron misidentification probability for the likelihood-based electron selector in two barrel regions.
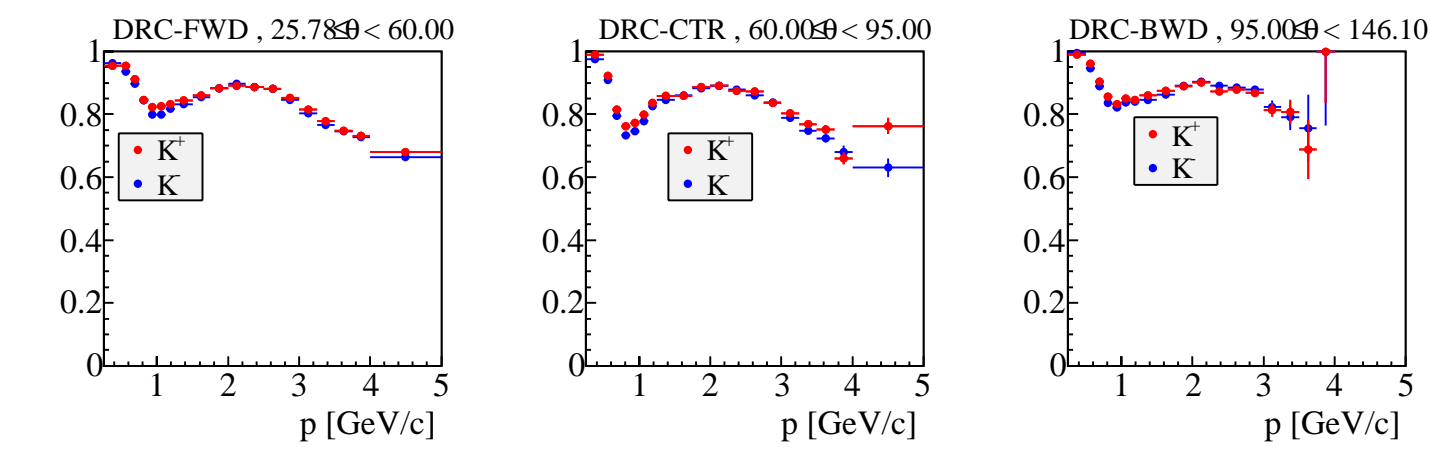

Selector : TightLHKaonMicroSelection

Dataset : run4-r18a

Tables created on 14/3/2006 (Data) , 15/3/2006 (MC)

Figure 2.24: Kaons identification efficiency for the likelihood-based kaons selector as function of momentum, for three different angular regions.

- accepts a rejected track with the probability $\left(\epsilon_{d a t a}-\epsilon_{M C}\right) /\left(1-\epsilon_{M C}\right)$ if $\epsilon_{d a t a}>\epsilon_{M C}$,

where $\epsilon_{\text {data }(M C)}$ is the data (MC) efficiency of the given selector. This procedure is applied to the main selectors of the analysis (kaon and electron selectors). 

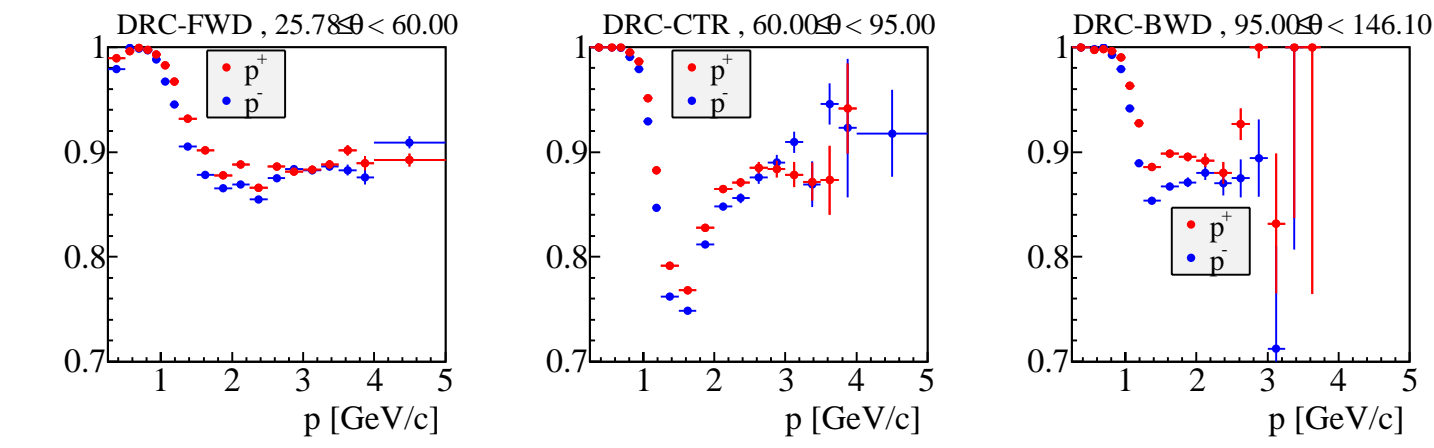

Selector : TightLHProtonSelection

Dataset : run4-r18a

Tables created on 14/3/2006 (Data) , 15/3/2006 (MC)

Figure 2.25: Protons identification efficiency for the likelihood-based protons selector as function of momentum, for three different angular regions. 


\section{Chapter 3}

\section{Event reconstruction and background rejection}

'Curiouser and curiouser!' cried Alice (she was so much surprised, that for the moment she quite forgot how to speak good English); 'now I'm opening out like the largest telescope that ever was! Good-bye, feet!'

In this chapter, after a brief overview of the analysis method and data sample, we detail the different steps of the $D_{s}^{+} \rightarrow \phi e^{+} \nu_{e}$ candidate's selection and reconstruction. This gives access to the kinematic variables of the decay used in the extraction of the form factors parameters. Then we explain the background rejection and its final composition as obtained from the simulation, and we discuss the characteristics of the reconstruction methods such as the resolution and efficiency for the kinematic variables.

\subsection{Analysis method and data sample}

\subsubsection{Analysis method}

This analysis is based on the reconstruction of $D_{s}^{+}$mesons produced in continuum $c \bar{c}$ events and which decay semileptonically. $D_{s}^{+}$from $B$ decays are not used as one expects poorer resolution on the reconstruction of the missing neutrino and less separation between $D_{s}^{+}$ decay products and the other particles present in the event. Only final states with electrons are considered for the following reasons: 
- the analysis is not statistically limited;

- electrons are better identified than muons;

- the measurement is simpler as the contribution of one hadronic form factor, which is proportionnal to the lepton mass squared, can be neglected.

At the energy of the $\Upsilon(4 S)$, the fragmentation of the $c$ and $\bar{c}$ quarks leads to two chadrons emitted mostly in opposite directions in the center-of-mass frame. To reconstruct the direction of the two jets, we use the event thrust axis, which is the direction maximizing the sum of the projections of the particles momenta along this direction. The event thrust axis is determined from all charged and neutral particles measured in the center-of-mass system and a plane perpendicular to this axis is used to define two hemispheres, each containing one of the two jets produced by quark fragmentation. In each hemisphere, we search for decay products of the $D_{s}^{+}$: a charged lepton and two oppositely charged kaons. Figure 3.1 gives an illustration of the topology of a signal event.

Since the neutrino $\left(\nu_{e}\right)$ momentum is unmeasured, a kinematic fit is performed, constraining the invariant mass of the candidate $\left(e^{+} K^{+} K^{-} \nu_{e}\right)$ system to the $D_{s}^{+}$mass. In this fit, the $D_{s}^{+}$momentum and the neutrino energy are estimated from the other particles measured in the event. This method is different form the one used by the Belle collaboration, to study $D^{0}$ semileptonic decays [60], in which events are kept if the only missing particle is the neutrino. They obtain a good resolution on kinematic variables at the price of a very low efficiency. They have not yet published a $D_{s}^{+}$semileptonic signal with their approach.

After having rejected the background, using mainly standard cuts and two Fisher discriminant variables (one to decrease $B \bar{B}$ background events and the second one to decrease $c \bar{c}$ background events), the form factors parameters are extracted with a binned maximum likelihood fit to the four dimensional decay distribution $\left(q^{2}, \theta_{e}, \theta_{V}, \chi\right)$. In this fit, a dedicated sample of signal MC is weighted according to the form factor model and the background is estimated from the generic MC corrected to agree with data. This is why the most crucial part of this analysis relies on the detailed studies and adjustements of the simulation of signal and backgrounds. This is done using several control data samples.

\subsubsection{Data sample}

Results have been obtained with Run 1 to 4 on-resonance data, corresponding to $214 \mathrm{fb}^{-1}$ integrated luminosity. In addition, generic MC samples of charm, b-hadrons and light quark pairs $(u \bar{u}, d \bar{d}, s \bar{s})$, as well as a MC sample of $D_{s}^{+} \rightarrow \phi e^{+} \nu_{e}$ signal, have been used. The analyzed MC statistics are given in Table 3.1. Off-peak events, which are recorded $40 \mathrm{MeV}$ 


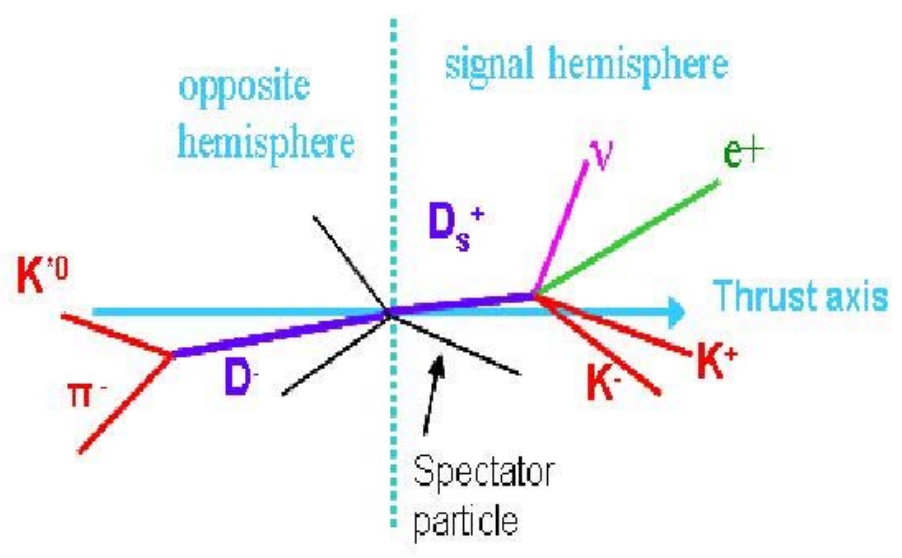

Figure 3.1: Example of a signal event produced in a $\Upsilon(4 S) \rightarrow c \bar{c}$ decay, for which the event topology is "jet-like". There are two hemispheres defined by the plane perpendicular to the thrust axis, each containing usually a charm hadron.

below the $\Upsilon(4 S)$, do not contain $B$ mesons. They are not used in the fit because the sample is much smaller than the on-resonance data sample. Nevertheless, they are used to check the data/MC agreement of the $B \bar{B}$ background component.

\begin{tabular}{|c|c|c|}
\hline $\begin{array}{c}\text { Event } \\
\text { sample }\end{array}$ & $\begin{array}{c}\text { analyzed } \\
\text { statistics } \\
\text { (million events) }\end{array}$ & $\begin{array}{c}\text { expected } \\
\text { cross section } \\
\text { (nb) }\end{array}$ \\
\hline$B^{+}-B^{-}$ & 387.97 & 0.525 \\
\hline $\mathrm{B}_{\mathrm{d}}^{0}-\overline{\mathrm{B}_{\mathrm{d}}^{0}}$ & 389.57 & 0.525 \\
\hline$u \bar{u}, d d, s \bar{s}$ & 498.23 & 2.09 \\
\hline$c \bar{c}$ & 480.78 & 1.3 \\
\hline$D_{s}^{+} \rightarrow \phi e^{+} \nu_{e}$ & 12.6 & $3.2510^{-3}$ \\
\hline
\end{tabular}

Table 3.1: Analyzed statistics of simulated events and expected corresponding cross sections. For the signal, we give the expected cross section multiplied by the branching fraction.

\subsection{Event selection}

\subsubsection{Skim preselection}

The first selection step is done by a tool called CharmSL skim. It allows the reduction of the total data sample to a smaller sample enriched in charm semileptonic decays from continuum $c \bar{c}$ events. The cuts used are the following: 
- BGFMultiHadron filter, which selects hadronic events;

- at least one electron with a momentum in the center of mass frame $P_{l}^{*}$ greater than $0.5 \mathrm{GeV} / \mathrm{c}$;

- $R_{2}>0.2$, where $R_{2}$ is the normalized second Fox-Wolfram moment (H2/H0) [61]. This cut rejects a large fraction of $B$ events which have a more spherical topology with respect to charm events;

- $M_{o p p}>0.5 \mathrm{GeV} / \mathrm{c}^{2}$, where $M_{o p p}$ is the mass of the system formed by all particles in the hemisphere opposite to the lepton. This cut rejects lepton pairs and two-photons events;

- $M_{t a g}>0.13 \mathrm{GeV} / \mathrm{c}^{2}$, where $M_{t a g}$ is the mass of the system formed by the lepton and the leading particle in the lepton hemisphere. This cut removes events where the lepton is the only particle in the hemisphere.

The skim rejects about $98 \%$ of the on-resonance data while keeping $36 \%$ of the signal.

\subsubsection{Global selection criteria}

The following lists of particles, defined in section 2.3, have been used:

- for electrons: PidLHElectrons;

- for kaons: KLHTight;

- for protons: pLHTight;

- for charged tracks: GoodTracksVeryLoose;

- for neutrals: GoodPhotonDefault.

It has been checked that electron, kaon and proton candidates are included in the charged track list. A priority has been applied on identified particles to avoid double counting: electron $>$ kaon $>$ proton $>$ pion ${ }^{1}$. Masses have been attributed to charged particles according to the result of the particle identification (PID). Unidentified charged particles are assumed to be pions and all neutrals are assumed to be photons. The direction of the event thrust axis is taken in the interval $\left|\cos \left(\theta_{\text {thrust }}\right)\right|<0.6$ to minimize the loss of particles close to the beam axis. Figure 3.2, which shows the $D_{s}^{+}$direction versus the event thrust

\footnotetext{
${ }^{1}$ For instance, if a particle is present in the electron and in the kaon lists, it is assumed to be an electron.
} 
direction for $D_{s}^{+} \rightarrow \phi \pi^{+}$data events, illustrates this effect: if the detector would have been symmetric, the repartition of events in the upper branch would have been the same as in the lower branch. But we observe a loss of events in the upper branch, when $\left|\cos \left(\theta_{\text {thrust }}\right)\right|>0.6$ and $\cos \left(\theta_{D_{s}}\right)>0$, which corresponds to the forward direction.

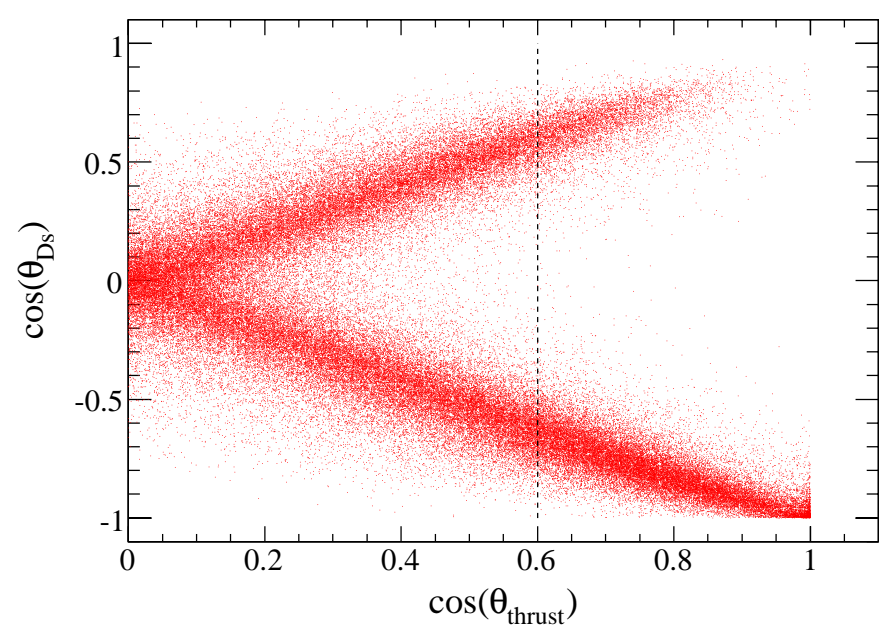

Figure 3.2: Cosine of $\theta_{D_{s}}$ versus cosine of the thrust axis for $D_{s}^{+} \rightarrow \phi \pi^{+}$data events.

\subsection{Event reconstruction}

Charged and neutral particles are boosted to the center-of-mass system and the event thrust axis is determined. A plane perpendicular to the thrust axis is used to define two event hemispheres. By definition, the momentum projection along the thrust direction of particles belonging to one hemisphere are all positive or all negative. Each hemisphere is considered in turn.

We select as a candidate signal event a positron and two charged kaons, in the same hemisphere. A vertex fit is performed and the candidate is kept if the $\chi^{2}$ probability of the fit is larger than $10^{-3}$.

To estimate the neutrino $\left(\nu_{e}\right)$ momentum, a constrained fit, imposing the $D_{s}^{+}$mass to the $\left(e^{+} K^{+} K^{-} \nu_{e}\right)$ system, is applied. This fit uses, as input, estimates of the $D_{s}^{+}$direction and of the neutrino energy obtained from all particles in the event:

- the $D_{s}^{+}$direction estimate is taken as the direction of the vector opposite to the sum of the momentum of all reconstructed particles except the two kaons and the charged lepton; 
- the neutrino energy is evaluated by subtracting from the hemisphere energy, the energy of reconstructed particles contained in that hemisphere. The energy of each hemisphere has been evaluated by considering that the total center-of-mass energy is shared by two objects of mass corresponding to the measured hemisphere masses ${ }^{2}$. As a $D_{s}^{+}$ is expected to be present in the analyzed hemisphere and as at least a $D$ meson is produced in the opposite hemisphere, minimum values for hemisphere masses have been imposed. For an hemisphere $i$, the energy $E_{h e m}^{(i)}$ and mass $m_{h e m}^{(i)}$ can be written as:

$$
\begin{gathered}
E_{\text {hem }}^{(i)}=1 / 2\left(\sqrt{s}+\frac{m_{\text {hem }}^{(i) 2}-m_{\text {hem }}^{(j) 2}}{\sqrt{s}}\right) \\
m_{\text {hem }}^{(i)}=\max \left(m_{\text {hem }}^{(i)}(\text { measured }), m_{D}\right)
\end{gathered}
$$

Errors on the neutrino energy and $D_{s}^{+}$direction are evaluated as function of the missing energy in the opposite hemisphere (see section 4.1.2). Keeping events with a $\chi^{2}$ probability larger than $10^{-3}$ in the mass constrained fit, the $D_{s}^{+} 4$-momentum is obtained and used in the computation of the other kinematic variables $\left(q^{2}, \cos \theta_{V}, \cos \theta_{e}, \chi\right)$. Events with a non-physical value of $q^{2}$ (negative) are rejected as they correspond mainly to background. The fraction of signal events with a negative $q^{2}$ is about $0.2 \%$.

Other particles present in the hemisphere, which are not decay products of the $D_{s}^{+}$candidate are named "spectator" particles. They originate from the beam interaction point and are emitted during hadronisation of the created $c$ and $\bar{c}$ quarks. In the following, the "leading" track is defined as the spectator track having the largest momentum. Information from the spectator system is used to decrease the contribution from the combinatorial background. As charm hadrons take a large fraction of the charm quark energy, charm decay products have higher average energies than spectator particles. To study $D_{s}^{+} \rightarrow \phi e^{+} \nu_{e}$, we select events with a $K^{+} K^{-}$mass between $1.01 \mathrm{GeV} / \mathrm{c}^{2}$ and $1.03 \mathrm{GeV} / \mathrm{c}^{2}$.

\subsection{Background rejection}

Two Fisher discriminant variables are used to reject $B \bar{B}$ and $c \bar{c}$ events. The present analysis does not depend on exact cut values on the two discriminant variables. It has been verified that results are stable over a reasonable variation range for the selection criteria.

\footnotetext{
${ }^{2}$ The hemisphere mass is the mass of the system corresponding to the sum of the 4-vectors for particles contained in that hemisphere.
} 


\subsubsection{Fisher analysis method}

Starting from a set of discriminating variables, the aim of the Fisher analysis is to compute the linear combination of these variables that gives a good separation between signal and background. To perform this analysis we have used the TMVA package [62]. We consider a sample of $n_{v a r}$ variables $x_{k}$, their mean values $\bar{x}_{k}$, the means of the corresponding signal and background samples (class-specific samples) $\bar{x}_{S(B), k}$, and the total covariance matrix $C$ of the sample. The covariance matrix can be decomposed into the sum of a within-class matrix $(W)$ and a between-class matrix $(B)$. They respectively describe the dispersion of events relative to the means of their own class, and relative to the overall sample means:

$$
\begin{aligned}
W_{k l} & =\sum_{U=S, B}\left\langle x_{U, k}-\bar{x}_{U, k}\right\rangle\left\langle x_{U, l}-\bar{x}_{U, l}\right\rangle \\
B_{k l} & =\frac{1}{2} \sum_{U=S, B}\left(\bar{x}_{U, k}-\bar{x}_{k}\right)\left(\bar{x}_{U, l}-\bar{x}_{l}\right)
\end{aligned}
$$

The Fisher coefficients, $F_{k}$, are then given by:

$$
F_{k}=\frac{\sqrt{N_{S} N_{B}}}{N_{S}+N_{B}} \sum_{l=1}^{n_{v a r}} W_{k l}^{-1}\left(\bar{x}_{S, l}-\bar{x}_{B, l}\right)
$$

where $N_{S,(B)}$ are the number of signal (background) events in the sample. The Fisher discriminant $y(i)$ for event $i$ is given by

$$
y(i)=F_{0}+\sum_{k=1}^{n_{\text {var }}} F_{k} x_{k}(i) .
$$

The offset $F_{0}$ centers the sample mean $\bar{y}$ of all $N_{s}+N_{B}$ events at zero.

The discrimination power of a variable is given by the diagonal quantity $B_{k k} / C_{k k}$, which is used for the ranking of the input variables.

For this analysis, several methods have been tested. The Fisher approach has similar performances as the others and is the simplest to use. 

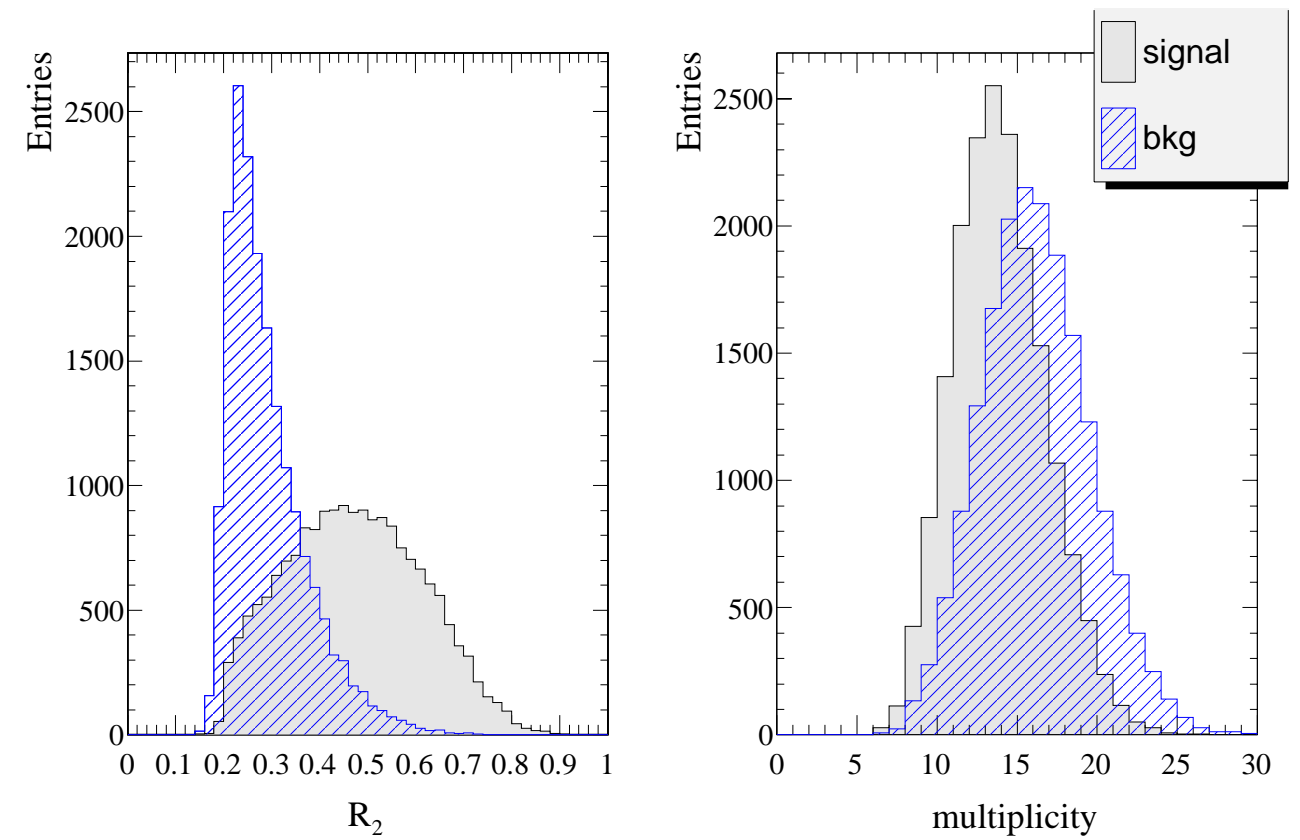

Figure 3.3: Distributions of the variables used in the Fisher discriminant analysis to decrease the $B \bar{B}$ event background. The left plot is the normalized second Fox-Wolfram moment $\left(R_{2}\right)$, the right plot is the total particle multiplicity. Below $R_{2}=0.2$, events have been cut by the skim selection.

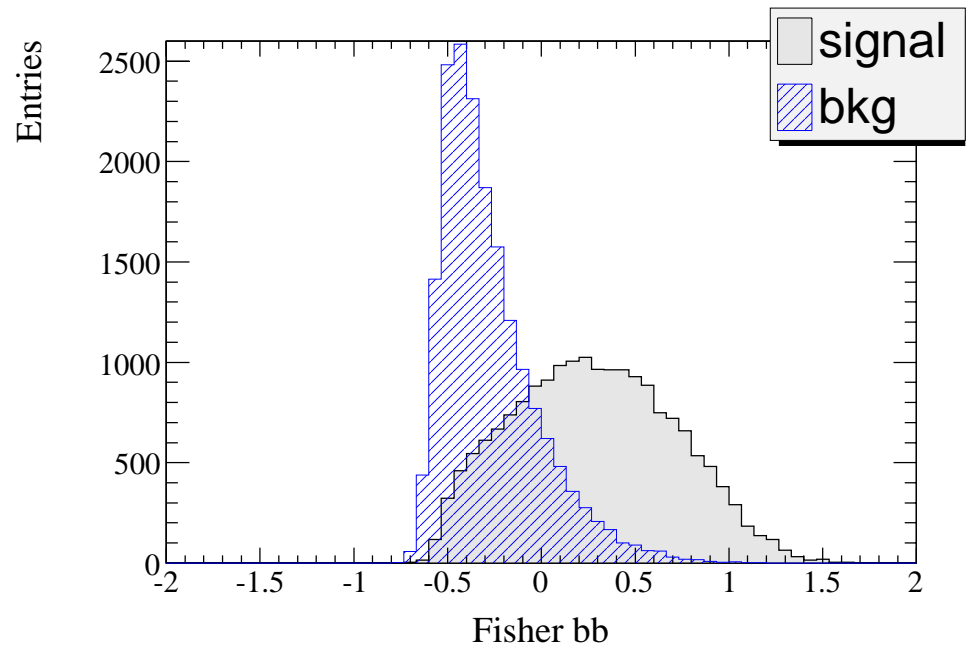

Figure 3.4: Distributions of the Fisher discriminant used to decrease the $B \bar{B}$ event background. 


\subsubsection{Rejection of $B \bar{B}$ events}

$B \bar{B}$ events are more spherical and have, on average, a larger particle multiplicity than $c \bar{c}$. The variables reflecting these characteristics are shown in Figure 3.3. They are combined linearly in a Fisher discriminant, shown on Figure 3.4, and events are selected requiring:

$$
F_{B B}=2.9297 * R_{2}-0.0112 * \text { mult }-0.9534>0 .
$$

For events selected with the previous cuts and the skim, the signal efficiency for this cut is $71 \%$. It removes $86 \%$ of background candidates from $B \bar{B}$ events.

\subsection{3 $c \bar{c}$ background rejection}

To reject $c \bar{c}$ background events, we use different variables describing the spectator system. The fraction of signal events without a spectator, i.e with no accompanying particle in the candidate hemisphere is $4 \%$ in data (measured using $D_{s} \rightarrow \phi \pi$ decays). These events are rejected as they would have required a dedicated Fisher analysis.

The following variables have been used to define a Fisher discriminant $F_{c c}$ (for each variable, the discrimination power, defined in section 3.4.1, is given within parentheses):

- the $D_{s}^{+}$momentum, $P_{D_{s}}$, after the constrained fit (0.2023);

- the mass of the spectator system, $m_{\text {spec. }}$, which has lower values for signal events (0.1432);

- the momentum of the leading spectator track, $P_{\text {leading }}(0.1276)$;

- the momentum of spectator system, $P_{\text {spec }}(0.1967)$.

Distributions of these variables for signal and $c \bar{c}$ background events are given in figure 3.5. One can note two peaks in the spectator system mass distribution for signal, at about 150 $\mathrm{MeV}$ and $500 \mathrm{MeV}$. They correspond to events where there is only one reconstructed particle in the spectator system: a pion or a kaon.

They are combined to form the discriminant (see Figure 3.6):

$$
F_{c c}=0.344 * P_{D_{s}}-0.375 * P_{\text {spec }}+0.038 * m_{\text {spec. }}+0.258 * P_{\text {leading }}-0.811
$$

Events have been kept for $F_{c c}>0$. For events selected with $F_{B B}>0$, this cut removes $72 \%$ of the remaining $c \bar{c}$ background and corresponds to a signal efficiency of $71 \%$. 

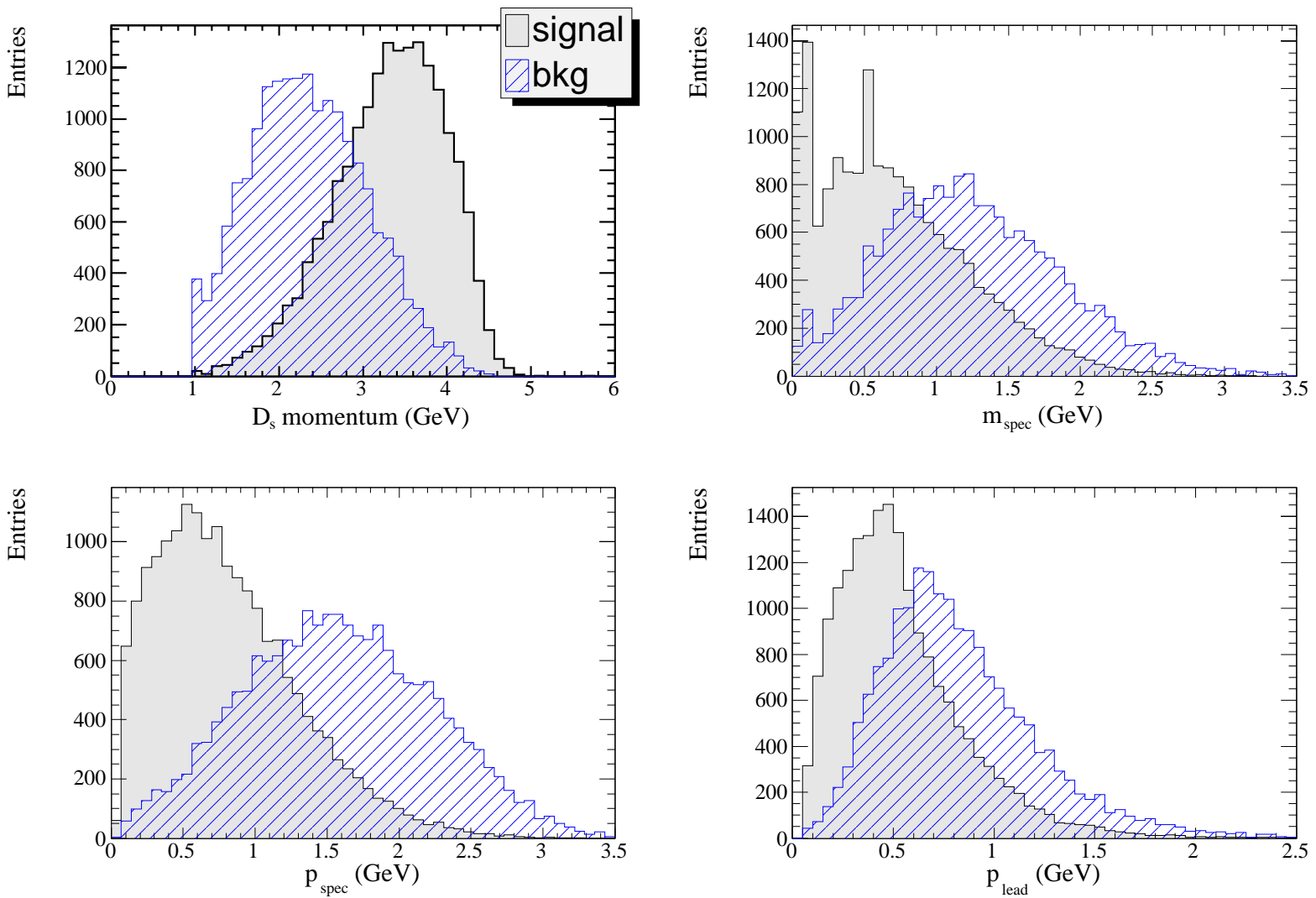

Figure 3.5: Distributions of variables used in the Fisher discriminant analysis to decrease the $c \bar{c}$ event background. From upper left, the variables are: the $D_{s}^{+}$momentum, the spectator system mass, the total spectator system momentum and the leading momentum of spectator tracks (all momenta are evaluated in the $e^{+} e^{-}$center-of-mass frame).

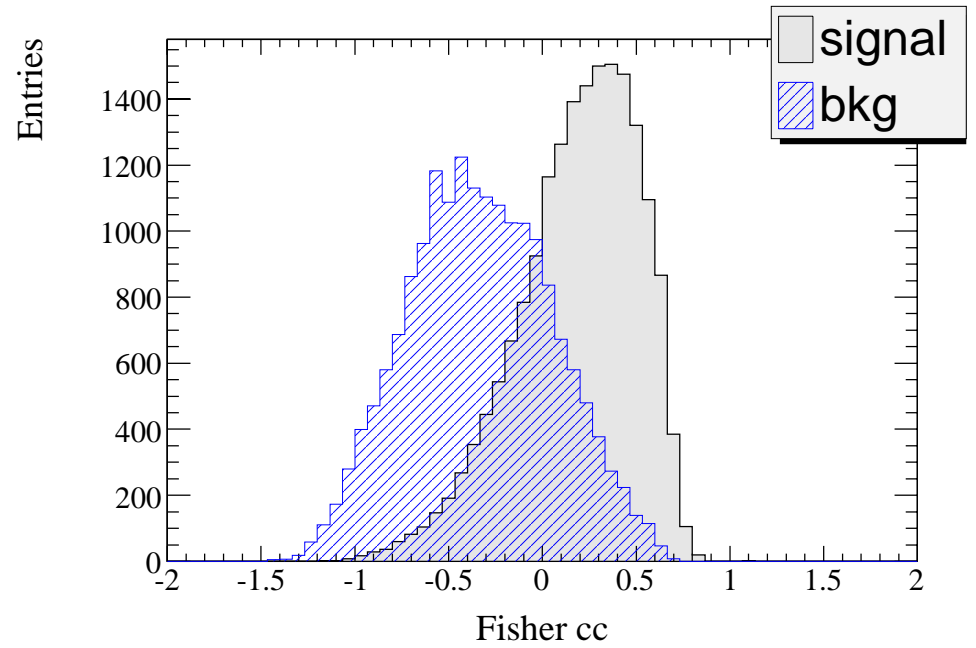

Figure 3.6: Distributions of the Fisher discriminant used to decrease the cc $\bar{c}$ event background. 


\subsubsection{Rejection of gamma conversion}

The last selection cut consists in rejecting converted photons which enter in about $30 \%$ of the peaking background (see below). To do this, we check that the candidate electron is not originating from converted photon candidates. This cut rejects about $50 \%$ of converted photons. The signal efficiency is $96 \%$.

\subsubsection{Background composition}

Within the selected $K^{+} K^{-}$mass interval (between $1.01 \mathrm{GeV} / \mathrm{c}^{2}$ and $1.03 \mathrm{GeV} / \mathrm{c}^{2}$ ), there are 31, 839 data events. From MC simulation, normalized to the data luminosity, the expected background fraction in selected events is $18 \%$. This background contains $12.5 \%$ light quark events (noted $u d s$ events), $42.5 \% B \bar{B}$ events and $45 \% c \bar{c}$ events. Using simulated events, these components are displayed in Figure 3.7. The ratio $\frac{S}{\sqrt{S+B}}$ is shown on Figure 3.8.

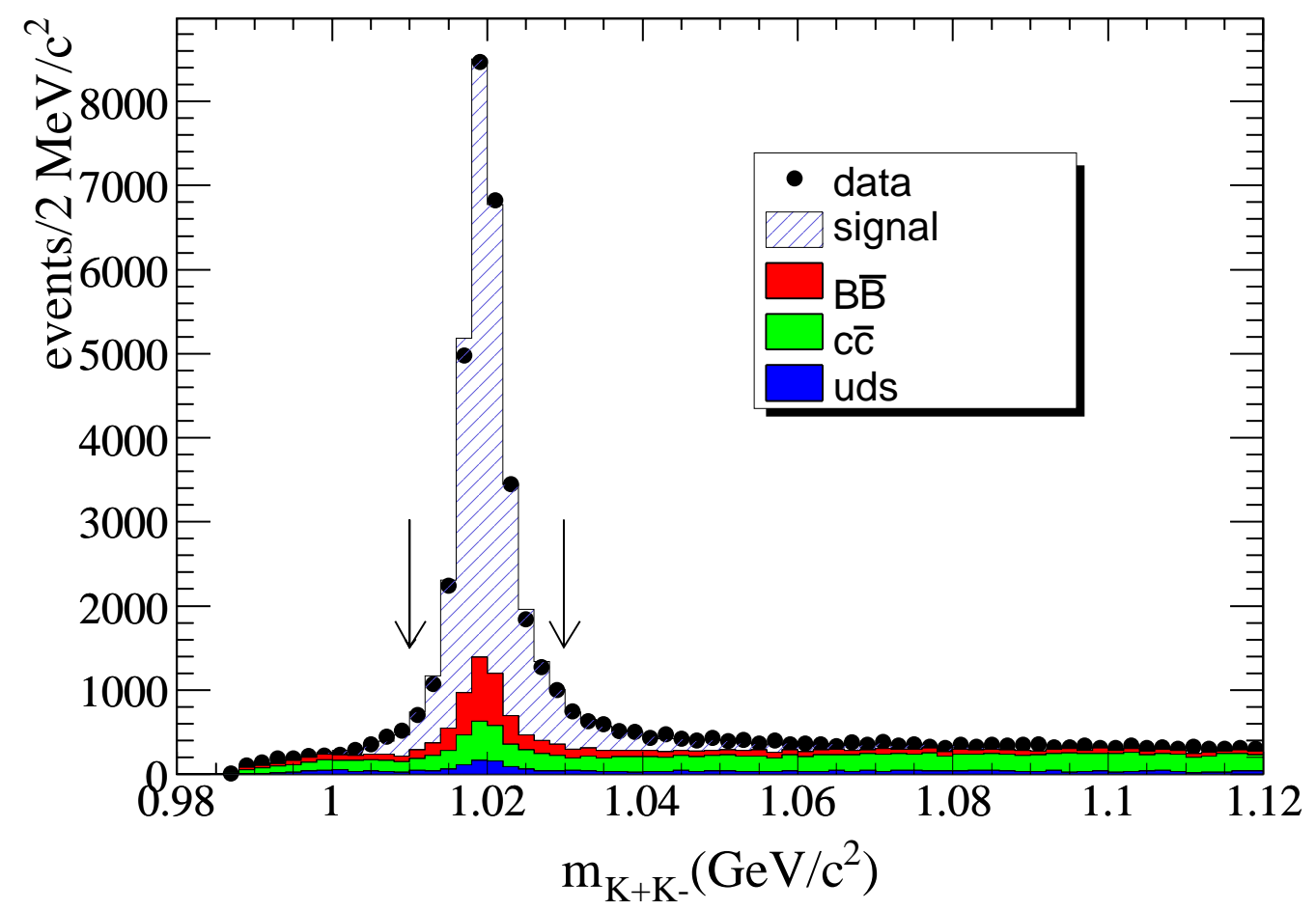

Figure 3.7: $K^{+} K^{-}$mass distribution from data and simulated events. MC events have been normalized to the data luminosity according to the production cross sections. The arrows indicate the selected mass region.

For each of the different background sources ( $u d s, B \bar{B}$ and $c \bar{c}$ events), there is a peaking and a non-peaking component. The peaking background (70\% of the total background) 


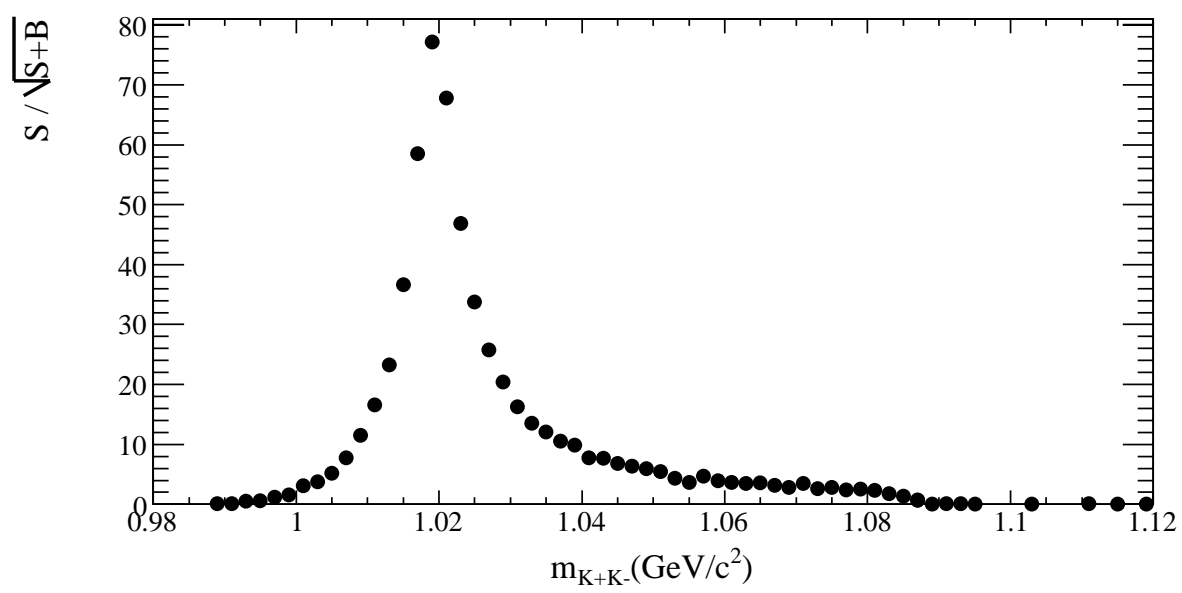

Figure 3.8: $\frac{S}{\sqrt{S+B}}$ as function of the $K^{+} K^{-}$mass.

corresponds to a real $\phi$ combined with a candidate electron. The latter may originate from a $B$-meson, a charm meson, from photon conversion or a $\pi^{0} / \eta$ Dalitz decay, or be a hadron misidentified as an electron.

$\phi$ mesons from background events are expected to originate from the primary vertex, from a charm meson, or a $B$-meson. These different sources are summarized in Table 3.2.

\begin{tabular}{|c|c|c|c|c|}
\hline $\begin{array}{c}\phi \text { and lepton } \\
\text { origin }\end{array}$ & $\begin{array}{c}\text { fake } \\
\text { lepton }\end{array}$ & $\begin{array}{c}\text { conversion } \\
\text { or } \pi^{0}\end{array}$ & $\begin{array}{c}\text { lepton from } \\
\text { charm }\end{array}$ & $\begin{array}{c}\text { lepton from } \\
\mathrm{B}\end{array}$ \\
\hline $\begin{array}{c}\phi \text { from } \\
\text { fragmentation }\end{array}$ & 6.5 & 6.4 & 16 & 0 \\
\hline $\begin{array}{c}\phi \\
\text { from } D_{s}^{+}\end{array}$ & 4.1 & 8.3 & 0.4 & 0 \\
\hline $\begin{array}{c}\phi \\
\text { from } D\end{array}$ & 1.7 & 4.9 & 0.5 & 0 \\
\hline $\begin{array}{c}\phi \\
\text { from } B\end{array}$ & 0.1 & 0.1 & 0 & 50.8 \\
\hline
\end{tabular}

Table 3.2: Peaking background composition (in \%) obtained from the generic Monte Carlo sample. The first column gives the $\phi$ origin and the first line the lepton origin. Hadrons reconstructed as leptons are named fake leptons. When the $\phi$ is measured in a $B \bar{B}$ event, we have not distinguished $\phi$ cascading from charm decays.

In the selected $K^{+} K^{-}$mass region, the non-peaking background, which represents $30 \%$ of the total background, contains $11 \%$ uds events, $24 \% B \bar{B}$ events and $65 \% c \bar{c}$ events. The composition of each background type has been studied. No dominant component has been found in $B \bar{B}$ events. For the $c \bar{c}$ non-peaking background, we find that the lepton originates 
from a charm meson in $94 \%$ of cases, from conversion or Dalitz decay in $2 \%$, and the rest are fake leptons. For the lepton coming from a charm meson, we have looked at the origin of the charged kaons. The most important components, considering only events with a candidate positron, are the following :

- $K^{+}$from fragmentation and $K^{-}$from a $D^{0}: 44 \%$

- $K^{-}$from fragmentation and $K^{+}$from a $D_{s}^{+}: 19 \%$

- $K^{+}$from fragmentation and $K^{-}$from a $D^{+}: 13 \%$

- $\mathrm{K}^{+}$from fragmentation and $\mathrm{K}^{-}$from fragmentation: $13 \%$

- one fake kaon and one kaon from a charm meson: $7 \%$

The first case corresponds mainly to a $D^{0} \rightarrow K^{-} e^{+} \nu_{e}$ decay, and the second is related to the signal. Dedicated measurements have been done to improve the MC simulation of these different components of the background using various data and MC samples. This is explained in Section 4.3.3.

\subsection{Measurement of decay characteristics and reconstruc- tion efficiency}

In this analysis, the form factors parameters of the $D_{s}^{+} \rightarrow \phi e^{+} \nu_{e}$ decay ( $q^{2}$ dependence and relative contributions of the three form factors) are obtained from the reconstructed values of the four kinematic variables $q^{2}\left(q_{r}^{2}\right), \cos \theta_{e}\left(\cos \left(\theta_{e}\right)_{r}\right), \cos \theta_{V}\left(\cos \left(\theta_{V}\right)_{r}\right)$ and $\chi\left(\chi_{r}\right)$ Using simulated events the reconstruction accuracy on these variables has been assessed by comparing the reconstructed and simulated values.

To illustrate the characteristics of the reconstruction method on simulated events, resolutions have been fitted to the sum of two Gaussian distributions, the corresponding standard deviations are listed in Table 3.3. These distributions are not used in the analysis which takes into account the migration of events in four dimensions as expected from the simulation.

The performance of the reconstruction and the data/MC agreement have been verified in the analysis of $D^{*+} \rightarrow D^{0} \pi^{+}, D^{0} \rightarrow K^{-} e^{+} \nu_{e}$ decays using $D^{*+} \rightarrow D^{0} \pi^{+}, D^{0} \rightarrow K^{-} \pi^{+} \pi^{0}$ decays [46]. After having reconstructed this channel, the $\pi^{0}$ is removed and the event is analyzed as if it was a $D^{0} \rightarrow K^{-} e^{+} \nu_{e}$ transition. To avoid trivial effects from kinematics the pion mass is assigned to the " $e^{+}$" and the $\pi^{0}$ mass is assigned to the " $\nu_{e}$ ". With these events it is possible to compare, using real events, the values of $q^{2}$ as given by the mass constrained fit 

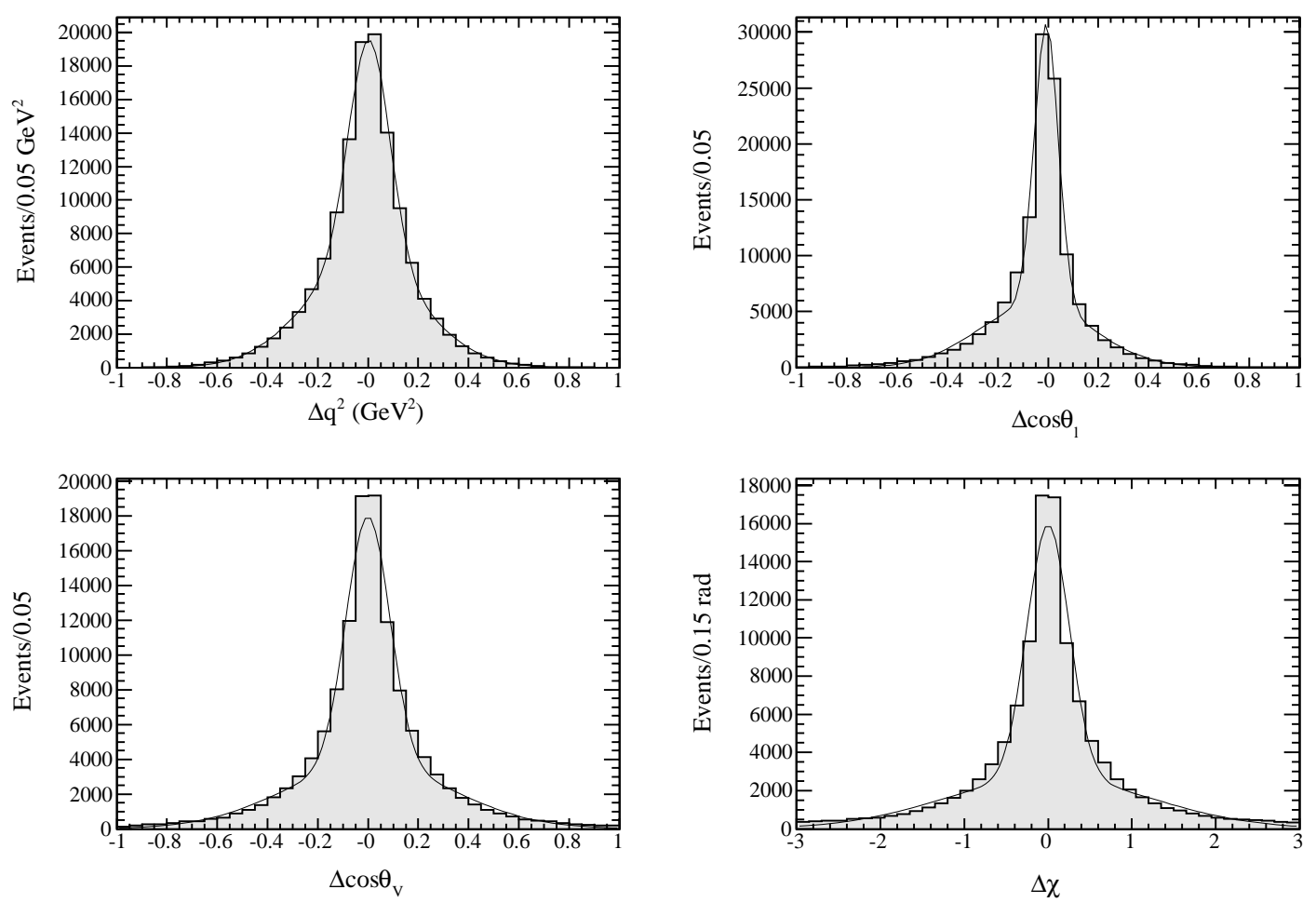

Figure 3.9: Distributions of the difference between the true and the reconstructed value of the four kinematic variables as obtained using simulated events. The fitted curve is the sum of two Gaussian distributions.

\begin{tabular}{|c|c|c|c|}
\hline variable & $\sigma_{1}$ & $\sigma_{2}$ & $\begin{array}{c}\text { fraction of the } \\
\text { narrower Gaussian }\end{array}$ \\
\hline$q^{2}$ & $0.082 \mathrm{GeV}^{2}$ & $0.233 \mathrm{GeV}^{2}$ & 0.44 \\
$\cos \left(\theta_{e}\right)$ & 0.048 & 0.227 & 0.47 \\
$\cos \left(\theta_{V}\right)$ & 0.087 & 0.334 & 0.49 \\
$\chi$ & $0.25(\mathrm{rad})$ & $1.22(\mathrm{rad})$ & 0.44 \\
\hline
\end{tabular}

Table 3.3: Standard deviation of the two Gaussian distributions used to fit resolutions on the kinematic variables. 
and the square of the $\pi^{+} \pi^{0}$ mass. This approach provides a measurement of the experimental resolution on $q^{2}$ and also on $\cos \theta_{l}$. The same procedure has been applied on simulated events and resolutions obtained on real and on simulated events have been compared. It has been found that, once the simulation is tuned such that the accuracy on the $D$ direction and the missing energy measurements are similar to data, then the reconstruction accuracy on $q^{2}$ is well reproduced by the corrected simulation. In the present analysis, this result has been used, and a dedicated $D_{s}^{+} \rightarrow \phi \pi^{+}$event sample has been analyzed to check the $D_{s}^{+}$direction and missing energy measurements in data and simulation (see Section4.1.2).

We compute the efficiency as function of each kinematic variable in the following way. We use a MC signal sample of $800 \mathrm{k}$ events. Using the reconstruction program, the simulated values of the variables are stored in two different datasets. The first one corresponds to the $\mathrm{MC}$ thruth level, thus it is free from any reconstruction effect. The second one contains the events that have been reconstructed, after having applied all the cuts of the analysis. Efficiency distributions are computed doing the ratio of the two histograms corresponding to the two datasets, for each variable, and are given in Figure 3.10. The signal reconstruction efficiency, including all cuts and the skim preselection, is of the order of $4 \%$.
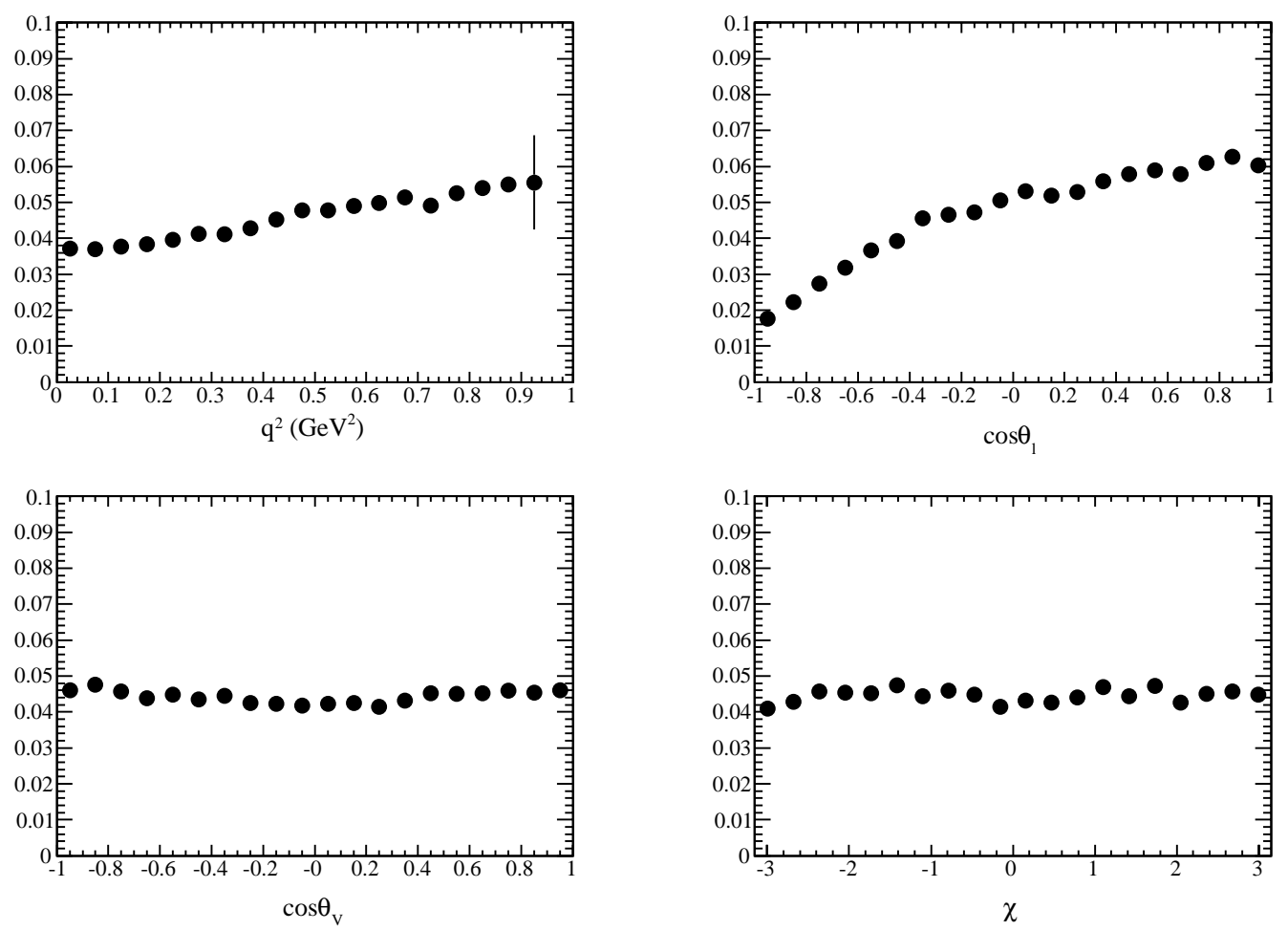

Figure 3.10: Efficiency distributions versus the generated values of the four kinematic variables. 


\section{Chapter 4}

\section{Adjustements to the simulation}

In a minute or two, the Caterpillar took the hookah out of its mouth and yawned once or twice, and shook itself. Then it got down off the mushroom, and crawled away into the grass, merely remarking as it went, 'One side will make you grow taller, and the other side will make you grow shorter.'

As explained in Section 3.1, in this analysis, the simulation plays an important role in the form factors measurement. In order to avoid biases, the data/MC agreement for the variables entering in the analysis, has to be checked for signal and background events, and corrections have to be defined if needed. This chapter presents different controls and adjustements applied to the simulation. The first part considers simulated signal events for which we use the $D_{s}^{+} \rightarrow \phi \pi^{+}$control sample. Then, events with additional leptons or large charge imbalance are studied with the same control sample. The last part details the background, with specific studies for each component.

\section{1 $D_{s}^{+} \rightarrow \phi \pi^{+}$events control sample}

There are differences between real and simulated $e^{+} e^{-} \rightarrow c \bar{c}$ events originating from the event generator or from a not-perfect simulation of the detector response. Events with a reconstructed $D_{s}^{+} \rightarrow \phi \pi^{+}$, named PHIPI in the following, have been used to measure and correct for some of these effects. Candidate events are selected using the same lists of particles as in the main analysis. $D_{s}^{+}$signals have been isolated and events in the sidebands 
of the $K^{+} K^{-}$mass distribution of the $\phi$ are used to evaluate effects from remaining background events under the peak (see Figure 4.1). To eliminate $D_{s}$ originating from $B$ meson decays, the $D_{s}$ momentum is required to exceed the maximum value in $B$ meson decays (corresponding to the process $\left.B \rightarrow D D_{s}\right)$ for which $E_{D_{s}}^{B}=\frac{1}{2}\left(m_{B}+\frac{m_{D_{s}}^{2}-m_{D}^{2}}{m_{B}}\right)=2.68 \mathrm{GeV}$. Such events correspond to $x_{D_{s}}=p_{D_{s}} / p_{D_{s}}^{\max } \leq 0.37 \cdot p_{D_{s}}^{\max }=\sqrt{E_{\text {beam }}^{2}-m_{D_{s}}^{2}}$ is the maximum $D_{s}$ momentum accessible in the center-of-mass system. $D_{s}$ mesons from $B$ decay can have a larger momentum if they are produced in $b \rightarrow u$ transitions but their fraction is small and we choose a cut at $x_{D_{s}}>0.44$ to avoid any contamination from $B$ decays. The $D_{s}^{+}$mass region is defined as $1.953 \mathrm{GeV} / \mathrm{c}^{2}$ to $1.983 \mathrm{GeV} / \mathrm{c}^{2}$. The background is subtracted using events from two sideband regions $\left(1.918 \mathrm{GeV} / \mathrm{c}^{2}\right.$ to $1.938 \mathrm{GeV} / \mathrm{c}^{2}$, and $1.998 \mathrm{GeV} / \mathrm{c}^{2}$ to $2.018 \mathrm{GeV} / \mathrm{c}^{2}$ ), as shown on Figure 4.1, rescaled to the number of background events in the signal region. Using only Run4, after background subtraction and keeping events with $\left|\cos \left(\theta_{\text {thrust }}\right)\right|<0.6$, there are 72793 candidates in data and 86739 in the simulation (without normalizing one sample to the other).

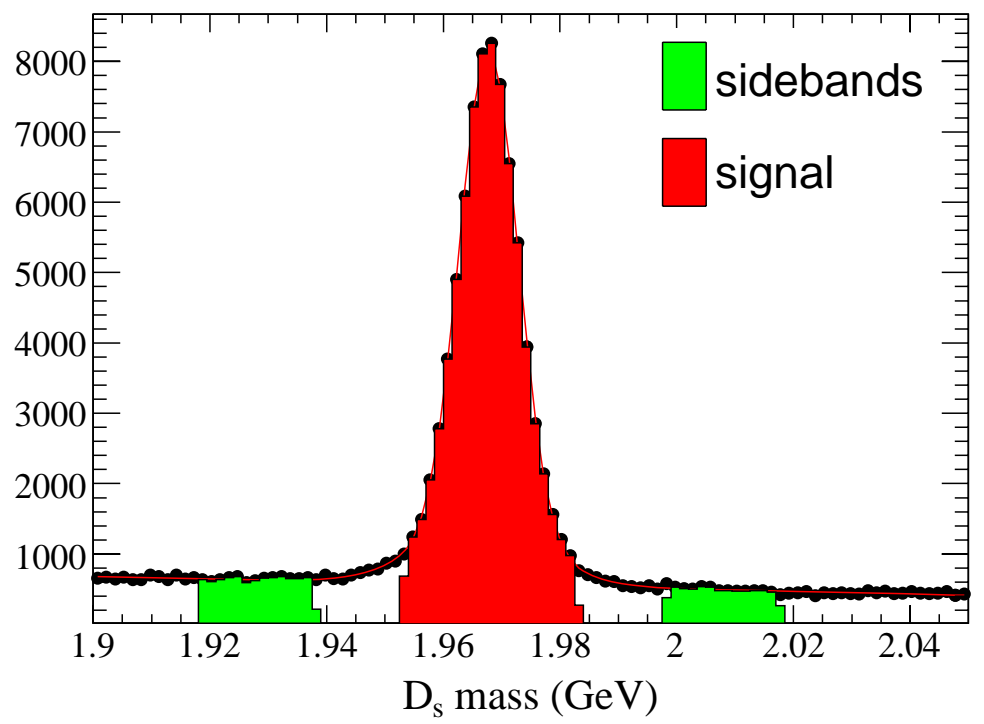

Figure 4.1: $D_{s}^{+} \rightarrow \phi \pi^{+}$mass distribution from data. The colored regions show the signal and sidebands ranges.

These events have been used to :

- compare and correct the distributions of the different variables entering in the Fisher discriminants;

- compare the reconstruction accuracy on the $D_{s}^{+}$direction and on the missing energy in data and MC. Measured differences have been corrected; 
- compare the missing energy measurement for events with additional lepton or large charge imbalance;

- compare the fraction of $D_{s}^{+}$events accompanied by a charged kaon or a $\phi$ meson. These events are contributing to the background and are analyzed in Section 4.3.4.

\subsubsection{Variables used in the Fisher discriminants}

Distributions of quantities entering into the definition of the Fisher discriminant variables, used to decrease the $B \bar{B}$ and $c \bar{c}$ background contributions are shown on Figure 4.2 and 4.3 for data and simulated events.
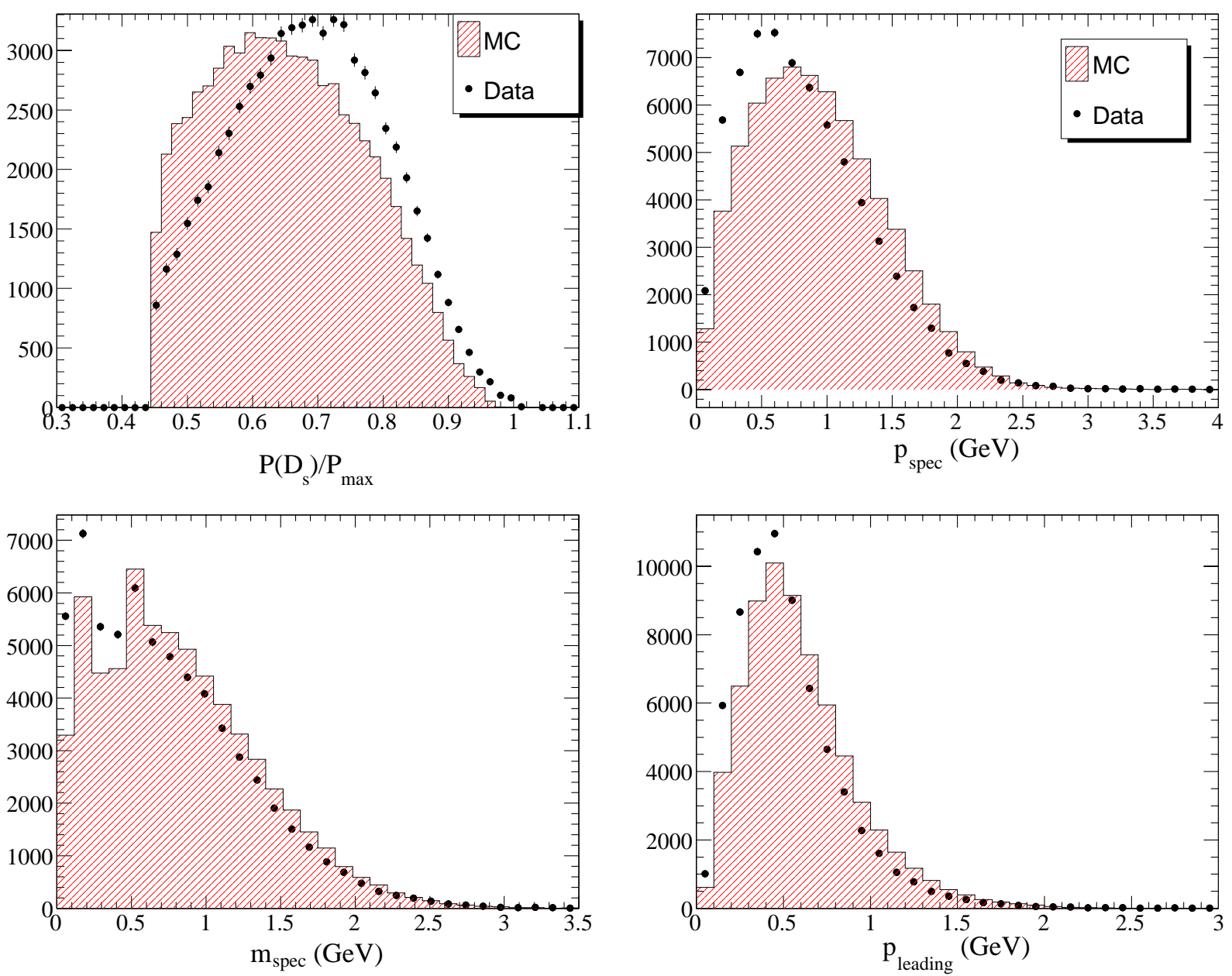

Figure 4.2: Comparison between data and simulation for the variables used in the Fisher discriminant analysis to decrease the cic event background level. 

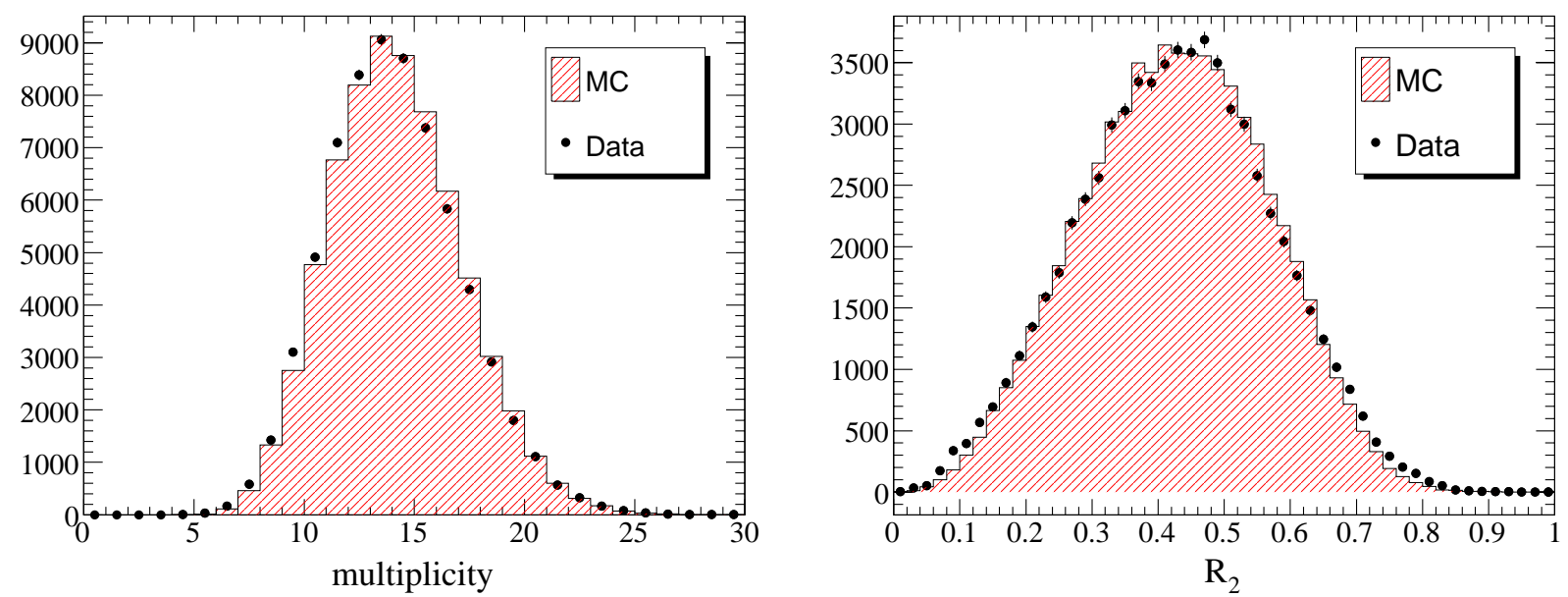

Figure 4.3: Distribution of the multiplicity and of the second Fox-Wolfram moment entering in the Fisher discriminant against $B \bar{B}$ events.

For $c \bar{c}$ events, these distributions depend on the c-quark fragmentation process and one can see that large differences exist between data and simulation. Consequently, the distribution of $x_{D s}$ measured with on-peak data has been used to define a first weight applied on simulated events in the following way. We compare the distribution of $x_{D s}$ in data, corrected for acceptance effects, with the distribution of $x_{D s}$ in simulated events at the Monte Carlo thruth level. The weight is computed by fitting a polynom to the ratio of the two normalized distributions.

After this first correction, the agreement between data and simulation for the other variables is checked. By an iterative process, we define successive weights, depending on the spectator system momentum, $R_{2}$, the multiplicity and the fragmentation, to reach a better agreement for all the variables. The resulting distributions, after all the iterations, are presented in Figures 4.4 and 4.5. 

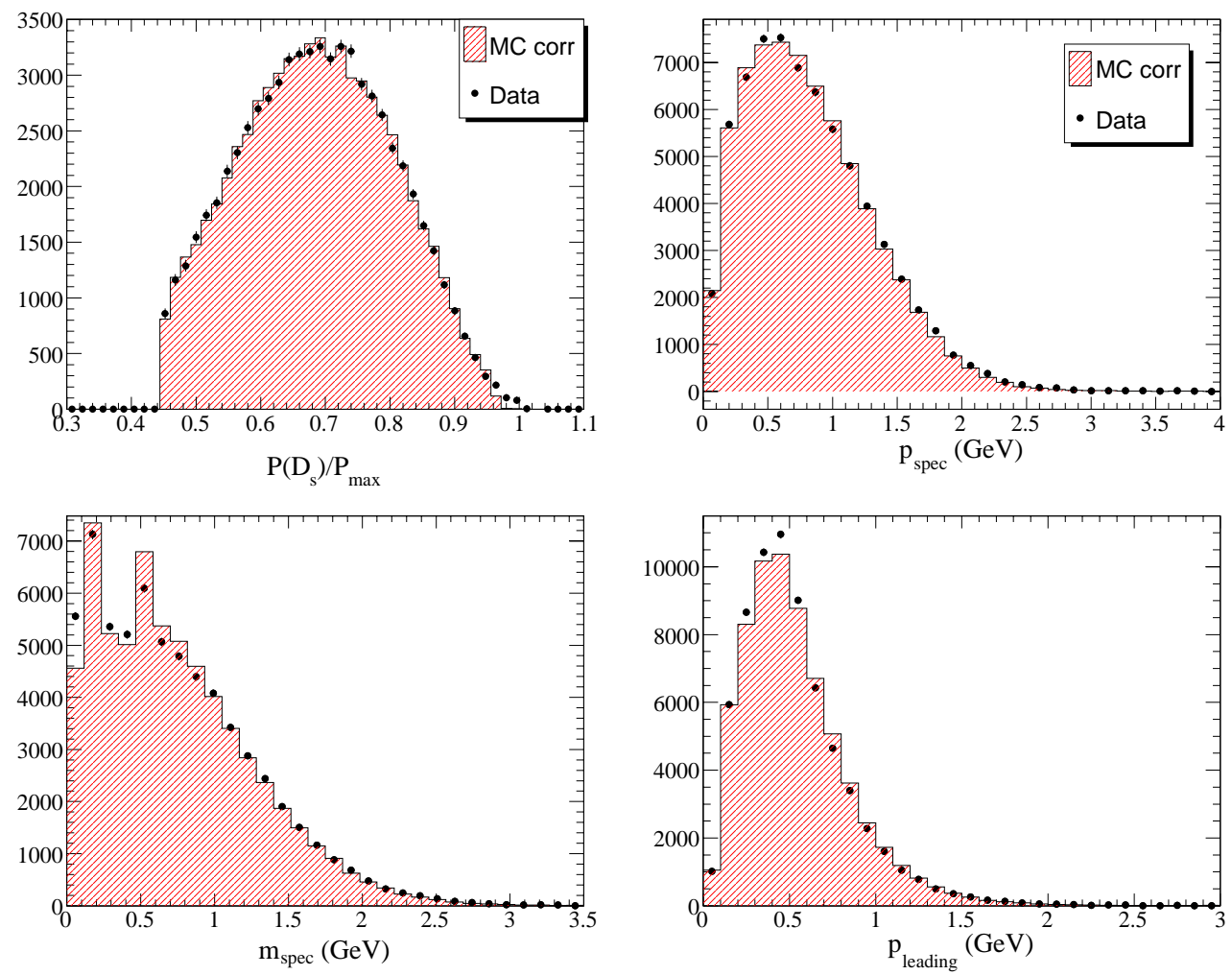

Figure 4.4: Comparison between real data and simulation for the variables used in the Fisher discriminant analysis after all corrections.
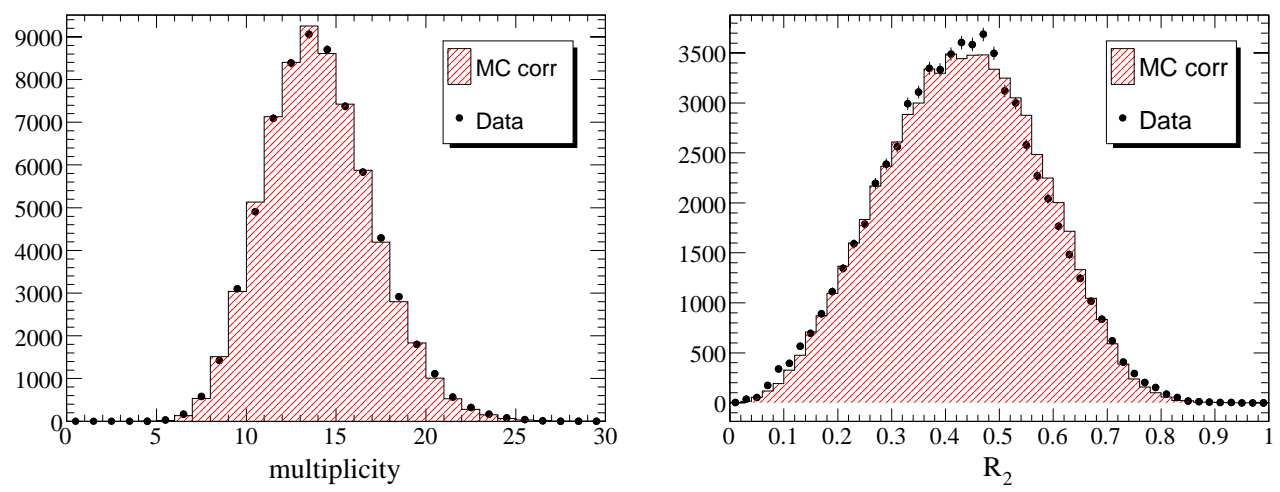

Figure 4.5: Distribution of the second Fox-Wolfram moment and of the multiplicity after having applied corrections on simulated events. 
As shown on Figure 4.6 using simulated events, the momentum distribution of the $D_{s}^{+}$ in PHIPI events is quite different from the one in $D_{s}^{+} \rightarrow \phi e^{+} \nu_{e}$ events. The difference between these two spectra comes mainly from the difference of efficiencies for the two channels, which are displayed on Figure 4.7. The momentum dependence of the efficiency for the $D_{s}^{+} \rightarrow \phi e^{+} \nu_{e}$ channel is explained by the use of cuts, like the one on the Fisher discriminant against $c \bar{c}$ events, that select $D_{s}$ candidates with high momentum. In order to verify if the corrections defined with PHIPI events, on the variables that enter the Fisher discriminant, are appropriate for the semileptonic channel, the following test is done. It consists in comparing the $D_{s}^{+}$momentum distributions measured for $\phi \pi$ and $\phi e \nu$ events, before and after applying corrections on the variables entering the Fisher discriminants. From the simulated distributions (without applying any corrections), we define a weight $w_{p\left(D_{s}\right)}$ depending on the $D_{s}^{+}$momentum so that the $D_{s}^{+}$momentum distribution in PHIPI events is similar to the one obtained for the semileptonic channel. The weighted momentum distribution of PHIPI events is shown on Figure 4.6 and is compared with the momentum distribution of $D_{s}^{+} \rightarrow \phi e^{+} \nu_{e}$ events. The PHIPI distribution weighted with $w_{p\left(D_{s}\right)}$ reproduces well the distribution of semileptonic events. On the other hand, starting from the original distributions, we apply the corrections depending on the Fisher variables, $w_{F i s h}$, on PHIPI and semileptonic events. The corrected distributions are shown in Figure 4.8. We then apply the weight $w_{p\left(D_{s}\right)}$ on the PHIPI events corrected with $w_{F i s h}$. On the same figure, we observe that the resulting distribution of PHIPI events reproduces well the distribution of semileptonic events corrected with $w_{\text {Fish. }}$. This test demonstrates that starting from the $D_{s}^{+}$momentum distribution of $D_{s}^{+} \rightarrow \phi e^{+} \nu_{e}$ decays would have lead to the same corrections as the ones defined with $D_{s}^{+} \rightarrow \phi \pi$ events.

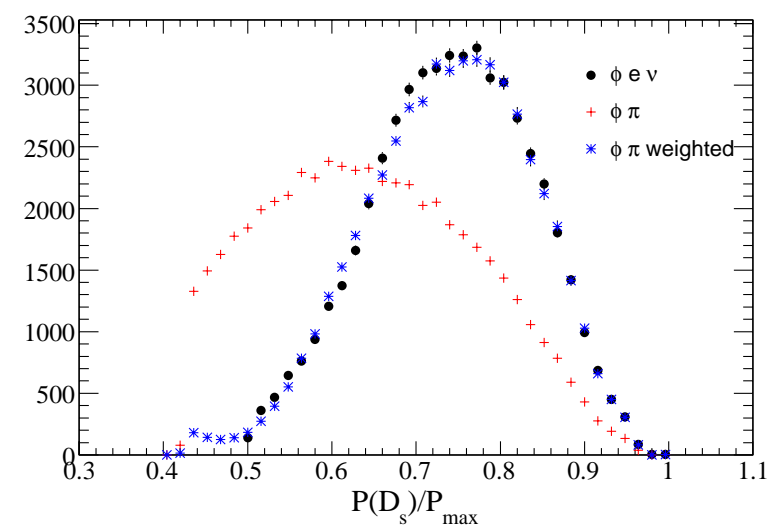

Figure 4.6: Comparison between $D_{s}^{+}$momentum distribution in $D_{s}^{+} \rightarrow \phi e^{+} \nu_{e}$ (black dots) and $D_{s}^{+} \rightarrow \phi \pi^{+}$(red crosses) selected events. The distribution of PHIPI events weighted with $w_{p\left(D_{s}\right)}$ is also shown (blue stars). These distributions are obtained from simulation. 

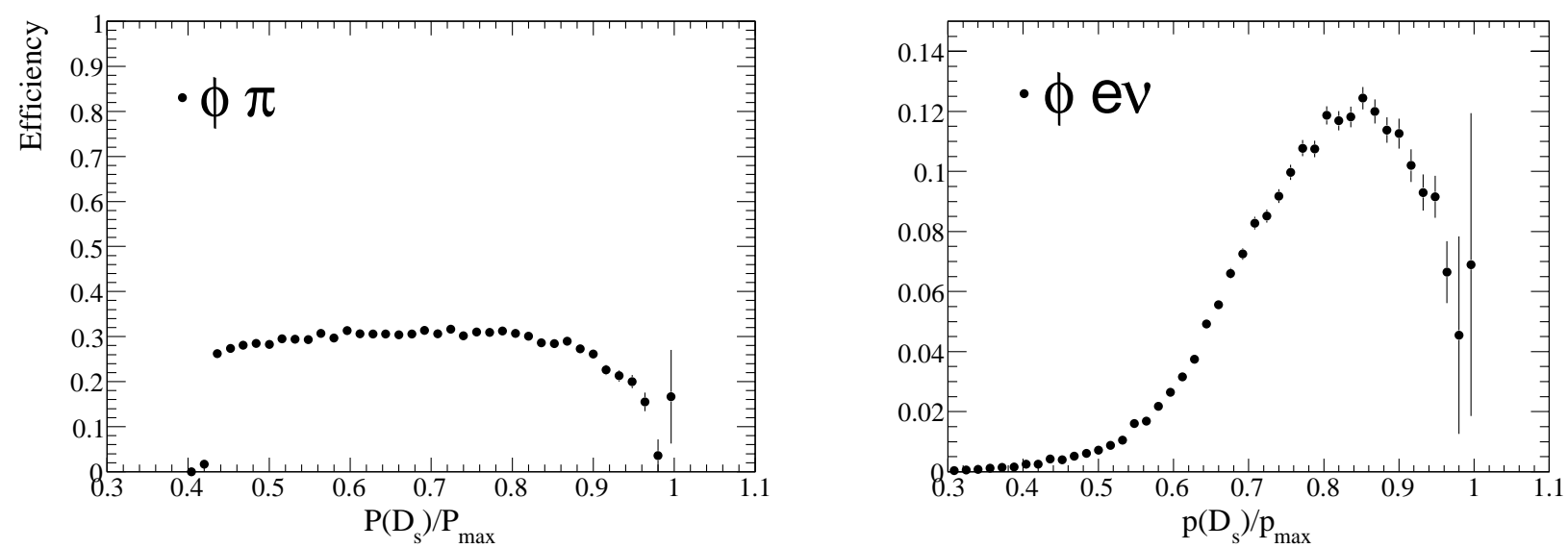

Figure 4.7: Efficiency distributions versus $x_{D_{s}^{+}}$for the $D_{s}^{+} \rightarrow \phi \pi^{+}$channel (left) and the $D_{s}^{+} \rightarrow \phi e^{+} \nu_{e}$ channel (right).

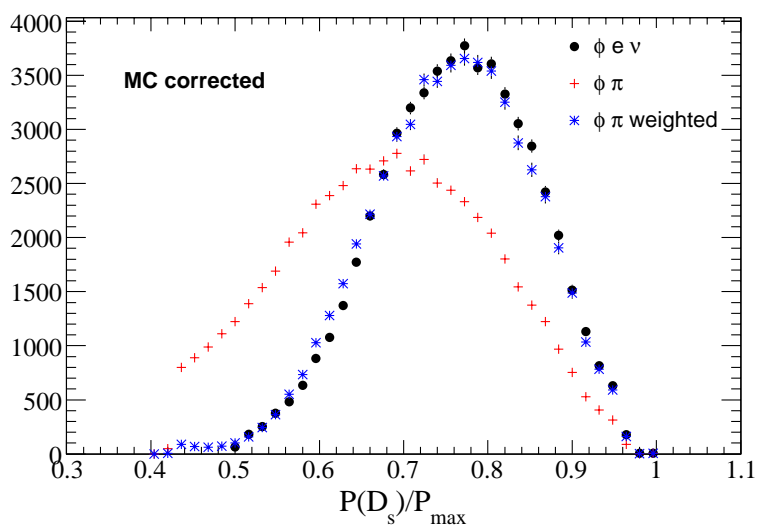

Figure 4.8: Comparison between $D_{s}^{+}$momentum distribution in $D_{s}^{+} \rightarrow \phi e^{+} \nu_{e}$ (black dots) and $D_{s}^{+} \rightarrow \phi \pi^{+}$(red crosses) selected events. These distributions are obtained from simulation and corrections depending on the Fisher variables ( $w_{\text {Fish. }}$ ) are applied. The distribution of PHIPI events weighted by $w_{F i s h} \times w_{p\left(D_{s}\right)}$ is also shown (blue stars) and reproduces well the corrected distribution of $D_{s}^{+} \rightarrow \phi \pi$ events. 


\subsection{2 $\quad D_{s}^{+}$direction and missing energy}

As explained in section 3.3, the quantities used to compute the $D_{s}^{+}$momentum in the reconstruction of $D_{s}^{+} \rightarrow \phi e^{+} \nu_{e}$ events are the missing energy in the lepton hemisphere and the $D_{s}^{+}$ direction. The latter is estimated by summing the momentum of all particles reconstructed in the event, except those coming from the $D_{s}^{+}$decay. The resulting distributions of these quantities in data and in the simulation are compared and verified using PHIPI events. We can then:

- correct the missing energy in the candidate hemisphere. In PHIPI events, as there is no missing particle from the $D_{s}^{+}$decay, the missing energy should be centered at zero if no particle is lost or badly measured. The neutrino energy is estimated by subtracting, from the missing energy measured in the hemisphere, the corresponding average value obtained with PHIPI events. The width of the distribution is used to estimate the resolution on this quantity;

- correct the estimated $D_{s}^{+}$direction, with the one measured using the $D_{s}^{+}$decay products, and assess the corresponding error.

These corrections are parameterized as a function of the missing energy measured in the opposite hemisphere $\left(\right.$ Emiss $\left._{\text {opp }}\right)$. The variable Emiss $_{\text {opp }}$ is used as an indicator of the quality of the energy reconstruction in the event. It is observed, as expected, that the resolution on the $D_{s}^{+}$direction and missing neutrino energy decreases as Emiss $s_{\text {opp }}$ increases (see Figure 4.12). Distributions in data and MC events are compared and measured differences are corrected. These comparisons correspond to averages over the direction and the energy of the $D_{s}$ meson. For the two samples, the same cut on $\cos \theta_{\text {thrust }}$ has been applied and a weight on PHIPI events is also used so that the $D_{s}^{+}$momentum distribution in this exclusive channel is similar to the one obtained for the semileptonic channel.

Distributions of the missing energy in the same and in the opposite hemisphere, for the data and the simulation can be seen on Figure 4.9, with the corresponding ratios data over MC. Simulation has been normalized to the data luminosity. These distributions take into account the corrections defined in section 4.1.1. The agreement is rather good between data and the simulation with a slightly better resolution for the latter. To correct this effect, we define a smearing, applied on simulated events, which depends on the missing energy measured in the opposite hemisphere. We call smearing, the procedure which consists in the transformation of a distribution by adding a random number, generated according to a Gaussian distribution, to the considered variable. The smearing applied on the missing energy is defined by the parameters given in Table 4.1. The corrected distributions can be 
seen on Figure 4.10. In the following, this smearing, of the order of $130 \mathrm{MeV}$ is applied to the Monte Carlo signal.
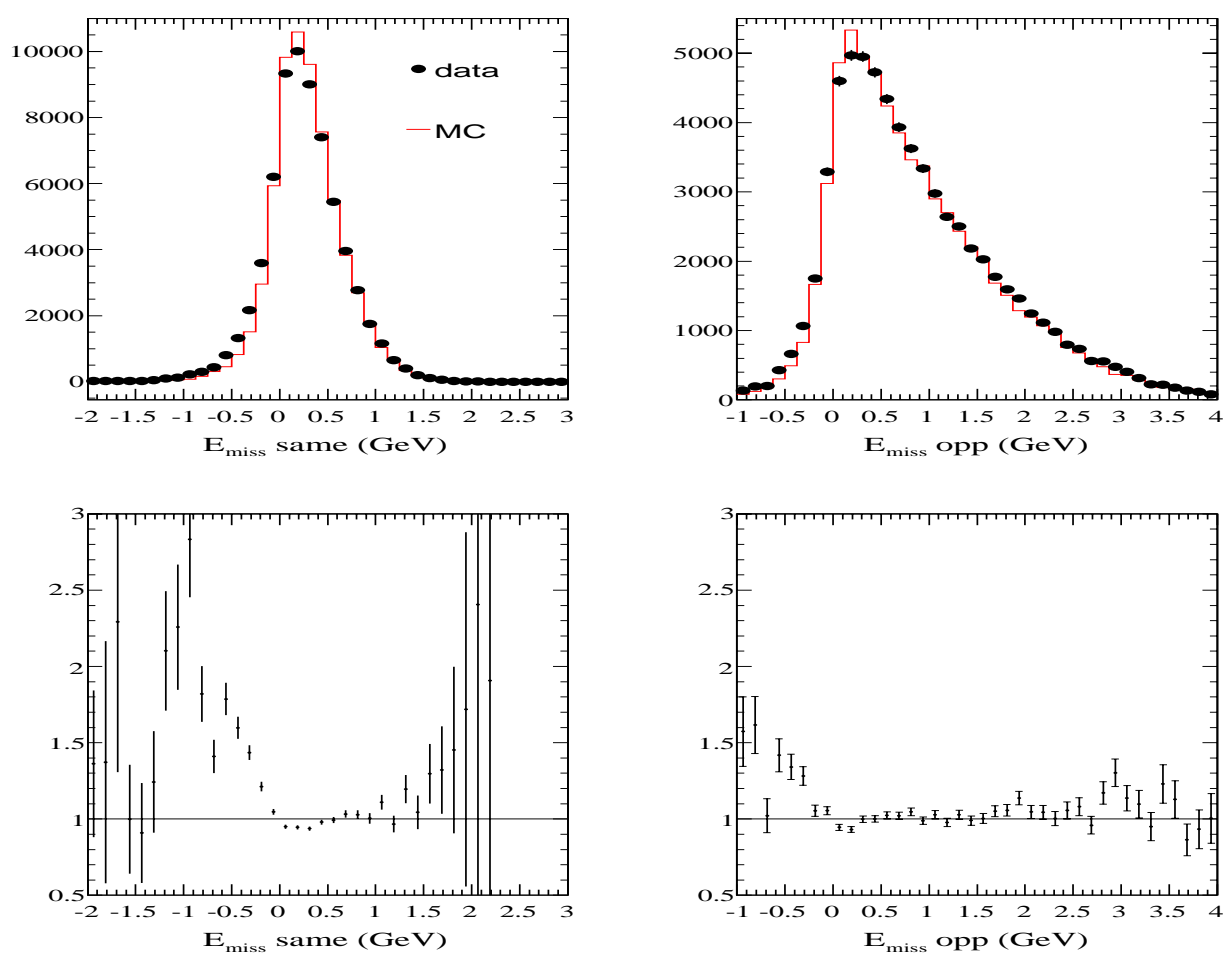

Figure 4.9: Comparison between real and simulated events corrected for differences in fragmentation. The upper plots are the missing energy in the same hemisphere (left) and in the opposite hemisphere (right) that contains the candidate. The lower plots are the corresponding ratio data over $M C$.

The $D_{s}^{+}$direction distributions are shown on Figure 4.11. The widths of the distributions corresponding to the difference between the values of the angles $\theta$ and $\phi$ obtained from the reconstructed $D_{s}^{+}$decay and those estimated from all the other particles in the event, depend on the missing energy registered in the opposite hemisphere. 


\begin{tabular}{|c|c|c|}
\hline Range $(\mathrm{GeV})$ & mean $(\mathrm{GeV})$ & $\sigma(\mathrm{GeV})$ \\
\hline$E_{\text {miss }}$ opp. $\leq 1.5$ & -0.03 & 0.130 \\
$1.5<E_{\text {miss }}$ opp. $\leq 1.75$ & -0.01 & 0.129 \\
$1.75<E_{\text {miss }}$ opp. $\leq 2$ & -0.02 & 0.131 \\
$2<E_{\text {miss }}$ opp. $\leq 2.25$ & 0.0 & 0.189 \\
$2.25<E_{\text {miss }}$ opp. $\leq 2.5$ & 0.0 & 0.130 \\
$2.5<E_{\text {miss }}$ opp.$\leq 2.75$ & +0.02 & 0.156 \\
\hline
\end{tabular}

Table 4.1: Definition of the smearing added on the missing energy in the lepton hemisphere. For each interval of the missing energy in the opposite hemisphere, we add a smearing following a Gaussian distribution with a defined mean and $\sigma$.
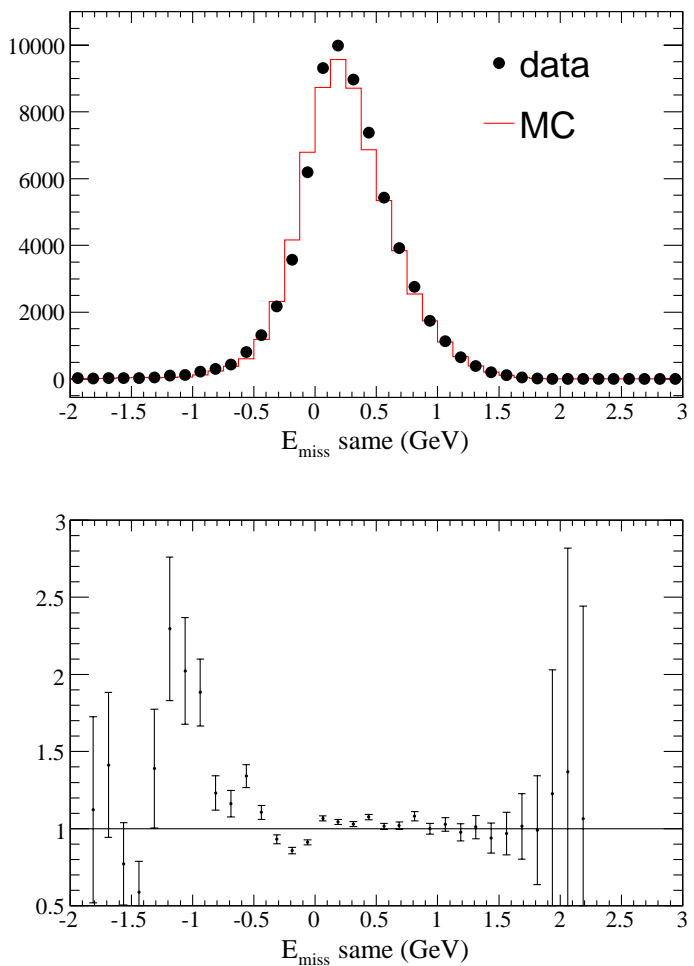

Figure 4.10: Comparison of the missing energy in the same hemisphere between real and simulated events corrected for fragmentation after a smearing correction is applied on the missing energy in the candidate hemisphere. 

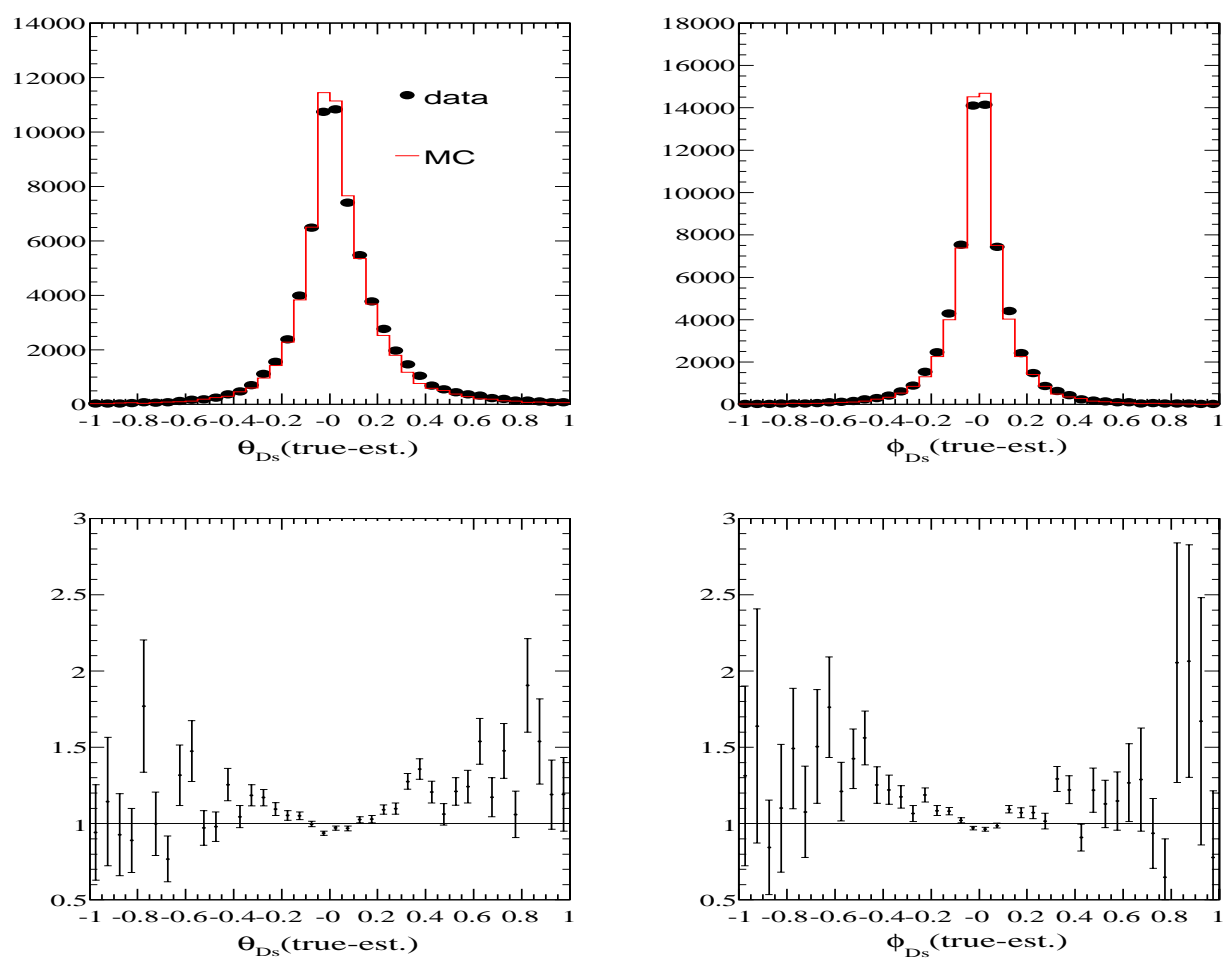

Figure 4.11: Difference between the $D_{s}^{+}$angles obtained from the decay products and from the other particles measured in each events. Corrections have been applied on $M C$ events but no additionnal smearing. 
Parameterizations of measured bias and accuracy have been obtained from real data, versus Emiss $_{\text {opp }}$ by fitting Gaussian distributions for events selected in 20 Emiss $_{\text {opp }}$ bins. The fit results can be seen on Figure 4.12.

The parameterization obtained for the $D_{s}^{+}$direction (radians) is:

$$
\begin{aligned}
\theta_{D_{s}^{+}} & =\theta_{D_{s}^{+}}^{\text {est. }}-6.39 .10^{-4}+0.04506 * \mid \text { Emiss }_{\text {opp }}|-0.00536 *| \text { Emiss }\left._{\text {opp }}\right|^{2} \\
\sigma_{\theta_{D_{s}^{+}}} & =0.05269+0.13983 * \mid \text { Emiss }_{\text {opp }}|-0.01197 *| \text { Emiss }\left._{\text {opp }}\right|^{2} \\
\phi_{D_{s}^{+}} & =\phi_{D_{s}^{+}}^{\text {est. }}
\end{aligned}
$$

For the missing energy in the candidate hemisphere $\left(\mathrm{GeV} / \mathrm{c}^{2}\right)$ :

Emiss $_{\text {opp }} \leq 2.5 \mathrm{GeV} / \mathrm{c}^{2}:$

$$
\begin{aligned}
E_{\nu}= & E_{m i s s}^{\text {same }}-\left(0.004489+0.570101 * \text { Emiss }_{\text {opp }}+0.012229 * \text { Emiss }_{o p p}^{2}\right. \\
& -0.422964 \text { Emiss }_{\text {opp }}^{3}+0.160211 * \text { Emiss }_{\text {opp }}^{4}+0.106162 * \text { Emiss }_{\text {opp }}^{5} \\
& \left.-0.076132 * \text { Emiss }_{\text {opp }}^{6}+0.012412 * \text { Emiss }_{\text {opp }}^{7}\right)
\end{aligned}
$$

Emiss $_{\text {opp }}>2.5 \mathrm{GeV} / \mathrm{c}^{2}: \mathrm{E}_{\nu}=\mathrm{E}_{\text {miss }}^{\text {same }}-0.5$

And,

$$
\sigma_{E_{\nu}}=0.238792+1.25876 e-01 * \mid E_{\text {Emiss }} \text { opp }\left.|-2.58855 e-02 *| E_{\text {Emiss }}{ }_{\text {opp }}\right|^{2}
$$

In these expressions the superscript "est." refers to the estimated measurement explained in Section 3.3 and $\sigma_{x}$ gives the expected uncertainty for this measurement. Input values in the mass constrained fit include these corrections.

It has been verified with $D^{*+} \rightarrow D^{0} \pi^{+}, D^{0} \rightarrow K^{-} \pi^{+} \pi^{0}$ events, that the $q^{2}$ resolution in data was well reproduced by the simulation after having applied this type of corrections ([46]). 

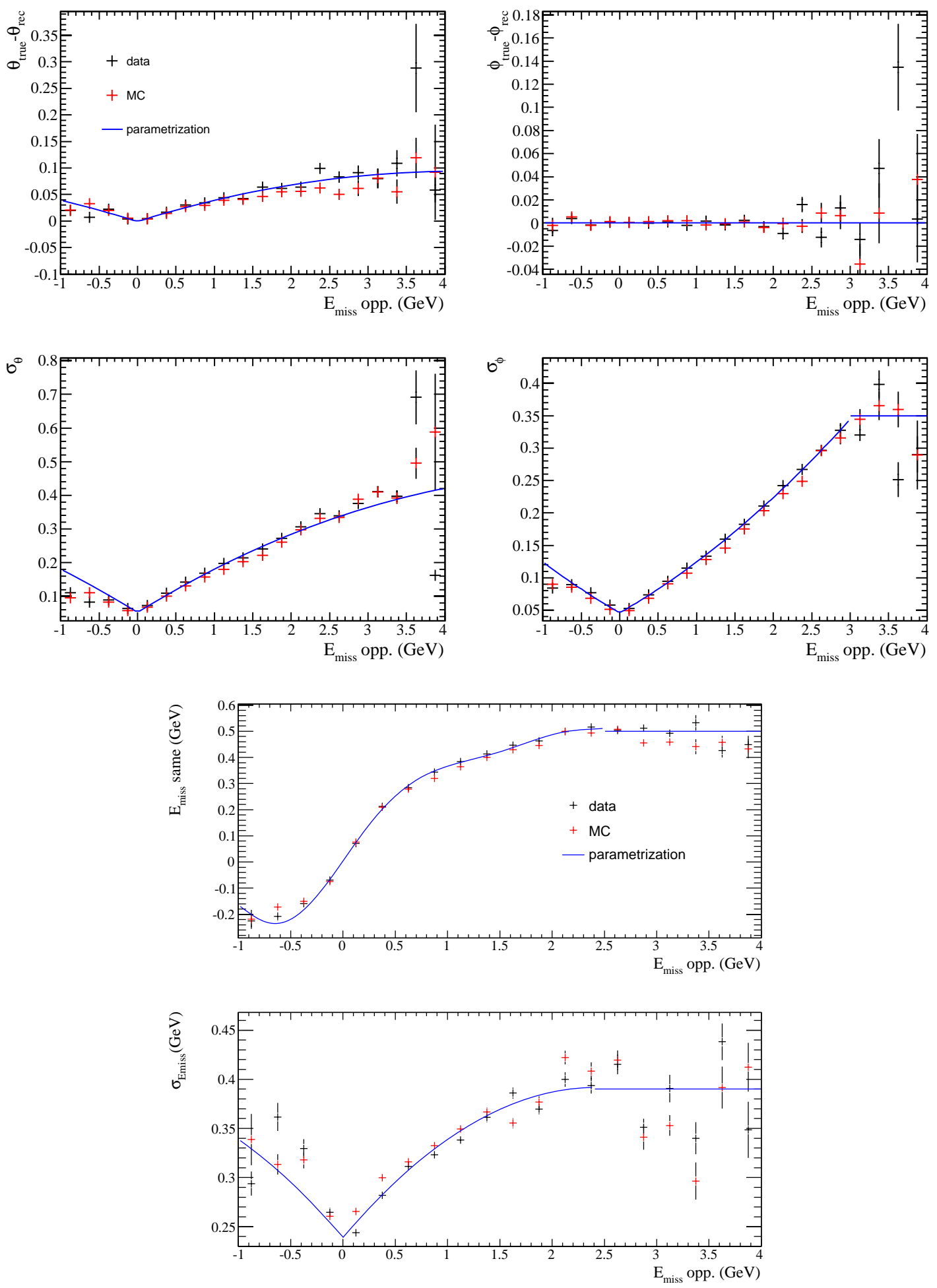

Figure 4.12: Central value and $\sigma$ of the Gaussian distribution fitted as a function of the missing energy in the opposite hemisphere. Data is used to define the parameterization. 


\subsection{Study of events with additional electrons or large charge imbalance}

PHIPI events are also used to study the energy reconstruction of several types of events which can have a potential effect on the missing energy measurement. In this study, on-peak and off-peak events are used and $78000 D_{s}$ signal events are selected in data. Distributions of the missing energy measurement, in the same and in the opposite hemisphere, are compared in data and in the simulation. Two events classes have been considered. Events with an additional electron candidate are enriched in charm semileptonic decays in the opposite event hemisphere and one expects a larger missing energy in that hemisphere. Events with large charge imbalance must correspond to tracks that have been missed or contain background additional tracks. In this case also it is expected that the missing energy measurement is degraded.

Simulated events are weighted to correct for the differences between data and simulation measured for the $D_{s}$ momentum, $R_{2}$, the total multiplicity and production characteristics of the spectator system.

To estimate the neutrino energy and to be in the same conditions as in $D_{s} \rightarrow \phi e^{+} \nu_{e}$ analysis, we apply also a correction which depends on the energy measured in the hemisphere opposite to the candidate. All distributions are obtained after subtracting the background contribution using the $D_{s}$ sidebands.

\subsubsection{Events with an additional electron}

The number of additional electrons is given in Figure 4.13. In data, the fraction of events with at least one additional electron is $(9.9 \pm 0.1) \%$ and a similar value is measured in the simulation $(10.2 \pm 0.1) \%$

Missing energy distributions have been compared in Figure 4.14 for events with or without an electron and considering separately data and simulation.

\begin{tabular}{|c|c|c|c|c|c|}
\hline Data & $\begin{array}{c}\bar{E}_{\text {miss. }} \\
\mathrm{GeV}\end{array}$ & $\begin{array}{c}\sigma_{E} \\
\mathrm{GeV}\end{array}$ & $\mathrm{MC}$ & $\begin{array}{c}\bar{E}_{\text {miss. }} \\
\mathrm{GeV}\end{array}$ & $\begin{array}{c}\sigma_{E} \\
\mathrm{GeV}\end{array}$ \\
\hline no el. & 0.116 & 0.446 & & 0.117 & 0.410 \\
$\geq 1$ el. & 0.089 & 0.447 & & 0.087 & 0.419 \\
\hline
\end{tabular}

Table 4.2: Average value and standard deviation of the missing energy reconstructed in the $D_{s}$ hemisphere. Events with or without an electron have been separated.

The missing energy in the candidate hemisphere has similar distributions for events with 


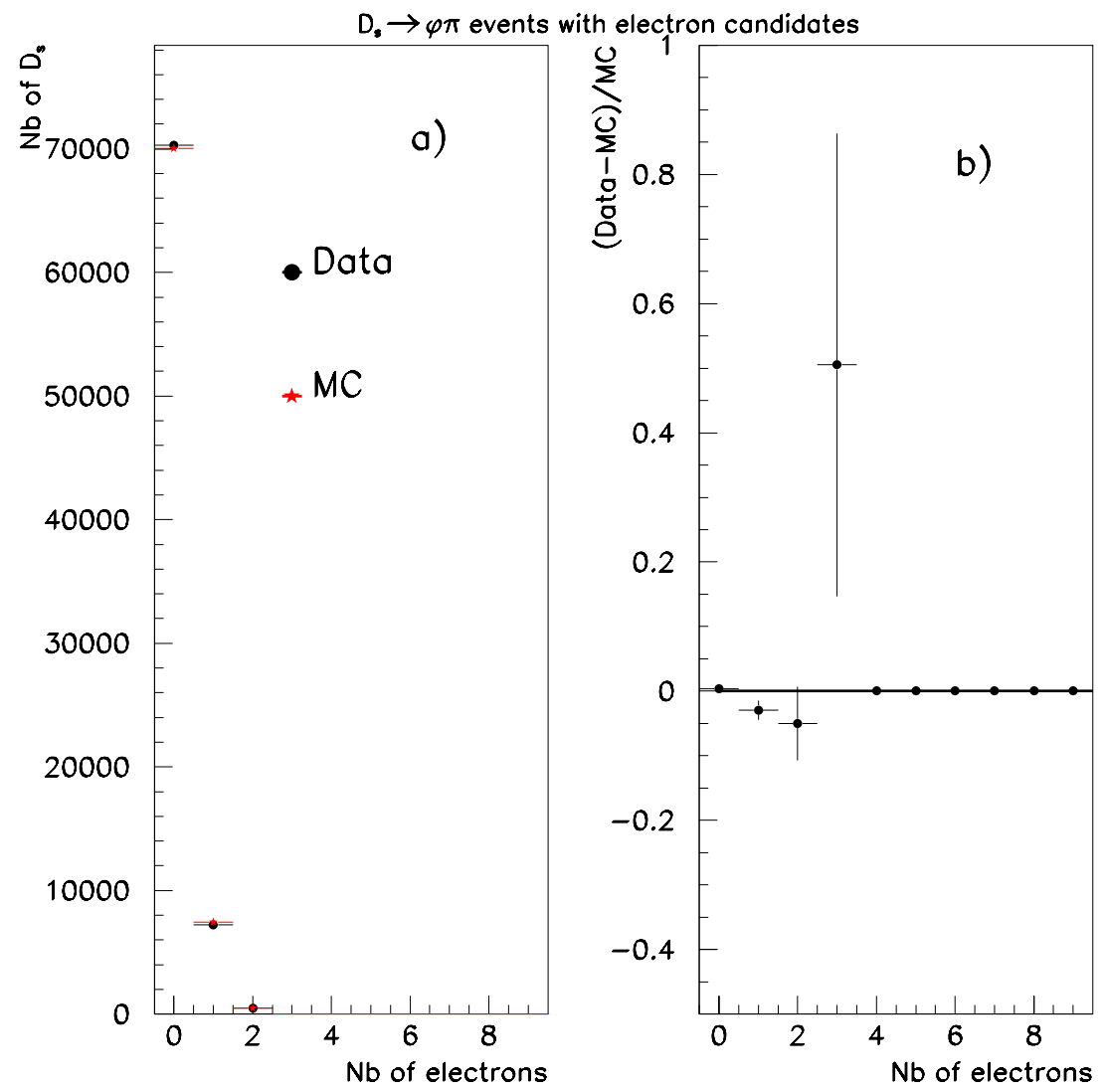

Figure 4.13: Number of electron candidates in events tagged with a $D_{s}$. 

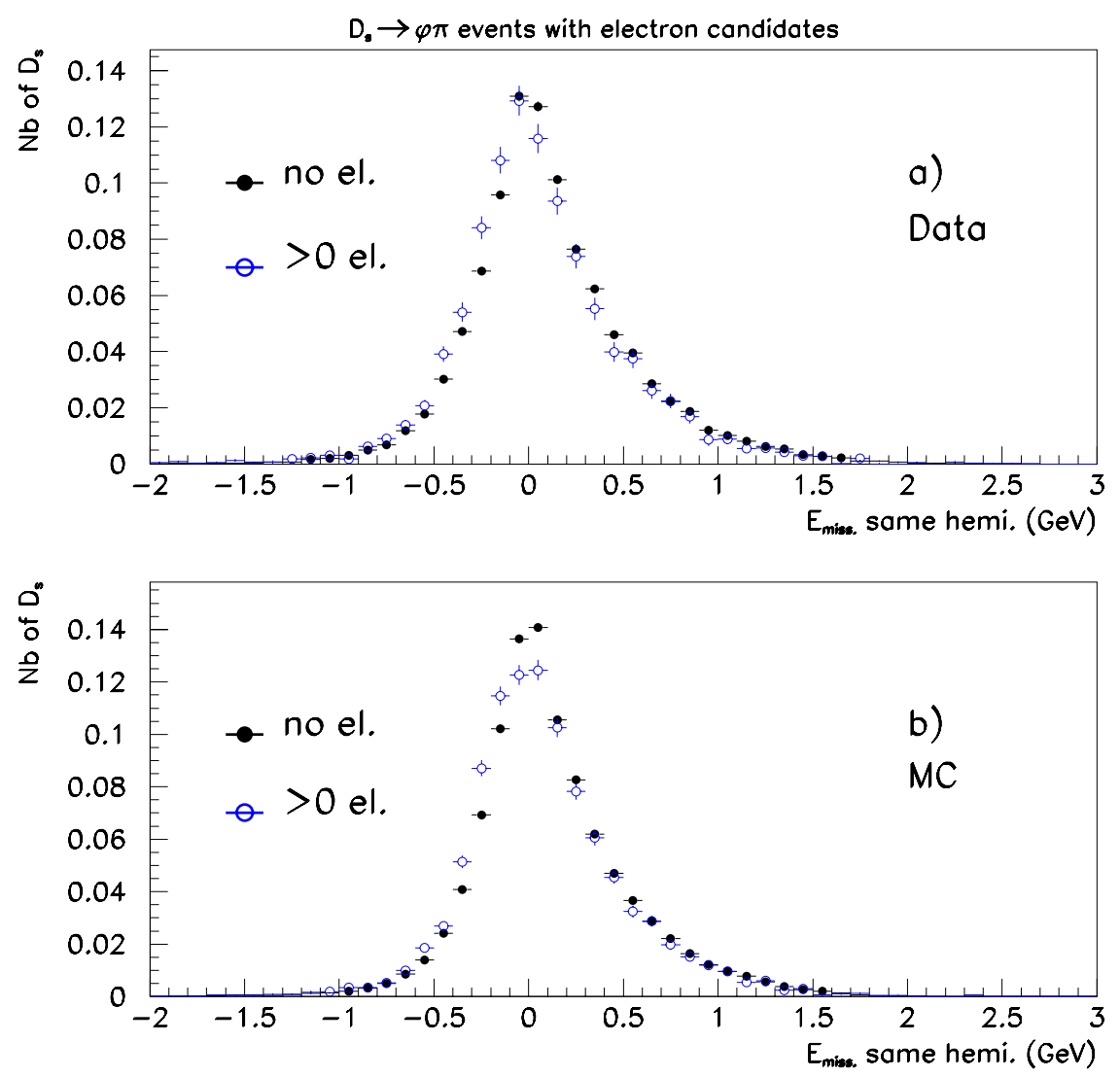

Figure 4.14: Reconstruction of the missing energy in the $D_{s}$ hemisphere in data (a) and in the simulation (b). Events with at least one electron candidate have been separated. Distributions are normalized to unity. 
or without an additional electron. The behaviour of the simulation is also rather similar to data, as shown in Table 4.2. The missing energy measurement is a bit better in the simulation this is why in the analysis we have added a smearing on this quantity.

In Figure 4.15 we have also compared the missing energy measured in the opposite hemisphere for events with or without an electron, separately for data and simulation. There is a clear signal of missing energy in events with an electron and this is well reproduced by the simulation. From Figure 4.14 above, one has verified that this effect has no influence on the energy determination in the signal hemisphere.
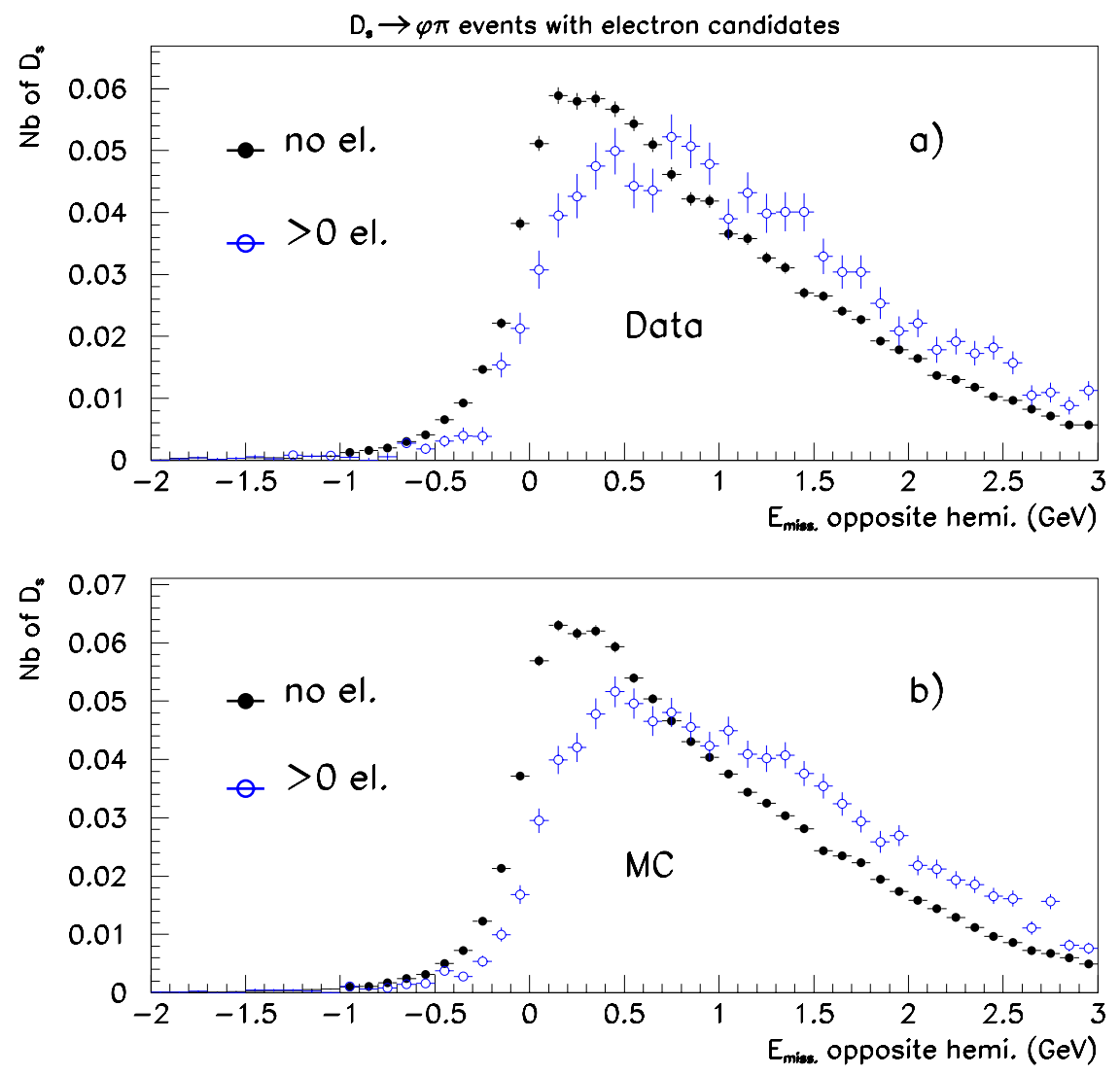

Figure 4.15: Reconstruction of the missing energy in the hemisphere opposite to the $D_{s}$ in data (a) and in the simulation (b). Events with at least one electron candidate have been separated. Distributions are normalized to unity. 


\subsubsection{Events with bad charge imbalance}

The distribution of the event charge $\left(\Delta Q=n^{+}-n^{-}\right)$is given in Figure 4.16. The fraction of events with $\Delta Q \geq 2$ is $(10.0 \pm 0.1) \%$ in data and $(9.3 \pm 0.1) \%$ in the simulation. As in the previous study we have compared the missing energy measurement in the $D_{s}$ hemisphere and in the opposite, for events with $\Delta Q \leq 1$ and $\Delta Q \geq 2$, separately for data and simulation.

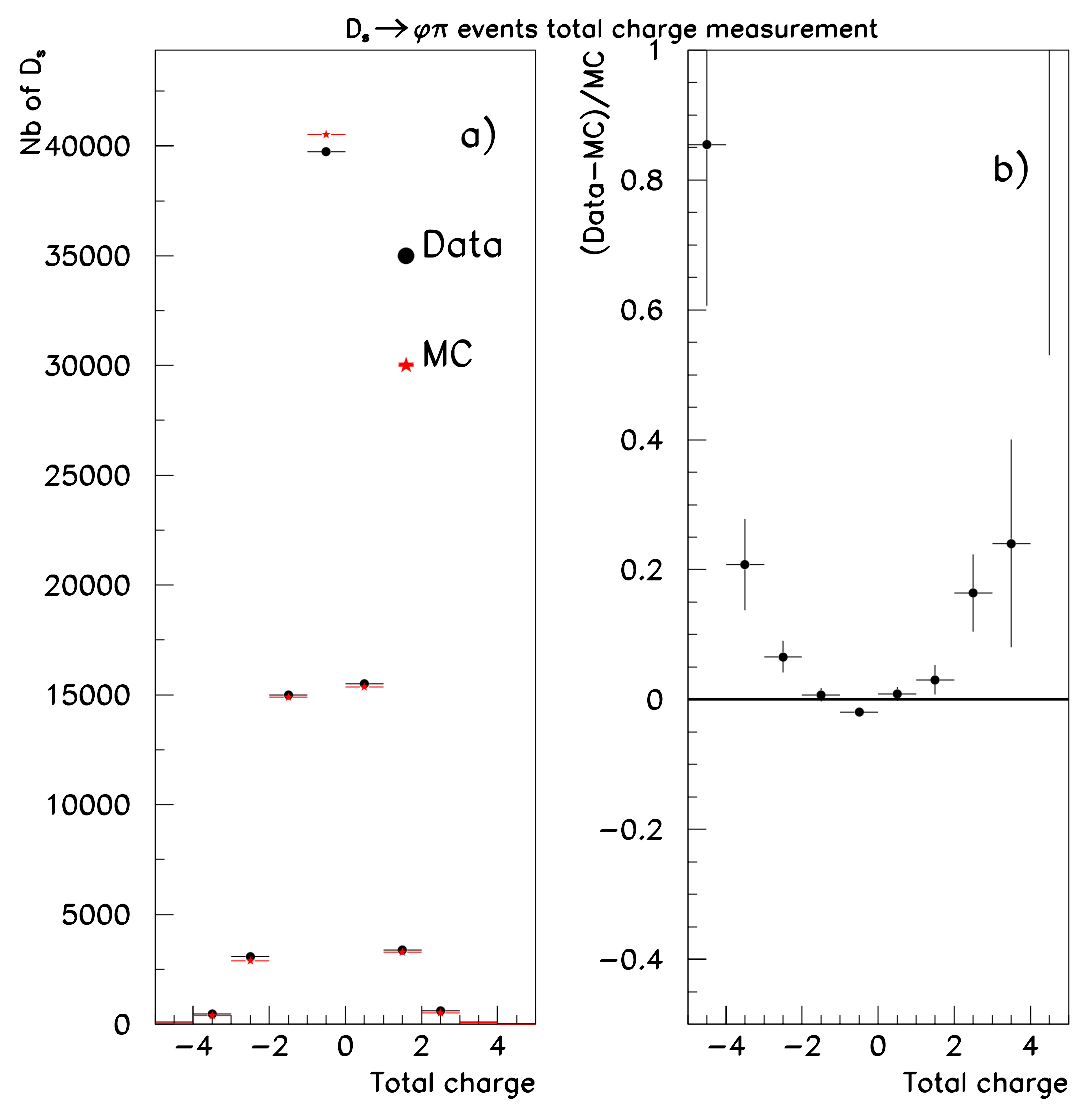

Figure 4.16: Charge imbalance distribution in events tagged with a $D_{s}$.

As compared with the previous study we note that the missing energy measurement in the same hemisphere deteriorates for events with $\Delta Q \geq 2$ (see Table 4.3 and Figure 4.17).

Figure 4.18 gives the distributions of the missing energy measured in the opposite hemisphere. Energy is better conserved for events with $\Delta Q \leq 1$. The simulation reproduces correctly these effects.

To improve the energy reconstruction in the $D_{s}^{+} \rightarrow \phi e^{+} \nu_{e}$ analysis, events with $\Delta Q \geq 2$ can be eliminated. At present, as the simulation agrees with data on the fraction and on 

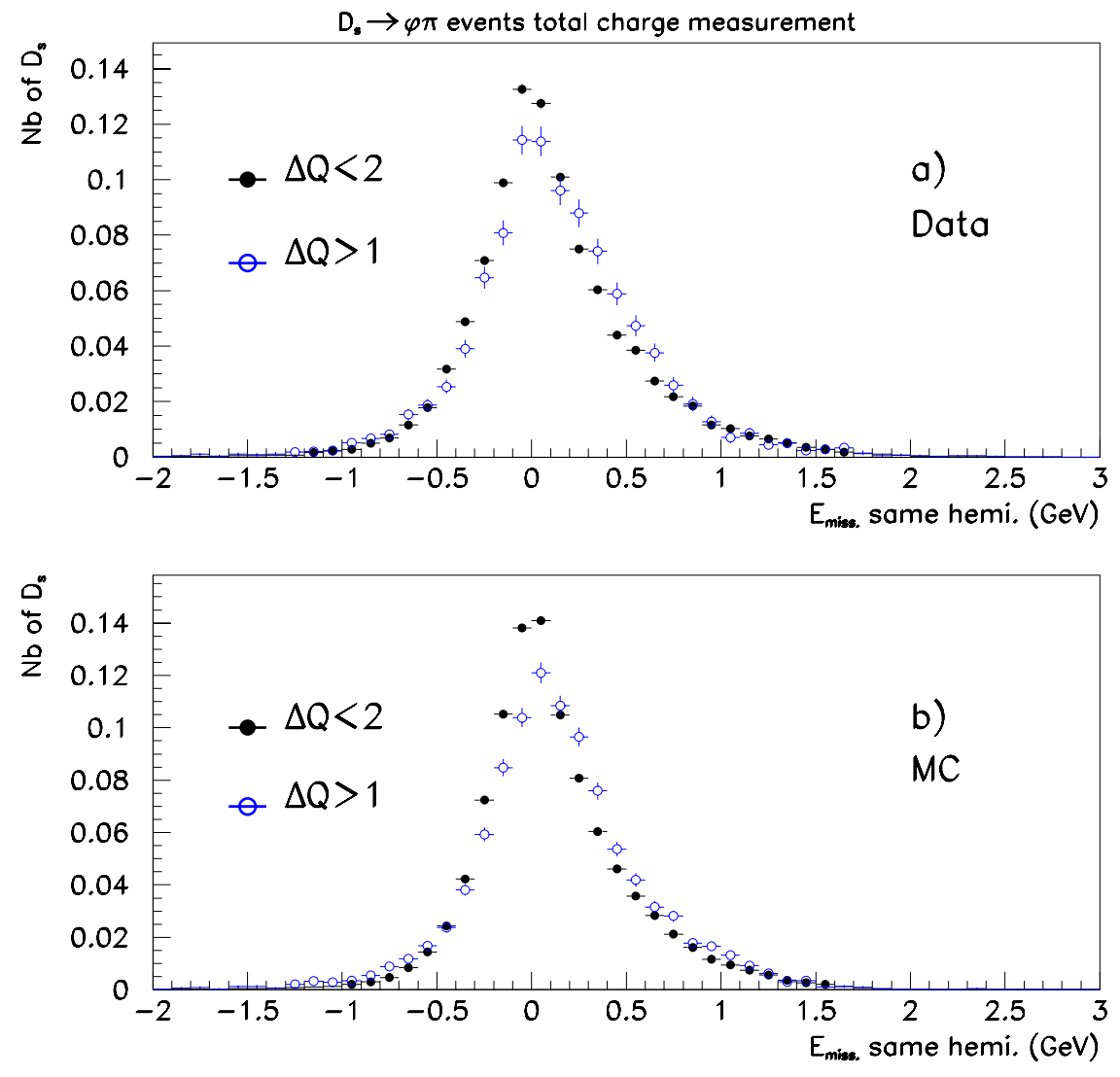

Figure 4.17: Reconstruction of the missing energy in the $D_{s}$ hemisphere in data (a) and in the simulation (b). Events with $\Delta Q \geq 2$ have been separated. Distributions are normalized to unity.

\begin{tabular}{|c|c|c|c|c|c|}
\hline Data & $\begin{array}{c}\bar{E}_{\text {miss. }} \\
\mathrm{GeV}\end{array}$ & $\begin{array}{c}\sigma_{E} \\
\mathrm{GeV}\end{array}$ & $\mathrm{MC}$ & $\begin{array}{c}\bar{E}_{\text {miss. }} \\
\mathrm{GeV}\end{array}$ & $\begin{array}{c}\sigma_{E} \\
\mathrm{GeV}\end{array}$ \\
\hline$\Delta Q \leq 1$ & 0.109 & 0.444 & & 0.112 & 0.407 \\
$\Delta Q \geq 2$ & 0.133 & 0.465 & & 0.136 & 0.451 \\
\hline
\end{tabular}

Table 4.3: Average value and standard deviation of the missing energy reconstructed in the $D_{s}$ hemisphere. Events with $\Delta Q \leq 1$ and $\Delta Q \geq 2$ have been separated. 
the behaviour of these events we do not expect a problem in having them included in our candidates.
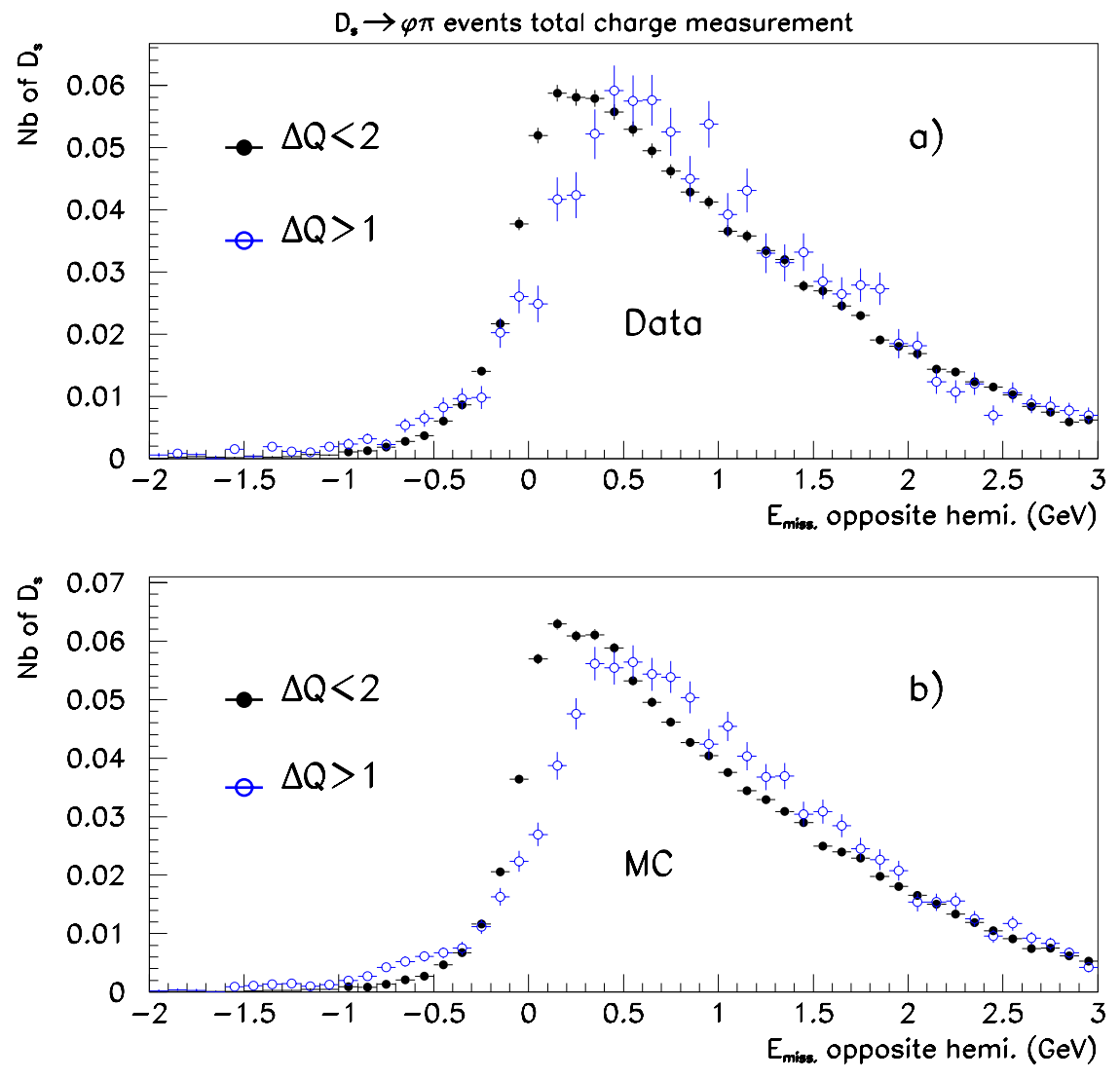

Figure 4.18: Reconstruction of the missing energy in the hemisphere opposite to the $D_{s}$ in data a) and in the simulation b). Events with $\Delta Q \geq 2$ have been separated. Distributions are normalized to unity. 


\subsection{Background adjustements}

The background rate and composition have been determined from dedicated studies of data and simulation. From these comparisons we obtain weights which are applied on simulated events.

The tuning of the background simulation is done separately for the peaking and the nonpeaking components, differenciating also $c \bar{c}, B \bar{B}$ and light quark events. First, we obtain global corrections which are common to peaking and non-peaking components and which differ for $c \bar{c}$ and $B \bar{B}$ events. These corrections are for shape variables used in the Fisher discriminants as $R_{2}$, the total particle multiplicity and, for $c \bar{c}$ events, the fraction of the beam energy transferred to the charm meson.

Secondly, we differenciate variables which differ for peaking and non-peaking background components. In particular, we measure the production of $\phi$ mesons in $B \bar{B}$ and $c \bar{c}$ events and also the rate and kinematic characteristics of charged kaons accompanying a $D$ meson.

Having done these corrections, the forward-backward asymmetry and the event rate measured in data and simulation are compared, outside the $\phi$ region. From these results we evaluate uncertainties on background estimates.

\subsubsection{Fisher variables for the $B \bar{B}$ background}

The control of the $B \bar{B}$ background is done using on-peak events and an off-peak sample of $34.1 \mathrm{fb}^{-1}$. The two samples have been processed through the CharmSL skim and through the same reconstruction algorithm used to reconstruct $D_{s}^{+} \rightarrow \phi e^{+} \nu_{e}$ events. The same cuts are applied except the cuts on the Fisher discriminants. An example of comparison between on-peak and off-peak distributions is shown on Figure 4.19 for $R_{2}$.

Normalizing on-peak data to the luminosity of off-peak data, we subtract the distributions obtained with off-peak data from the on-peak data to keep only the contribution of $B \bar{B}$ events. Then we compare this distribution with the one obtained using the $B \bar{B}$ simulation.

The second Fox Wolfram moment and the multiplicity distributions are shown on Figure 4.20. The difference between data and $\mathrm{MC}$ is corrected defining a weight as function of $R_{2}$ and of the total particle multiplicity. Corrected distributions are shown in Figure 4.21. 


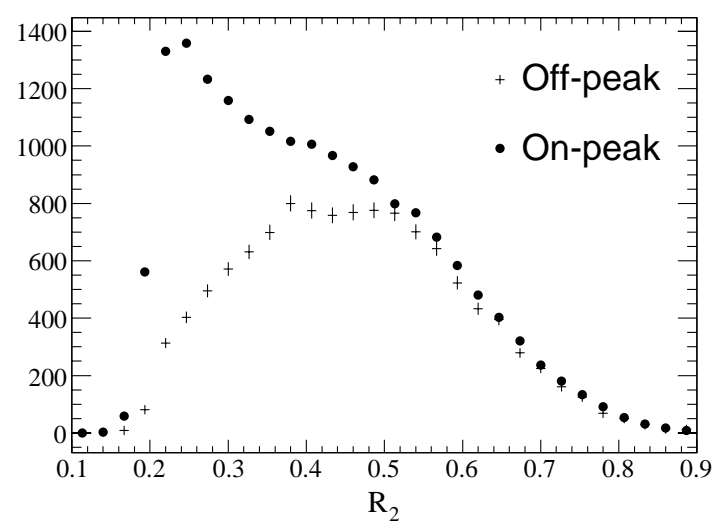

Figure 4.19: Distribution of $R_{2}$ obtained in on-peak and off-peak data events. The on-peak distribution is normalized to the off-peak luminosity.
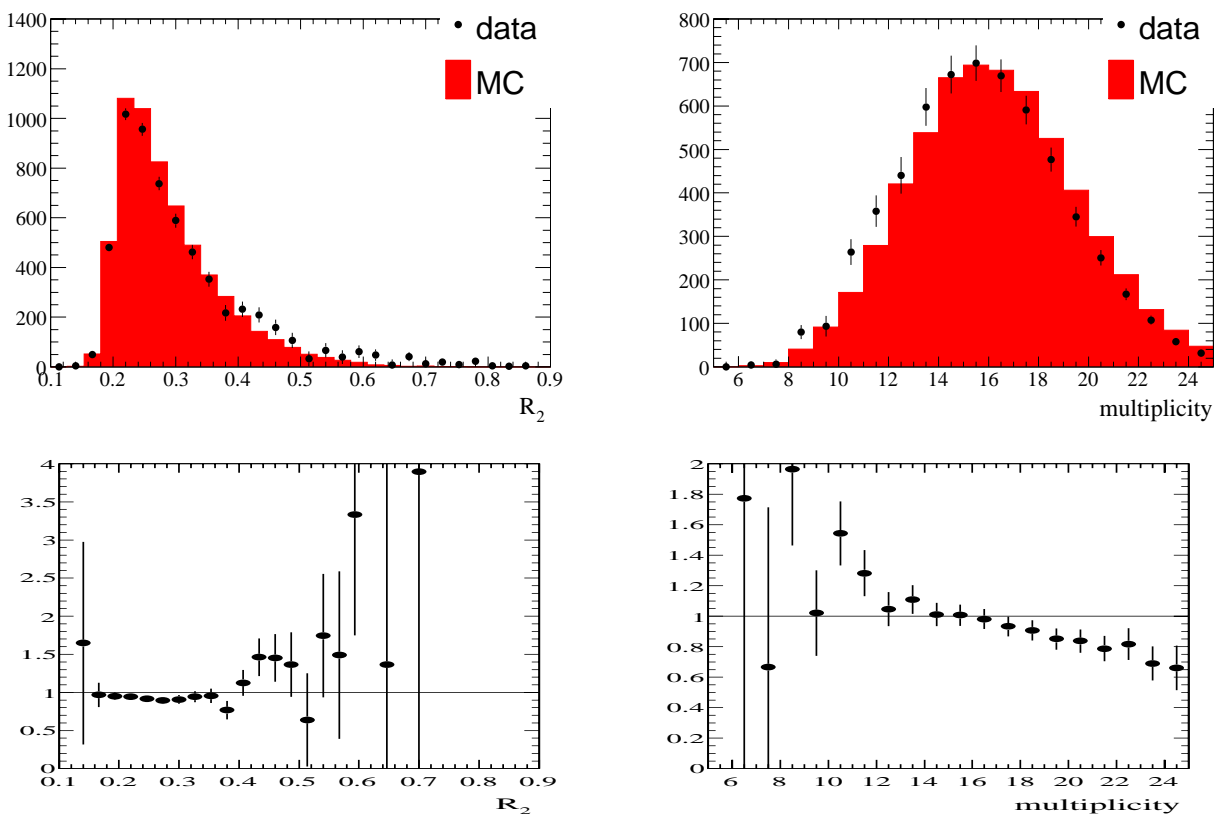

Figure 4.20: Data/MC comparison for $R_{2}$ and the multiplicity. 

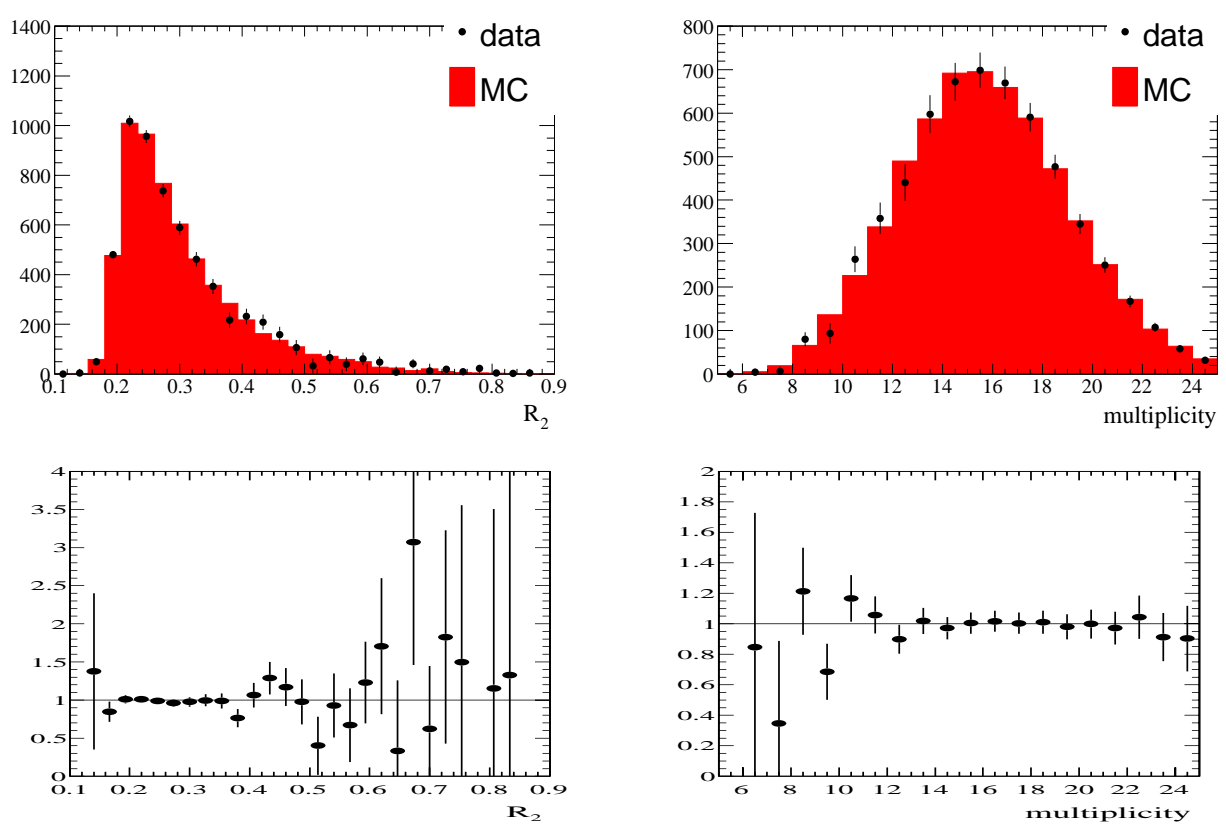

Figure 4.21: Data/MC comparison for $R_{2}$ and the multiplicity after correction.

\subsubsection{Fisher variables for the $c \bar{c}$ background}

As for the signal, the simulated charm background of the $D_{s}^{+} \rightarrow \phi e^{+} \nu_{e}$ decay can be affected by the not-perfect modeling of the c-quark fragmentation process.

For $c \bar{c}$ background events containing a $D_{s}^{+}$in the event hemisphere, we can use the correction as function of the $D_{s}^{+}$momentum determined in Section 4.1.1, and additional corrections on the event shape variables. For other $c \bar{c}$ background events, containing a charged or neutral $D$, we have determined a correction using $D^{0} \rightarrow K^{-} \pi^{+}$events, considering separately cases in which the $D^{0}$ comes from a $D^{*+}$.

To select these events, charged kaon candidates are combined with a pion of opposite charge and the two tracks have to originate from a common vertex with a probability higher than $10^{-3}$. The $D^{0}$ signal region corresponds to events with $m(K \pi) \in[1.837,1.893] \mathrm{GeV} / \mathrm{c}^{2}$. Events in the sidebands, with $m(K \pi) \in[1.774,1.830]$ or $[1.900,1.956]$ are used to obtain the distribution of a given variable corresponding to combinatorial background candidates. $D^{*+}$ candidates are obtained by adding to the $D^{0}$ a pion of charge opposite to the kaon and forming a vertex compatible with the beam spot position with a probability higher than $10^{-3}$. The $K \pi$ mass is selected in the $D^{0}$ signal region and the $D^{*+}$ signal region corresponds to the interval $m\left(D^{0} \pi\right)-m\left(D^{0}\right) \in[0.1448,0.1460] \mathrm{GeV} / \mathrm{c}^{2}$. To evaluate distributions from background events we use candidates between 0.15 and $0.20 \mathrm{GeV} / \mathrm{c}^{2}$. All displayed 
distributions have been subtracted for combinatorial background contributions.

Distributions of the fraction of the beam energy taken by the $D^{*+}\left(x_{D^{*}}\right)$, of $R_{2}$ (also called $h 2 h 0$ ) and of the total particle multiplicity (charged + neutrals) in the event have been compared in Figure 4.22 for data and simulation using off-peak events. A weight, applied on simulated events, is defined as a function of $x_{D^{*}}, R_{2}$ and the total particle multiplicity. The distributions after corrections are shown on Figure 4.23.

The same comparisons have been performed for events containing a $D^{0}$ reconstructed in the $K^{-} \pi^{+}$decay channel in Figures 4.24 and 4.25. It is found that the corresponding corrections are similar as those obtained using the $D^{*+}$ sample. 

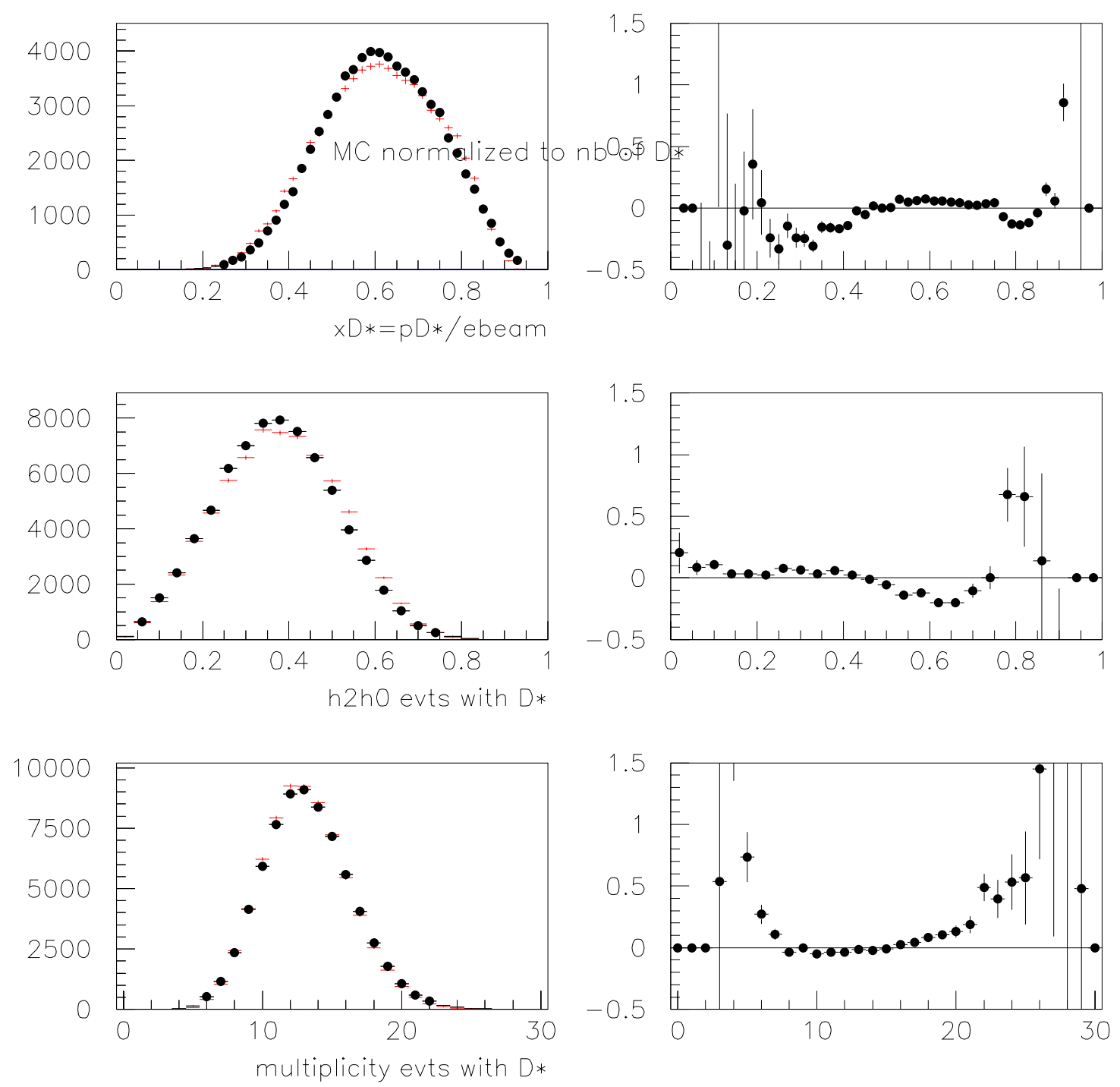

Figure 4.22: Distributions of $x_{D^{*}}, R_{2}$ and the total particle multiplicity in events with a reconstructed $D^{*+}$ obtained in off-peak data (points in black) and in the simulation (red). These distributions have been normalized to the same number of reconstructed $D^{*+}$. Plots on the right gives the ratio $($ data $-M C) / M C$. 

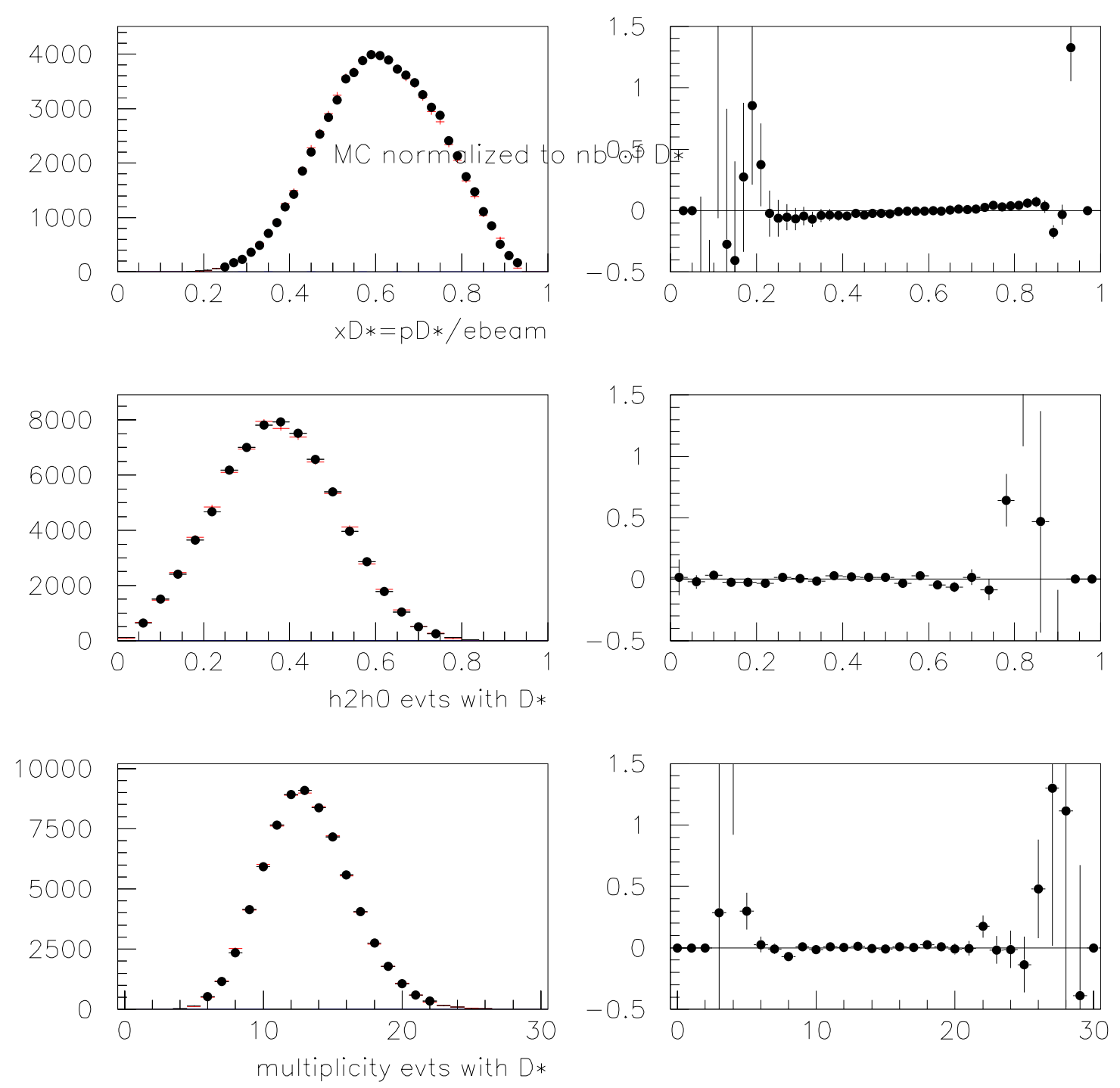

Figure 4.23: Distributions of $x_{D^{*}}, R_{2}(h 2 h 0)$ and the total particle multiplicity in events with a reconstructed $D^{*+}$ obtained in off-peak data (points in black) and in the simulation (red) after applying corrections to simulated events. 

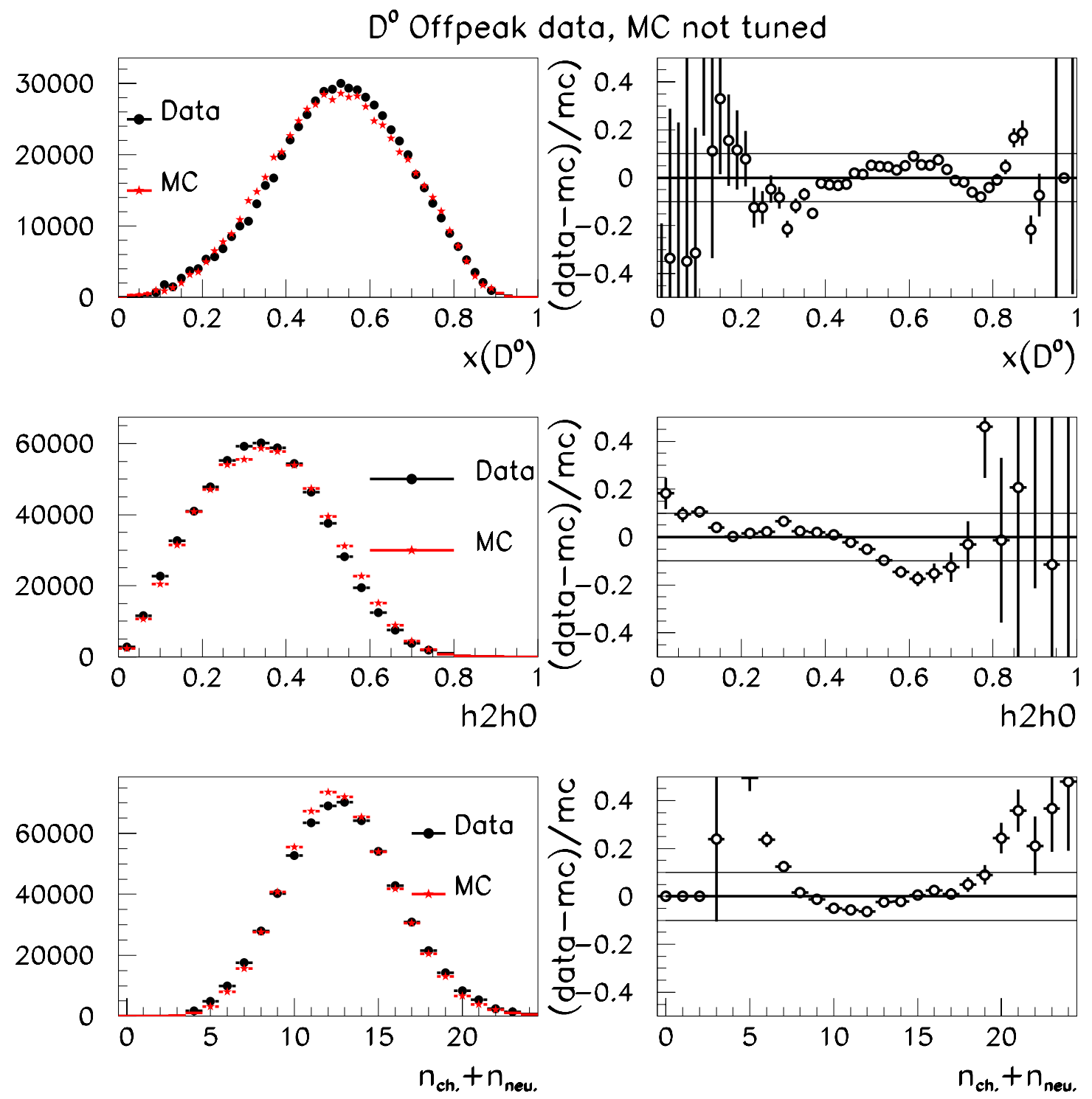

Figure 4.24: Distributions of $x_{D^{0}}, R_{2}(h 2 h 0)$ and the total particle multiplicity in events with a reconstructed $D^{0}$ obtained in off-peak data (points in black) and in the simulation (red). 

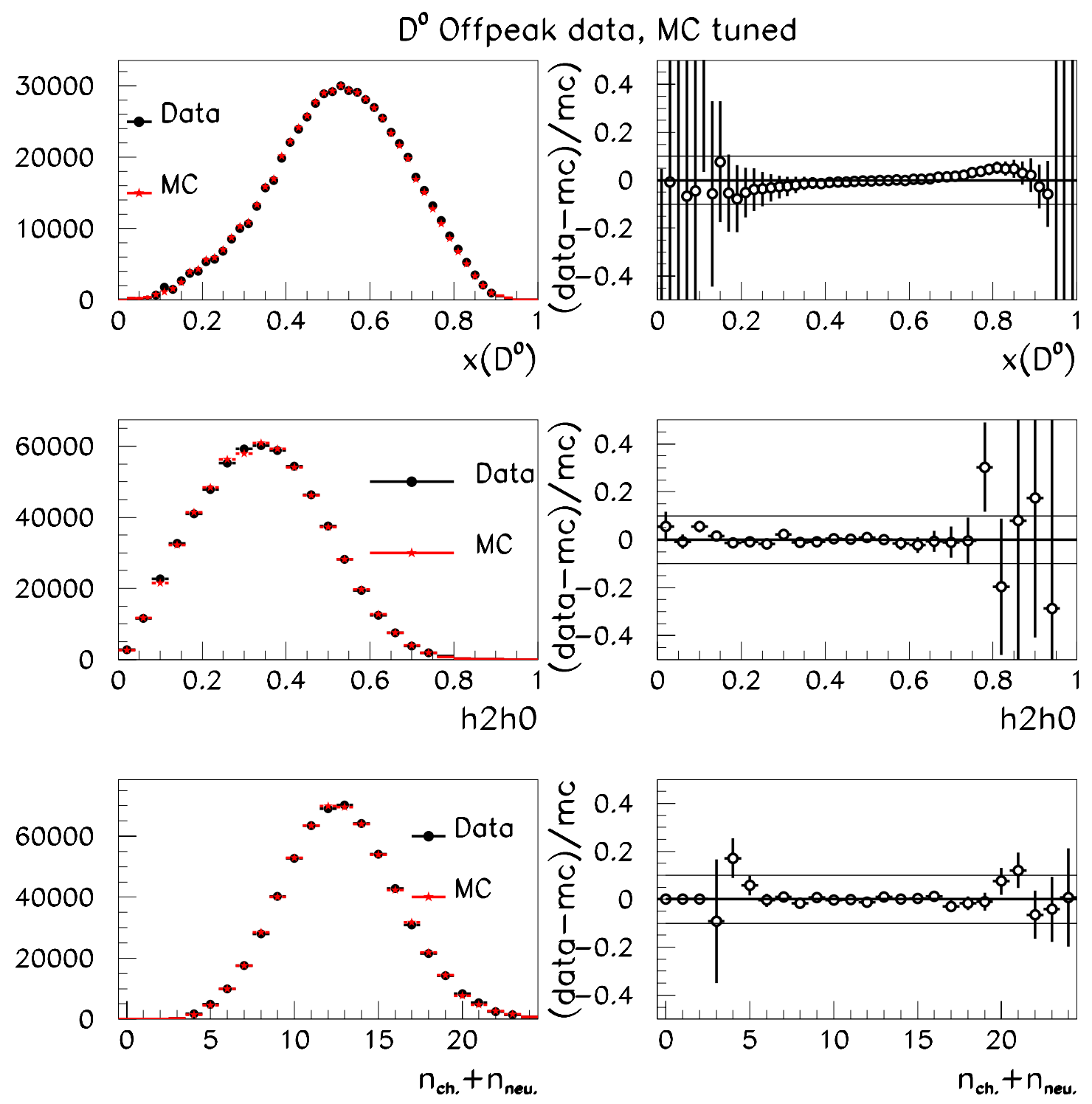

Figure 4.25: Distributions of $x_{D^{0}}, R_{2}(h 2 h 0)$ and the total particle multiplicity in events with a reconstructed $D^{0}$ obtained in off-peak data (points in black) and in the simulation (red) after applying corrections to simulated events. 


\subsection{3 $K^{ \pm}$production in charm events}

As we have explained in Section 3.4.5, the non-peaking $c \bar{c}$ background comes mainly from events in which a pair $e K$ is taken from a $D$ meson decay whereas the other $K$ originates from fragmentation.

To control this background we have measured the production of $K^{ \pm}$mesons in continuum $e^{+} e^{-} \rightarrow c \bar{c}$ events where a $D^{*+}$, a $D^{0}$ or a $D_{s}^{+}$is exclusively reconstructed. Kaon production is considered separately for hadrons emitted in the same or in the opposite direction of the reconstructed $c$-hadron. In the $c$-hadron hemisphere, the remaining particles are produced by the $c$-quark fragmentation whereas, in the opposite hemisphere, they contain also decay products of the other $c$-hadron. To evaluate the non-peaking background we are mainly interested in kaon production in the same hemisphere as the $D$ meson. It should be also noted that the three types of charmed hadrons, $D^{*+}, D^{0}$ and $D_{s}^{+}$correspond, respectively, to kaon production in association with a $c \bar{d}$, a $c \bar{u}$ or a $c \bar{s}$ system.

Simulated events have been weighted already as function of $x_{D}, R_{2}$ and the total multiplicity.

In events with a $D^{0}$ or a $D^{*+}$, we keep events only if $x_{D}>0.48$ to remove contributions from $\Upsilon(4 S)$ decays. In events with a $D_{s}$ we require that its momentum is higher than 2.5 $\mathrm{GeV} / \mathrm{c}$.

We consider separately candidate kaons of same or opposite charge as the $D$, emitted in the same or in the opposite hemisphere containing the $D$. For a neutral $D^{0}$, we consider that its charge is positive and for a $\bar{D}^{0}$ we take its charge as negative.

$$
\text { - } D^{*+}-K^{ \pm} \text {events: }
$$

The $D^{0}$ is reconstructed in the $K^{-} \pi^{+}$decay channel. We analyze $221327 D^{*+}$ from the $c \bar{c}$ MC and 337886 from data. For comparison between data and MC, results have been normalized to the measured number of $D^{*+}$ in data.

For $c \bar{c}$ events, the expected composition of the kaon samples is given in Figure 4.26 and in Table 4.4. It illustrates the dominance of kaons from fragmentation in the $D$ hemisphere. The excess of kaons with same sign as the $D^{*}$ is expected from the fragmentation process which generates such kaons with a momentum on average higher than for opposite sign kaons.

The rate and momentum distributions of kaon candidates reconstructed in the same or in the opposite hemisphere as the $D^{*+}$ are given in Figure 4.27 and in Table 4.5. 

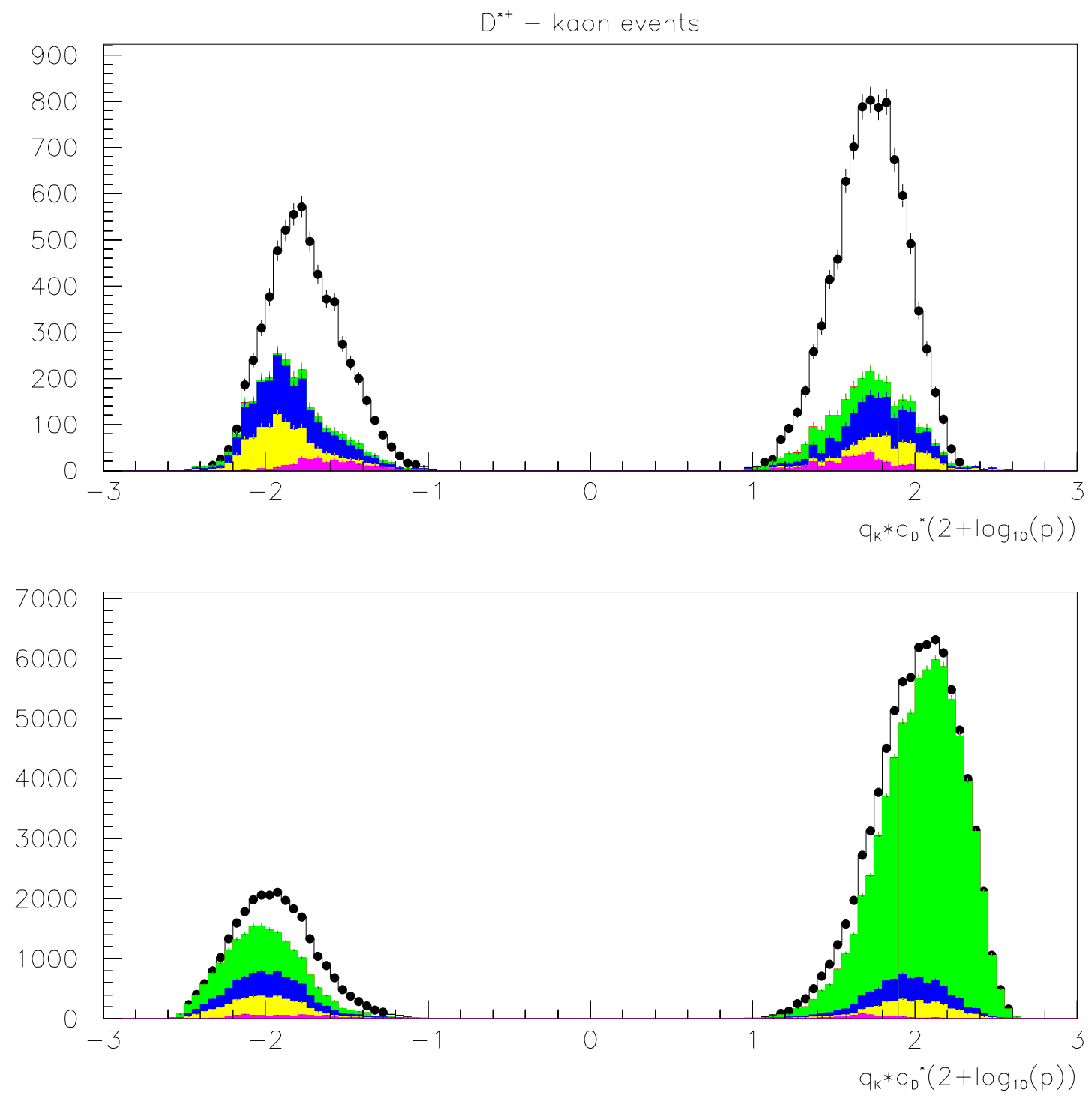

Figure 4.26: Momentum distribution of kaon candidates, depending on their origin. The top(bottom) plot corresponds to kaons in the same(opposite) hemisphere(s) as the $D^{*+}$. The distributions, from top to bottom contain: kaons from fragmentation (unshaded), from charm(green), and three categories of misidentified kaons.

The comparison between data and $\mathrm{MC}$ for candidates in the same hemisphere measures the agreement for kaons from fragmentation. For $K^{-}$the simulation overestimates the rate by $20 \%$ and there are differences in the momentum and angular distributions. For $K^{+}$the agreement is much better. 


\begin{tabular}{|ccc|ccc|}
\hline \multicolumn{2}{|c|}{ same hemisphere } & \multicolumn{4}{c|}{ oppo. hemisph. } \\
$q_{K} \times q_{D^{*}}<0$ & & & $q_{K} \times q_{D^{*}}<0$ & \\
\hline fake & frag. & $D \rightarrow K X$ & fake & frag. & $D \rightarrow K X$ \\
\hline 2310 & 3724 & 215 & 9388 & 8402 & 9512 \\
\hline$q_{K} \times q_{D^{*}}>0$ & & & $q_{K} \times q_{D^{*}}>0$ & & \\
\hline fake & frag. & $D \rightarrow K X$ & fake & frag. & $D \rightarrow K X$ \\
\hline 1818 & 6677 & 703 & 8656 & 9471 & 66322 \\
\hline
\end{tabular}

Table 4.4: Expected numbers of kaon candidates accompanying a $D^{*+}$ obtained from simulation (using 221327 $\mathrm{D}^{*+}$ ).

\begin{tabular}{|c|cc|cc|}
\hline & \multicolumn{2}{|c|}{ same hemisph. } & \multicolumn{2}{c|}{ oppo. hemisph. } \\
& $q_{K} \times q_{D^{*}}<0$ & $q_{K} \times q_{D^{*}}>0$ & $q_{K} \times q_{D^{*}}<0$ & $q_{K} \times q_{D^{*}}>0$ \\
\hline Data & 6497 & 13176 & 36825 & 1178711 \\
MC & 7886 & 12796 & 34993 & 123334 \\
\hline Data/MC & $0.82 \pm 0.02$ & $1.03 \pm 0.02$ & $1.05 \pm 0.01$ & $0.956 \pm 0.005$ \\
\hline
\end{tabular}

Table 4.5: Comparison of kaon production in the same and in the opposite hemisphere as the $D^{*+}$, between simulated and data events.

- $D^{0}-K^{ \pm}$events:

The $D^{0}$ is reconstructed in the $K^{-} \pi^{+}$decay channel, $D^{0}$ which come from the decay of a resonance as $D^{*(+, 0)}$ or $D^{* *}$ are not distinguished.

We have analyzed $614152 D^{0}$ from the $c \bar{c}$ MC and 931918 from data. For comparison between data and simulation, the plots are normalized to the measured number of $D^{0}$ in data. Using simulated $c \bar{c}$ events, the expected composition of the kaon samples is given in Figure 4.28 and in Table 4.6. Same remarks (as those formulated for events with a $D^{*+}$ ) applies for kaons accompanying a $D^{0}$ in its hemisphere.

\begin{tabular}{|ccc|ccc|}
\hline \multicolumn{3}{|c|}{ same hemisph. } & \multicolumn{4}{c|}{ oppo. hemisph. } \\
$q_{K} \times q_{D^{0}}<0$ & & & $q_{K} \times q_{D^{0}}<0$ & \\
\hline fake & frag. & $D \rightarrow K X$ & fake & frag. & $D \rightarrow K X$ \\
\hline 2469.9 & 7396.7 & 533.8 & 14505.0 & 21500.3 & 21559.3 \\
\hline$q_{K} \times q_{D^{0}}>0$ & & & $q_{K} \times q_{D^{0}}>0$ & & \\
\hline fake & frag. & $D \rightarrow K X$ & fake & frag. & $D \rightarrow K X$ \\
\hline 5795.8 & 31235.6 & 3882.0 & 9590.6 & 28085.5 & 159211.0 \\
\hline
\end{tabular}

Table 4.6: Expected numbers of kaon candidates accompanying a $D^{0}$ (from $614152 D^{0}$ ).

The rate and momentum distributions of kaon candidates reconstructed in the same or in the opposite hemisphere as the $D^{0}$ are given in Figure 4.29 and in Table 4.7. 

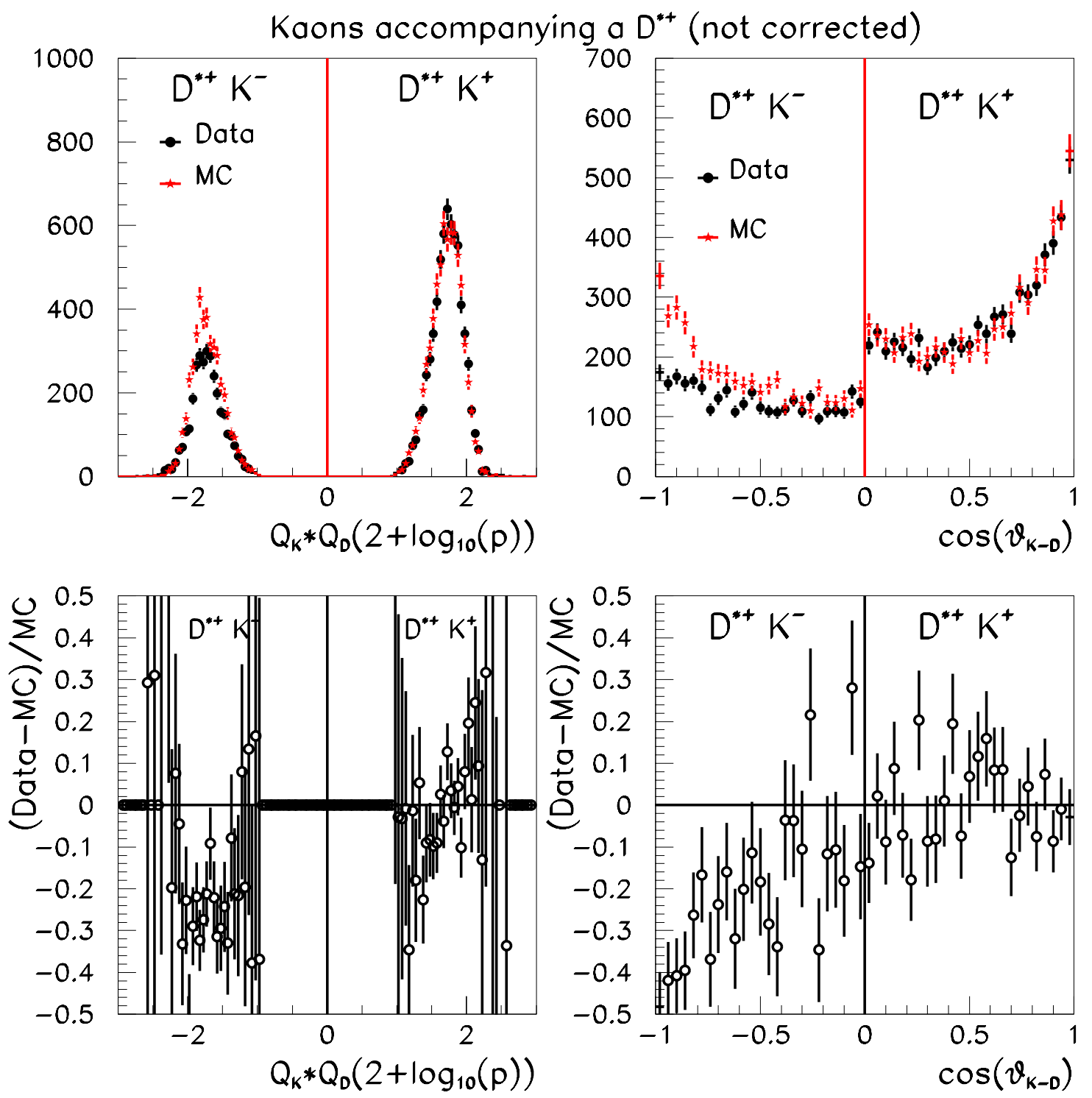

Figure 4.27: Comparison between the measured momentum and angular distributions of kaons in data (black points) and in $M C$ (red histogram) in events with a reconstructed $D^{*+}$ in the same event hemisphere. Lower plots correspond to the relative difference between data and the simulation.

The comparison between data and $\mathrm{MC}$ for candidates in the same hemisphere tests the agreement for kaons from fragmentation. The simulation overestimates by $25 \%$ the fraction of $K^{-}$and underestimates by $10 \%$ the fraction of $K^{+}$. For $K^{+}$, the momentum distribution of the kaon is also rather different in data and in the simulation. We determine a correction 
$D^{0}-$ kaon events
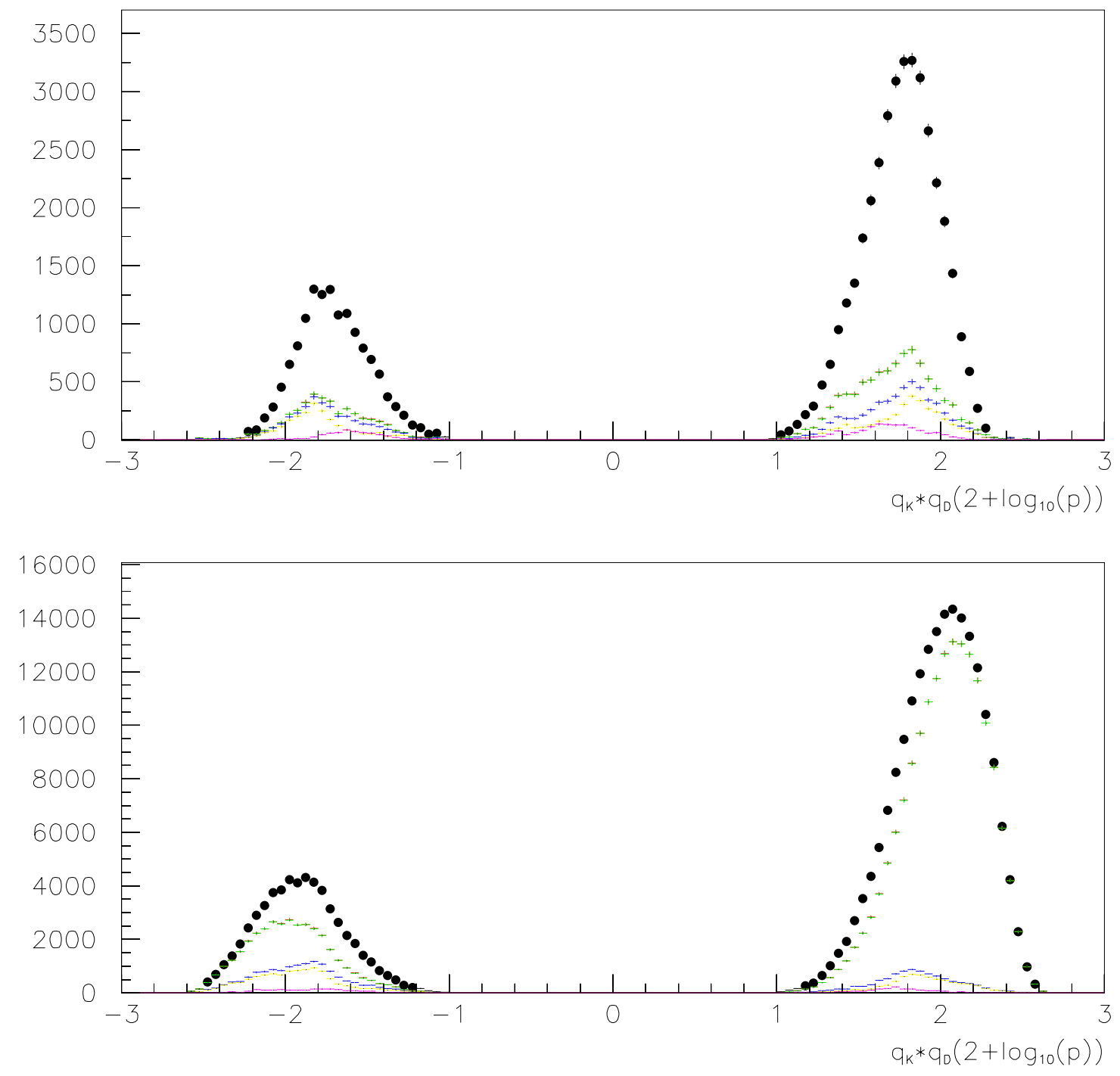

Figure 4.28: Momentum distribution of kaon candidates accompanying 614152 $D^{0}$, depending on their origin. The top(bottom) plot corresponds to kaons in the same(opposite) hemisphere(s) as the $D^{0}$. The distributions, from top to bottom contain: kaons from fragmentation (unshaded), from charm(green), and three categories of misidentified kaons.

which depends on the momentum and on the direction of the kaon from fragmentation such that data and simulation are in agreement.

The corrections which are applied to the simulation and which depend on $x_{D^{0}}, R_{2}$ and the particle multiplicity (see Section 4.3.2), have been obtained comparing events spanning 

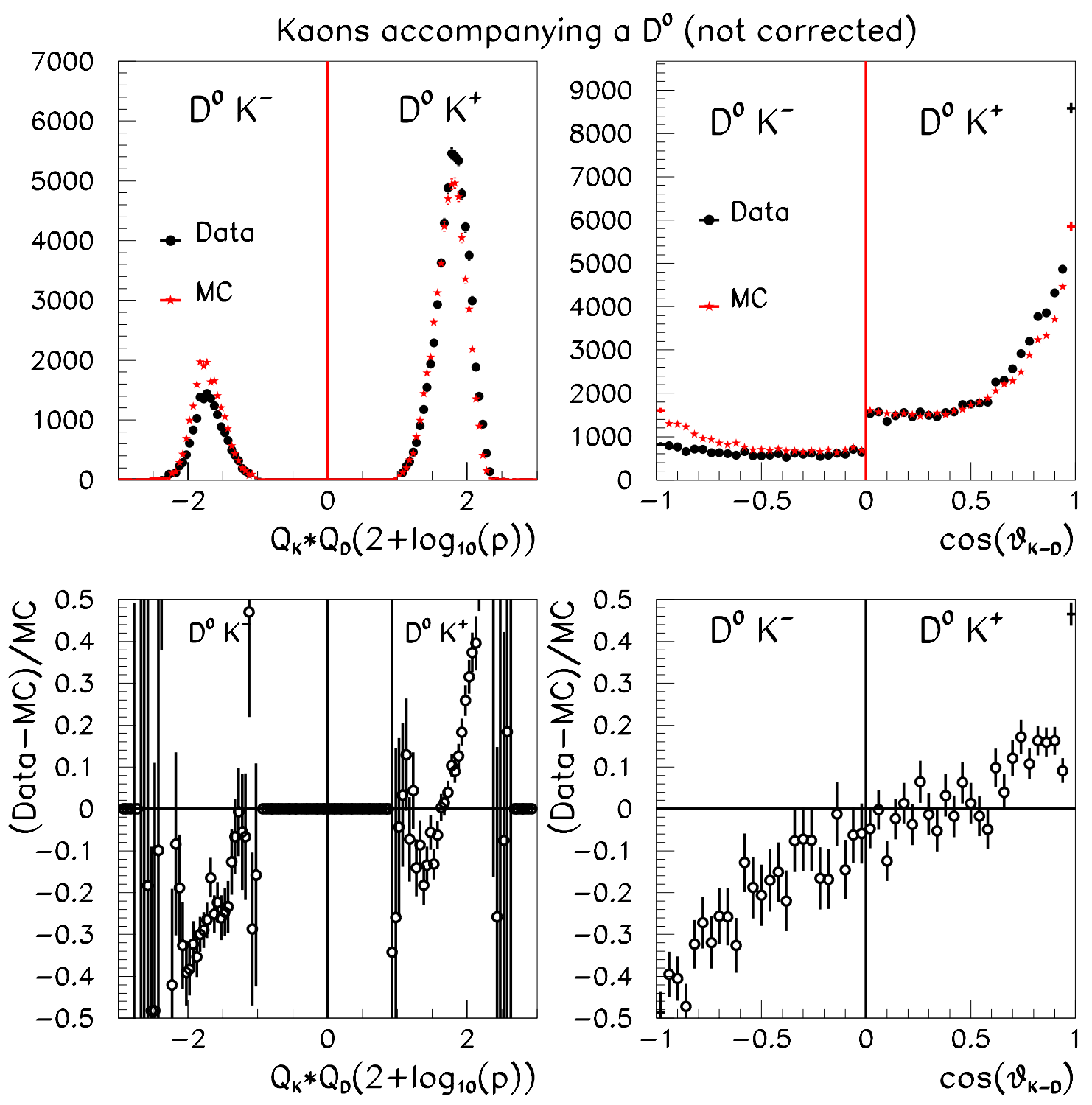

Figure 4.29: Comparison between the measured momentum and angular distributions of kaons in data (black points) and in $M C$ (red histogram) in events with a reconstructed $D^{0}$ in the same event hemisphere. Lower plots correspond to the relative difference between data and the simulation.

the whole range in $x_{D^{0}}$. To study the production of kaons accompanying a $D^{0}$ we select events with $x_{D^{0}}>0.48$, because we use on-peak events that contain also $D^{0}$ from $B$ mesons. It is thus possible that the corrections are not perfect when applied to this sample. This is illustrated in Figure 4.30 which compares data and the simulation, the latter is corrected 


\begin{tabular}{|c|cc|cc|}
\hline & \multicolumn{3}{|c|}{ same hemisph. } & \multicolumn{2}{c|}{ oppo. hemisph. } \\
& $q_{K} \times q_{D^{0}}<0$ & $q_{K} \times q_{D^{0}}>0$ & $q_{K} \times q_{D^{0}}<0$ & $q_{K} \times q_{D^{0}}>0$ \\
\hline Data & 15808.0 & 62243.5 & 90100.5 & 288745.0 \\
MC & 21108.4 & 56467.4 & 87349.2 & 298759 \\
\hline Data/MC & $0.75 \pm 0.01$ & $1.10 \pm 0.01$ & $1.03 \pm 0.01$ & $0.97 \pm 0.01$ \\
\hline
\end{tabular}

Table 4.7: Comparison of kaon production in the same and in the opposite hemisphere as the $D^{0}$, between simulated and real events. A positive value for $q_{D^{0}}$ corresponds to a $D^{0}$ meson and a negative value to a $\bar{D}^{0}$.

using weights determined without a cut on $x_{D^{0}}$. There are differences at the $\pm 10 \%$ level between data and the simulation in the tails of $R_{2}$ and multiplicity distributions. These effects have been included when evaluating systematics.

- $D_{s}^{+}-K^{ \pm}$events:

To compare kaon production in data and simulated events, MC samples are normalized to the number of reconstructed $D_{s}^{+}$mesons in data (58718 using Run2 statistics) ${ }^{1}$. Based on simulated $c \bar{c}$ events, the expected composition of the kaon samples is given in Table 4.8.

\begin{tabular}{|ccc|ccc|}
\hline \multicolumn{2}{|c|}{ same hemisph. } & \multicolumn{3}{c|}{ oppo. hemispere. } \\
$q_{K} \times q_{D_{s}}<0$ & & & $q_{K} \times q_{D_{s}}<0$ & \\
\hline fake & frag. & $D \rightarrow K X$ & fake & frag. & $D \rightarrow K X$ \\
\hline 969 & 23237 & 186 & 2690 & 18487 & 4927 \\
\hline$q_{K} \times q_{D_{s}}>0$ & & & $q_{K} \times q_{D_{s}}>0$ & & \\
\hline fake & frag. & $D \rightarrow K X$ & fake & frag. & $D \rightarrow K X$ \\
\hline 430 & 2722 & 591 & 2313 & 5318 & 34987 \\
\hline
\end{tabular}

Table 4.8: Expected numbers of kaon candidates accompanying a $D_{s}^{+}$(from $96858 D_{s}^{+}$).

The rate and momentum distributions of kaon candidates reconstructed in the same or in the opposite hemisphere as the $D_{s}^{+}$are given in Figure 4.31 and in Table 4.9. In the opposite hemisphere, the ratio $D a t a / M C$ is quite in agreement with the ratio determined in $D^{(0, *+)}$ events. One has to note that the composition of the kaon sample in the same hemisphere is different in $D^{*+}$ and in $D_{s}^{+}$events. Especially, the fraction of events with an accompanying kaon from fragmentation is higher in $D_{s}^{+}$events when $q_{K} \times q_{D_{s}}<0$. This comes from the presence of strangeness in the associated $c$-meson.

\footnotetext{
${ }^{1}$ Run1, Run2 and Run4 have been studied separately and results have been found to be compatible, that is why we just quote numbers from Run2
} 

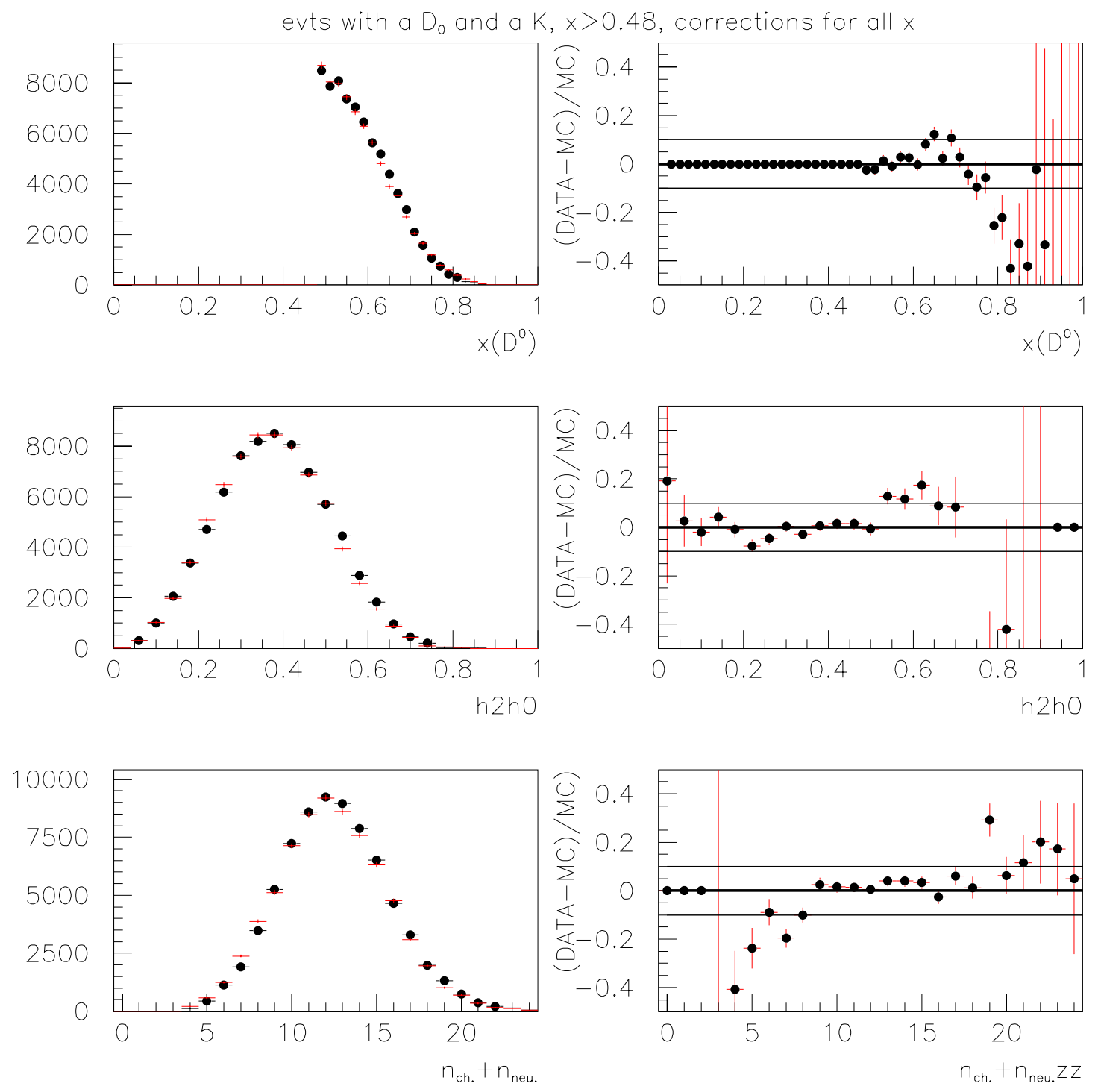

Figure 4.30: Distributions of $x_{D^{0}}, R_{2}$ and the total particle multiplicity in events with a reconstructed $D^{0}$ and a charged kaon in the same hemisphere, selected for $x_{D^{0}}>0.48$ after having applied corrections, on simulated events, defined using events with no selection on $x_{D^{0}}$. Data points are in black and simulation in red. 

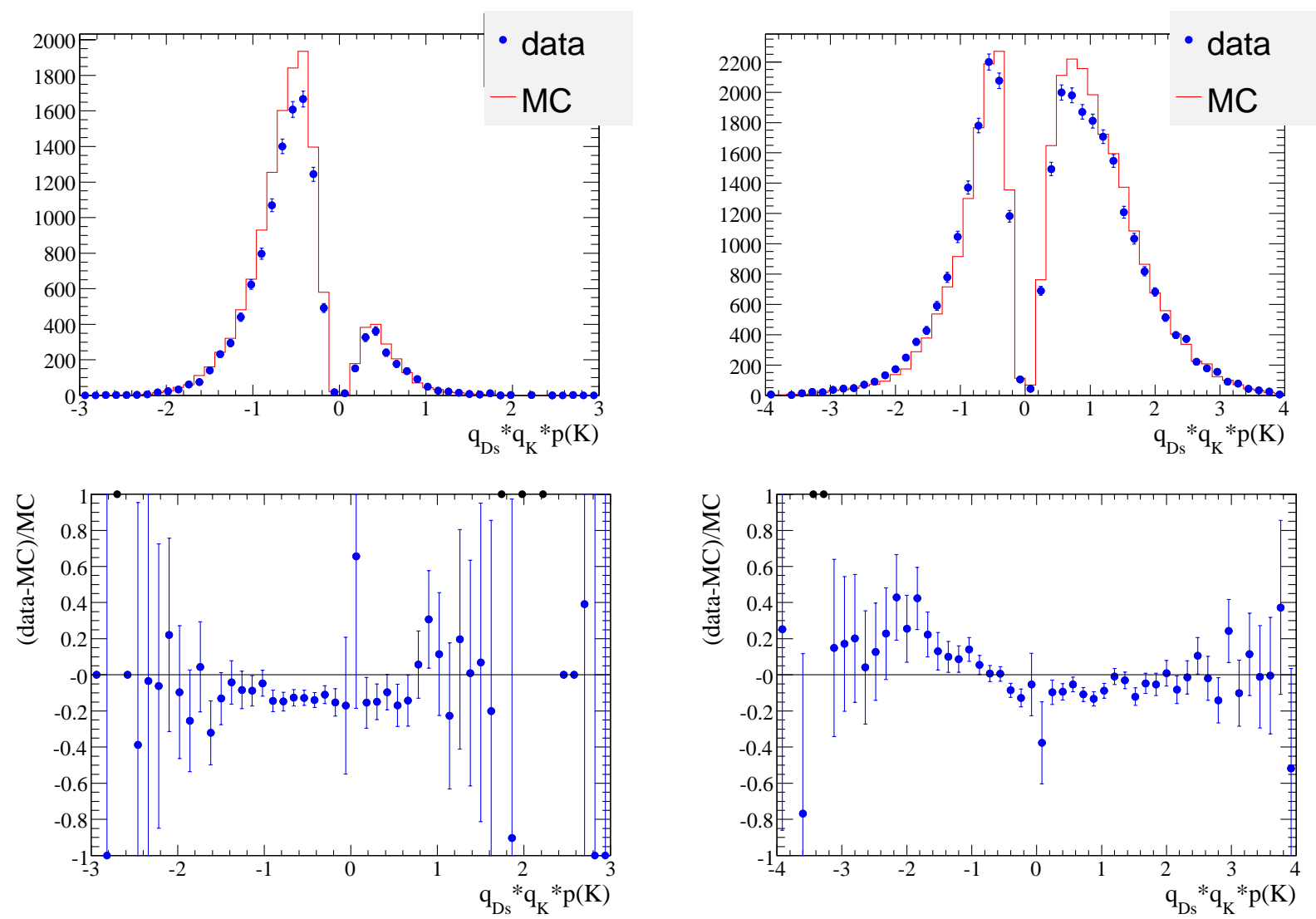

Figure 4.31: Comparison between the measured momentum of Kaons in data(blue) and in $M C$ (red), for Kaons in the $D_{s}^{+}$hemisphere (left) and in the opposite hemisphere (right). The bottom plots are the relative difference between data and $M C$.

\begin{tabular}{|c|cc|cc|}
\hline & \multicolumn{3}{|c|}{ same hemisph. } & \multicolumn{2}{c|}{ oppo. hemisph. } \\
& $q_{K} \times q_{D_{s}}<0$ & $q_{K} \times q_{D_{s}}>0$ & $q_{K} \times q_{D_{s}}<0$ & $q_{K} \times q_{D_{s}}>0$ \\
\hline Data & 10251.4 & 1654 & 12822 & 19104 \\
MC & 11693.4 & 1817 & 12535 & 20516 \\
\hline Data/MC & $0.88 \pm 0.02$ & $0.91 \pm 0.04$ & $1.02 \pm 0.02$ & $0.931 \pm 0.011$ \\
\hline
\end{tabular}

Table 4.9: Comparison of kaon production in the same and in the opposite hemisphere as the $D_{s}^{+}$, between simulated and real events. 
- Summary for $D-K^{ \pm}$events:

For kaons from fragmentation emitted in the same hemisphere as the charm meson we have measured differences between data and simulation on the rate, momentum and angular distributions. These differences depend also on the flavour of the accompanying charm meson. Corrections have been defined according to the type of charm meson, and as function of the momentum and angular distribution. These kaons are the main source of non-peaking background in the $D_{s}^{+} \rightarrow \phi e^{+} \nu$ analysis.

\subsubsection{Charm peaking background}

In events with a $D^{*+}$ or a $D_{s}^{+}$candidate, we search for $\phi \rightarrow K^{+} K^{-}$decays. We separate events in which the $\phi$ is emitted in the same or in the opposite event hemisphere which contains the $D$. Sidebands of the $D$ and $\phi$ signal in the corresponding mass distributions are used to subtract combinatorial background contributions. To compare the $\phi$ production in data and simulated events, the samples have been normalized to the same number of reconstructed $D$ mesons.

- $D^{*+} \phi$ events:

The $K^{+} K^{-}$mass distribution for events with a $D^{*+}$ is shown in Figure 4.32. Momentum distributions for $\phi$ mesons produced in the same (positive values) or in the opposite hemisphere (negative values) as the $D^{*+}$ are given in Figure 4.33 .

In the simulation of $e^{+} e^{-} \rightarrow c \bar{c}$ events with a reconstructed $D^{*+}$, the origin of $\phi$ mesons is given in Table 4.10. Most of the $\phi$ mesons present in the same hemisphere as the $D^{*+}$ come from fragmentation, $20 \%$ are expected to originate from the decay of a second $D$-hadron.

\begin{tabular}{|c|c|c|}
\hline Sample & $\begin{array}{c}\phi \text { opposite } \\
\text { hemisphere }\end{array}$ & $\begin{array}{c}\phi \text { same } \\
\text { hemisphere }\end{array}$ \\
\hline$\phi$ from $D_{s}$ & $1888.1(50.5 \%)$ & 16.7 \\
$\phi$ from $D$ & $1485.0(39.7 \%)$ & 22.3 \\
$\phi$ from frag. & $367.5(9.8 \%)$ & 178.0 \\
\hline
\end{tabular}

Table 4.10: Sources of $\phi$ mesons produced with a $D^{*+}$ measured in simulated events.

Numbers of $\phi$ mesons reconstructed in events with a $D^{*+}$ are given in Table 4.11. The simulation has been normalized to the same number of $D^{*+}$ reconstructed in data. 


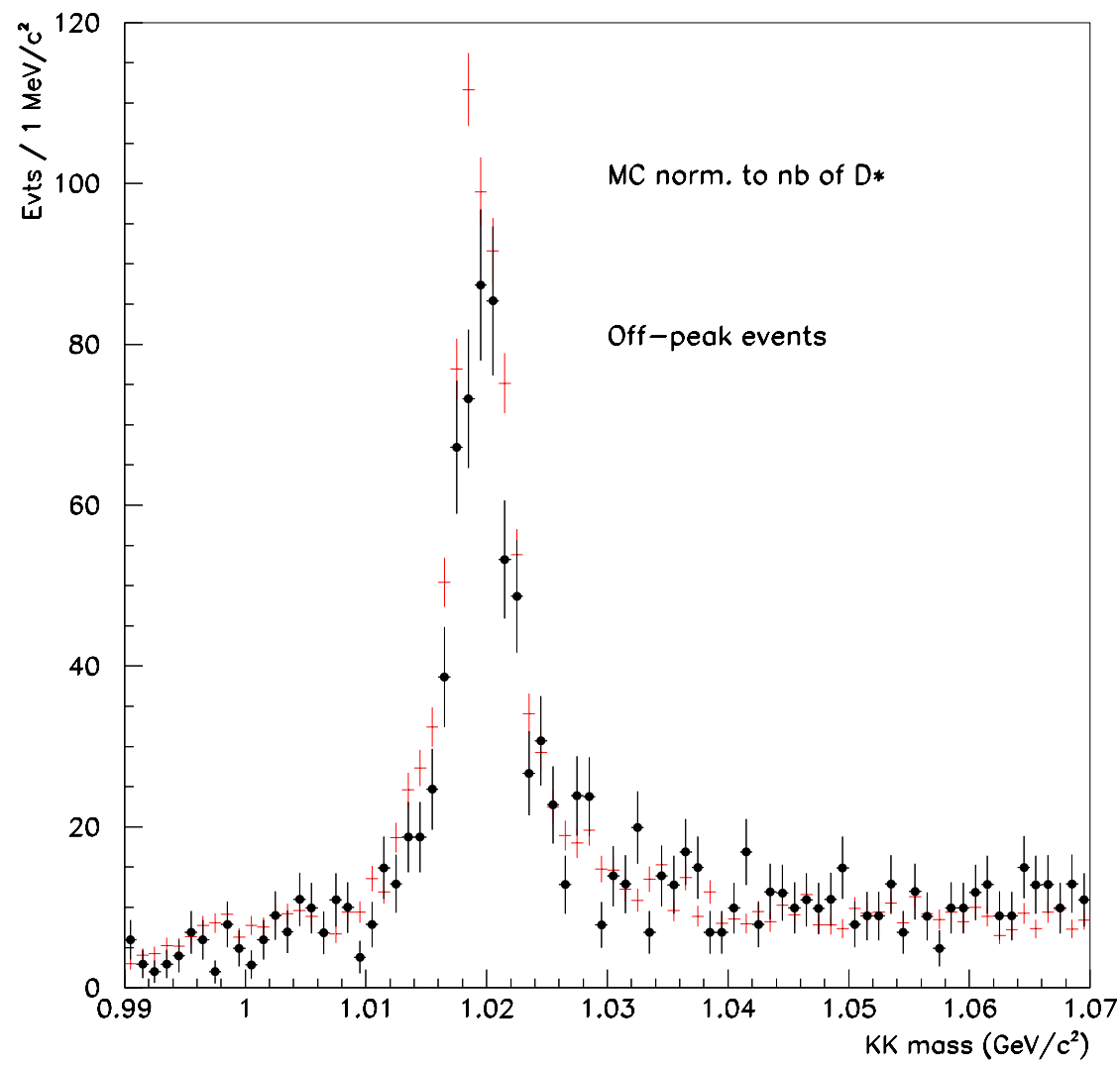

Figure 4.32: $K^{+} K^{-}$mass distribution for events with a reconstructed $D^{*+}$ in Off-peak events. Data (black points) and $M C$ (red) have been normalized to the same number of reconstructed $D^{*+}$ mesons.

\begin{tabular}{|c|c|c|}
\hline Sample & $\begin{array}{c}\phi \text { opposite } \\
\text { hemisphere }\end{array}$ & $\begin{array}{c}\phi \text { same } \\
\text { hemisphere }\end{array}$ \\
\hline \hline On peak $x_{D^{*+}}>0.48$ & & \\
\hline MC $c \bar{c}$ & $2177.7 \pm 47.9$ & $76.3 \pm 11,1$ \\
Data & $1653.8 \pm 51.3$ & $94.9 \pm 13.4$ \\
\hline Data/MC & $0.76 \pm 0.03$ & $1.24 \pm 0.25$ \\
\hline \hline Off peak no cut on $x_{D^{*+}}$ & & \\
\hline MC $c \bar{c}$ & $646.1 \pm 12.8$ & $32.7 \pm 3.5$ \\
Data & $477.6 \pm 27.1$ & $49.2 \pm 9.0$ \\
\hline Data/MC & $0.74 \pm 0.05$ & $1.5 \pm 0.3$ \\
\hline
\end{tabular}

Table 4.11: Comparison between data and simulation for $\phi$ production in events with a $D^{*+}$. 


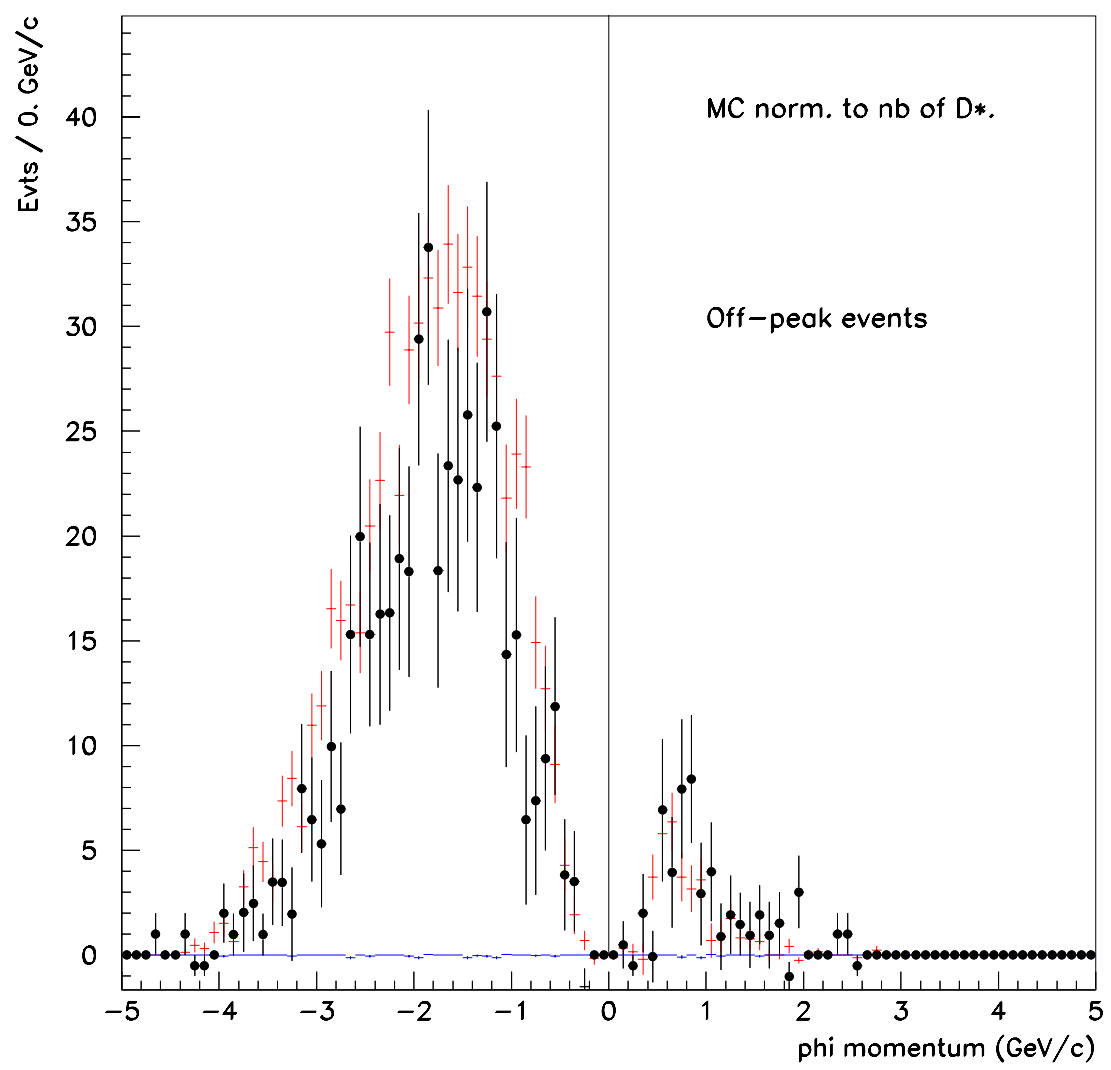

Figure 4.33: Momentum distributions of $\phi$ mesons acccompanying a $D^{*+}$ measured in data (black points) and in the $M C$ (red). Positive values correspond to the situation where the two mesons are produced in the same event hemisphere.

There is a possible excess, in data, of $\phi$ mesons from fragmentation. But given the low statistics, the rate of $\phi$ mesons accompanying a non-strange $D$-meson cannot be verified with an accuracy better than $20 \%$.

We measure a deficit of $\phi$ mesons coming from the inclusive decay $D \rightarrow \phi X$ ( $\phi$ mesons produced in the opposite hemisphere). The ratio of data and MC is rather constant for $\phi$ momenta below $3 \mathrm{GeV} / \mathrm{c}$ and we observe a larger excess in the simulation of $\phi$ production at higher $\phi$ momenta.

- $D_{s}^{+}-\phi$ events:

Using the Run4 statistics, the $K^{+} K^{-}$mass distribution for events with a $D_{s}^{+}$is shown in Figure 4.34. Momentum distributions for $\phi$ mesons produced in the same (positive values) 
or in the opposite hemisphere (negative values) as the $D_{s}^{+}$are given in Figure 4.35. Reconstructed $\phi$ mesons from fragmentation have a higher energy in data than in the simulation.

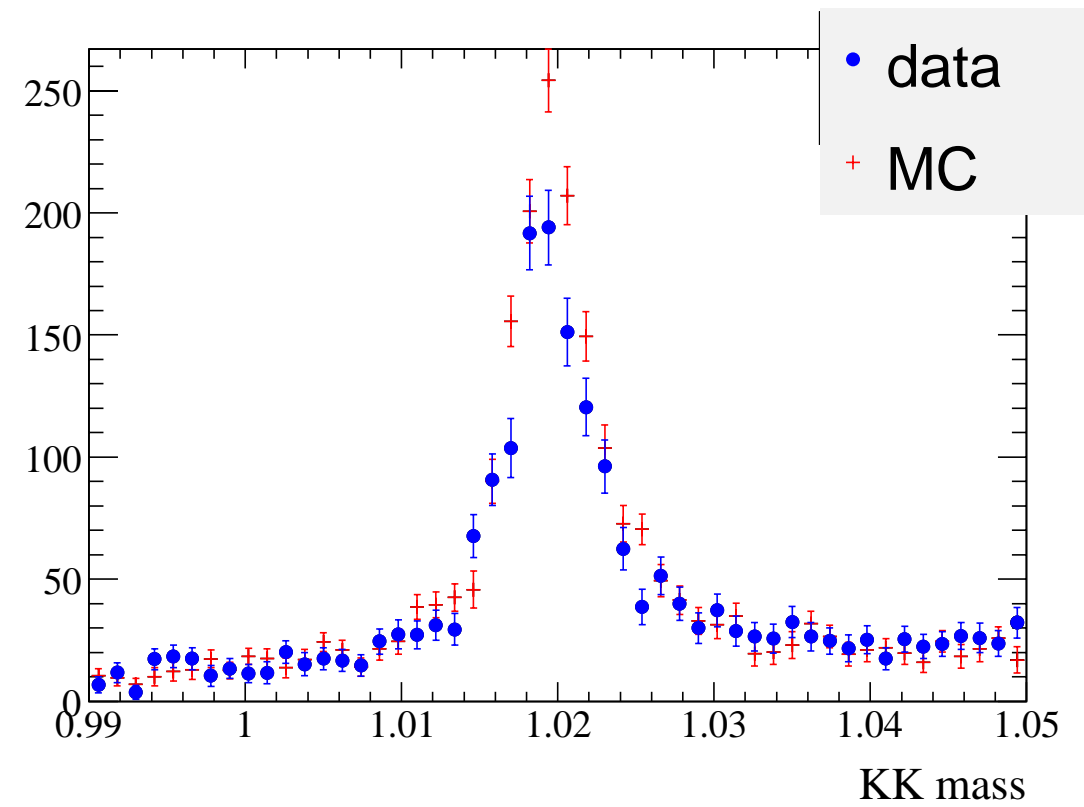

Figure 4.34: $K^{+} K^{-}$mass distribution for events with a reconstructed $D_{s}^{+}$. Data (blue points) and $M C\left(\right.$ red) have been normalized to the same number of reconstructed $D_{s}^{+}$mesons.

In the simulation of $e^{+} e^{-} \rightarrow c \bar{c}$ events with a reconstructed $D_{s}^{+}$, the origin of $\phi$ mesons is given in Table 4.12. Most of the $\phi$ mesons present in the same hemisphere as the $D_{s}^{+}$come from fragmentation. We note also an excess of $\phi$ from fragmentation emitted in the opposite hemisphere as compared with the $D^{*+}$ tag. This is because $\phi$ meson from fragmentation have a low energy and can appear in both hemispheres.

Number of $\phi$ mesons reconstructed in events with a $D_{s}^{+}$are given in Table 4.13, separately for Run1, 2 and 4 (corresponding to 19666, 58718 and $91334 D_{s}$ respectively). We measure a deficit of about $10 \%$ of $\phi$ mesons coming from the inclusive decay $D \rightarrow \phi X$ in data, in the hemisphere opposite to the $D_{s}$ meson.

\begin{tabular}{|c|c|c|}
\hline Sample & $\begin{array}{c}\phi \text { opposite } \\
\text { hemisphere }\end{array}$ & $\begin{array}{c}\phi \text { same } \\
\text { hemisphere }\end{array}$ \\
\hline$\phi$ from $D_{s}$ & $1031(37.5 \%)$ & $17(1.5 \%)$ \\
$\phi$ from $D$ & $791(28.7 \%)$ & $2(0.1 \%)$ \\
$\phi$ from frag. & $931(33.8 \%)$ & $1139(98.4 \%)$ \\
\hline
\end{tabular}

Table 4.12: Sources of $\phi$ mesons produced with a $D_{s}^{+}$measured for simulated events. 


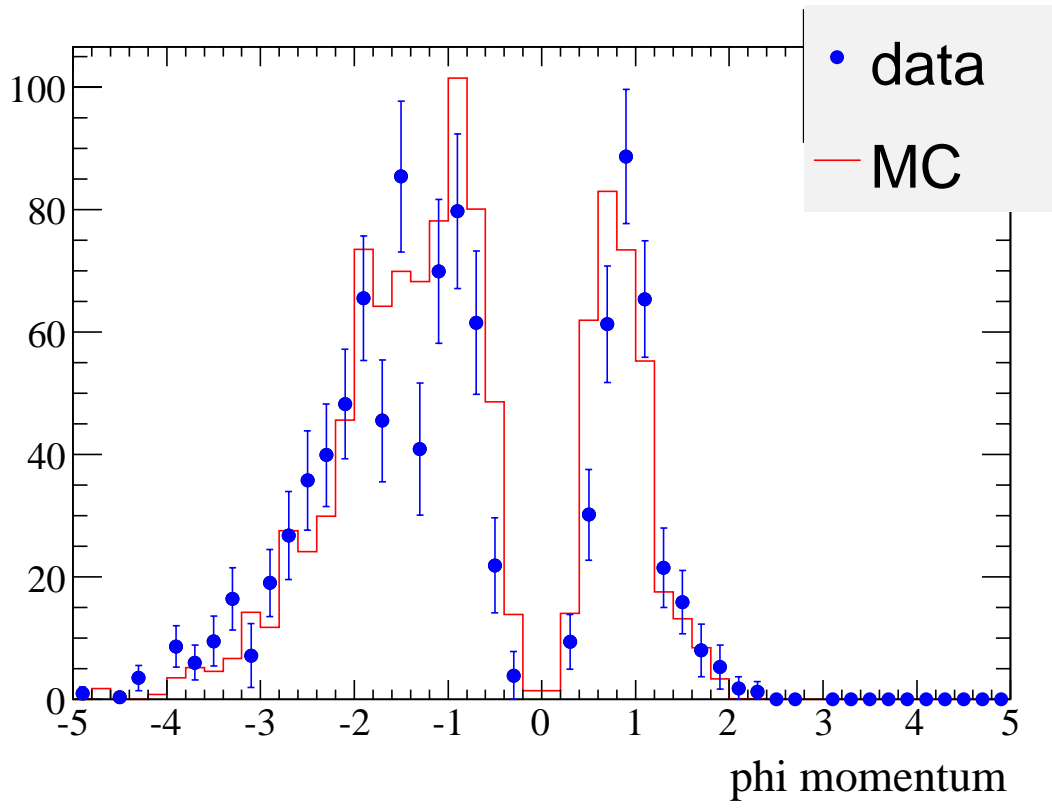

Figure 4.35: Momentum distributions of $\phi$ mesons acccompanying a $D_{s}^{+}$measured in data (blue points) and in the $M C$ (red). Positive values correspond to the situation where the two mesons are produced in the same event hemisphere.

\begin{tabular}{|c|c|c|}
\hline sample & $\begin{array}{c}\phi \text { opposite } \\
\text { hemisphere }\end{array}$ & $\begin{array}{c}\phi \text { same } \\
\text { hemisphere }\end{array}$ \\
\hline \hline Run 1 & & \\
\hline MC $c \bar{c}$ & $172.9 \pm 9.3$ & $78.4 \pm 5.6$ \\
Data & $184.5 \pm 17.3$ & $85.7 \pm 10.8$ \\
\hline Data/MC & $1.07 \pm 0.10$ & $1.09 \pm 0.14$ \\
\hline \hline Run 2 & & \\
\hline MC $c \bar{c}$ & $565.9 \pm 20.3$ & $219.3 \pm 11.8$ \\
Data & $469.5 \pm 30.3$ & $189.7 \pm 18$ \\
\hline Data/MC & $0.83 \pm 0.08$ & $0.86 \pm 0.12$ \\
\hline \hline Run 4 & & \\
\hline MC $c \bar{c}$ & $785.1 \pm 31.5$ & $337.6 \pm 19$ \\
Data & $699.3 \pm 37$ & $308.8 \pm 22$ \\
\hline Data/MC & $0.89 \pm 0.07$ & $0.92 \pm 0.10$ \\
\hline \hline Average & $0.91 \pm 0.05$ & $0.94 \pm 0.07$ \\
\hline
\end{tabular}

Table 4.13: Comparison between data and simulation for $\phi$ production with a $D_{s}^{+}$in Run $1,2,4$. 
- Summary for $D-\phi^{ \pm}$and $D \rightarrow \phi X$ events:

Combining the different results, we obtain that the data/MC agreement for $\phi$ coming from fragmentation has been found compatible with 1, with an accuracy of $20 \%$.

For $\phi$ coming from c-hadrons, we find 0.8 with a $10 \%$ accuracy. These rescaling are applied on the charm peaking background for the $D_{s}^{+} \rightarrow \phi e^{+} \nu$ analysis. 


\subsubsection{The non-peaking background overall rate}

The study of the non-peaking background is done using the wings of the $K^{+} K^{-}$mass distribution of the $D_{s}^{+} \rightarrow \phi e \nu_{e}$ events. This distribution is shown in Figure 4.36 using a logarithmic scale and for a range from $0.98 \mathrm{GeV} / \mathrm{c}^{2}$ to $1.5 \mathrm{GeV} / \mathrm{c}^{2}$. On this plot, all corrections defined previously have been applied, except the one concerning non-peaking $c \bar{c}$ events, determined with $D-K^{ \pm}$events (section 4.3.3). Simulated events are scaled to the data luminosity. One can see a discrepancy between data and simulation, with a mass dependence. This discrepancy is partially explained by the fact that the simulated $\phi$ mass distribution is cut above $1.08 \mathrm{GeV} / \mathrm{c}^{2}$ by the generator. To get rid of this effect, we have generated a sample of non-resonant $D_{s}^{+} \rightarrow K^{+} K^{-} e^{+} \nu_{e}$ events which we reweight to simulate the expected $\phi$ mass Breit-Wigner dependence.
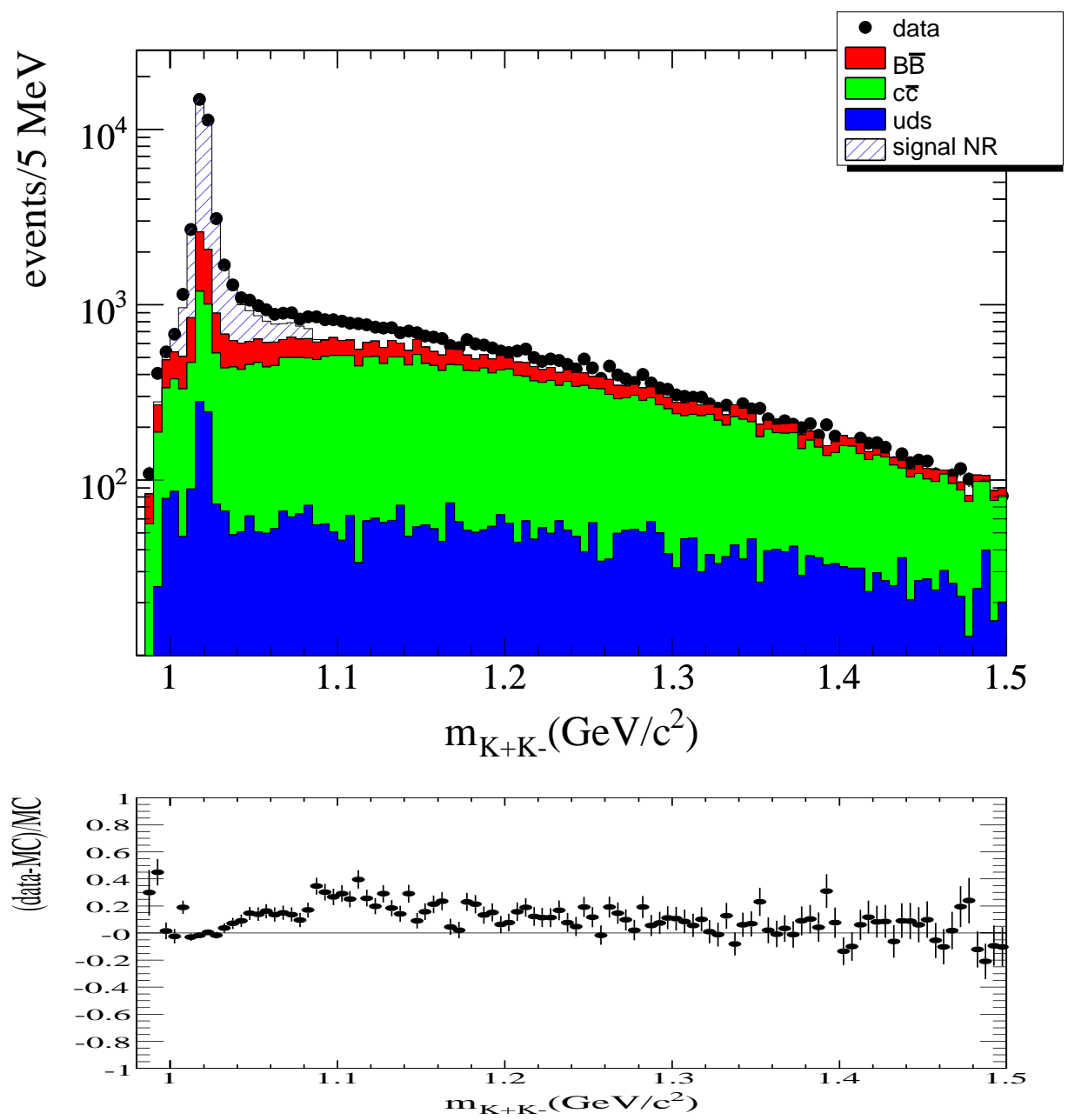

Figure 4.36: Data/MC comparison for $K^{+} K^{-}$mass distribution. The $M C$ is scaled to the data luminosity. The bottom plot shows the ratio $($ data $-M C) / M C$. 
To understand the remaining difference, we have checked the $c \bar{c}$ component and the effect of the corrections defined with $D-K^{ \pm}$events on the continuum $c \bar{c}$ background. The composition of the continuum $c \bar{c}$ background is given in Section 3.4.5. A large fraction of this background involves a kaon from fragmentation and a kaon from a charm meson, thus one can exploit the asymmetry due to the different momentum distributions of the two kaons. For instance, in events with a candidate positron, most of $K^{-}$come from a charm meson decay and $K^{+}$from fragmentation. Consequently, the $K^{+}$has on average less energy than the $K^{-}$and the angle $\theta_{V}$ is closer to $\pi$, so we expect to see a negative asymmetry in $\cos \left(\theta_{V}\right)$. The distributions of the mean value of $\cos \left(\theta_{V}\right)$ as function of the $K^{+} K^{-}$mass for data and $\mathrm{MC}$ are shown on Figure 4.37, with the corresponding ratios. In the same Figure we have also displayed the $K^{+} K^{-}$mass distribution for events weighted by $\cos \left(\theta_{V}\right)$. Only $c \bar{c}$ events are contributing to this asymmetry; there is no asymmetry for $B \bar{B}$ or light quark $q \bar{q}$ events. These distributions are obtained using corrections depending on the fragmentation and on the Fisher variables. If we use, in addition, the corrections that we measure for $D-K$ events, we can see in Figure 4.38 that the agreement between data and simulation is much better.

The last correction applies to light quark $q \bar{q}$ events. On Figure 4.36 one can see a second peak in the $u d s$ simulation at about $1 \mathrm{GeV} / \mathrm{c}^{2}$. It corresponds to the $f_{0} \rightarrow K^{+} K^{-}$which is not correctly modelled. Figure 4.39 left shows the uds background component, with the $f_{0}$ clearly isolated. Replacing the simulated $f_{0}$ by the one generated according to a Flatté distribution (see Eq.(1.40)), one obtains the right plot on Figure 4.39. In this plot, the $f_{0}$ is normalized to the number of $\phi$, using measurements form LEP. The production of $f_{0}$ mesons has been measured at LEP by DELPHI [63] and OPAL [64] collaborations. This is an inclusive measurement in which particles produced in heavy flavour hadron decays are not separated from those created by fragmentation. In anycase the fragmentation mechanism is clearly dominant. The $f_{0} \rightarrow \pi^{+} \pi^{-}$decay channel is used to select the signal and they assume that the branching fraction $B R\left(f_{0} \rightarrow \pi \pi\right)$ is equal to $80 \%$. The particle data group evaluation of this branching fraction is not still available as the dispersion of the different determinations is large. If one takes the values of the $f_{0}$ parameters as measured by BES [23], then one expects that this fraction is indeed $\sim 50 \%$. The production of the $\phi$ meson has been also measured by ALEPH [65], DELPHI [66] and OPAL [64] collaborations, with the $\phi$ reconstructed into $K^{+} K^{-}$. Averaged numbers of $f_{0}$ and $\phi$ mesons measured in a $Z^{0}$ decay are given in Table 4.3.5.

Using the value $\phi \rightarrow K^{+} K^{-}$from the PDG [4] we thus expect the following ratio between 

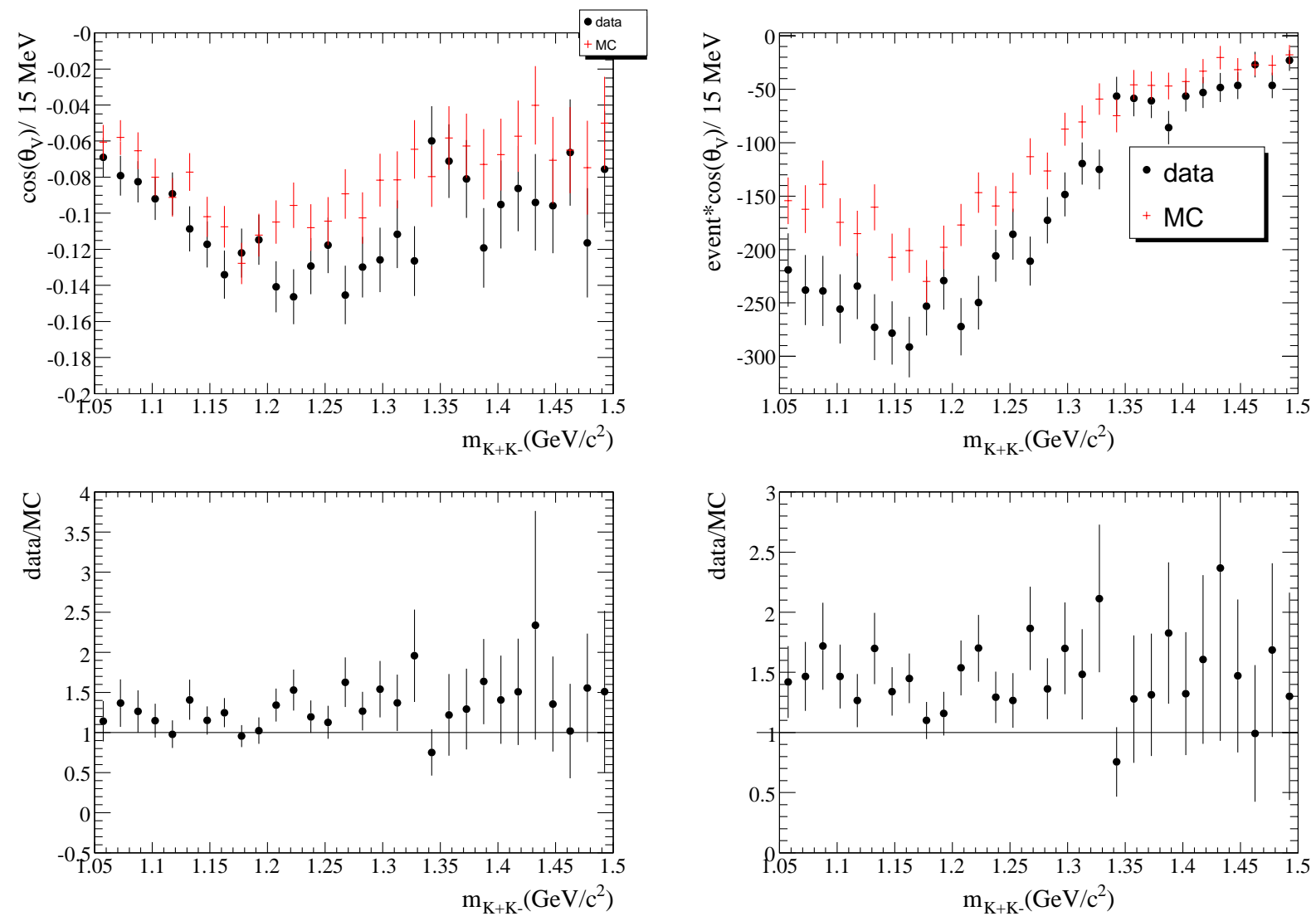

Figure 4.37: Left hand plots: data/MC comparison for the mean value of $\cos \left(\theta_{V}\right)$ per $K^{+} K^{-}$ mass bin and data over $M C$ ratio. Left hand plots $\cos \left(\theta_{V}\right)$ as function of $K^{+} K^{-}$mass, uds and $B \bar{B}$ events have been subtracted from data.

\begin{tabular}{|c|c|c|}
\hline Experiment & $\bar{n}\left(f_{0}\right)$ & $\bar{n}(\phi)$ \\
\hline ALEPH & & $0.122 \pm 0.009$ \\
DELPHI & $0.164 \pm 0.021$ & $0.104 \pm 0.006$ \\
OPAL & $0.141 \pm 0.013$ & $0.091 \pm 0.004$ \\
\hline average & $0.147 \pm 0.011$ & $0.097 \pm 0.007$ \\
\hline
\end{tabular}

Table 4.14: Averaged numbers of $f_{0}$ and $\phi$ mesons measured at LEP in a $Z^{0}$ hadronic decay. The numbers of $f_{0}$ have to be scaled by $0.8 / 0.5$ to take into account present determinations of $f_{0}$ decay parameters. The uncertainty on the average number of $\phi$ mesons has been increased to account for the poor compatibility of individual measurements. 

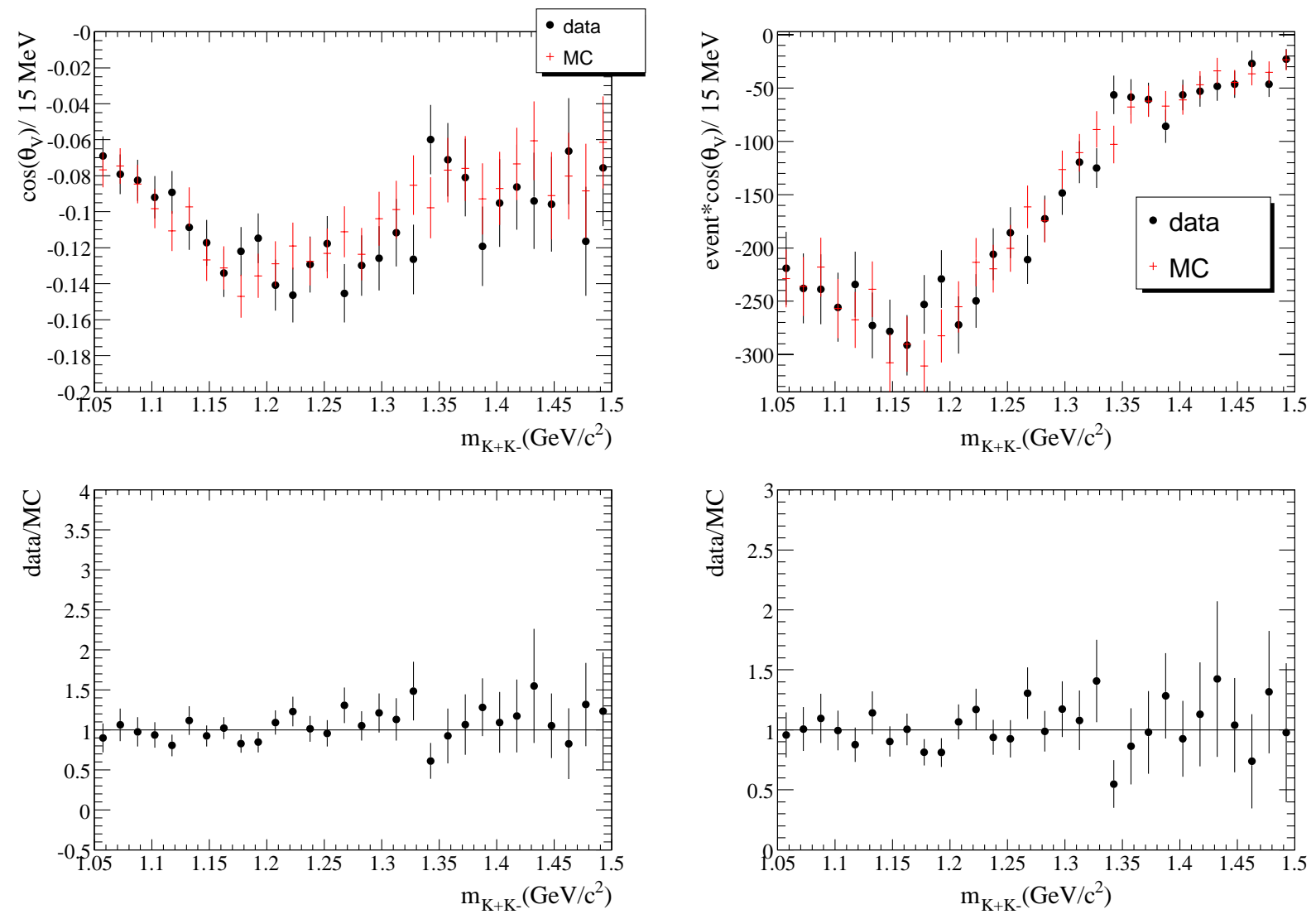

Figure 4.38: Left hand plots: data/MC comparison for the mean value of $\cos \left(\theta_{V}\right)$ per $K^{+} K^{-}$ mass bin and data over $M C$ ratio. Left hand plots: $\cos \left(\theta_{V}\right)$ as function of the $K^{+} K^{-}$mass, uds and $B \bar{B}$ simulated events have been subtracted from data. Corrections for charm nonpeaking background have been applied. 
$f_{0}$ and $\phi$ mesons decaying in the $K^{+} K^{-}$channel:

$$
\frac{n\left(f_{0}\right)}{n(\phi)} \frac{B R\left(f_{0} \rightarrow K^{+} K^{-}\right)}{B R\left(\phi \rightarrow K^{+} K^{-}\right)}=\frac{0.147 \pm 0.011}{0.097 \pm 0.007} \times \frac{0.8}{0.5} \times \frac{0.26}{0.49} \simeq 1.3
$$

In fact this result is rather surprising. The rate of $f_{0}$ seems rather high. From studies of a large variety of particles produced at LEP, it was concluded that the $f_{0}$ does not behave as if it has a dominant component of strange quarks but contains mainly light quarks. This is not in agreement with more recent measurements from BES which show a large coupling of the $f_{0}$ to $K^{+} K^{-}$. Another possibility consists in invoking "a bare mass" (pole of the K-matrix) for the $f_{0}$ of $720 \mathrm{MeV} / \mathrm{c}^{2}$ and to consider that it governs its production rate. In this case a large $s \bar{s}$ component is still allowed [67].

In the following we assume that this fraction is equal to $1.0 \pm 0.5$. The exact value of this ratio has no real effect on the present analysis. As the form factors measurement performed in this analysis is restricted to the mass range between 1.01 and $1.03 \mathrm{GeV} / \mathrm{c}^{2}$, we assume that the behaviour of events with a $f_{0}$ in this range is similar to the one of continuum uds events, and we simply rescale the latter by a factor $1.5 \pm 0.25$ (not including those with a reconstructed $\phi$ meson).
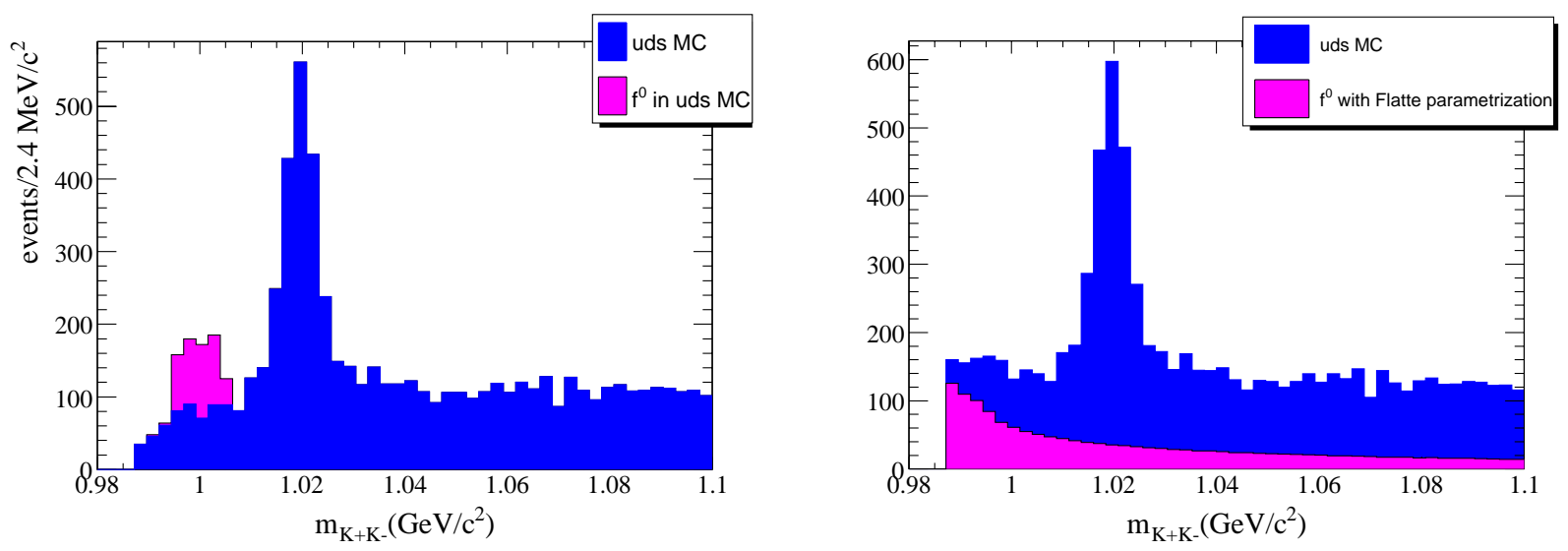

Figure 4.39: $K^{+} K^{-}$mass distribution from light quark simulation. The left plot is the generic $M C$. Apart from the $\phi$ peak one can see the $f_{0}$ contribution (magenta histogram). In the right plot, the $f_{0}$ has been replaced by a Flatte distribution.

After having corrected $c \bar{c}$ and uds MC, the data/MC comparison for the $K^{+} K^{-}$mass distribution is shown on Figure 4.40, normalizing the different MC samples to the corresponding luminosity. The distributions of the kinematic variables for the mass range from $1.1 \mathrm{GeV} / \mathrm{c}^{2}$ to $1.2 \mathrm{GeV} / \mathrm{c}^{2}$ are shown on Figure 4.41. In this mass region which is expected to be dominated by background contributions, data and simulation agree. 

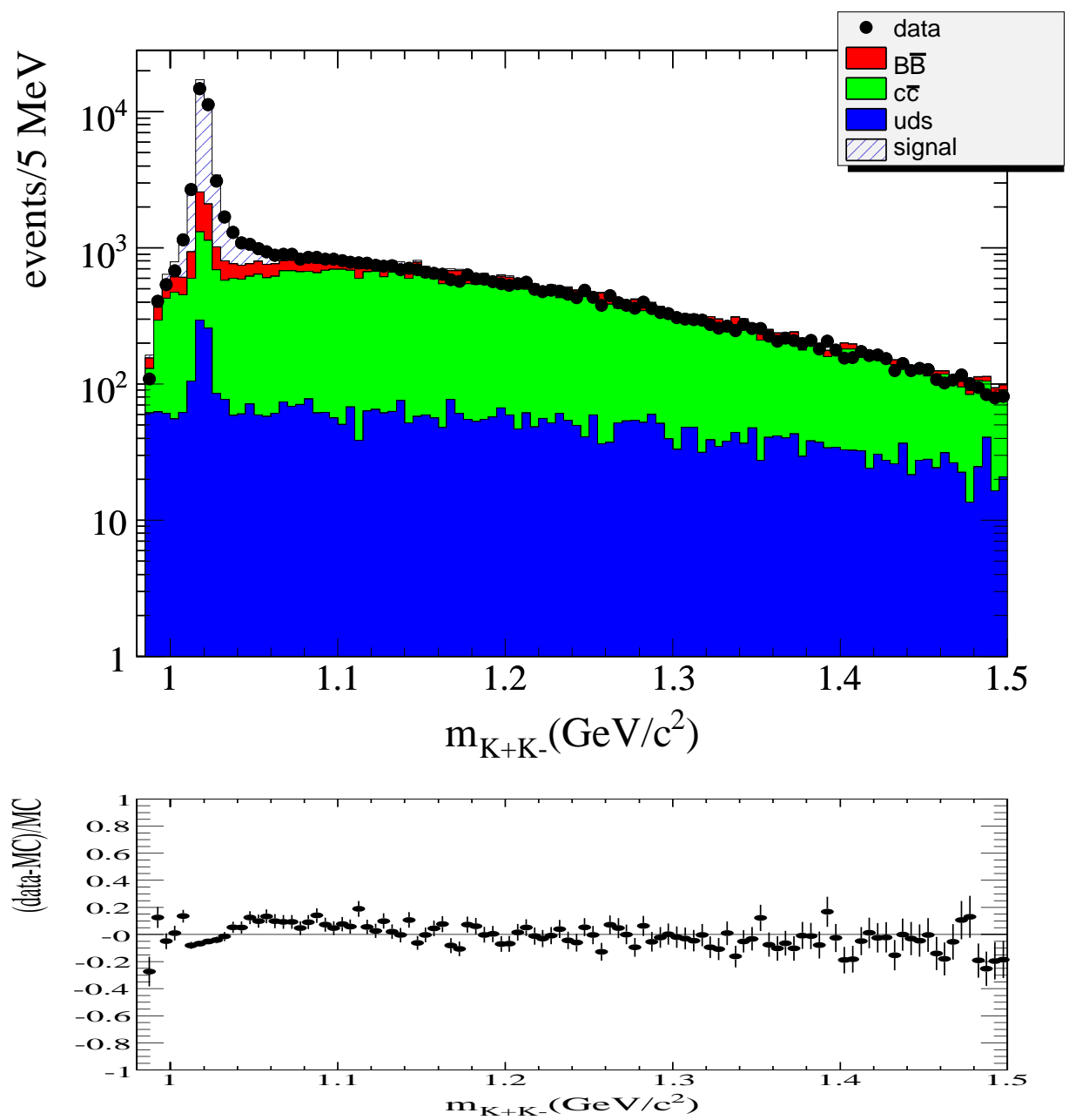

Figure 4.40: Data/MC comparison for $K^{+} K^{-}$mass distribution after having corrected $c \bar{c}$ and light quark simulation. 

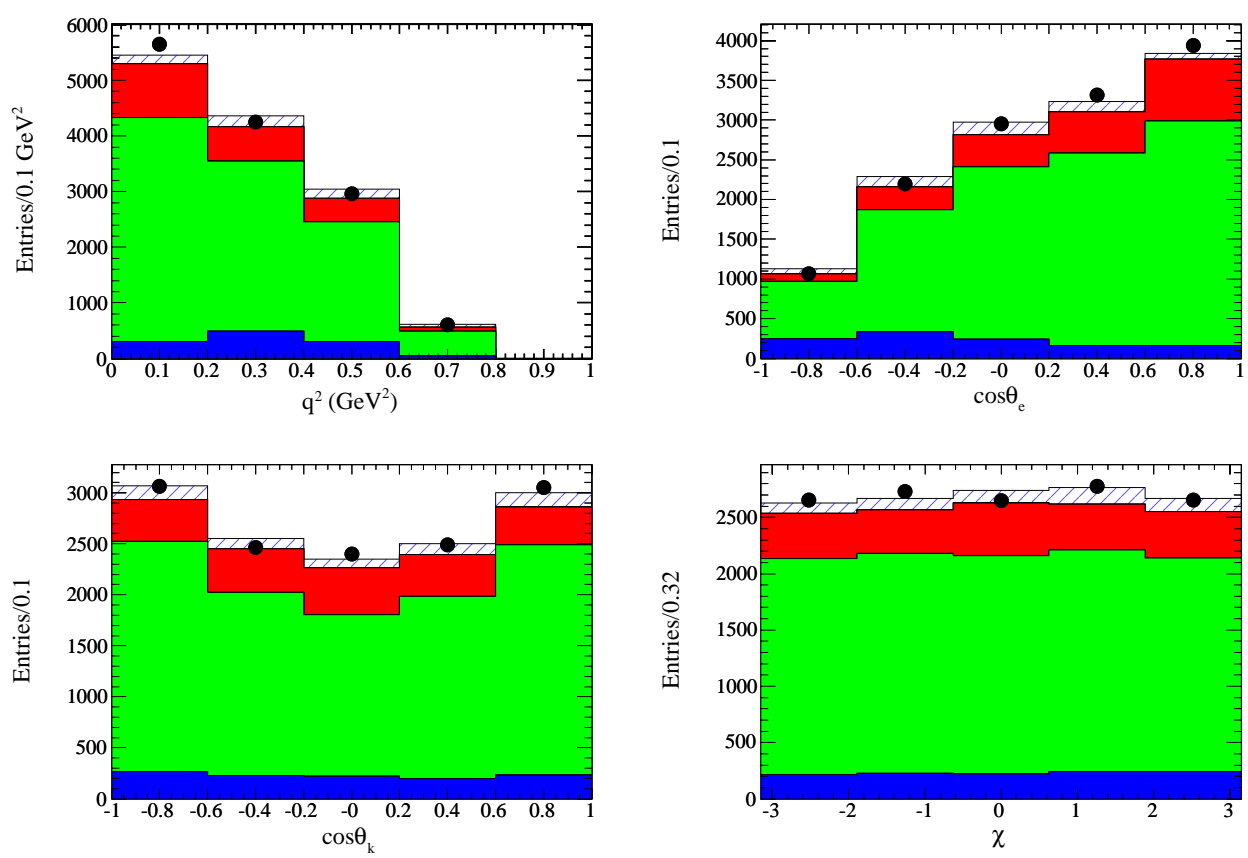

Figure 4.41: Kinematic variables for events between $1.1 \mathrm{GeV} / \mathrm{c}^{2}$ to $1.2 \mathrm{GeV} / \mathrm{c}^{2}$. The $M C$ is normalized to the number of events in data in order to compare the shape of the variables. 


\subsection{Summary of corrections applied to the Monte Carlo simulation}

The corrections applied on each type of simulated events are summarized in the following list:
signal:
Corrections as function of the $D_{s}$ momentum, spectator sys- tem momentum and $R_{2}$, smearing of the missing energy in the lepton hemisphere.
$c \bar{c}$ background: - events with a $D_{s}$ in the signal hemisphere: corrections as function of the $D_{s}$ momentum, $R_{2}$ and the multiplicity.
- events with a $D$ in the signal hemisphere: corrections as function of the $D$ momentum, $R_{2}$ and the multiplicity.
- events with a $\phi$ : correction on the rate of the $\phi$ production from charm hadron, no correction for $\phi$ from fragmentation.
- continuum $c \bar{c}$ background: for events with a kaon from frag- mentation and a kaon from a $D$ meson, correction as function of the $K$ momentum and direction relative to the $D$.

$B \bar{B}$ background: Corrections as function of $R_{2}$ and the multiplicity.

$u d s$ background: Correction of the $f_{0}$ shape, which implies a rescaling of 1.5 of uds continuum events.

\subsection{Corrected distributions}

Using simulated events normalized to the data luminosity, and including the corrections defined previously, we compare the projected distributions over the four kinematic variables for data and simulated events (Figure 4.42). On these plots, the simulated signal is obtained from the generic MC, in which the form factor model is ISGW2. One can see that this model does not agree well with the data.

In the same way, the distributions of the two Fisher discriminants can be seen on Figure 4.43, when all the cuts of the analysis are applied except the cut on the Fisher discriminants. Using the generic MC for the signal ${ }^{2}$, and before applying corrections, there is a large disagreement between the simulation and the data (upper plot). After having applied all corrections defined previously, the agreement is improved substantially (bottom plots). The remaining differences have been taken into account in the systematic uncertainties, performing the fit for several Fisher cut values.

\footnotetext{
${ }^{2}$ it has been verified that the shape of the Fisher discriminants does not depend on the form factors model
} 

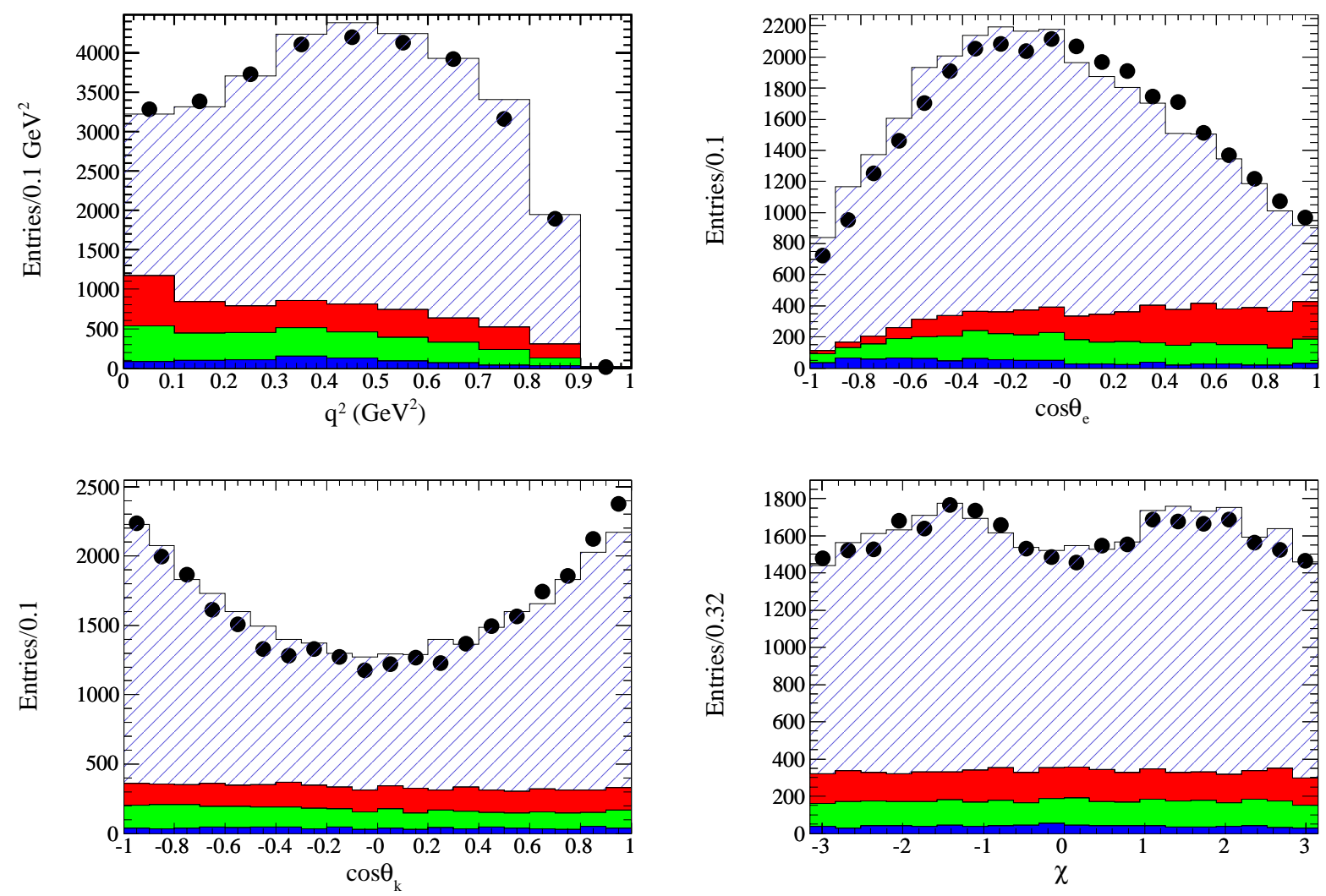

Figure 4.42: Projected distributions of the reconstructed values of the four kinematic variables which define the decay $D_{s}^{+} \rightarrow \phi e^{+} \nu_{e}$. Points with error bars correspond to data. Distributions given as histograms correspond to expectations from the $M C$, normalized to the same integrated luminosity. The variable $\cos \left(\theta_{V}\right)$ is measured using the $K^{+}$for a $D_{s}^{+}$candidate and the $K^{-}$for a $D_{s}^{-}$candidate. We can see that in data, this variable presents a positive asymmetry. 


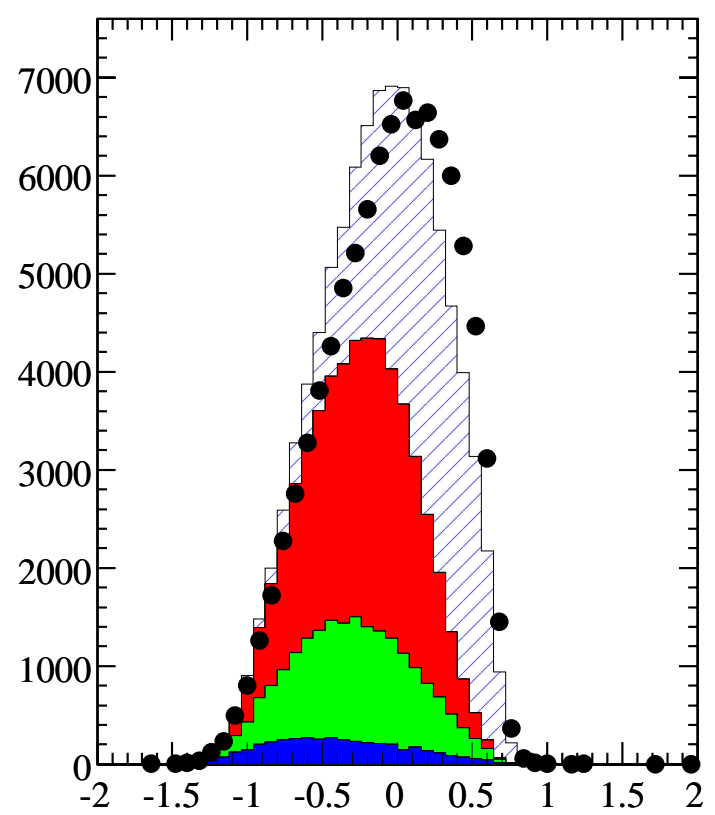

Fisher cc

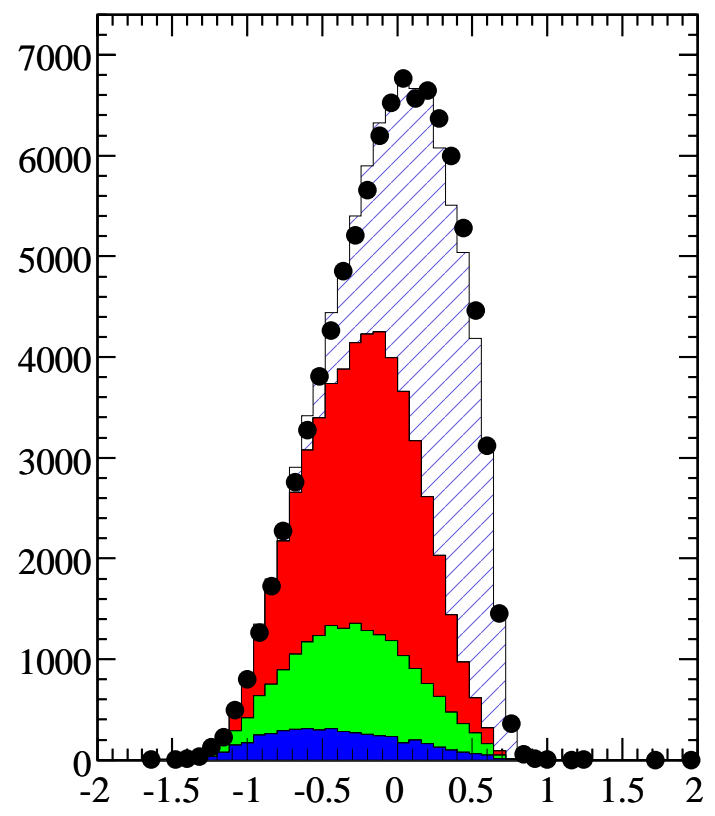

Fisher cc

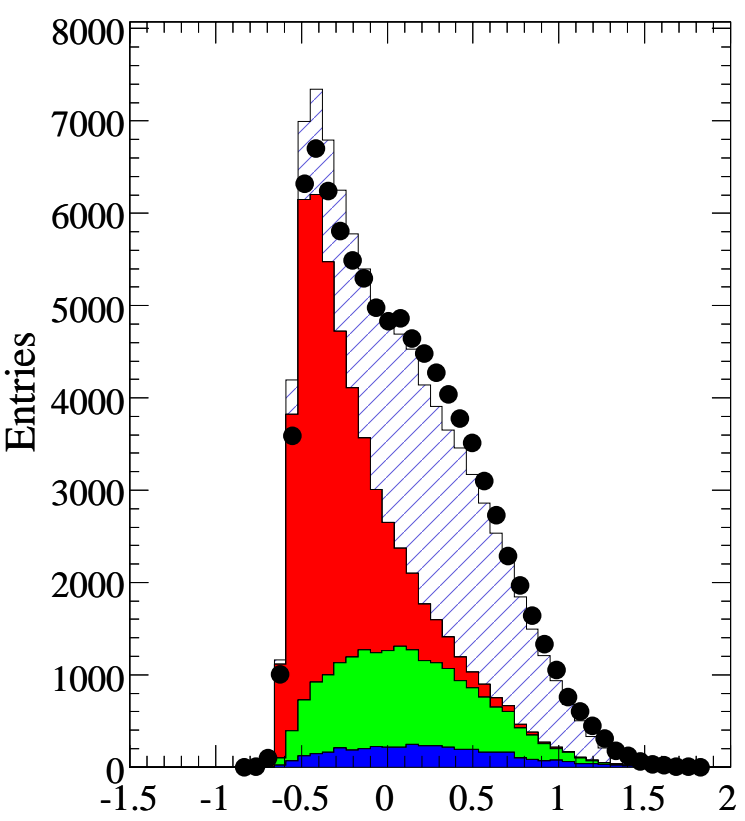

Fisher bb

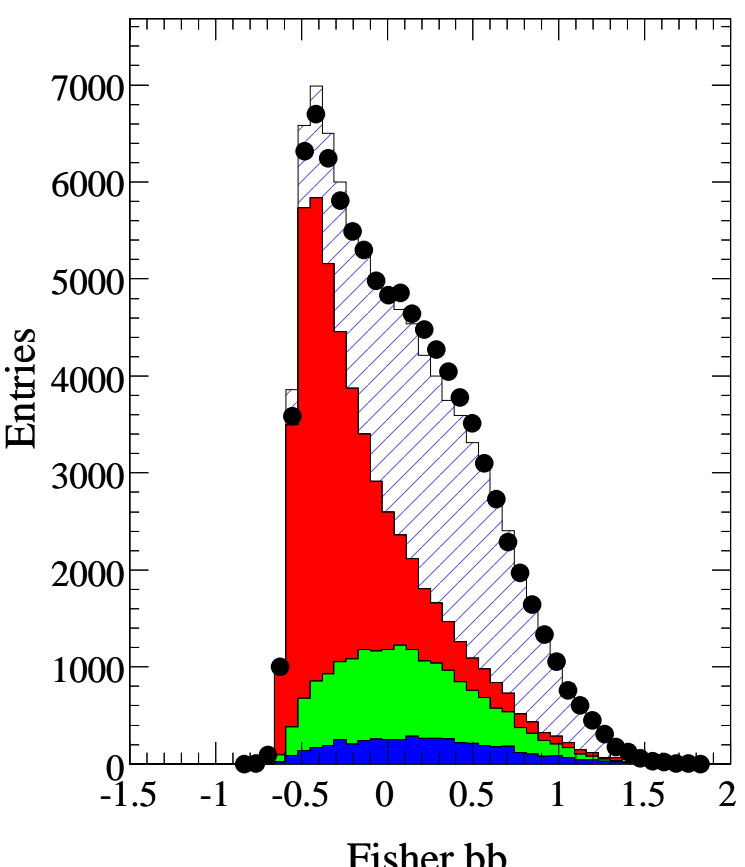

Fisher bb

Figure 4.43: Fisher discriminants distributions obtained with $D_{s}^{+} \rightarrow \phi e^{+} \nu_{e}$ events. Right plots are the Fisher discriminant against $B \bar{B}$ events and left plots correspond to the Fisher discriminant against $\bar{c}$ background events. Points with error bars correspond to real events. $M C$ is normalized to the data luminosity. The upper plots show the comparison of data with the signal from generic $M C$ when no corrections are done on the simulation. In the bottom plots, the different $M C$ components (signal and background) are corrected. 


\section{Chapter 5}

\section{Determination of form factors parameters}

'Would you tell me', said Alice, a little timidly 'why you are painting those roses?'

Five and Seven said nothing, but looked at Two. Two began in a low voice, 'Why the fact is, you see, Miss, this here ought to have been a red rose-tree, and we put a white one by mistake; and if the Queen was to find out, we should all have our heads cut off, you know.'

In this chapter we describe the fit procedure used to extract the form factors parameters and its validation through different tests.

\subsection{Fit procedure}

Events are distributed in 4-dimensional bins in the variables $q_{r}^{2}, \cos \left(\theta_{V}\right)_{r}, \cos \left(\theta_{e}\right)_{r}$ and $\chi_{r}$, where the index $r$ stands for the reconstructed value of the variables. The values of the fitted parameters are obtained by minimizing a negative log-likelihood distribution:

$$
\mathcal{L}=-\sum_{i=1}^{n \text { bins }} \ln \mathcal{P}\left(n_{i}^{\text {data }} \mid n_{i}^{M C}\right)
$$


In this expression, $\mathcal{P}\left(n_{i}^{\text {data }} \mid n_{i}^{M C}\right)$ is the Poisson probability to observe $n_{i}^{\text {data }}$ data events, with $n_{i}^{M C}$ expected in bin $i$.

Considering the experimental resolutions (Figure 3.9), five bins have been used in each dimension which corresponds to 625 bins in total.

The expected number of events in bin $i$ results from:

- $\phi e^{+} \nu_{e}$ signal events;

- the combinatorial background under the $\phi$;

- the background coming from $\phi$ mesons produced in the fragmentation process or in $B$ or $D$ decays.

The number of signal events expected in bin $i$ is obtained in the following way. A dedicated sample of signal events is generated with the SP8 release. The form factor model used to produce this sample is the simple pole model with values of the parameters $\left(\lambda_{k}^{0}\right): r_{V}=$ $1.5, r_{2}=0.7$ and the pole masses are fixed to the spectroscopic values $\left(m_{A}=2.5 \mathrm{GeV} / \mathrm{c}^{2}\right.$, $\left.m_{V}=2.1 \mathrm{GeV} / \mathrm{c}^{2}\right)$. PHOTOS is used to simulate radiative effects. This sample is analyzed in the same way as the data, using the same reconstruction procedure, and it is corrected with the weights defined in Section 4.1.1. Each event $j$ is weighted using the differential decay rate given in Equation 1.25. The weight $w_{j}\left(\lambda_{k}\right)$ can be written as:

$$
w_{j}\left(\lambda_{k}\right)=\frac{q^{2} \mathcal{I}_{R}\left(\lambda_{k}\right)+\mathcal{I}_{R}^{S}\left(\lambda_{k}\right)+\mathcal{I}_{R}^{S P}\left(\lambda_{k}\right)}{q^{2} \mathcal{I}_{R}\left(\lambda_{k}^{0}\right)},
$$

where $\mathcal{I}_{R}$ is defined by Equation 1.26 and the expressions used for $\mathcal{I}_{R}^{S}$ and $\mathcal{I}_{R}^{S P}$ are explained in Section 1.2.4. The numerator contains the fitted values $\lambda_{k}$ of the parameters whereas the denominator corresponds to the distribution used in the generation of the signal MC, with parameters $\lambda_{k}^{0}$. In the following, the parameters $\lambda_{k}$ are $r_{V}, r_{2}$, quantities which define the shape of the form factors $\left(m_{A}\right.$ and $\left.m_{V}\right)$ and the parameter related to $S$-wave amplitude, $r_{0}$.

The expected average number of background events in each bin is obtained after having noted that the background rate is flat versus two of the four dimensions: $\cos \left(\theta_{V}\right)$ and $\chi$, when the asymmetry is not taken into account. We thus use a constant average value for these two variables:

$$
n_{i_{q^{2}}, i_{\cos \left(\theta_{e}\right)}, i_{\cos \left(\theta_{V}\right), i_{\chi}}^{b c k g .}}=\frac{\sum_{j, k=1}^{n b i n_{\cos \left(\theta_{V}\right)}, n b i n_{\chi}} n_{i^{2}, i_{\cos \left(\theta_{e}\right), j, k}}^{b c k g .}}{n b i n_{\cos \left(\theta_{V}\right)} n b i n_{\chi}}
$$


This expression applies to each component of background events. These components are then normalized to correspond to the integrated luminosity used for real data events.

The absolute normalization for signal events $\left(N_{S}\right)$ is left free to vary in the fit. In each bin $(i)$, the expected number of events is then evaluated to be:

$$
n_{i}^{M C}=N_{S} \frac{\sum_{j=1}^{n_{i}^{\text {signal }}} w_{j}\left(\lambda_{k}\right)}{W_{\text {tot }}\left(\lambda_{k}\right)}+n_{i}^{\text {bckg. }}
$$

$W_{t o t}\left(\lambda_{k}\right)=\sum_{j=1}^{N^{s i g n a l}} w_{j}\left(\lambda_{k}\right)$ is the sum of the weights for all simulated signal events. $N_{S}$ and $\lambda_{k}$ are the fit parameters. To avoid having to introduce finite ranges for the pole masses, we define $m_{i}=1+\lambda_{i}^{2}$. This expression ensures that $m_{i}^{2}$ is always larger than $q_{\text {max. }}^{2} \simeq 0.9 \mathrm{GeV}^{2}$, thus avoiding a singularity in the simple pole parametrization of the form factors.

\section{$5.2 \quad$ Fit quality}

The fit quality is evaluated in the following way: in each bin, the measured number of events is compared with expectations from the background and fitted signal components. We can define the quantity $x_{i}$ :

$$
x_{i}=\frac{n_{i}^{\text {data }}-n_{i}^{\text {estimated }}}{\sqrt{n_{i}^{\text {estimated }}+\sigma_{i}^{2}(\text { bkg })+\sigma_{i}^{2}(\text { signal })}}, i=1, \text { nbins }
$$

In this expression, we take into account the uncertainties in the evaluation of the average background level and the uncertainties from the weighting procedure $\sigma_{i}^{2}(b k g)=n_{i}^{b c k g} \cdot / 25$ and $\sigma_{i}^{2}$ (signal) $=N_{S}^{2} \sum_{j=1}^{n_{i}^{\text {signal }}} w_{j}^{2} / W_{\text {tot }}^{2}$. Then we define $\chi^{2} \equiv \sum_{i=1}^{n b i n s} x_{i}^{2}$. The distribution of the number of data events per bin can be seen in Figure 5.1. As this number can be small, conditions for Gaussian statistics are not fulfilled and a more appropriate test consists in comparing values of the minimum for the negative log-likelihood, obtained with toy experiments and in real data.

\subsection{Parameters sensitivity}

To evaluate the sensitivity of the analysis on parameter's values, we have done a study using Monte Carlo generated events. A sample has been generated using values of the parameters similar to experimental measurements : $r_{V}=1.5, r_{2}=0.7, m_{A}=2.5 \mathrm{GeV} / \mathrm{c}^{2}$ and $m_{V}=2.1 \mathrm{GeV} / \mathrm{c}^{2}$. We have then reweighted this sample varying separately $r_{V}$ and 


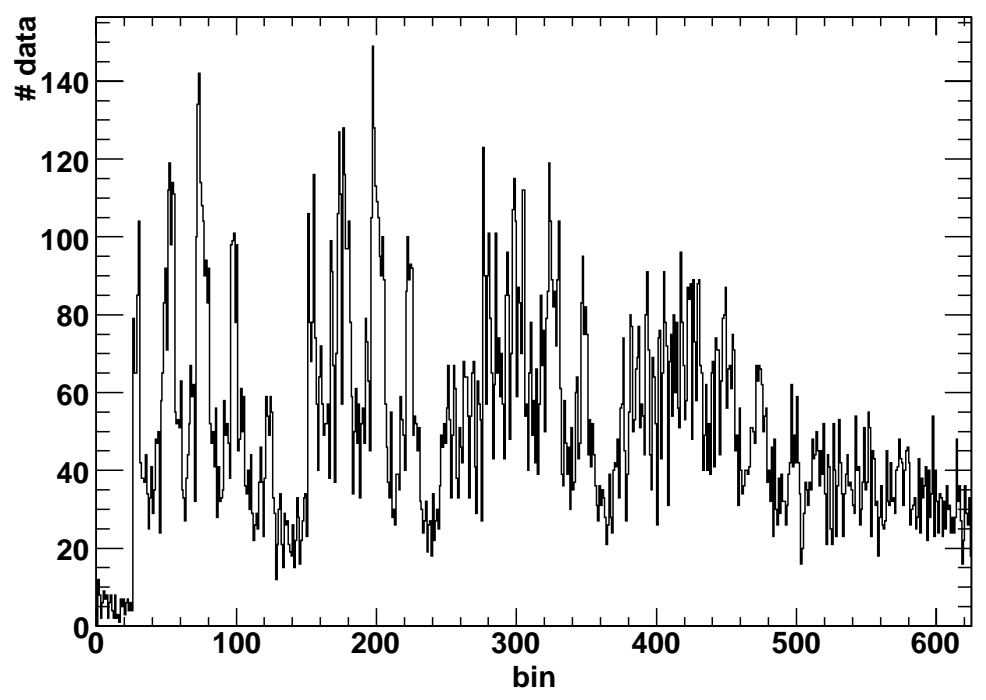

Figure 5.1: number of data events in each of the 625 bins.

$r_{2}$ by $3 \sigma, \sigma$ corresponds to the statistical error of the preliminary version of this analysis, performed with $78.5 \mathrm{fb}^{-1}\left(\sigma_{r_{V}}=0.065\right.$ and $\left.\sigma_{r_{2}}=0.053\right)$. Distributions of the four variables and their corresponding ratios can be seen on Figure 5.2 and 5.3.

We have also varied $m_{A}$ and $m_{V}$ by $0.5 \mathrm{GeV} / \mathrm{c}^{2}$. The results can be seen on Figure 5.4 and 5.5 and show that the kinematic variables are sensitive to $m_{A}$ but the sensitivity on $m_{V}$ is very weak.

We can see that the determination of $m_{A}$ depends on $q^{2}$ and $\cos \left(\theta_{e}\right)$ distributions, whereas the determination of $r_{2}$ depends on these two variables and also on $\cos \left(\theta_{V}\right)$, which explains why the data are sensitive to $m_{A}$ and $r_{2}$. On the other hand, the sensitivity to $m_{V}$ in mainly in the $\cos \left(\theta_{e}\right)$ distribution, as for $r_{V}$, which explains the fact that these two variables are correlated, and cannot both be treated as free parameters in the fit. To assess the sensitivity of the present measurements on $m_{A}$ and $m_{V}$, we have also done a scan of the negative log likelihood distribution near its minimum (Figure 6.4). 

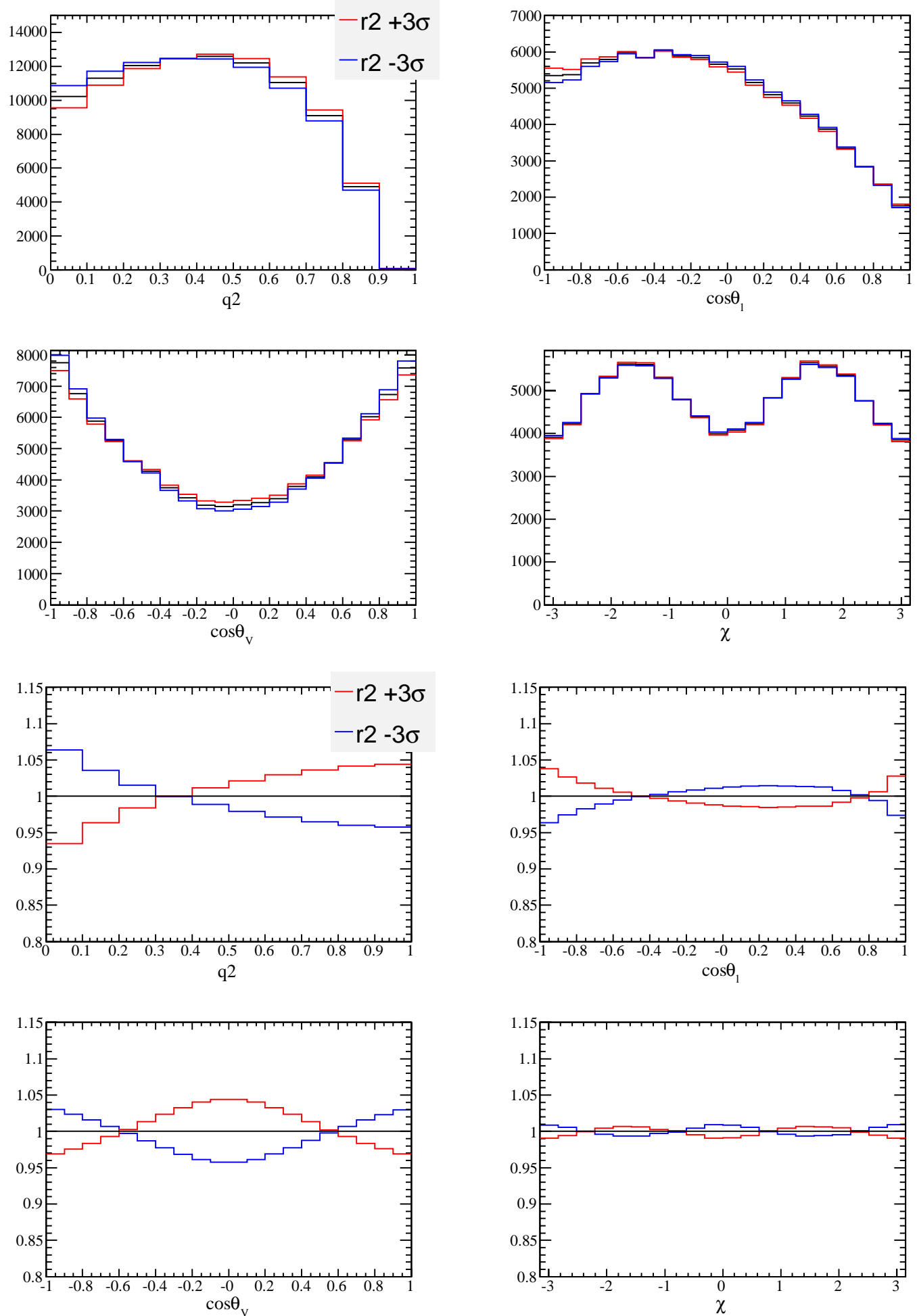

Figure 5.2: Distribution of the kinematic variables for different values of $r_{2}$. The reference histogram $\left(r_{2}^{r e f}=0.7\right)$ is in black. The bottom plots correspond to the ratio between the histograms $r_{2}^{r e f} \pm 3 \sigma$ and the reference histogram. 

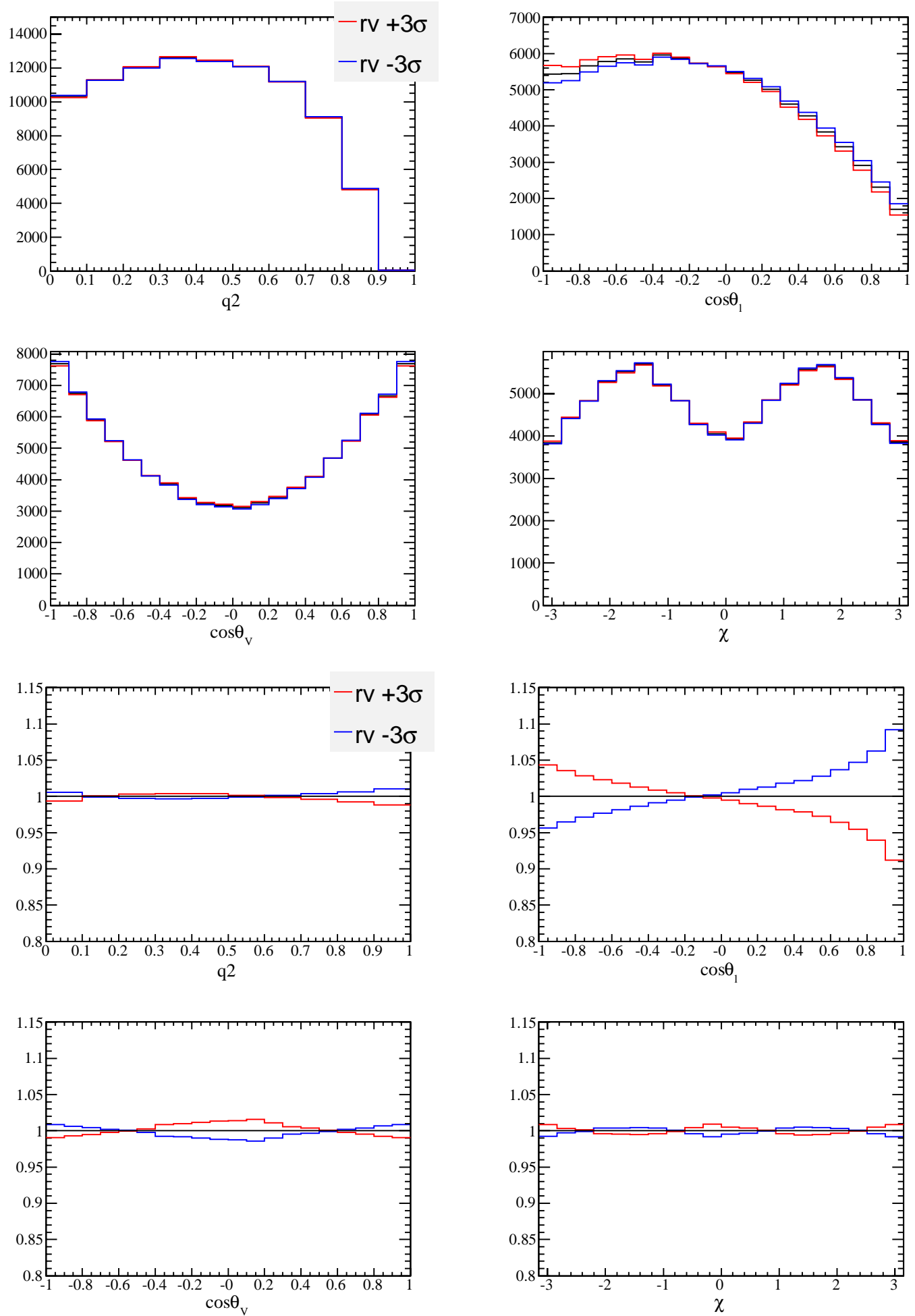

Figure 5.3: Distribution of the kinematic variables for different values of $r_{V}$. The reference histogram $\left(r_{V}^{r e f}=1.5\right)$ is in black. The bottom plots correspond to the ratio between the histograms $r_{V}^{r e f} \pm 3 \sigma$ and the reference histogram. 

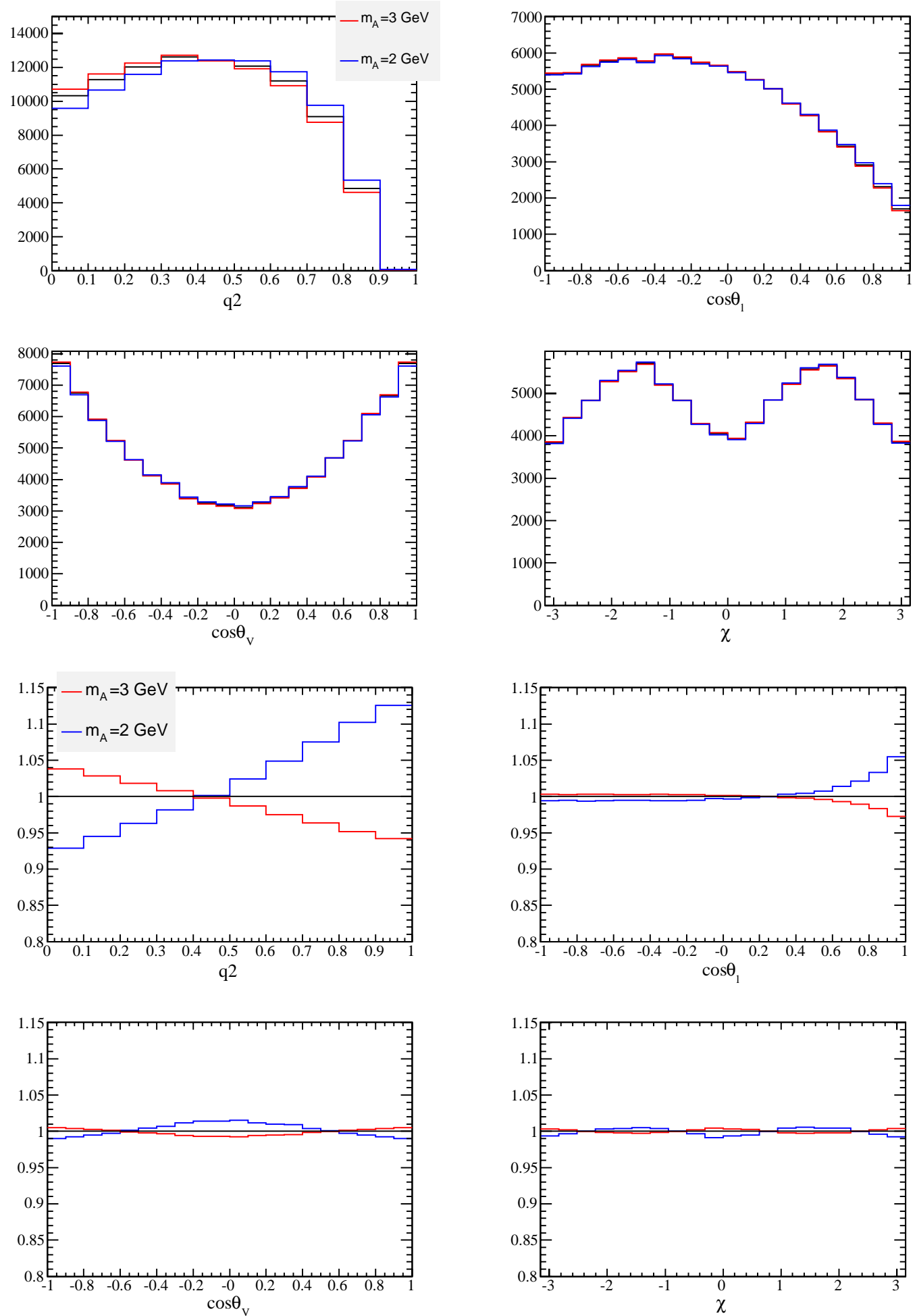

Figure 5.4: Distribution of the kinematic variables for different values of $m_{A}$. The reference histogram $\left(m_{A}=2.5 \mathrm{GeV} / \mathrm{c}^{2}\right)$ is in black. The bottom plots correspond to the ratio between the histogram $m_{A}^{r e f} \pm 0.5 \mathrm{GeV} / \mathrm{c}^{2}$ and the reference histogram. 

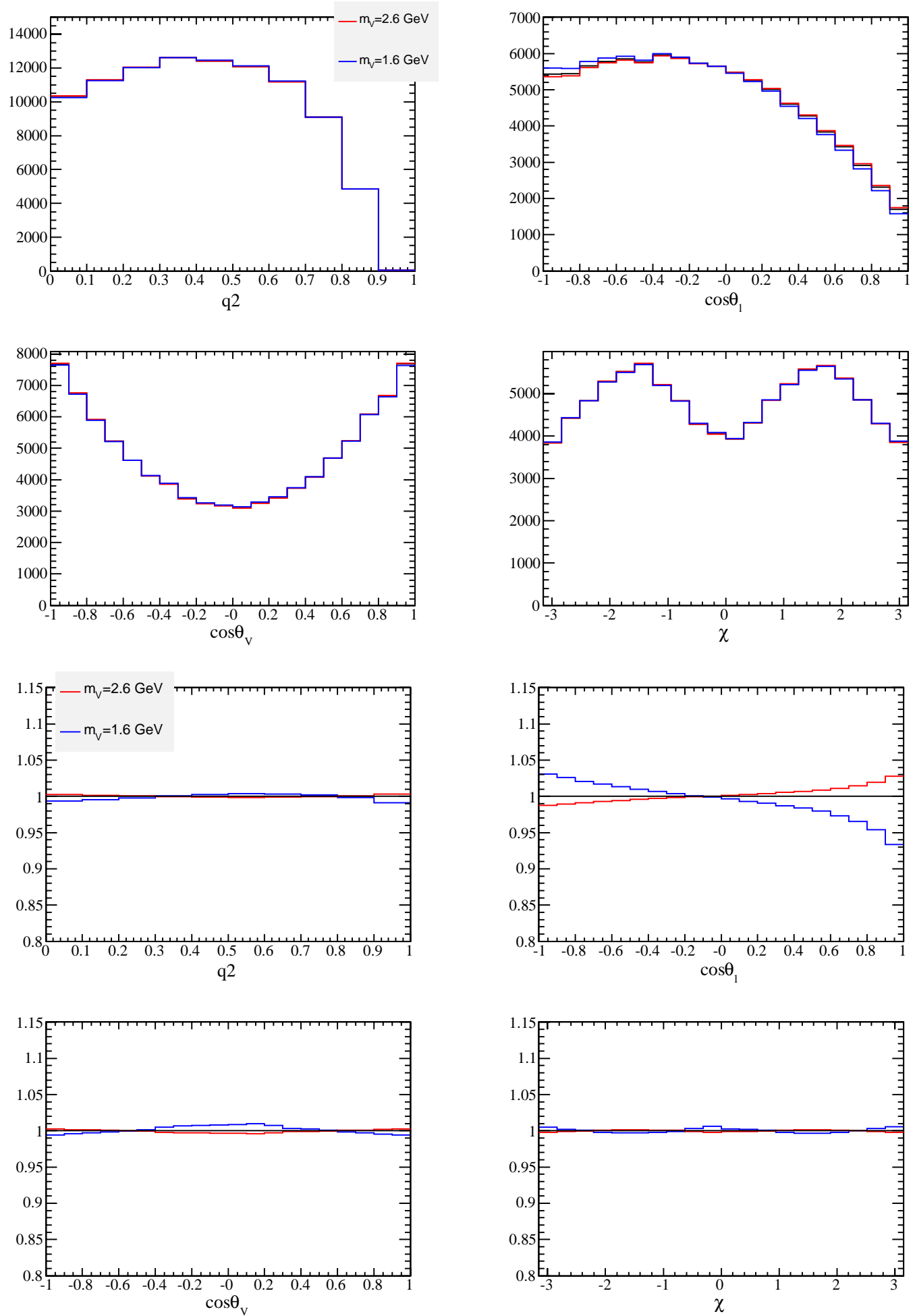

Figure 5.5: Distribution of the kinematic variables for different values of $m_{V}$. The reference histogram $\left(m_{V}=2.1 \mathrm{GeV} / \mathrm{c}^{2}\right)$ is in black. The bottom plots correspond to the ratio between the histogram $m_{V}^{r e f} \pm 0.5 \mathrm{GeV} / \mathrm{c}^{2}$ and the reference histogram. 


\subsection{Results on simulated events}

Two tests have been performed to check the fitting procedure.

\subsubsection{Toy simulations}

In the adopted fitting procedure, two sources of statistical fluctuations are not included. As a result, statistical uncertainties obtained from a fit on data or on the simulation can be underestimated and the values of the fitted parameters can have biases.

These sources of additional statistical fluctuations are coming from the finite statistics of simulated signal events and from uncertainties in the estimate of the average number of background events in each bin.

These statistical effects, on the fitted values of the parameters $r_{V}$ and $r_{2}$ have been evaluated using toy simulations. Experiments have been generated with a sample of about 25000 signal events, with the following parameters values: $r_{V}=1.6, r_{2}=0.8, m_{A}=$ $2.5 \mathrm{GeV} / \mathrm{c}^{2}$ and $m_{V}=2.1 \mathrm{GeV} / \mathrm{c}^{2} .7000$ background events have been added to each experiment. Background events are generated with a flat distribution in the kinematic variables. For each fake experiment we generate also a sample of events which are used as simulated data in the fit. 225000 signal events are generated with a slightly different model for the form factors. In this second sample we have used the values: $r_{V}=1.5, r_{2}=0.7$, $m_{A}=2.5 \mathrm{GeV} / \mathrm{c}^{2}$ and $m_{V}=2.1 \mathrm{GeV} / \mathrm{c}^{2}$. For each fit, not only the "fake experiment" is generated but also the sample of pure signal and the background distributions are created. Results obtained using 1000 such experiments are given in Figure 5.6.

The sigma of the pull distributions is 1.115 for $r_{2}$ and 1.055 for $r_{V}$, biases are -0.008 and -0.044 respectively. Uncertainties on these numbers are \pm 0.03 . The exercise has been repeated without including background events. The biases are then -0.020 and -0.013 for $r_{V}$ and $r_{2}$, respectively. The sigma values of these distributions are 1.063 and 1.085 .

The systematic uncertainty attached to the statistics of simulated events and to the statistical uncertainties on the average number of background events in each bin, has been evaluated, from the increase of the sigma of the pull distribution, relative to unity.

$$
\sqrt{1.1^{2}-1} \times \sigma_{f i t .} \simeq 0.46 \times \sigma_{f i t}
$$

where 0.1 has been taken as representative value for the increase of the $\sigma$ of the pull, relative to unity. 

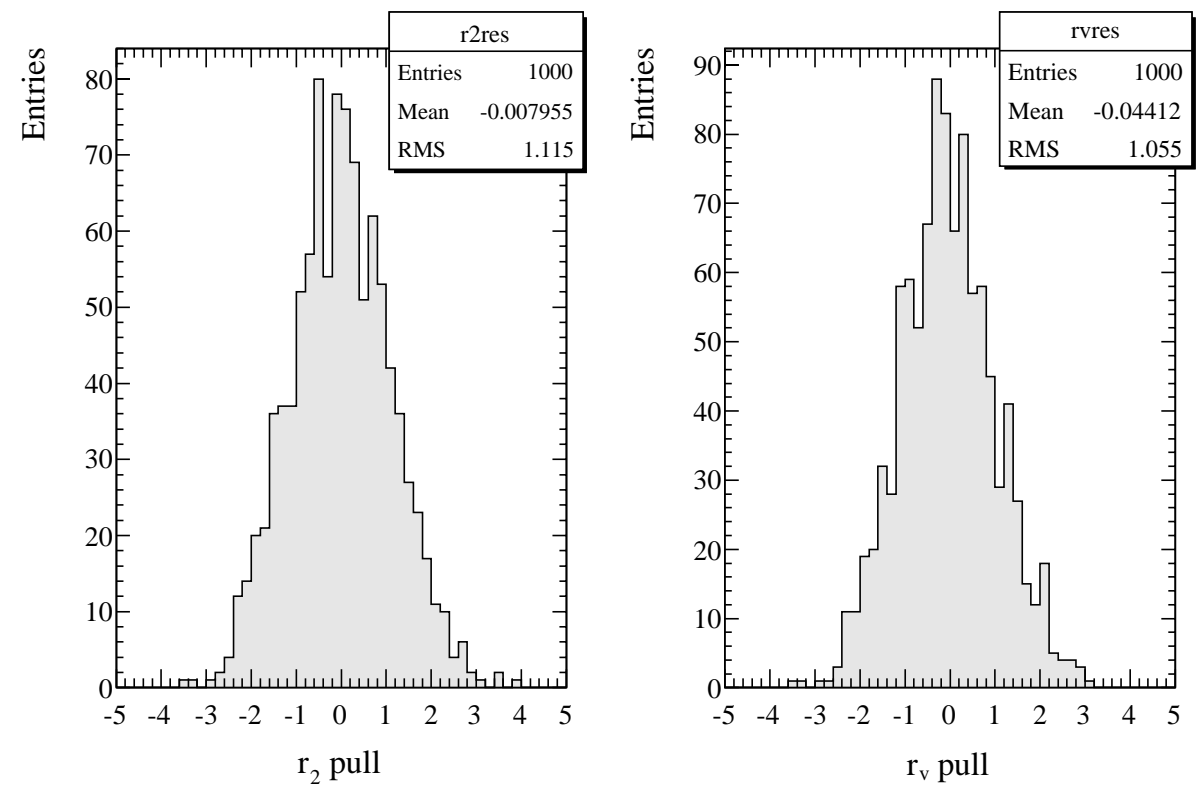

Figure 5.6: Pull distributions for the fitted values of $r_{V}$ and $r_{2}$ obtained using a fraction of background $(B / S)$ of $28 \%$.

The same study has been repeated fitting also the axial-vector pole mass as it can be seen on Figure 5.7. For $r_{2}$ and $r_{V}$, the biases are less than 0.05, and the sigma values are 1.074 and 1.033. For these parameters we use the same systematic uncertainty as the one defined previously. For the parameter $\lambda_{A}$, the pull distribution is not symmetric and we see a bias of -0.2. Nevertheless, the distribution of the difference between the fitted value of $\lambda_{A}$ and its generated value $\lambda_{A}^{0}$, shown in Figure 5.8, does not indicate a bias.

In fact, the variable $\lambda_{A}$ has two properties which can lead to a non Gaussian pull distribution:

- it has asymmetric errors;

- the errors depends on its value, as shown in Figure 5.9.

These characteristics are related to the pole dominance: when the pole mass is closer to the physical region of $q^{2}$, the fit is more sensitive and errors are smaller. This explains why negative errors are smaller than positive ones (this is visible also on the scan of the maximum likelihood value as function of $m_{A}$, in Figure 6.4). Thus, the fit is underestimating the errors of $\lambda_{A}$ if the fitted value is smaller than the true one, and it overestimates the errors if the fitted value is greater than the real one. This is an intrinsic property of this fit and we have not found a way to correct it. 

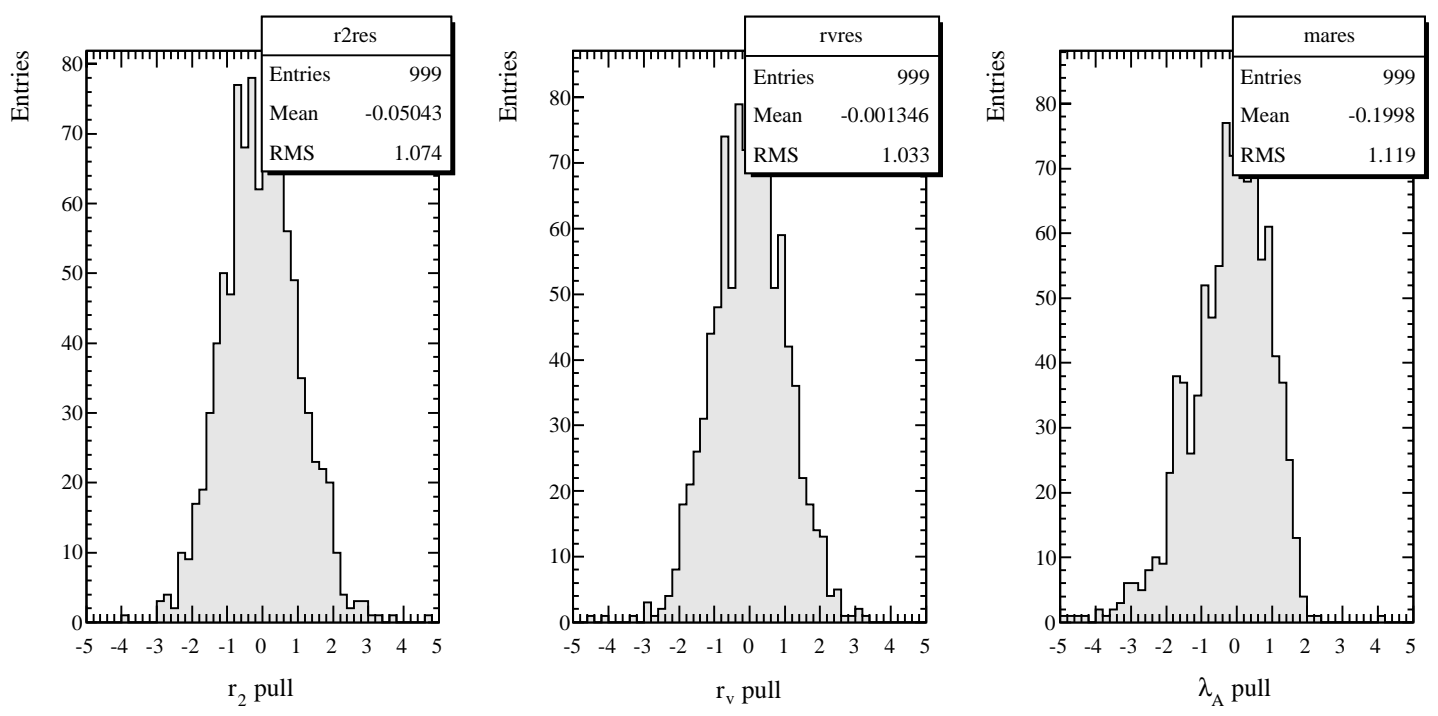

Figure 5.7: Pull distributions for the fitted values of $r_{V}, r_{2}$ and $\lambda_{A}$ obtained using a fraction of background $(B / S)$ of $28 \%$.

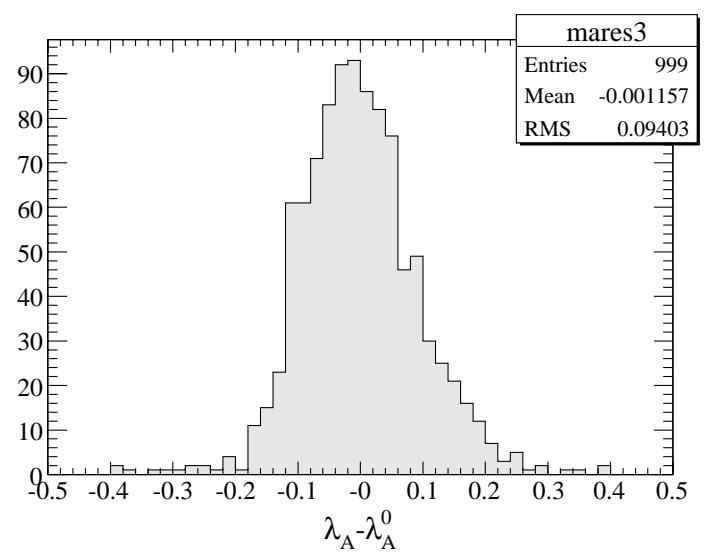

Figure 5.8: Difference between the fitted value of $\lambda_{A}$ and the true value $\lambda_{A}^{0}$. 
For the other fitted parameters, this effect is not present. Errors of the parameters $r_{2}$ and $r_{V}$ are shown in Figure 5.10 as function of the parameters value. The errors differ from the mean error by less than $5 \%$ whereas this difference can vary between $-20 \%$ and $+50 \%$ for the parameter $\lambda_{A}$.

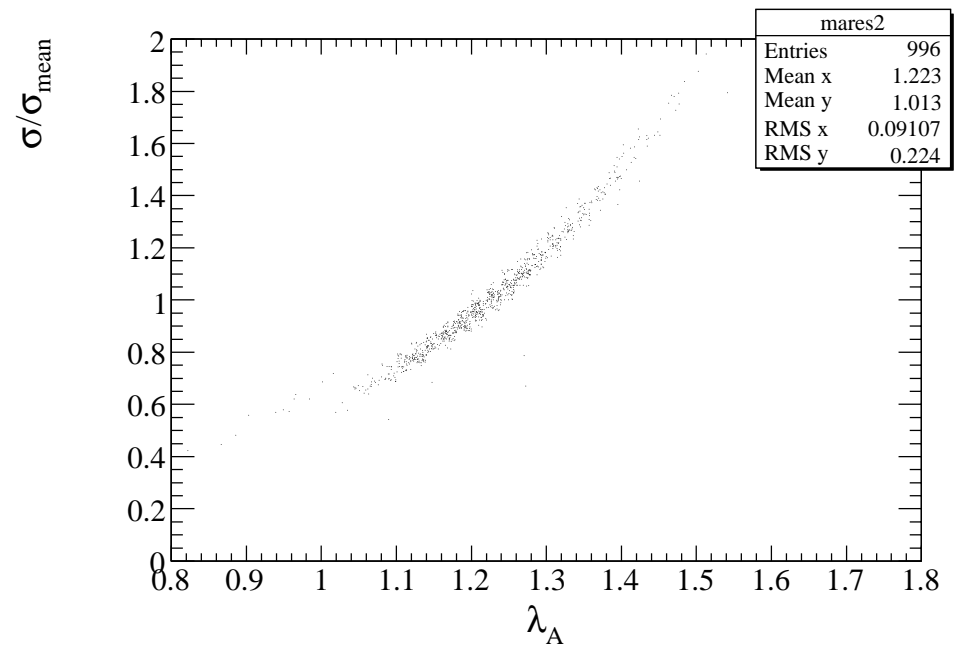

Figure 5.9: Error on $\lambda_{A}$ divided by the mean error value as function of $\lambda_{A}$, as obtained with toys.

\subsubsection{Analysis of fully simulated events}

In the second test, we use a sample of Monte Carlo signal events generated with the simple pole model and using PHOTOS for radiative corrections. These events are analyzed through the same algorithm as real data events, and no background is added. 68674 events remain after this procedure, they are fitted using an independent Monte Carlo sample of 5 times the "fake data" statistics (342655 events) and results are given in Table 5.1. $r_{V}$ and $r_{2}$ differ by about $1 \sigma$ from the expected values.

\begin{tabular}{|c|c|c|}
\hline parameter & Exact value & Fitted value \\
& & \\
\hline$N_{S}$ & 68674 & $68640 \pm 262$ \\
$r_{V}$ & 1.5 & $1.519 \pm 0.023$ \\
$r_{2}$ & 0.7 & $0.667 \pm 0.021$ \\
\hline
\end{tabular}

Table 5.1: Comparison between exact and fitted values on fully simulated events.

This sample has also been used to test the simultaneous fit of the form factor ratio $r_{V}$ and $r_{2}$ and of the pole masses. With the present analysis one can determine $m_{A}$ but the 

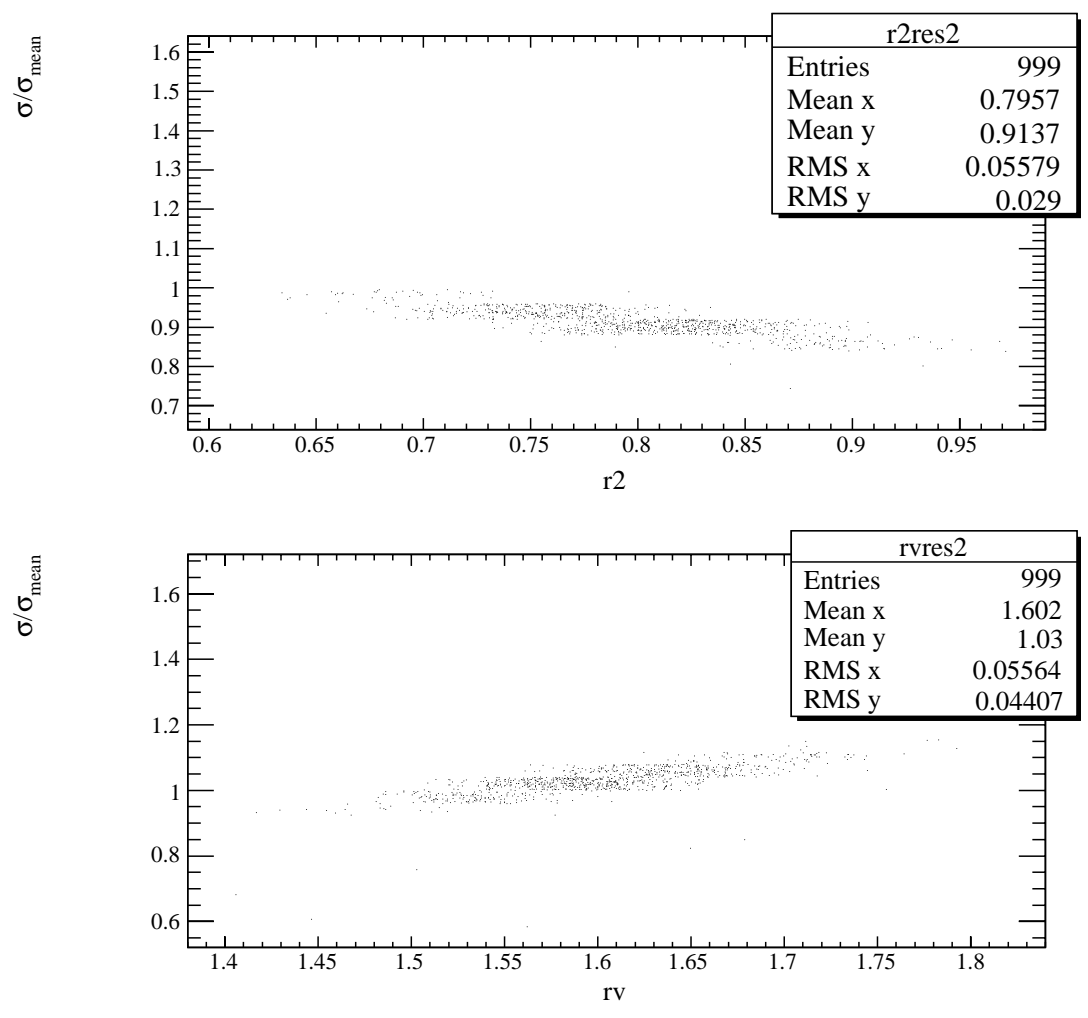

Figure 5.10: Error on $r_{2}$ (top) and $r_{V}$ (bottom) divided by the mean error value as function of the corresponding parameter value, as obtained with toys. 
sensitivity on $m_{V}$ is weak and we have fixed its value to $2.1 \mathrm{GeV} / \mathrm{c}^{2}$. Results are given in Table 5.2, and differ by less than $1 \sigma$ from the expected values. For these two fits, the $\chi^{2}$

\begin{tabular}{|c|c|c|}
\hline parameter & Exact value & Fitted value \\
\hline$N_{S}$ & 68674 & $68640 \pm 262$ \\
$r_{V}$ & 1.5 & $1.516 \pm 0.027$ \\
$r_{2}$ & 0.7 & $0.675 \pm 0.039$ \\
$m_{A}$ & $2.5 \mathrm{GeV} / \mathrm{c}^{2}$ & $\left(2.54_{-0.15}^{+0.18}\right) \mathrm{GeV} / \mathrm{c}^{2}$ \\
\hline
\end{tabular}

Table 5.2: Comparison between exact and fitted values on fully simulated events.

probability for nbins -3 or 4 degrees of freedom is equal to $89 \%$. 


\section{Chapter 6}

\section{Results}

'Would you tell me, please, which way I ought to go from here?'

'That's depends a good deal on where you want to get to,' said the Cat.

'I don't much care where -', said Alice.

'Then it doesn't matter which way you go', said the Cat.

'- so long as I get somewhere,' Alice added as an explanation.

'Oh, you're sure to do that,' said the Cat, 'if you only walk long enough.'

To compare with previous analyzes, data are first fitted not considering a possible Swave component. Then this component is included in the fit and a signal with more than $5 \sigma$ significance is observed through its interference with the $\phi$ meson.

\subsection{Fit values}

Normalizing background contributions to the analyzed luminosity and using simulated signal events generated according to a simple pole mass model, a fit to data events has been performed. The Monte Carlo sample is equal to 7 times the data statistics. In this fit, all corrections defined in chapter 4 are applied. Using fixed values for the pole masses the following values have been obtained:

$$
N_{S}=25157 \pm 177, r_{V}=1.799 \pm 0.046 r_{2}=0.813 \pm 0.036
$$


With the correlation matrix:

$$
\left(\begin{array}{ccc}
1 & 0.059 & 0.001 \\
0.059 & 1 & 0.138 \\
0.001 & 0.138 & 1
\end{array}\right)
$$

The distribution of the $x_{i}$ values (see equation 5.5) obtained for the 625 bins is given in Figure 6.1-left. A Gaussian fit of this distribution has a sigma close to 1.
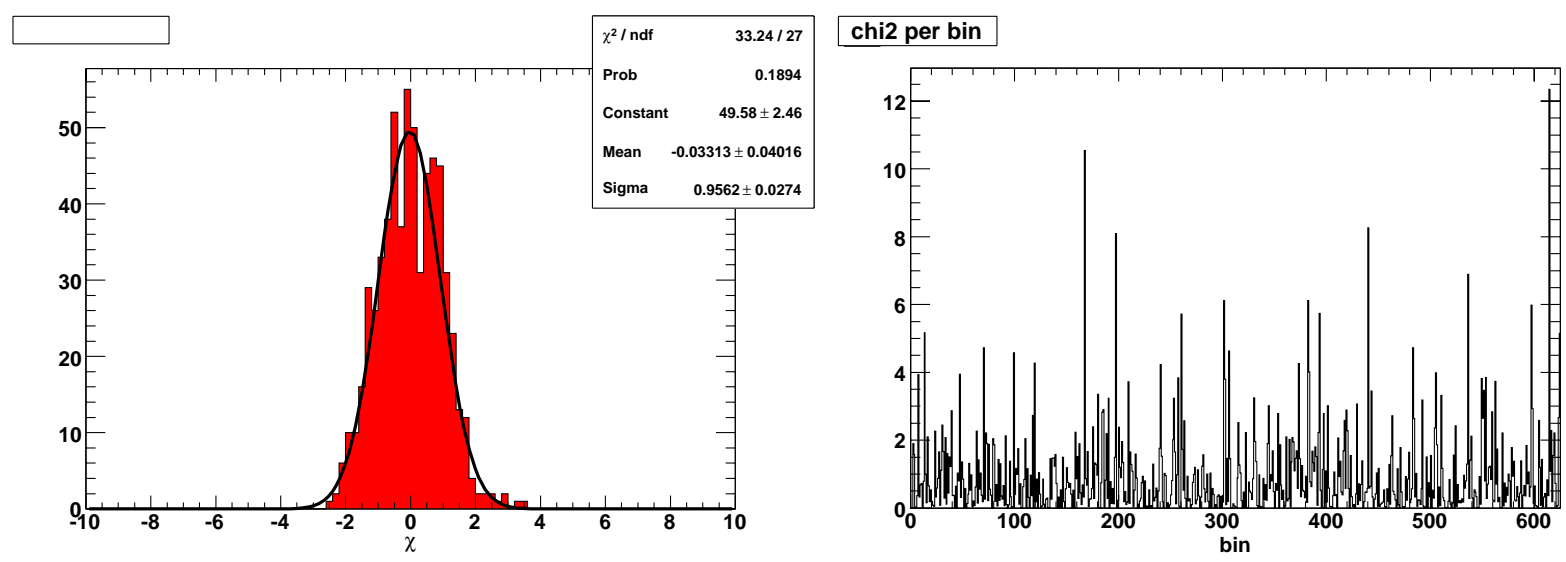

Figure 6.1: Left: Distribution of the values of the variable $x_{i}$ in each bin. Right: Values of $x_{i}^{2}$ versus the bin number.

The corresponding $\chi^{2}$ probability for nbins -3 degrees of freedom is equal to $80 \%$. Fitted distributions, projected over the four variables, are displayed in Figure 6.2.

Values corresponding to the minimum of the negative log-likelihood for the 1000 toy experiments have been displayed on Figure 6.3. The value measured with real data events is 2082 which seems reasonable.

If we fix only the pole mass of the vector form factor $\left(m_{V}\right)$, the following values and correlation matrix have been obtained:

$$
\begin{gathered}
\lambda_{A}=\left(1.14_{-0.08}^{+0.10}\right) \mathrm{GeV}^{1 / 2} / \mathrm{c}, \mathrm{N}_{\mathrm{S}}=25146 \pm 177, \mathrm{r}_{\mathrm{V}}=1.831 \pm 0.060, \mathrm{r}_{2}=0.758 \pm 0.071 . \\
\qquad\left(\begin{array}{cccc}
1 & -0.070 & 0.612 & -0.859 \\
-0.070 & 1 & -0.089 & 0.060 \\
0.612 & -0.089 & 1 & -0.581 \\
-0.859 & 0.060 & -0.581 & 1
\end{array}\right)
\end{gathered}
$$



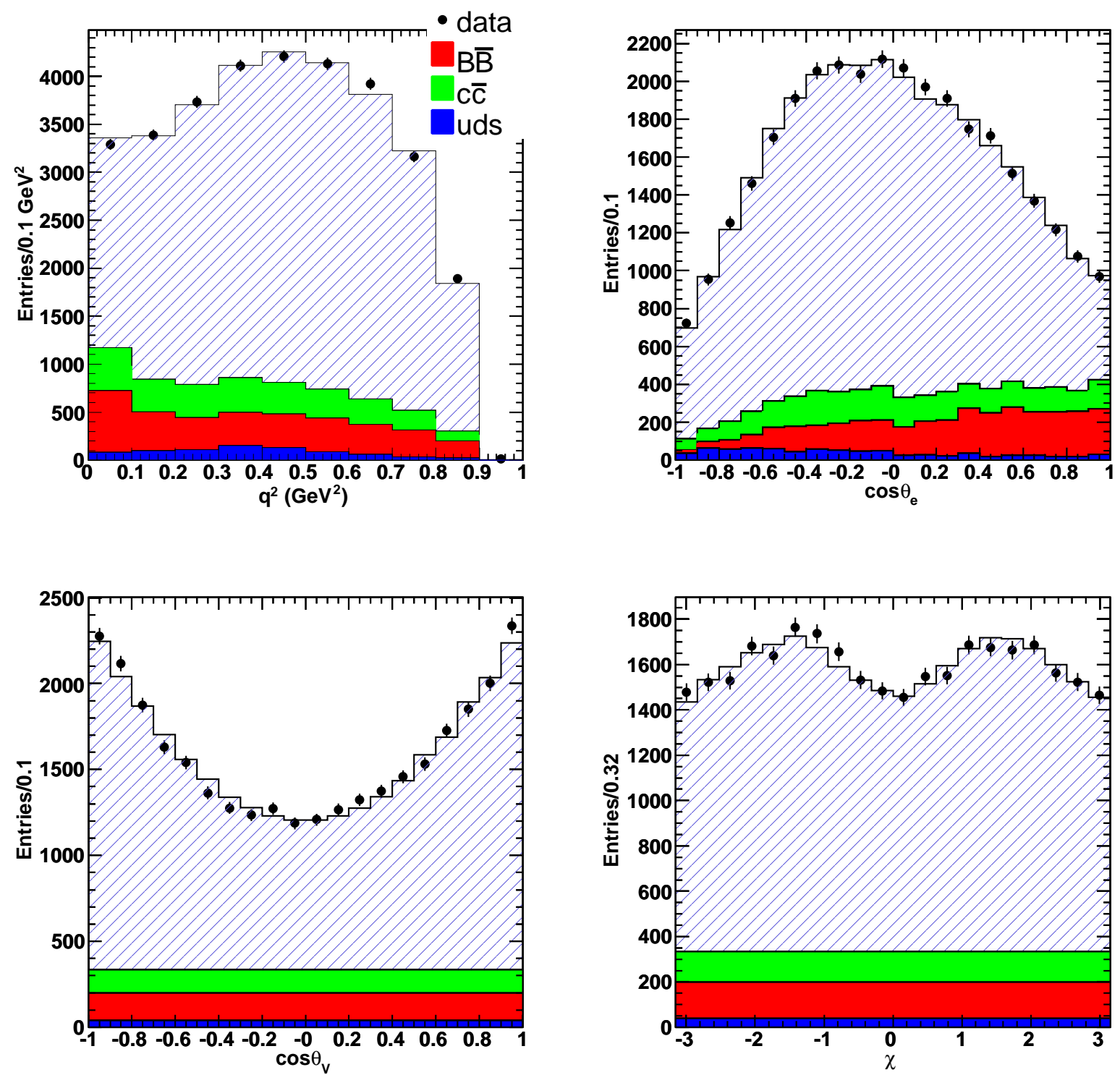

Figure 6.2: Projected distributions of the reconstructed values of the four kinematic variables which define the decay $D_{s}^{+} \rightarrow \phi e^{+} \nu_{e}$. Points with error bars correspond to real events. Here the distinction between $D_{s}^{+}$and $D_{s}^{-}$is not done. 


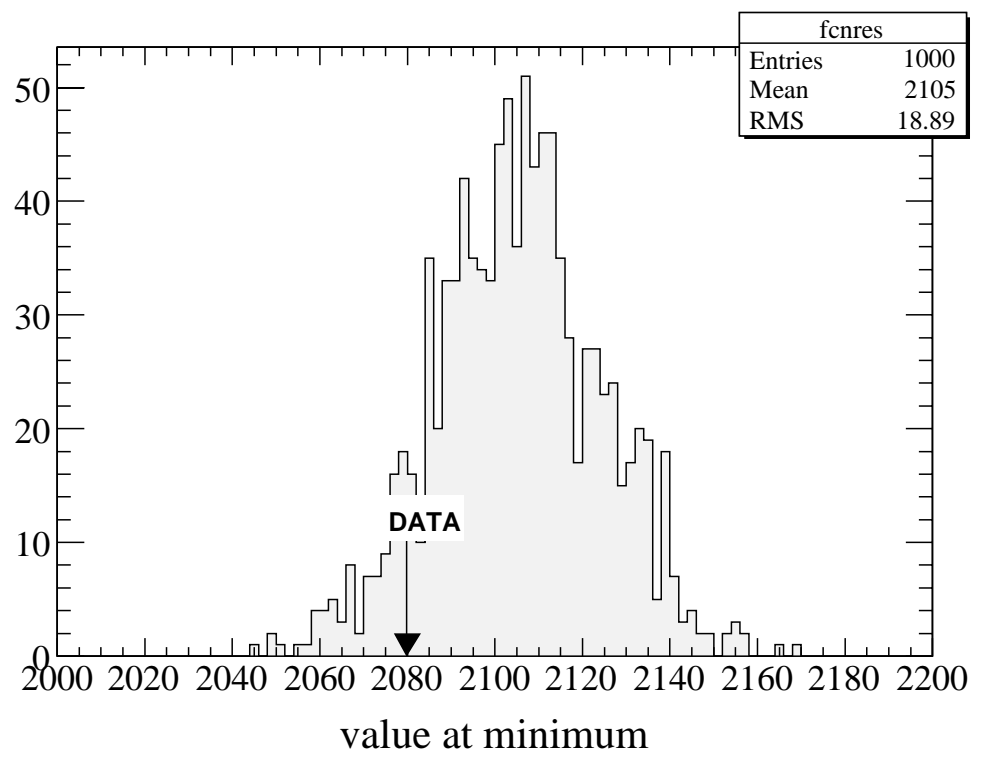

Figure 6.3: Values of the negative log-likelihood minimum obtained with 1000 toy experiments. The corresponding value measured with real data events has been indicated.

The value of the fitted parameter $\lambda_{A}$ corresponds to $m_{A}=1+\lambda_{A}^{2}=2.30_{-0.18}^{+0.24} \mathrm{GeV} / \mathrm{c}^{2}$.

It has been tried to fit, in addition, the value of the vector mass pole but the sensitivity to this parameter is too weak. The fit with 3 or 4 free parameters has been done fixing different values for $m_{V}$. The value at minimum for each fit is shown on Figure 6.4 (left side). This can be compared with the value at minimum for fits done with different values of $m_{A}$ (Figure 6.4, right side). The dotted lines correspond to a variation of $+1 / 2$ relative to the value at minimum. These plots illustrate clearly the difference of sensitivity to the two pole masses.

While $r_{2}$ and $\lambda_{A}$ are not really sensitive to the value of $m_{V}$, the fitted value of $r_{V}$ depends on $m_{V}$, as shown on Figure 6.5. We choose to fix $m_{V}$ at the value $2.1 \mathrm{GeV} / \mathrm{c}^{2}$ because it is compatible with data and it is the usual value used by the experiments measuring the form factors parameters.

In the parameterization used in this analysis, we consider that the two axial-vector form factors, $A_{1}\left(q^{2}\right)$ and $A_{2}\left(q^{2}\right)$, are governed by the same pole $\left(m_{A_{1}}=m_{A_{2}}=m_{A}\right)$. But more generally, they could have a different $q^{2}$ dependence. It has been tried to fit two independent pole masses for $A_{1}\left(q^{2}\right)$ and $A_{2}\left(q^{2}\right)$. We found that the sensitivity to $m_{A_{2}}$ is too weak, as shown on the maximum likelihood scan in Figure 6.6, and in the following we keep the same pole mass for these form factors. 

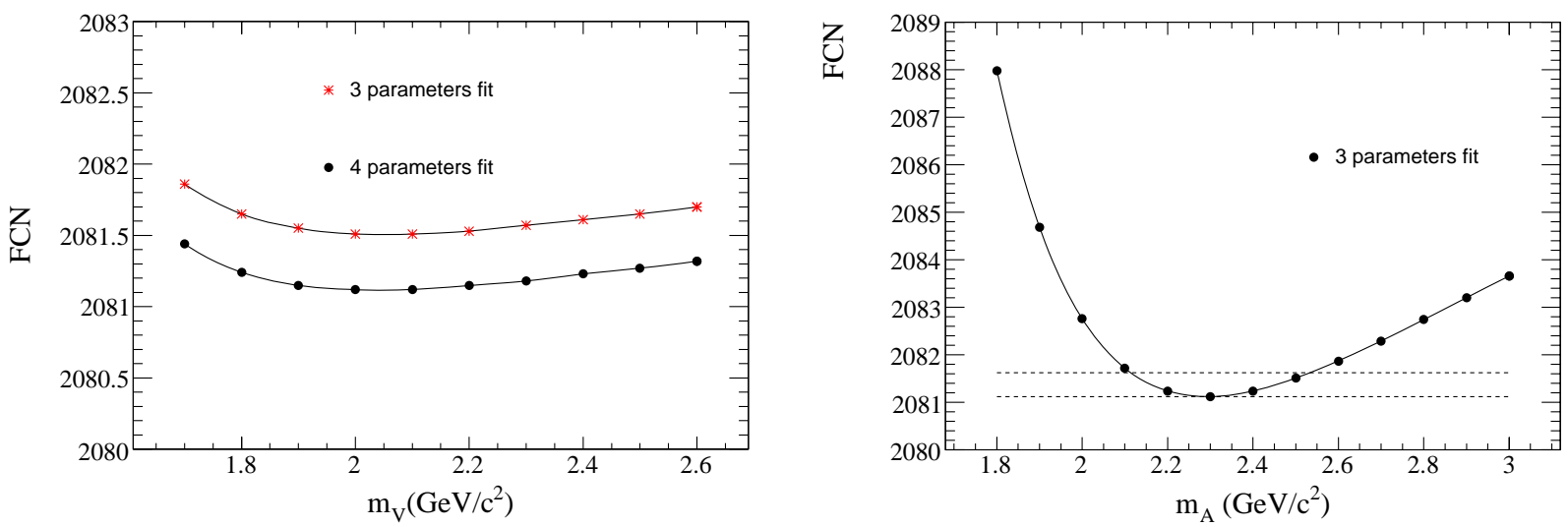

Figure 6.4: Left: value at minimum obtained as function of the vector mass pole value, for the fit fixing the axial-vector mass pole or not. Right: similar distribution obtaind by varying $m_{A}$, with $m_{V}$ fixed to $2.1 \mathrm{GeV} / \mathrm{c}^{2}$.

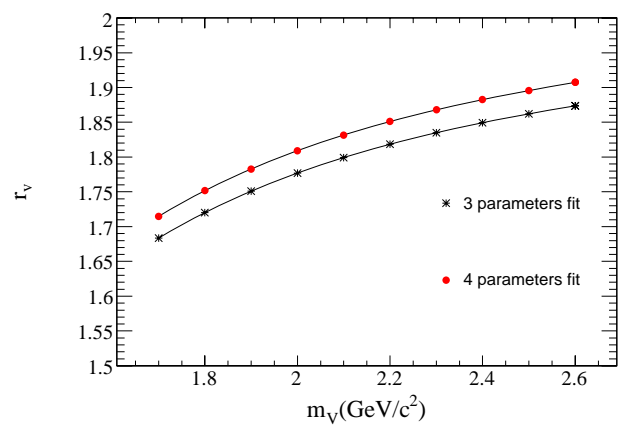

Figure 6.5: Fitted value of $r_{V}$ as function of $m_{V}$.

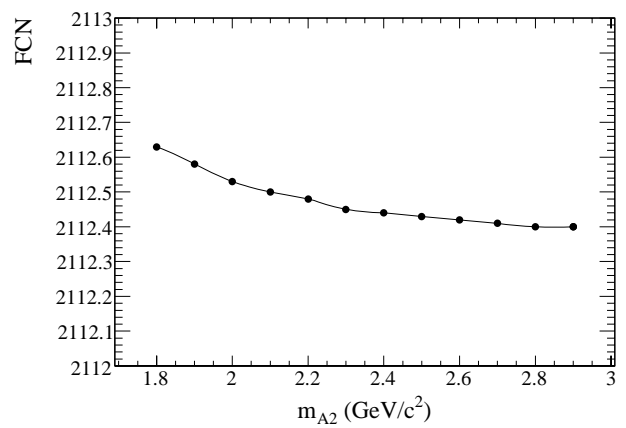

Figure 6.6: Value at minimum obtained as function of the axial-vector pole mass $m_{A 2}$. 


\subsection{Evidence for an $S$-wave contribution}

By analyzing the $D_{s} \rightarrow K^{+} K^{-} e^{+} \nu_{e}$ decay we measure an $S$-wave contribution through its interference with the $\phi$. This measurement is only sensitive to the $\mathrm{S}$-wave in a narrow mass window centered on $m_{\phi}$. Figure 6.7 shows the $K^{+} K^{-}$mass distribution when each event is weighted by $\cos \left(\theta_{V}\right)$. A signal is visible, which is not present in the simulation. We find an excess of weighted data events in the region between 1.01 and $1.03 \mathrm{GeV} / \mathrm{c}^{2}$ of $606 \pm 121$ events.

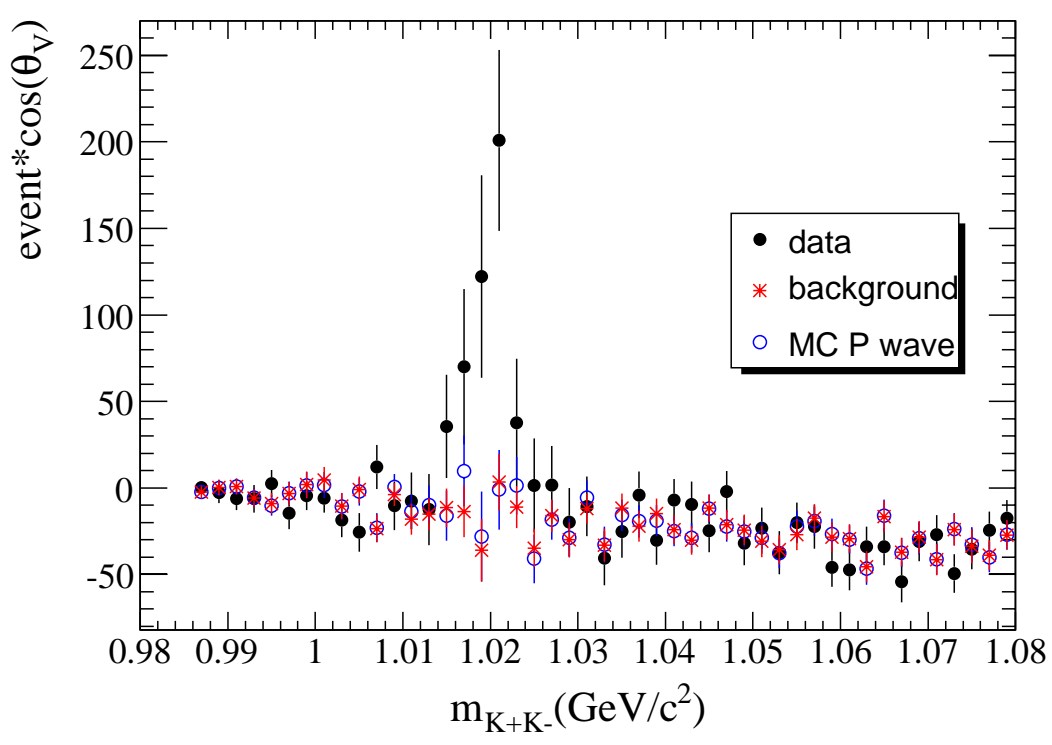

Figure 6.7: $K^{+} K^{-}$mass ditribution weighting each event by $\cos \left(\theta_{V}\right)$. Data are compared with the simulation where only the $P$ wave is present. $M C$ signal is scaled to the number of events fitted in data.

In principle, a forward-backward asymmetry originating from pure reconstruction effects (as difference of efficiencies between the $K^{-}$and $K^{+}$) could also be possible. Let's consider a $D_{s}^{+}$decaying semileptonically in the configuration shown in Figure 6.8-a. The final hadrons $K^{+}$and $K^{-}$have momentum $p^{+}$and $p^{-}$respectively, and $\cos \theta_{V}$ is positive. The efficiency to reconstruct this decay configuration is proportional to $\epsilon_{K^{+}}\left(p^{+}\right) \epsilon_{K^{-}}\left(p^{-}\right)$. We consider a second configuration, inverting the two kaons so that $\cos \theta_{V}$ is negative (the $K^{+}$has then momentum $p^{-}$, and the $K^{-}$has momentum $p^{+}$), as shown in Figure 6.8-b. In this case, the efficiency is proportional to $\epsilon_{K^{+}}\left(p^{-}\right) \epsilon_{K^{-}}\left(p^{+}\right)$. 
a)

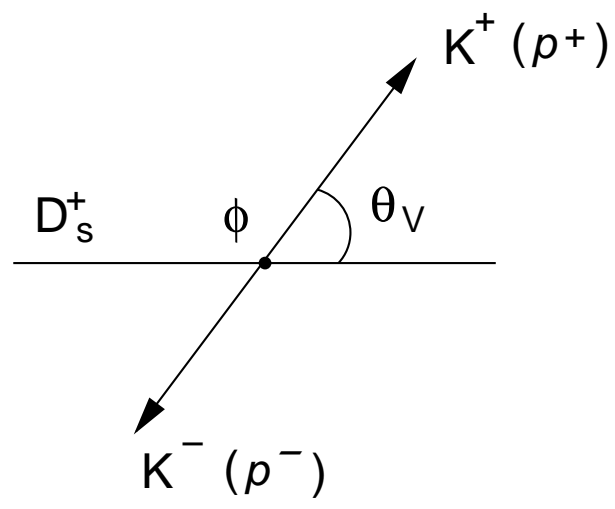

b)

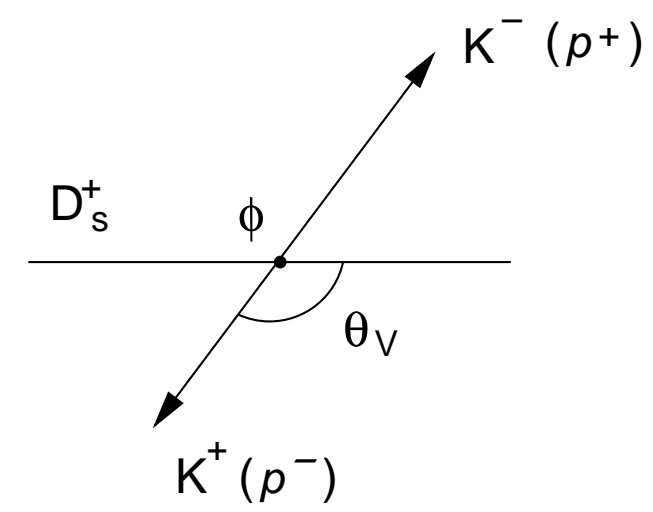

Figure 6.8: Decay of a $D_{s}^{+}$in two different configurations. $a$ : $\cos \theta_{V}$ is positive, $b$ : the $K^{+}$ and $K^{-}$have been inverted, $\cos \theta_{V}$ is negative.

We can write the asymmetry $A_{+-}$as:

$$
A_{+-}=\frac{\epsilon_{K^{+}}\left(p^{+}\right) \epsilon_{K^{-}}\left(p^{-}\right)-\epsilon_{K^{+}}\left(p^{-}\right) \epsilon_{K^{-}}\left(p^{+}\right)}{\epsilon_{K^{+}}\left(p^{+}\right) \epsilon_{K^{-}}\left(p^{-}\right)+\epsilon_{K^{+}}\left(p^{-}\right) \epsilon_{K^{-}}\left(p^{+}\right)} .
$$

For a given momentum, the kaon identification efficiency differs by a few percent for $K^{+}$ and $K^{-}$(see Figure 2.24): $\epsilon_{K^{+}}(p)=\epsilon_{K^{-}}(p)+\delta$, where $\delta>0$. The asymmetry can then be expressed as:

$$
A_{+-}=\frac{\delta\left(\epsilon_{K^{-}}\left(p^{-}\right)-\epsilon_{K^{-}}\left(p^{+}\right)\right)}{2 \epsilon_{K^{-}}\left(p^{+}\right) \epsilon_{K^{-}}\left(p^{-}\right)} .
$$

This quantity is, in principle, non-zero because $\delta$ is positive and the identification efficiency of kaons depends on the momentum. Considering the case of a $D_{s}^{-}$, to obtain its decay pattern, we have explained in Section 1.2.2 that the roles of the $K^{+}$and $K^{-}$have to be inverted. We should now obtain an asymmetry with opposite sign. Thus, on average, the asymmetry due to detector effects cancels. In data, separating the $D_{s}^{+}$and $D_{s}^{-}$as shown in Figure 6.9, we obtain a positive signal of the same order for both signs. This is expected for an $S$-wave contribution and not from a detector effect.

Using Equation 1.43, we fit, in addition to the form factors parameters, the value of $r_{0}$. The following results and correlation matrix are obtained:

$$
N_{S}=25162 \pm 177, r_{0}=15.0 \pm 2.5 \mathrm{GeV}^{-1}, \mathrm{r}_{\mathrm{V}}=1.836 \pm 0.047, \mathrm{r}_{2}=0.814 \pm 0.036
$$




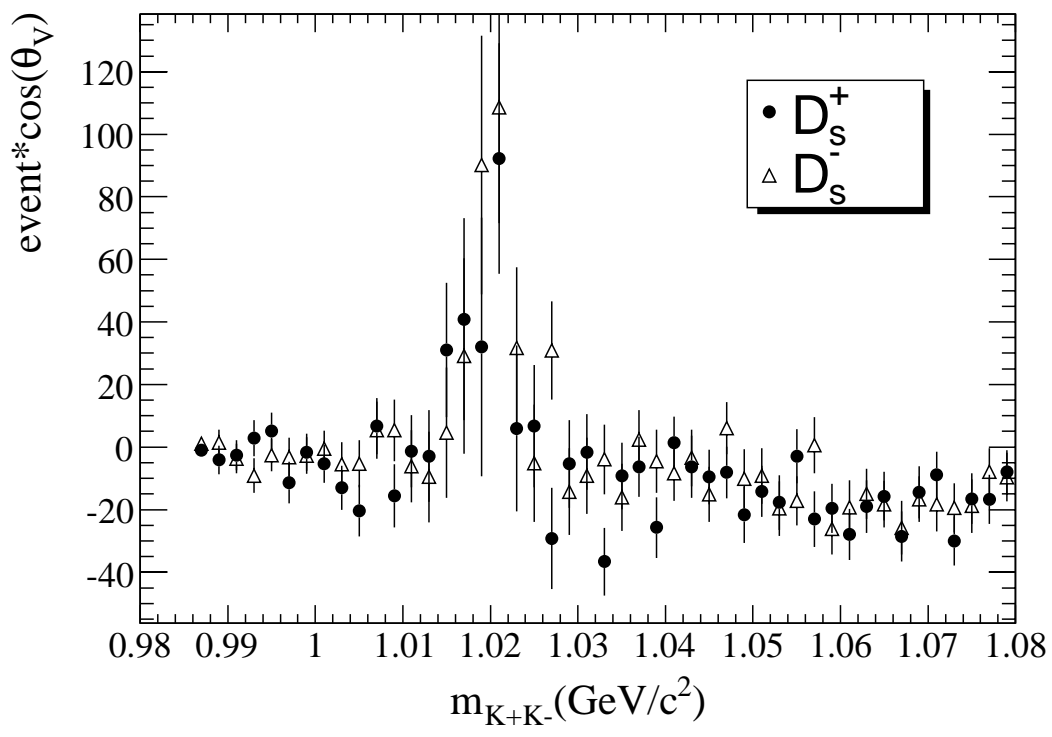

Figure 6.9: $K^{+} K^{-}$mass ditribution weighting each event by $\cos \left(\theta_{V}\right)$ as obtained with data. $D_{s}^{+}$and $D_{s}^{-}$are plotted separately.

$$
\left(\begin{array}{cccc}
1 & -0.015 & -0.062 & 0.001 \\
-0.015 & 1 & 0.043 & 0.002 \\
-0.062 & 0.043 & 1 & -0.150 \\
0.001 & 0.002 & -0.150 & 1
\end{array}\right)
$$

This result is shown on Figure 6.10, using the same binning as in Figure 6.2, and on Figure 6.11 using the same binning as adopted for the fit. To perform this fit, we use a constant average for the background in $\chi$, as it is expected to be flat (see Figure 6.10). Figure 6.12 shows the angular distributions for each of the five bins in $q^{2}$. We can see that the distributions resulting from the fit agree well with the data in each bin.

Fitting also the axial-vector pole mass, we obtain:

$$
\begin{aligned}
& \lambda_{A}=\left(1.14_{-0.08}^{+0.10}\right) \mathrm{GeV}^{1 / 2} / \mathrm{c}, \mathrm{N}_{\mathrm{S}}=25152 \pm 177, \mathrm{r}_{0}=15.3 \pm 2.6 \mathrm{GeV}^{-1} \\
& r_{V}=1.868 \pm 0.061, r_{2}=0.763 \pm 0.072
\end{aligned}
$$

and the correlation matrix:

$$
\left(\begin{array}{ccccc}
1 & -0.074 & 0.134 & 0.624 & -0.858 \\
-0.074 & 1 & -0.046 & -0.095 & 0.064 \\
0.134 & -0.046 & 1 & 0.118 & -0.114 \\
0.624 & -0.095 & 0.118 & 1 & -0.595 \\
-0.858 & 0.064 & -0.114 & -0.595 & 1
\end{array}\right)
$$



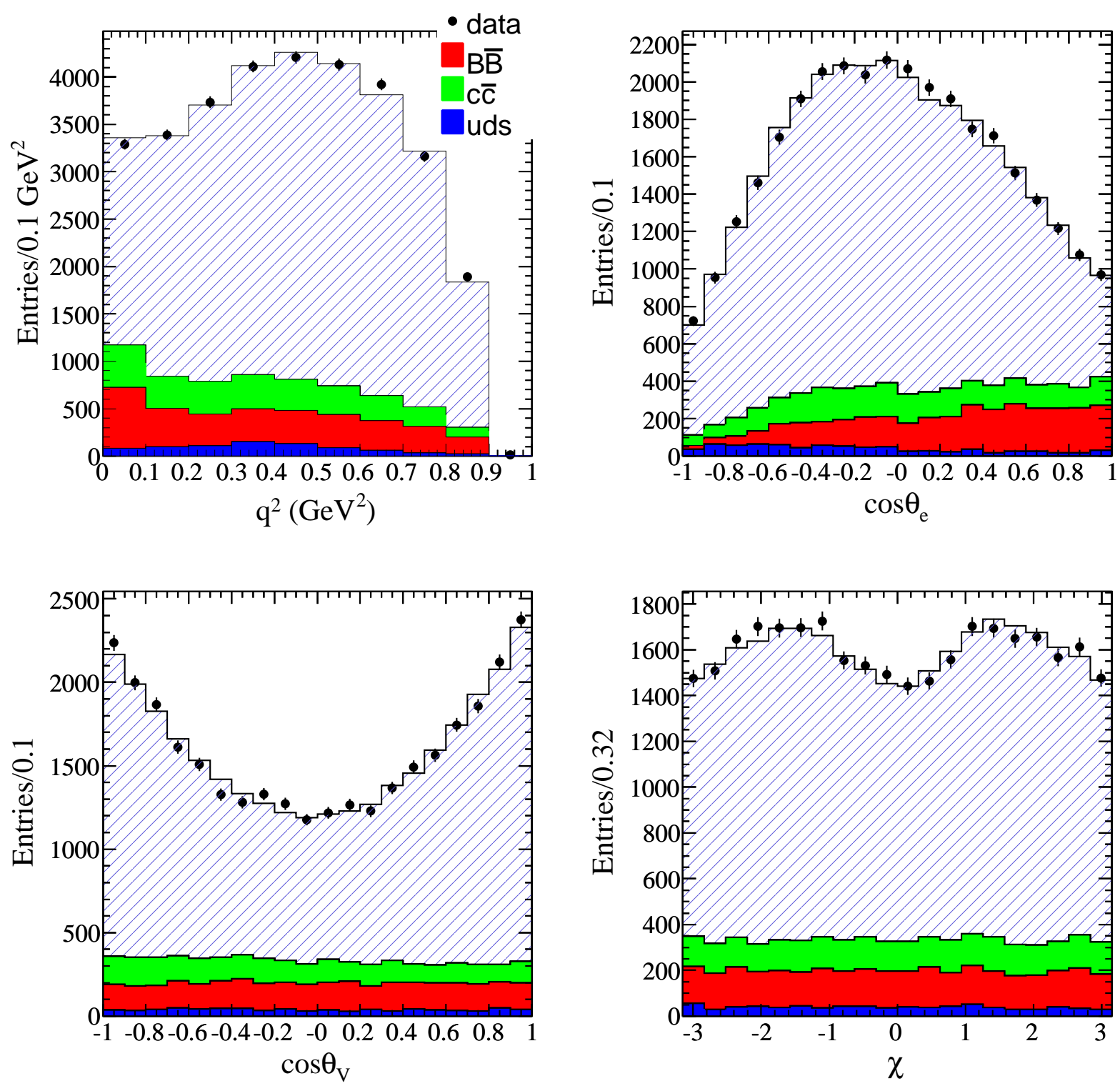

Figure 6.10: Projected distributions of the reconstructed values of the four kinematic variables which define the decay $D_{s}^{+} \rightarrow \phi e^{+} \nu_{e}$. Points with error bars correspond to real events. Hatched histograms show the fit result when taking into account the asymmetry. 

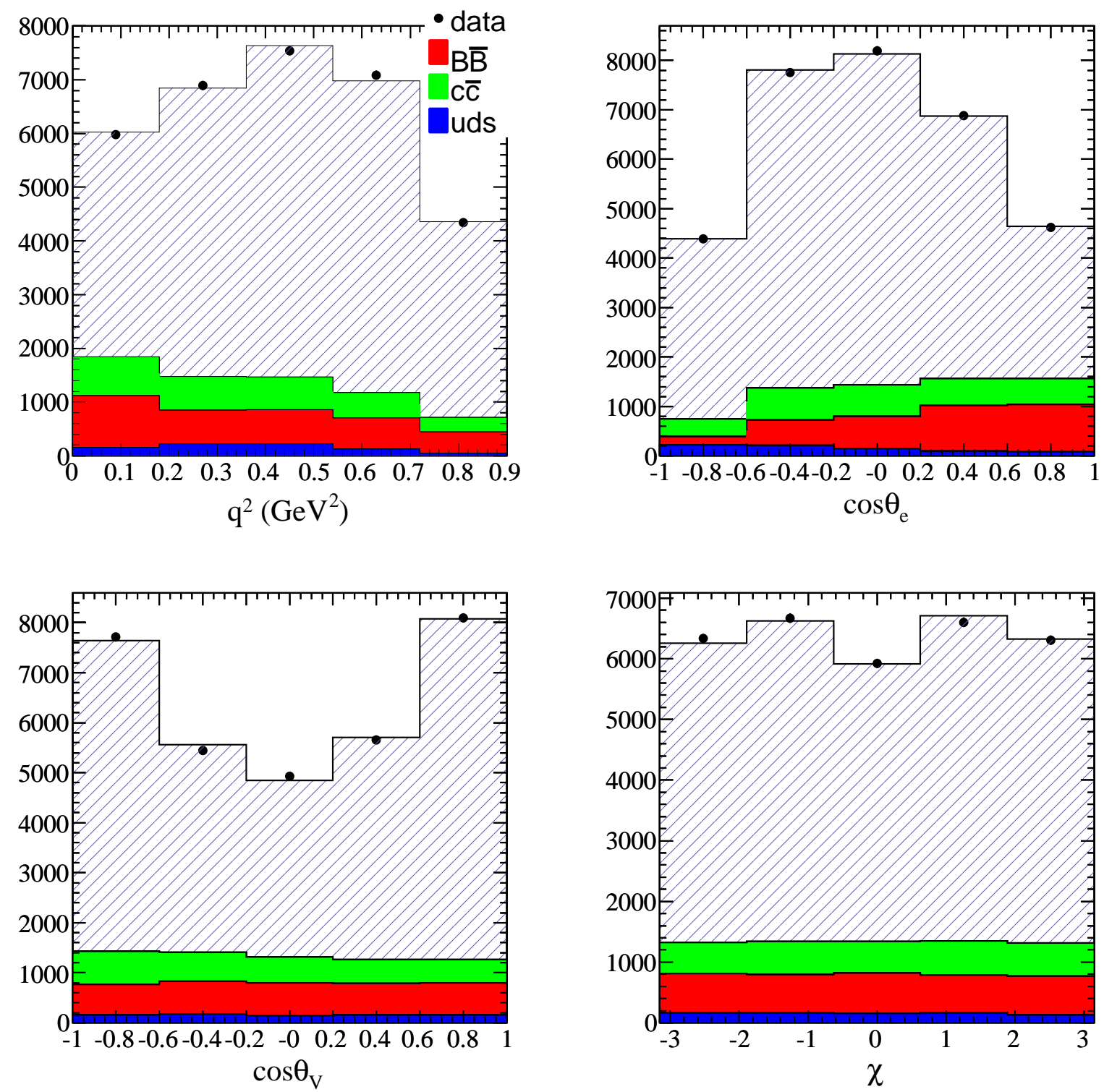

Figure 6.11: Projected distributions of the reconstructed values of the four kinematic variables which define the decay $D_{s}^{+} \rightarrow \phi e^{+} \nu_{e}$. Points with error bars correspond to real events. Hatched histograms show the fit result when taking into account the asymmetry. The binning is the same as used in the fit. 

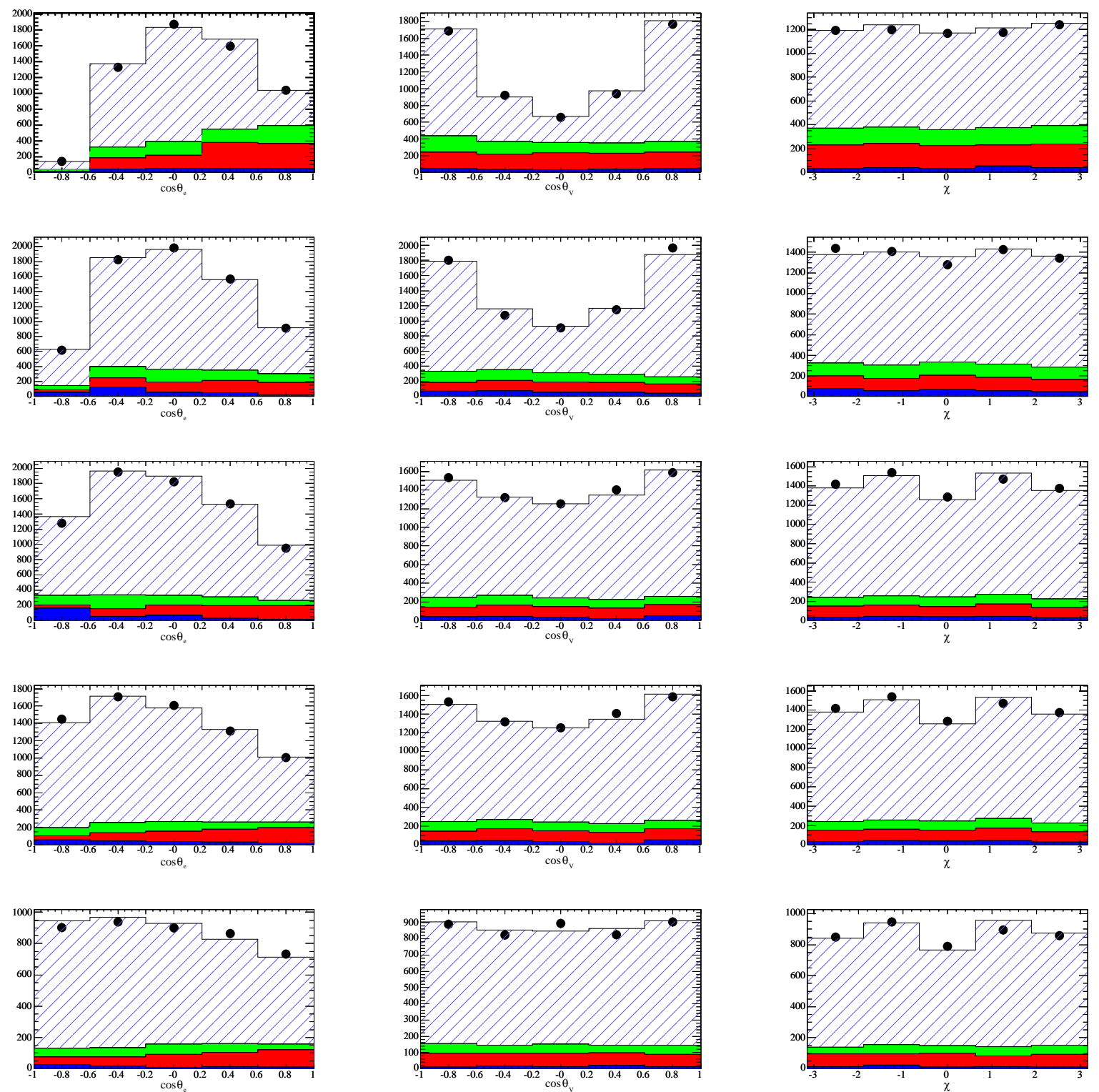

Figure 6.12: Projected distributions of the angular variables in each $q^{2}$ bin. From left to right: $\cos \theta_{l}, \cos \theta_{V}$ and $\chi$. Each line corresponds to a $q^{2}$ bin. 
The comparison between data and simulation weighted using the fitted value $r_{0}=$ 15.3 $\mathrm{GeV}^{-1}$ is shown on Figure 6.13.

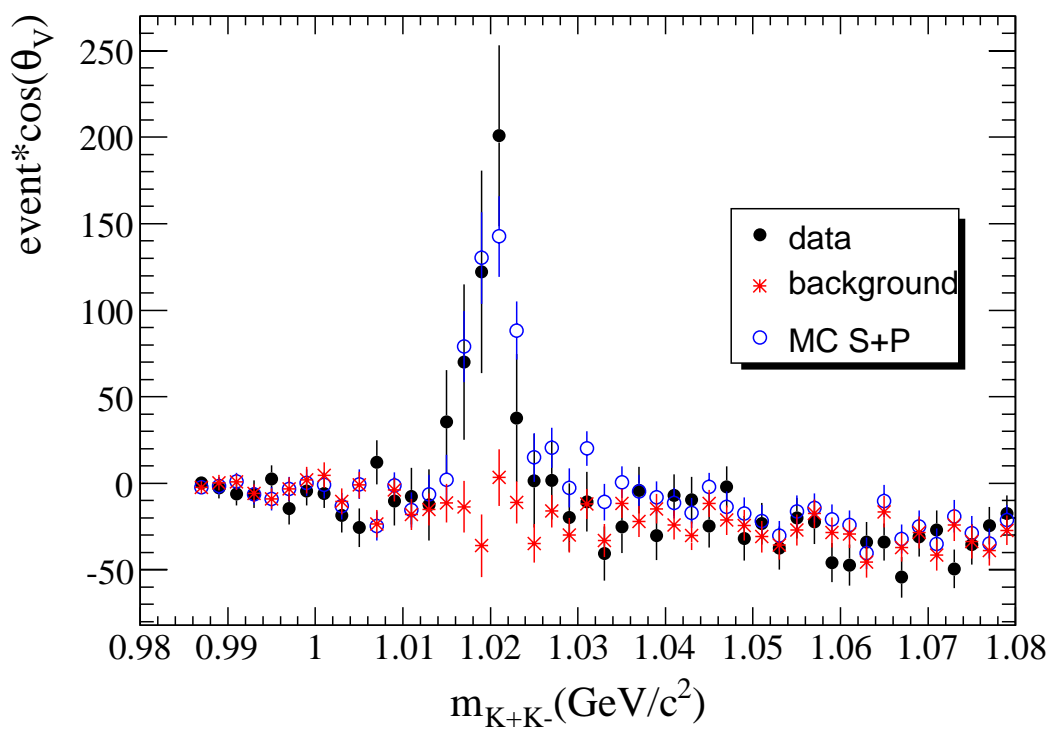

Figure 6.13: $K^{+} K^{-}$mass ditribution weighting each event by $\cos \left(\theta_{V}\right)$. Signal $M C$ has been reweighted to include an $S$-wave component with the parameter $r_{0}=15.3 \mathrm{GeV}^{-1}$.

The fitted value of $r_{0}=15.3 \mathrm{GeV}^{-1}$ corresponds to the ratio (see Figure 6.14 ):

$$
\frac{B R\left(D_{s} \rightarrow f_{0} e^{+} \nu_{e}\right) \times B R\left(f_{0} \rightarrow K^{+} K^{-}\right)}{B R\left(D_{s} \rightarrow K^{+} K^{-} e^{+} \nu_{e}\right)}=0.23_{-0.08}^{+0.12 \%}
$$

within the selected $K^{+} K^{-}$mass interval between 1.01 and $1.03 \mathrm{GeV} / \mathrm{c}^{2}$. This mass interval contains 0.131 of the total $f_{0} \rightarrow K^{+} K^{-}$decay spectrum. 


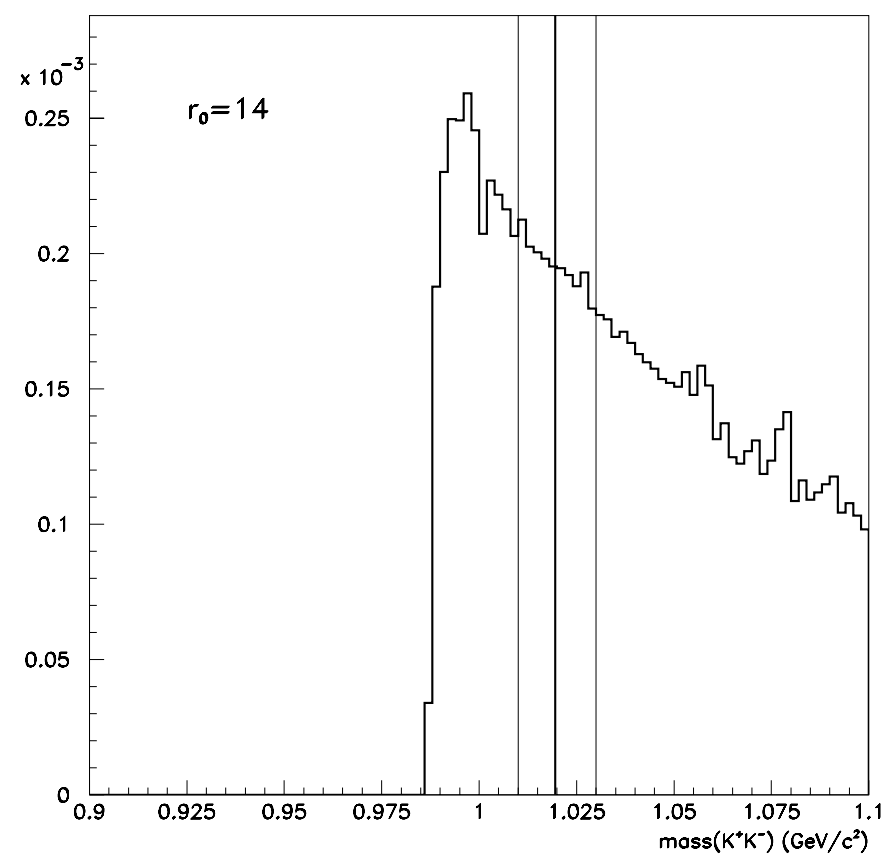

Figure 6.14: Fraction of the $S$-wave component per event in $2 \mathrm{MeV} / \mathrm{c}^{2}$ bins, as a function of the $K^{+} K^{-}$mass, in the decay $D_{s} \rightarrow K^{+} K^{-} e^{+} \nu_{e}$. 


\subsection{Fit stability}

In order to test the fit stability, we have performed the fit for different cut values on the Fisher discriminant against $c \bar{c}$ events. The number of candidates selected in data and estimated background are summarized in Table 6.1, with the corresponding fitted parameters for three different cuts on the Fisher discriminant. The cut value at 0 is used for the reference fit. We can see that the variation from 0.3 to -0.3 corresponds to an increase of the number of signal by a factor two, and an increase of the background events by a factor 4.6.

The fit results, when the axial-vector pole mass is also fitted are summarized in Table 6.2 for the same cut values.

\begin{tabular}{|c|c|c|c|c|c|}
\hline$F_{c c}$ cut & data & background & fitted signal & $r_{2}$ & $r_{V}$ \\
\hline-0.3 & 40647 & 11279 & $29386 \pm 200$ & $0.778 \pm 0.035$ & $1.855 \pm 0.046$ \\
\hline 0 & 31839 & 6675 & $25157 \pm 177$ & $0.807 \pm 0.036$ & $1.826 \pm 0.047$ \\
\hline 0.3 & 18297 & 2474 & $15800 \pm 135$ & $0.833 \pm 0.043$ & $1.736 \pm 0.054$ \\
\hline
\end{tabular}

Table 6.1: Number of candidates in data, estimated background, and fit results for different cut values on the Fisher discriminant $F_{c c}$.

\begin{tabular}{|c|c|c|c|c|}
\hline$F_{c c}$ cut & fitted signal & $r_{2}$ & $r_{V}$ & $m_{A}$ \\
\hline-0.3 & $29357 \pm 199$ & $0.671 \pm 0.074$ & $1.923 \pm 0.063$ & $2.14_{-0.14}^{+0.18}$ \\
\hline 0 & $25144 \pm 177$ & $0.755 \pm 0.071$ & $1.855 \pm 0.061$ & $2.28_{-0.18}^{+0.23}$ \\
\hline 0.3 & $15792 \pm 135$ & $0.745 \pm 0.084$ & $1.785 \pm 0.069$ & $2.19_{-0.17}^{+0.22}$ \\
\hline
\end{tabular}

Table 6.2: Fit results when the axial-vector pole mass is also fitted, for different cut values on the Fisher discriminant $F_{c c}$. The number of candidates in data and estimated background are the same as in Table 6.1.

The fit results for these three different cuts are shown on Figure 6.15.

The three samples are not independent and to have a more relevant comparison, the systematics errors should have been computed for the three cut values. Our nominal measurement with the five parameters fit is stable. 

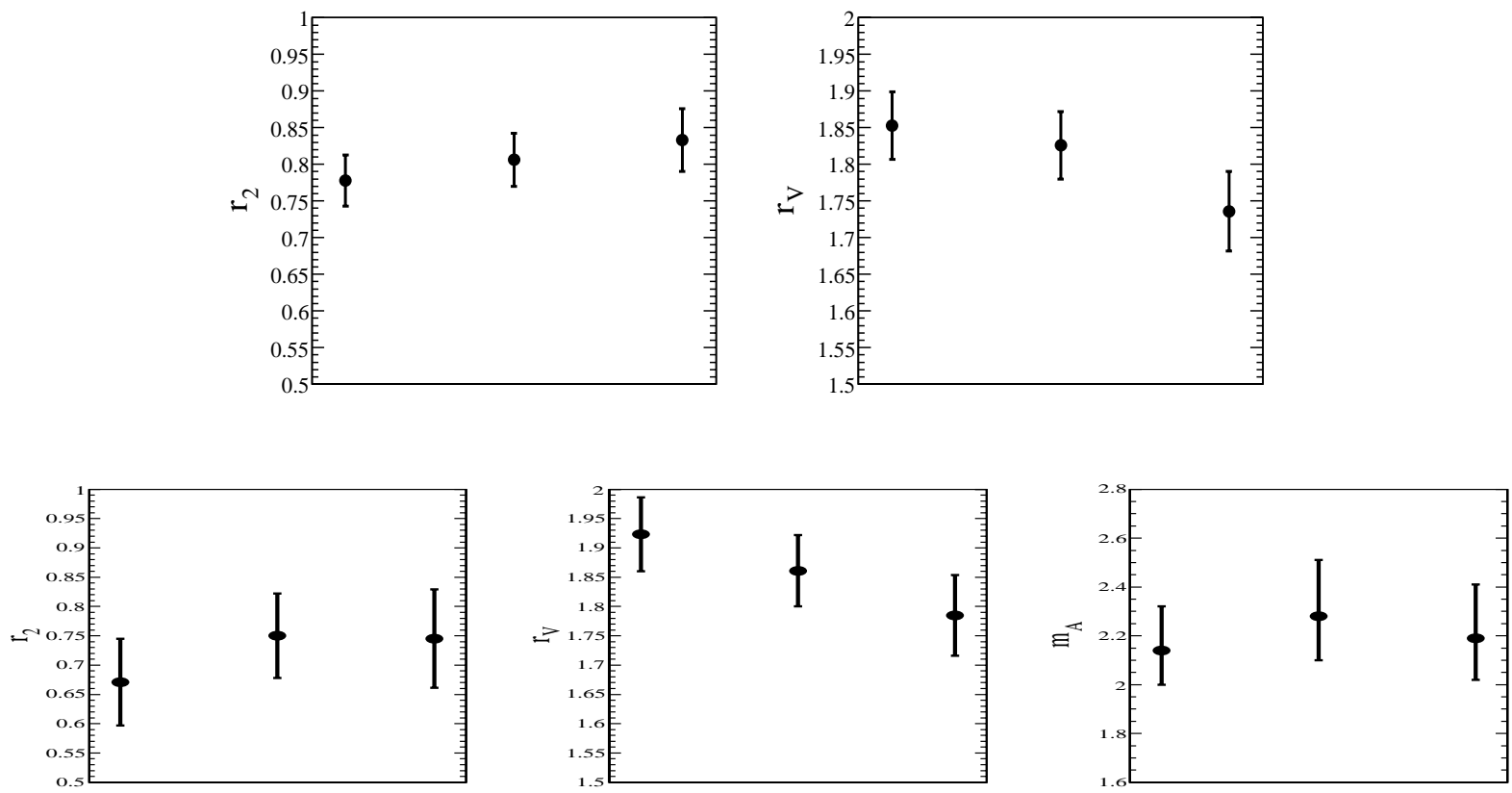

Figure 6.15: Fit result for different cuts on $F_{c c}$, from left to right: $-0.3,0,0.3$. The upper plots show the fitted values of $r_{2}$ and $r_{V}$. Bottom plots show fit results with the axial-vector pole mass as an additional free parameter. 


\section{Chapter 7}

\section{Systematic uncertainties}

'I quite agree with you,' said the Duchess; 'and the moral of that is:" be what you wouls seem to be" - or if you'd like it put more simply-" never imagine yourself not to be otherwise than what it might appear to others that what you were or might have been was not otherwise than what you had been would have appeared to them to be otherwise."

In this chapter, we detail the different sources of systematic uncertainties. Most of them originate from corrections that are used to improve the agreement between data and simulation. Remaining uncertainties on these corrections correspond to systematic errors on the fitted parameters. We quote the corresponding variation on the fitted parameters with the convention :

$$
\delta\left(\lambda_{k}\right)=\lambda_{k}^{\text {varied }}-\lambda_{k}^{\text {not varied }}
$$

where $\lambda_{k}^{\text {not varied }}$ corresponds to the nominal value of a parameter used to fit the data. For each systematics evaluation, we give the errors for the four parameters fit $\left(N, r_{2}, r_{V}\right.$ and $\left.r_{0}\right)$, and the errors for the fit where the axial-vector mass pole is also fitted. We evaluate separately the contributions of remaining uncertainties on signal and background estimates, and the PID corrections. The corresponding variations $\delta\left(\lambda_{k}\right)$ are quoted at the end of each subsection. Finally, all systematic uncertainties have been collected in Tables 7.9 and 7.10. 


\subsection{Signal Monte Carlo corrections}

As noted in section 4.1.1, the agreement between real and simulated events on $D_{s}^{+}$production characteristics is rather good after having applied several weights defined from the fragmentation distribution, the spectator system momentum, and $R_{2}$. The remaining differences between data and MC after these corrections can be seen in the Fisher variables for PHIPI events in Figure 7.1. We define an additional weight as function of $F_{b b}$ which corresponds to the ratio of the normalized distributions of $F_{b b}$ in data and in MC for PHIPI events. This correction is used to evaluate the systematic uncertainty related to the generator tuning on signal event.
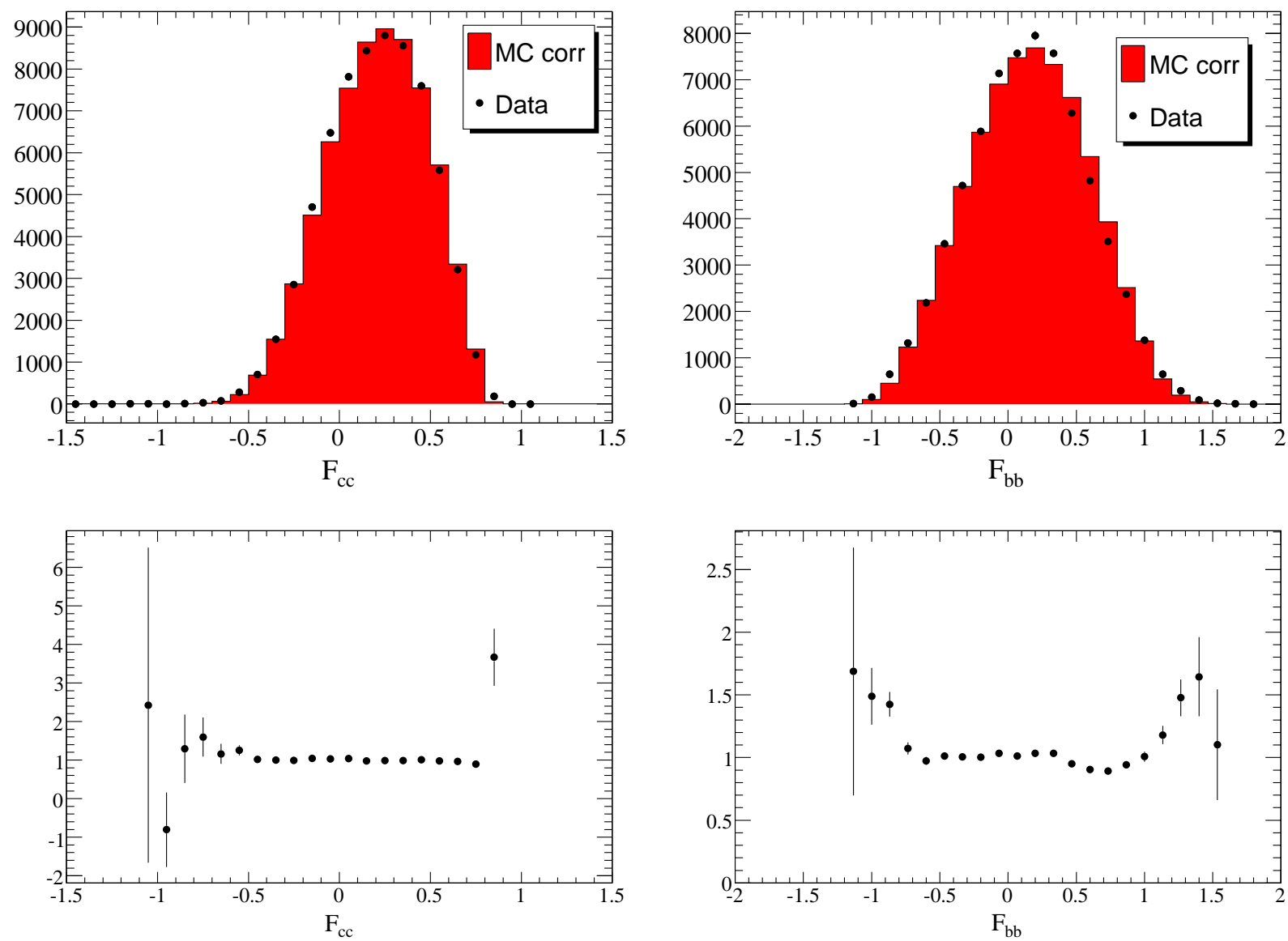

Figure 7.1: Data/MC comparison for the two Fisher distributions in PHIPI events after having applied the corrections defined in section 4.1.1.

The second systematic uncertainty of the MC signal involves determination of the $D_{s}^{+}$ direction and of the missing energy. The data/MC agreement for the $D_{s}^{+}$angles $\left(\theta_{D s}\right.$ and $\left.\phi_{D s}\right)$ has been found to be satisfactory using PHIPI events (Figure 4.11). Nevertheless to 
evaluate the small remaining differences, a smearing has been added on the $D_{s}^{+}$angles. The smearing is done by adding to the angle value, for each event, a random number generated according to a Gaussian distribution. The effect of this smearing is shown on Figure 7.2.
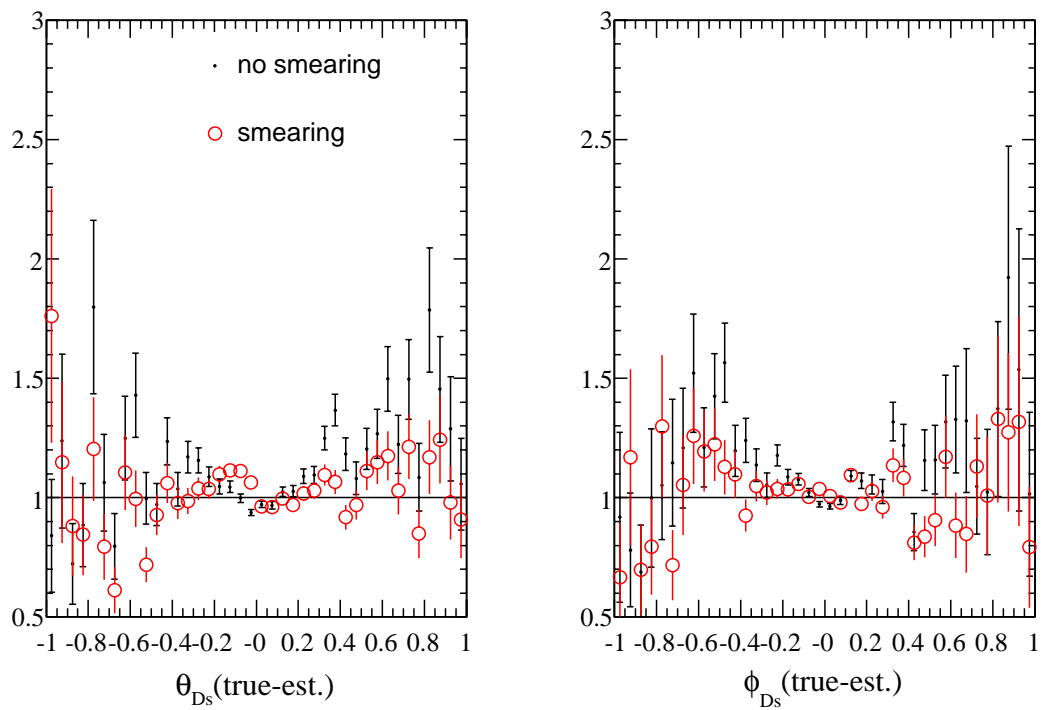

Figure 7.2: Difference between $D_{s}^{+}$angles obtained from the decay products $(\phi \pi)$ and from the other particles in the event. The red dots are obtained after having added a smearing.

In the same way, to evaluate the uncertainty on the missing energy, we change the smearing defined in Table 4.1, using Gaussian distribution with sigma of $100 \mathrm{MeV}$ and 170 $\mathrm{MeV}$ for each interval in the missing energy measured in the opposite hemisphere. This procedure changes the resolution on the missing energy. The effect of these corrections are shown on figure 7.3 .

The effect of the different corrections related to the signal MC on the fitted parameters are summarized in Tables 7.1 and 7.2.

\begin{tabular}{|l|c|c|c|c|}
\hline corrections & $\delta\left(N_{s}\right)$ & $\delta\left(r_{0}\right)$ & $\delta\left(r_{V}\right)$ & $\delta\left(r_{2}\right)$ \\
\hline Fisher variables for signal MC & +1 & 0 & -0.012 & -0.011 \\
smearing $D_{s}^{+}$angles & +10 & -0.1 & -0.001 & -0.005 \\
smearing missing energy $(100 \mathrm{MeV})$ & -2 & +0.1 & 0 & -0.001 \\
smearing missing energy $(170 \mathrm{MeV})$ & +3 & -0.1 & -0.001 & +0.004 \\
\hline
\end{tabular}

Table 7.1: Variations of fitted parameters: $N_{s}, r_{0}\left(\right.$ in $\left.\mathrm{GeV}^{-1}\right), r_{2}, r_{V}$, for different corrections applied on simulated signal events. 


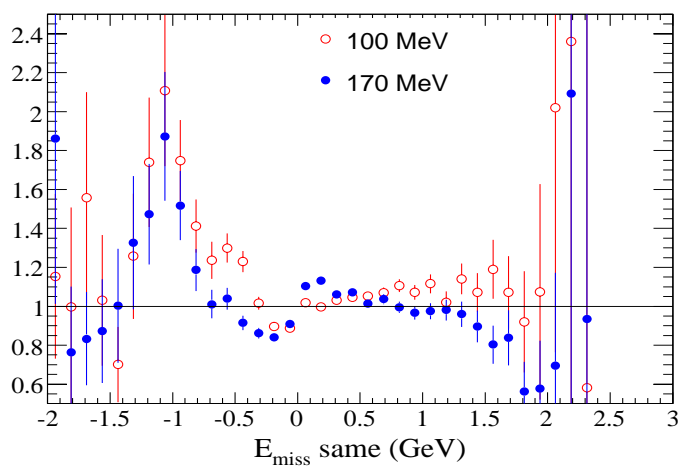

Figure 7.3: Data/MC missing energy ratio when a smearing is applied on the missing energy in the candidate hemisphere. These distributions are obtained with PHIPI events.

\begin{tabular}{|l|c|c|c|c|c|}
\hline corrections & $\delta\left(N_{s}\right)$ & $\delta\left(r_{0}\right)$ & $\delta\left(r_{V}\right)$ & $\delta\left(r_{2}\right)$ & $\delta\left(\lambda_{A}\right)$ \\
\hline Fisher variables for signal MC & -1 & -0.1 & -0.013 & -0.009 & 0 \\
smearing $D_{s}^{+}$angles & +10 & 0 & -0.001 & -0.005 & 0 \\
smearing missing energy $(100 \mathrm{MeV})$ & +5 & 0 & -0.009 & +0.013 & +0.02 \\
smearing missing energy $(170 \mathrm{MeV})$ & +2 & -0.1 & +0.003 & -0.003 & -0.01 \\
\hline
\end{tabular}

Table 7.2: Variations of fitted parameters: $N_{s}, r_{0}$ (in $\mathrm{GeV}^{-1}$ ), $r_{2}, r_{V}$ and $\lambda_{A}$ (in $\left.\left(\mathrm{GeV} / \mathrm{c}^{2}\right)^{1 / 2}\right)$, for different corrections applied on simulated signal events.

\subsection{Background Monte Carlo corrections}

The different systematic uncertainties due to the background Monte Carlo corrections are detailed in the next subsections. The variations of the fitted parameters are summarized in the last part in Tables 7.3 and 7.4.

\subsubsection{Fragmentation correction on the $c \bar{c}$ background}

As explained in Section 4.3.2, we apply a correction on $c \bar{c}$ background events to correct the c-quark fragmentation process in simulation. The correction is different for events with a non-strange $D$ meson and events with a $D_{s}^{+}$meson. For the uncertainty on $D_{s}^{+}$events, we define an additional weight as function of $F_{b b}$, in the same way as we do for the evaluation of the signal MC uncertainty. For events with a $D$ meson, the uncertainty on the correction is mainly coming from the selected $D$ momentum range within which these corrections are obtained (see Figure 4.30). To take this effect into account, we determine an additional weight depending on the multiplicity using a first order polynomial expression leading to a 
typical variation of $10 \%$ of the correction over its range.

To give an idea of the global effect of the correction on the $c \bar{c}$ background, the fit has been done without any correction from fragmentation. The following variations have been found:

$$
\begin{gathered}
\delta\left(N_{s}\right)=-525, \delta\left(r_{0}\right)=+0.3, \delta\left(r_{V}\right)=-0.017, \delta\left(r_{2}\right)=-0.012 \\
\delta\left(N_{s}\right)=-537, \delta\left(r_{0}\right)=+0.7, \delta\left(r_{V}\right)=-0.053, \delta\left(r_{2}\right)=-0.068, \delta\left(\lambda_{A}\right)=-0.07
\end{gathered}
$$

The estimated uncertainty on this correction is of the order of $10 \%$ of its global effect.

\subsection{2 $B \bar{B}$ background correction}

The uncertainty of this correction is mainly due to the subtraction of off-peak events from on-peak events. To evaluate the uncertainty on the normalization of one sample relatively to the other, we count the number of $D_{s}^{+}$candidate in both samples for $x_{D s}>0.44$. We find that the uncertainty on the normalization is equivalent to a change of $2 \%$ of the luminosity of the off-peak dataset. Using this normalization, we define a new parameterization as function of $R_{2}$ and the multiplicity, that we use to evaluate the uncertainty on the $B \bar{B}$ background correction.

\subsubsection{Continuum background under the $\phi$ meson}

As it has been explained in section 4.3.5, two components of the continuum background MC are rescaled: $c \bar{c}$ events and light quark events. As it can be seen on Figure 4.40, the remaining difference on the rate of the continuum background is about $10 \%$. To evaluate the systematic uncertainty on the continuum background, we use a global rescaling of $10 \%$ in addition to the corrections defined for $c \bar{c}$ events and light quark events.

\subsubsection{Peaking background correction}

As explained is Section 4.3.4, different components of the charm peaking background are rescaled. For events with a $\phi$ meson from fragmentation, we use $1.0 \pm 0.2$. For events with a $\phi$ meson coming from a charm hadron, we have determined a scaling factor of $0.8 \pm 0.1$. For events with a $\phi$ meson produced in light quark events, we have determined a scaling factor of $1.0 \pm 0.2$. The uncertainty on $\phi$ mesons coming from b-hadrons has been taken into account in the uncertainty on the $B \bar{B}$ background correction. 


\subsubsection{Summary of background MC corrections systematics}

Systematic uncertainties from background MC corrections are summarized in Tables 7.3 and 7.4 .

\begin{tabular}{|l|c|c|c|c|}
\hline corrections & $\delta\left(N_{s}\right)$ & $\delta\left(r_{0}\right)$ & $\delta\left(r_{V}\right)$ & $\delta\left(r_{2}\right)$ \\
\hline Fragmentation correction on $c \bar{c}$ background & +37 & -0.1 & -0.003 & 0 \\
$B \bar{B}$ background correction & -230 & 0 & +0.033 & +0.002 \\
continuum bkg. & -198 & +0.5 & +0.018 & 0 \\
$\phi$ from fragmentation & -118 & 0 & -0.008 & -0.003 \\
$\phi$ from c-hadrons & -77 & 0 & -0.004 & -0.002 \\
$\phi$ from light quark events & -95 & 0 & -0.006 & -0.002 \\
\hline
\end{tabular}

Table 7.3: Variations of fitted parameters: $N_{s}, r_{0}\left(\right.$ in $\left.\mathrm{GeV}^{-1}\right), r_{2}, r_{V}$, for different corrections applied on simulated background events.

\begin{tabular}{|l|c|c|c|c|c|}
\hline corrections & $\delta\left(N_{s}\right)$ & $\delta\left(r_{0}\right)$ & $\delta\left(r_{V}\right)$ & $\delta\left(r_{2}\right)$ & $\delta\left(\lambda_{A}\right)$ \\
\hline Fragmentation corr. on $c \bar{c}$ back. & +38 & -0.1 & -0.007 & +0.004 & +0.01 \\
$B \bar{B}$ background correction & -236 & +0.1 & +0.050 & -0.024 & -0.04 \\
continuum bkg. & -202 & +0.6 & +0.032 & +0.023 & -0.03 \\
$\phi$ from fragmentation & -120 & -0.1 & -0.019 & +0.013 & +0.03 \\
$\phi$ from c-hadrons & -80 & +0.1 & -0.002 & +0.013 & -0.02 \\
$\phi$ from light quark events & -100 & +0.1 & +0.007 & -0.024 & -0.03 \\
\hline
\end{tabular}

Table 7.4: Variations of fitted parameters: $N_{s}, r_{0}$ (in $\mathrm{GeV}^{-1}$ ), $r_{2}, r_{V}$ and $\lambda_{A}$ (in $\left.\left(\mathrm{GeV} / \mathrm{c}^{2}\right)^{1 / 2}\right)$, for different corrections applied on simulated background events.

\subsection{Fitting procedure}

This source of uncertainties corresponds to the effects of finite Monte-Carlo statistics which have been studied in Section 5.4.1. Results have been given in Equation 5.6, and we evalutate the systematic uncertainty to be $0.46 * \sigma_{f i t}$, where $\sigma_{f i t}$ is the statistical error obtained in the fit.

\subsection{Particle identification efficiencies}

Effects induced by momentum dependent differences on the electron and charged kaon reconstruction efficiency between real and simulated events are evaluated. Standard correction 
factors determined in BABAR are applied to correct for these differences which are typically of a few percent (see Section 2.3). Differences in efficiencies for neutral particles are also corrected. Fitted values of the parameters measured in the present analysis are quoted after applying these corrections. The corresponding systematic uncertainties are estimated by turning off these corrections for the signal, separately for the PID and for neutral particles.

\begin{tabular}{|l|c|c|c|c|}
\hline corrections & $\delta\left(N_{s}\right)$ & $\delta\left(r_{0}\right)$ & $\delta\left(r_{V}\right)$ & $\delta\left(r_{2}\right)$ \\
\hline PID efficiencies & +3 & -0.6 & +0.001 & +0.019 \\
Neutrals corrections & +1 & -0.1 & -0.009 & -0.002 \\
\hline
\end{tabular}

Table 7.5: Variations of fitted parameters: $N_{s}, r_{0}\left(\right.$ in $\left.\mathrm{GeV}^{-1}\right), r_{2}, r_{V}$.

\begin{tabular}{|l|c|c|c|c|c|}
\hline corrections & $\delta\left(N_{s}\right)$ & $\delta\left(r_{0}\right)$ & $\delta\left(r_{V}\right)$ & $\delta\left(r_{2}\right)$ & $\delta\left(\lambda_{A}\right)$ \\
\hline PID efficiencies & +1 & -0.6 & +0.006 & +0.011 & +0.01 \\
Neutrals corrections & -5 & -0.2 & -0.018 & +0.012 & +0.02 \\
\hline
\end{tabular}

Table 7.6: Variations of fitted parameters: $N_{s}, r_{0}$ (in $\left.\mathrm{GeV}^{-1}\right), r_{2}, r_{V}$ and $\lambda_{A}$ (in $\left.\left(\mathrm{GeV} / \mathrm{c}^{2}\right)^{1 / 2}\right)$.

\subsection{Radiative events}

In this analysis, radiative events, where a photon is emitted in the initial or final state, are treated in the same way as non radiative events. There could be some difference of the rate of radiative events between data and the simulation. In the MC, about $15 \%$ of events contain a radiated photon. To evaluate the uncertainties due to this possible difference, we increase by $30 \%$ the rate of radiative events in the simulation. The variation of fitted parameters are shown in Tables 7.7 and 7.8 .

\begin{tabular}{|l|c|c|c|c|}
\hline corrections & $\delta\left(N_{s}\right)$ & $\delta\left(r_{0}\right)$ & $\delta\left(r_{V}\right)$ & $\delta\left(r_{2}\right)$ \\
\hline radiative events & +2 & -0.2 & -0.018 & -0.005 \\
\hline
\end{tabular}

Table 7.7: Variations of fitted parameters: $N_{s}, r_{0}\left(\right.$ in $\left.\mathrm{GeV}^{-1}\right), r_{2}, r_{V}$.

In the CLEO-c measurement of $D \rightarrow K^{-} e^{+} \nu_{e}$ [68] decays they have compared simulated radiative events using PHOTOS and KLOR [69] and observed that this simulates a change in the radiative branching fraction of up to $16 \%$ in the most extreme case. For $D_{s}^{+} \rightarrow \phi e^{+} \nu_{e}$ we expect smaller effects as the available phase space is reduced so we consider that the variation of $30 \%$ we have used overestimates the uncertainty from this source. 


\begin{tabular}{|l|c|c|c|c|c|}
\hline corrections & $\delta\left(N_{s}\right)$ & $\delta\left(r_{0}\right)$ & $\delta\left(r_{V}\right)$ & $\delta\left(r_{2}\right)$ & $\delta\left(\lambda_{A}\right)$ \\
\hline radiative events & +5 & -0.3 & -0.028 & +0.011 & +0.03 \\
\hline
\end{tabular}

Table 7.8: Variations of fitted parameters: $N_{s}, r_{0}$ (in $\mathrm{GeV}^{-1}$ ), $r_{2}, r_{V}$ and $\lambda_{A}$ (in $\left.\left(\mathrm{GeV} / \mathrm{c}^{2}\right)^{1 / 2}\right)$.

\subsection{S-wave parameterization}

The uncertainty due to the parameterization that we choose for the $S$-wave component, is mainly coming from the phase of the $f_{0}$ obtained in Equation 1.44. If we use a constant phase with value equal to 0 , the following variation is obtained on the parameter $r_{0}: \delta\left(r_{0}\right)=$ $+0.3 \mathrm{GeV}^{-1}$.

\section{7 $\quad$ Summary on systematic effects}

In Table 7.9 and 7.10, we give a summary of the different systematic contributions on the measurement of $r_{V}, r_{2}$ and $r_{0}$, and of $r_{V}, r_{2}, r_{0}$ and $\lambda_{A}$, respectively. The corresponding errors matrices are the following:

$$
\begin{gathered}
\left(\begin{array}{cccc}
129036 & -106.1 & -7.728 & 1.611 \\
-106.1 & 0.78 & 0.0116 & -0.0105 \\
-7.728 & 0.0116 & 0.002574 & 0.000744 \\
1.611 & -0.0105 & 0.000744 & 0.000862
\end{array}\right) \\
\left(\begin{array}{ccccc}
0.0082 & 1.4 & -0.028 & 7.10^{-5} & 0.00154 \\
1.4 & 135482 & -153.9 & -14.527 & 3.754 \\
-0.028 & -153.9 & 1.01 & 0.0433 & 0.0117 \\
7.10^{-5} & -14.527 & 0.0433 & 0.006223 & -0.0004 \\
0.00154 & 3.754 & 0.0117 & -0.0004 & 0.003852
\end{array}\right)
\end{gathered}
$$

We consider that the systematics on the form factors parameters are similar for the fit with or without the $S$-wave component. 


\begin{tabular}{lcccc}
\hline Source & error on $N_{s}$ & error on $r_{0}$ & error on $r_{V}$ & error on $r_{2}$ \\
\hline \hline Fisher variables for signal MC & 1 & 0 & 0.012 & 0.011 \\
smearing $D_{s}^{+}$angles & 10 & 0.1 & 0.001 & 0.005 \\
smearing missing energy & 13 & 0.1 & 0.001 & 0.004 \\
\hline total signal MC corrections & 16 & 0.1 & 0.012 & 0.013 \\
\hline \hline Fragmentation correction on $c \bar{c}$ events & 37 & 0.1 & 0.003 & 0 \\
Fisher variables for $B \bar{B}$ events & 230 & 0 & 0.033 & 0.002 \\
continuum bkg. & 198 & 0.5 & 0.018 & 0 \\
$\phi$ from fragmentation & 118 & 0 & 0.008 & 0.003 \\
$\phi$ from c-hadrons & 77 & 0 & 0.004 & 0.002 \\
$\phi$ from uds & 95 & 0 & 0.006 & 0.002 \\
\hline total background MC corrections & 350 & 0.5 & 0.039 & 0.005 \\
\hline \hline Monte-Carlo statistics & 81 & - & 0.022 & 0.017 \\
PID efficiencies & 3 & 0.6 & 0.001 & 0.019 \\
neutral correction & 1 & 0.1 & 0.009 & 0.002 \\
radiative events & 2 & 0.2 & 0.018 & 0.005 \\
$S-$ wave parameterization & - & 0.3 & - & - \\
\hline \hline Total & 360 & 0.9 & 0.051 & 0.030 \\
\hline \hline
\end{tabular}

Table 7.9: Systematic uncertainties on $N_{s}, r_{0}\left(\right.$ in $\left.\mathrm{GeV}^{-1}\right), r_{V}$ and $r_{2}$. The quoted numbers correspond to absolute errors. 


\begin{tabular}{lccccc}
\hline Source & error on $N_{s}$ & error on $r_{0}$ & error on $r_{V}$ & error on $r_{2}$ & error on $\lambda_{A}$ \\
\hline \hline Fisher variables for signal MC & 1 & 0.1 & 0.013 & 0.009 & 0 \\
smearing $D_{s}^{+}$angles & 10 & 0 & 0.001 & 0.005 & 0 \\
smearing missing energy & 5 & 0.1 & 0.009 & 0.013 & 0.02 \\
\hline total signal MC corrections & 11 & 0.1 & 0.016 & 0.017 & 0.02 \\
\hline \hline Fragmentation corr. on $c \bar{c}$ events & 38 & 0.1 & 0.007 & 0.004 & 0.01 \\
Fisher variables for $B \bar{B}$ events & 236 & 0.1 & 0.050 & 0.024 & 0.04 \\
continuum bkg. & 202 & 0.6 & 0.032 & 0.023 & 0.03 \\
$\phi$ from fragmentation & 120 & 0.1 & 0.019 & 0.013 & 0.03 \\
$\phi$ from c-hadrons & 80 & 0.1 & 0.002 & 0.013 & 0.02 \\
$\phi$ from uds & 100 & 0.1 & 0.007 & 0.024 & 0.03 \\
\hline total bckg MC corrections & 358 & 0.6 & 0.063 & 0.045 & 0.07 \\
\hline \hline Monte-Carlo statistics & 81 & - & 0.029 & 0.034 & 0.04 \\
PID efficiencies & 1 & 0.6 & 0.006 & 0.011 & 0.01 \\
neutral correction & 5 & 0.2 & 0.018 & 0.012 & 0.02 \\
radiative events & 5 & 0.3 & 0.028 & 0.011 & 0.03 \\
$S-$ wave parameterization & - & 0.3 & - & - & - \\
\hline \hline Total & 367 & 1 & 0.079 & 0.062 & 0.09 \\
\hline \hline
\end{tabular}

Table 7.10: Systematic uncertainties on $N_{s}, r_{0}, r_{V}, r_{2}$ and $\lambda_{A}$. For $r_{0}$ and $\lambda_{A}$ units are respectively $\mathrm{GeV}^{-1}$ and $\left(\mathrm{GeV} / \mathrm{c}^{2}\right)^{1 / 2}$. The quoted numbers correspond to absolute errors. 
We can see that the errors on $r_{0}, r_{V}$ and $r_{2}$ increase when the axial-vector pole mass is fitted. Especially, for the background MC corrections, the systematics on $r_{2}$ are really small when $\lambda_{A}$ is fixed but they become important when $\lambda_{A}$ is fitted. To investigate this effect, we take the example of the systematic uncertainty on the continuum background, and we try to understand the effects of the variation of this background on the kinematic variables. From Figure 4.41, we see that this background is almost constant as function of $\cos \theta_{V}$ and $\chi$, so we can restrain the study to the two dimensional space $\left(q^{2} \times \cos \theta_{\ell}\right)$. Figure 7.4 shows the difference between the reference background distribution and the background distribution when $10 \%$ of continuum background is added. The two plots show the same distribution with two different representations. Now, we compare this distribution with the ones resulting from the variations of the fitted parameters (which are used to evaluate the systematic uncertainties). We expect that these variations compensate the background changes.

For signal events, the difference between the kinematic distribution obtained with the nominal fitted values and the one obtained when $10 \%$ of continuum background is added are given on Figure 7.5 and 7.6, fixing or letting $\lambda_{A}$ to vary in the fit, respectively. These distributions are obtained with toy experiments. In both cases, the fit tries to compensate the shape of the background variation and it is more successful when there is one more degree of freedom. The fact that the variation of $r_{2}$ is zero when the axial-vector mass pole is fixed seems not to be a problem of the fit, which obtains the best minimization varying only $r_{V}$. From this study we show that the different parameters are correlated, and their behaviour, when a component such as the continuum background is changed, is not obvious because it generally takes place in a four dimensional space.
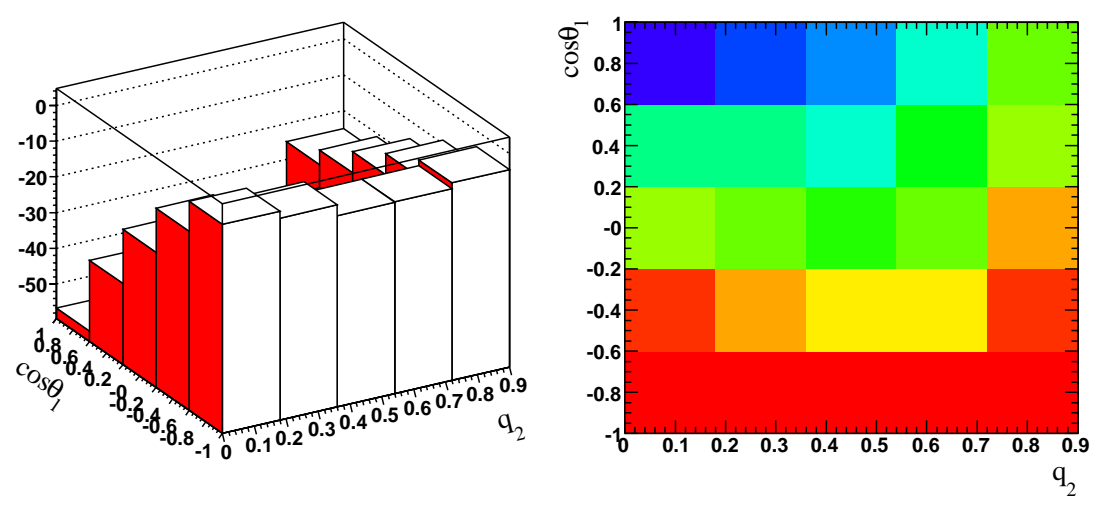

Figure 7.4: $q^{2}$ versus $\cos \theta_{\ell}$ distribution as obtained with background MC events. The plots show the difference between the background used in the nominal fit and the one obtained adding $10 \%$ of continuum background. 

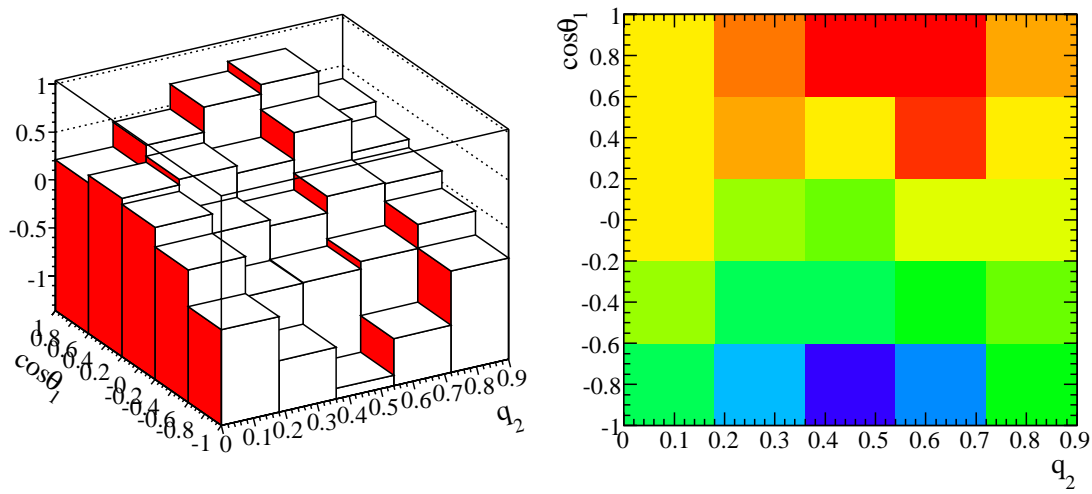

Figure 7.5: $q^{2}$ versus $\cos \theta_{\ell}$ distribution as obtained with toy experiments. The plots show the difference between the signal distribution using the parameters of the nominal fit and the one obtained with the parameters fitted when adding $10 \%$ of continuum background. Here the fit is done fixing the axial-vector pole mass.
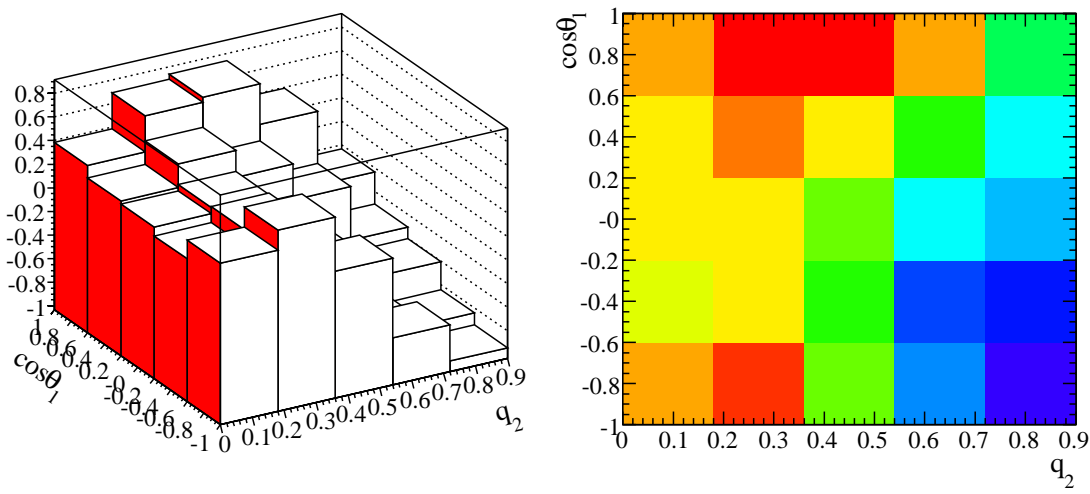

Figure 7.6: $q^{2}$ versus $\cos \theta_{\ell}$ distribution as obtained with toy experiments. The plots show the difference between the signal distribution using the parameters of the nominal fit and the one obtained with the parameters fitted when adding $10 \%$ of continuum background. Here, the axial-vector pole mass is a free paramter. 
To summarize the results obtained for the form factors parameters and the $S$-wave component, fitting $r_{2}, r_{V}$ and $r_{0}$, we obtain:

$$
\begin{gathered}
r_{V}=V(0) / A_{1}(0)=1.836 \pm 0.047 \pm 0.051, r_{2}=A_{2}(0) / A_{1}(0)=0.814 \pm 0.036 \pm 0.030 \\
\text { and } r_{0}=15.0 \pm 2.5 \pm 0.9 \mathrm{GeV}^{-1}
\end{gathered}
$$

Fitting also the axial-vector pole mass:

$$
\begin{gathered}
r_{V}=V(0) / A_{1}(0)=1.868 \pm 0.061 \pm 0.079, r_{2}=A_{2}(0) / A_{1}(0)=0.763 \pm 0.072 \pm 0.062 \\
m_{A}=\left(2.30_{-0.18}^{+0.24} \pm 0.21\right) \mathrm{GeV} / \mathrm{c}^{2}, \text { and } \mathrm{r}_{0}=(15.3 \pm 2.6 \pm 1) \mathrm{GeV}^{-1}
\end{gathered}
$$

We quote also the results obtained without considering the $S$-wave component, to compare with the previous experiments:

$r_{V}=V(0) / A_{1}(0)=1.799 \pm 0.046 \pm 0.051$ and $r_{2}=A_{2}(0) / A_{1}(0)=0.813 \pm 0.036 \pm 0.030$.

Fitting also the axial-vector mass pole:

$$
\begin{gathered}
r_{V}=V(0) / A_{1}(0)=1.831 \pm 0.060 \pm 0.079, r_{2}=A_{2}(0) / A_{1}(0)=0.758 \pm 0.070 \pm 0.062 \\
\text { and } m_{A}=\left(2.30_{-0.18}^{+0.24} \pm 0.21\right) \mathrm{GeV} / \mathrm{c}^{2}
\end{gathered}
$$




\section{Chapter 8}

\section{Decay rate measurement}

'Get to your places!' shouted the Queen in a voice of thunder, and people began running about in all directions, tumbling up against each other; however, they got settled down in a minute or two, and the game began. Alice thought she had never seen such a curious croquetground in all her life; it was all ridges and furrows; the balls were live hedgehogs, the mallets live flamingoes, and the soldiers had to double themselves up and to stand on their hands and feet, to make the arches.

In the following, we present a measurement of the production rate of $D_{s}^{+} \rightarrow K^{+} K^{-} e^{+} \nu_{e}$ events relative to $D_{s}^{+} \rightarrow K^{+} K^{-} \pi^{+}$, in a narrow range $\Delta m_{K K}$ around the $\phi$ mass peak, and we compare this value with a similar ratio obtained using simulated events. In this way, several systematic uncertainties cancel. We use as much as possible the same criteria to select events in the two decay channels. In simulated events, we restrict the signal to $K^{+} K^{-}$ coming from a $\phi$, while in data there could be also non resonant contribution, $D_{s}^{+} \rightarrow \bar{K}^{* 0} K^{+}$ and $D_{s}^{+} \rightarrow f_{0} \pi^{+}$decays. This is because with simulated events we are interested in measuring the analysis efficiency and it is legitimate to consider only the $D_{s}^{+}$decay into $\phi$ for which we have a complete control, whereas it is necessary to include all contributing channels in data to compare with the corresponding measurement done by CLEO-c, using similar selection criteria. 


\subsection{Branching fraction measurement}

To obtain the value for the $D_{s}^{+} \rightarrow K^{+} K^{-} e^{+} \nu_{e}$ decay branching fraction we consider the following ratio:

$$
\begin{aligned}
R_{D s}=\frac{B R\left(D_{s}^{+} \rightarrow K^{+} K^{-} e^{+} \nu_{e}\right)_{\text {data }}^{\Delta m_{1}}}{B R\left(D_{s}^{+} \rightarrow K^{+} K^{-} \pi^{+}\right)_{\text {data }}^{\Delta m_{2}}} & =\frac{N\left(D_{s}^{+} \rightarrow K^{+} K^{-} e^{+} \nu_{e}\right)_{\text {data }}^{\Delta m_{1}}}{\epsilon\left(D_{s}^{+} \rightarrow K^{+} K^{-} e^{+} \nu_{e}\right)_{\text {data }}^{\Delta m_{1}}} \\
& \times \frac{\epsilon\left(D_{s}^{+} \rightarrow K^{+} K^{-} \pi^{+}\right)_{\text {data }}^{\Delta m_{2}}}{N\left(D_{s}^{+} \rightarrow K^{+} K^{-} \pi^{+}\right)_{\text {data }}^{\Delta m_{2}}}
\end{aligned}
$$

The indices $\Delta m_{1}$ and $\Delta m_{2}$ refer to the considered $K^{+} K^{-}$mass range for $D_{s}^{+} \rightarrow K^{+} K^{-} e^{+} \nu_{e}$ and $D_{s}^{+} \rightarrow K^{+} K^{-} \pi^{+}$events respectively. They are different because for the $D_{s}^{+} \rightarrow K^{+} K^{-} \pi^{+}$ events we use the same mass interval as CLEO[70] $\left(1.0095<m_{K K}<1.0295 \mathrm{GeV} / \mathrm{c}^{2}\right)$, but for $D_{s}^{+} \rightarrow K^{+} K^{-} e^{+} \nu_{e}$ events we keep the range used for the form factors analysis $\left(1.01<m_{K K}<1.03 \mathrm{GeV} / \mathrm{c}^{2}\right)$. The quantities $N$ correspond to the measured number of events in the two decay channels and the corresponding efficiencies, $\epsilon$, are evaluated from the simulation and corrected for possible differences between data and simulated events:

$$
\epsilon\left(D_{s}^{+} \rightarrow K^{+} K^{-} e^{+} \nu_{e}\right)_{\text {data }}^{\Delta m_{1}}=\epsilon\left(D_{s}^{+} \rightarrow \phi e^{+} \nu_{e}\right)_{M C}^{\Delta m_{1}} \times \frac{\epsilon\left(D_{s}^{+} \rightarrow K^{+} K^{-} e^{+} \nu_{e}\right)_{\text {data }}^{\Delta m_{1}}}{\epsilon\left(D_{s}^{+} \rightarrow \phi e^{+} \nu_{e}\right)_{M C}^{\Delta m_{1}}}
$$

Efficiencies measured using the simulation are obtained by comparing the number of reconstructed and generated events in the considered channel:

$$
\epsilon\left(D_{s}^{+} \rightarrow \phi e^{+} \nu_{e}\right)_{M C}^{\Delta m_{1}}=\frac{N\left(D_{s}^{+} \rightarrow \phi e^{+} \nu_{e}\right)_{M C r e c}^{\Delta m_{1}}}{N\left(D_{s}^{+} \rightarrow \phi e^{+} \nu_{e}\right)_{M C g e n}^{\Delta m_{1}}}
$$

The number of generated events analyzed in the simulation depends on the statistics of continuum $e^{+} e^{-} \rightarrow c \bar{c}$, on the probability that a charm quark transforms into a $D_{s}$ during fragmentation $\left(\mathcal{P}\left(c \rightarrow D_{s}^{+}\right)\right)$and on the value for the decay branching fraction of the $D_{s}$ meson into the studied channel:

$$
N\left(D_{s}^{+} \rightarrow \phi e^{+} \nu_{e}\right)_{M C g e n .}=2 N(c \bar{c})_{\phi e \nu} \mathcal{P}\left(c \rightarrow D_{s}^{+}\right) B R\left(D_{s}^{+} \rightarrow \phi e^{+} \nu_{e}\right)_{M C} B R\left(\phi \rightarrow K^{+} K^{-}\right)_{M C}
$$


The corresponding number of events in the $K^{+} K^{-}$range is:

$$
N\left(D_{s}^{+} \rightarrow \phi e^{+} \nu_{e}\right)_{M C g e n .}^{\Delta m_{1}}=N\left(D_{s}^{+} \rightarrow \phi e^{+} \nu_{e}\right)_{M C g e n .} \times F\left(D_{s}^{+} \rightarrow \phi e^{+} \nu_{e}\right)
$$

where $F\left(D_{s}^{+} \rightarrow \phi e^{+} \nu_{e}\right)$ is the ratio between the number of $D_{s}^{+} \rightarrow \phi \nu_{e}$ generated in $\Delta m_{1}$ and the number of $D_{s}^{+} \rightarrow \phi e^{+} \nu_{e}$ generated over the whole $m_{K K}$ spectrum:

$$
F\left(D_{s}^{+} \rightarrow \phi e^{+} \nu_{e}\right)=\frac{N\left(D_{s}^{+} \rightarrow \phi e^{+} \nu_{e}\right)_{M C g e n}^{\Delta m_{1}}}{N\left(D_{s}^{+} \rightarrow \phi e^{+} \nu_{e}\right)_{M C g e n}^{\text {total }}}
$$

This ratio is evaluated from the simulation.

Similar expressions are used for the $D_{s}^{+} \rightarrow \phi \pi^{+}$channel, replacing $\Delta m_{1}$ by $\Delta m_{2}$.

The numbers of reconstructed events in data are proportional to the analyzed integrated luminosities $\mathcal{L}($ data $)$ for the two channels, respectively. Re-ordering the different terms and eliminating a few parameters as $\mathcal{P}\left(c \rightarrow D_{s}^{+}\right)$and $B R\left(\phi \rightarrow K^{+} K^{-}\right)_{M C}$, Equation (8.1) becomes:

$$
\begin{aligned}
R_{D s}=\frac{B R\left(D_{s}^{+} \rightarrow K^{+} K^{-} e^{+} \nu_{e}\right)_{\text {data }}^{\Delta m_{1}}}{B R\left(D_{s}^{+} \rightarrow K^{+} K^{-} \pi^{+}\right)_{\text {data }}^{\Delta m_{2}}} & =\frac{B R\left(D_{s}^{+} \rightarrow \phi e^{+} \nu_{e}\right)_{M C}^{\Delta m_{1}}}{B R\left(D_{s}^{+} \rightarrow \phi \pi^{+}\right)_{M C}^{\Delta m_{2}}} \\
& \times \frac{N(c \bar{c})_{\phi e \nu}}{N(c \bar{c})_{\phi \pi}} \times \frac{\mathcal{L}(\text { data })_{K K \pi}}{\mathcal{L}(\text { data })_{K K e \nu}} \times \frac{F\left(D_{s}^{+} \rightarrow \phi e^{+} \nu_{e}\right)}{F\left(D_{s}^{+} \rightarrow \phi \pi^{+}\right)} \\
& \times \frac{N\left(D_{s}^{+} \rightarrow K^{+} K^{-} e^{+} \nu_{e}\right)_{\text {data }}^{\Delta m_{1}}}{N\left(D_{s}^{+} \rightarrow \phi e^{+} \nu_{e}\right)_{M C}^{\Delta m_{1}}} \times \frac{N\left(D_{s}^{+} \rightarrow \phi \pi^{+}\right)_{M C}^{\Delta m_{2}}}{N\left(D_{s}^{+} \rightarrow K^{+} K^{-} \pi^{+}\right)_{\text {data }}^{\Delta m_{2}}} \\
& \times \frac{\epsilon\left(D_{s}^{+} \rightarrow \phi e^{+} \nu_{e}\right)_{M C}}{\epsilon\left(D_{s}^{+} \rightarrow K^{+} K^{-} e^{+} \nu_{e}\right)_{\text {data }}} \times \frac{\epsilon\left(D_{s}^{+} \rightarrow K^{+} K^{-} \pi^{+}\right)_{\text {data }}}{\epsilon\left(D_{s}^{+} \rightarrow \phi \pi^{+}\right)_{M C}}
\end{aligned}
$$

The first line, in this expression, corresponds to the ratio between the branching fractions for the two channels used in the simulation:

$$
\frac{B R\left(D_{s}^{+} \rightarrow \phi e^{+} \nu_{e}\right)_{M C}}{B R\left(D_{s}^{+} \rightarrow \phi \pi^{+}\right)_{M C}}=\frac{0.0242}{0.044}
$$

The second line corresponds to the ratio between the number of $c \bar{c}$ simulated events and between the integrated luminosities analyzed for the two channels:

$$
\frac{N(c \bar{c})_{\phi e \nu}}{N(c \bar{c})_{\phi \pi}} \times \frac{\mathcal{L}(\text { data })_{K^{+} K^{-\pi}}}{\mathcal{L}(\text { data })_{K^{+} K^{-} e \nu}}=\frac{480.78 \times 10^{6}}{150.58 \times 10^{6}} \times \frac{100.314 f b^{-1}}{214.204 f b^{-1}}
$$

The third line corresponds to the ratios between measured numbers of signal events in data and in the simulation for the two channels. The last line corresponds to the efficiencies for 
data and simulation. In this double ratio most of differences between data and simulation are expected to cancel.

The CLEO collaboration has determined a value for the branching fraction of the decay $D_{s}^{+} \rightarrow K^{+} K^{-} \pi^{+}[70]$ using a cut on the invariant $K^{+} K^{-}$mass : $1.0095<m_{K^{+} K^{-}}<$ $1.0295 \mathrm{GeV} / \mathrm{c}^{2}$ ( which corresponds to $\Delta m_{2}$ ). They obtain:

$$
B R\left(D_{s}^{+} \rightarrow K^{+} K^{-} \pi^{+}\right)_{\text {data }}^{\Delta m_{2}}=(1.99 \pm 0.10 \pm 0.05) \%
$$

\subsection{Selection of $D_{s}^{+} \rightarrow K^{+} K^{-} \pi^{+}$events}

The same lists for charged and neutral particles as used in semileptonic events are selected. Events are also analyzed in the same way, separating reconstructed particles in two hemispheres defined from the thrust axis. In each hemisphere a $D_{s}$ candidate is reconstructed by combining two kaons of opposite sign with a pion. These tracks are required to form a vertex and the two-kaons invariant mass is required to be in the range [1.0095, 1.0295] GeV/c $\mathrm{c}^{2}$.

In addition, the following selection criteria are applied:

- the direction of the thrust axis must verify $\left|\cos \theta_{\text {thrust }}\right|<0.6$;

- the $D_{s}$ candidate momentum in the center-of-mass is required to exceed $2.5 \mathrm{GeV} / \mathrm{c}$ to remove contribution from $B \bar{B}$ events;

- at least one spectator particle accompanying the $D_{s}$ candidate in its hemisphere;

- the $K K \pi$ mass is selected in the range between 1.953 and $1.983 \mathrm{GeV} / \mathrm{c}^{2}$ for the data and between 1.9539 and $1.9839 \mathrm{GeV} / \mathrm{c}^{2}$ for the simulation;

- the $\chi^{2}$ probability for the $D_{s}^{+}$vertex is required to be greater than 0.01 ;

- the same cut on the Fisher variable $F_{b b}$ against $B \bar{B}$ events as used in the $D_{s}^{+} \rightarrow$ $K^{+} K^{-} e^{+} \nu_{e}$ event selection;

- a cut on the second Fox-Wolfram moment $R_{2}>0.2$, this is a selection cut of the skim used to select semileptonic events;

The $D_{s}$ mass distribution is shown in Figure 8.1. As it can be seen from the MC, there is a small component of signal composed of non-resonant events, $D_{s}^{+} \rightarrow f_{0} \pi^{+}$with $f_{0} \rightarrow K^{+} K^{-}$ and $D_{s}^{+} \rightarrow \bar{K}^{* 0} K^{+}$with $\bar{K}^{* 0} \rightarrow \pi^{+} K^{-}$. We choose not to cut on the cosine between the pion and one of the kaons in order to have a similar efficiency for all components. Moreover 
the contribution from non-resonant events is kept as we use the $D_{s}^{+} \rightarrow K^{+} K^{-} \pi^{+}$branching ratio measurement, obtained in the same conditions, to extract the semileptonic decay rate. We observe that the $D_{s}$ mass distribution in the simulation has a small shift as compared to data. The estimated mean and width are determined from a Gaussian fit to the mass peak. We find: $\mu_{D s}^{\text {data }}=1.9677 \mathrm{GeV} / \mathrm{c}^{2}, \sigma_{D_{s}}^{\text {data }}=5.43 \mathrm{MeV} / \mathrm{c}^{2}$ and $\mu_{D s}^{M C}=1.9686 \mathrm{GeV} / \mathrm{c}^{2}$, $\sigma_{D_{s}}^{M C}=5.30 \mathrm{MeV} / \mathrm{c}^{2}$. To correct for this difference, we shift the $D_{s}$ mass cuts by $0.9 \mathrm{MeV} / \mathrm{c}^{2}$ for the simulation.

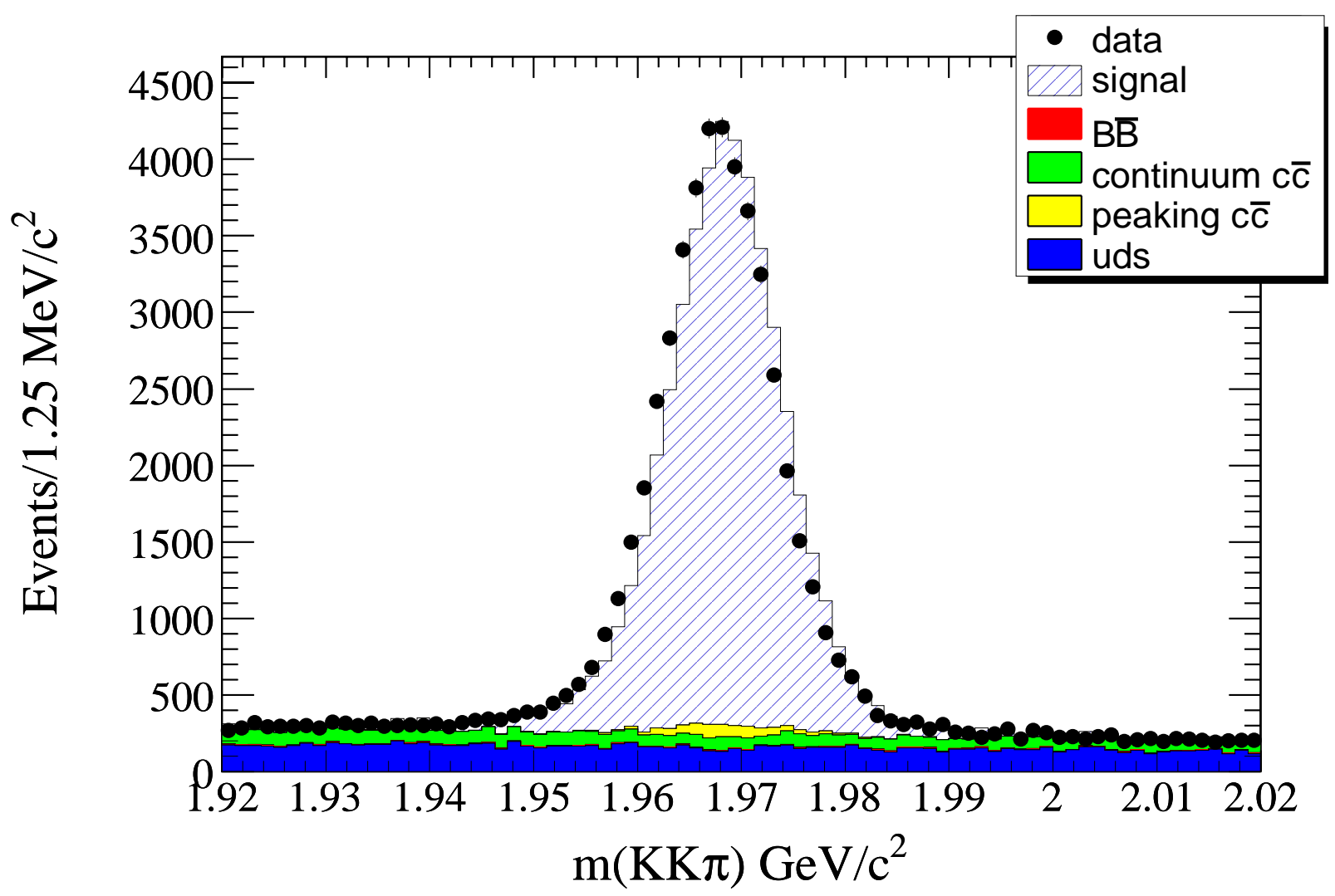

Figure 8.1: $K^{+} K^{-} \pi^{+}$mass distribution for real events and simulation.

To measure the number of $D_{s}^{+}$in data, the continuum background is subtracted using the sidebands in the same way as explained in Section 4.1 and the difference in the c quark fragmentation between real and simulated events is corrected by applying weights to the simulated events.

In simulated events it is required that the two kaons originate from a $\phi$ meson decay because we use the value of the corresponding branching fraction in the simulation. 
The numbers of $D_{s}^{+}$signal events obtained in data and simulation are the following:

$$
\frac{N\left(D_{s}^{+} \rightarrow \phi \pi^{+}\right)_{M C}^{\Delta m_{2}}}{N\left(D_{s}^{+} \rightarrow K^{+} K^{-} \pi^{+}\right)_{\text {data }}^{\Delta m_{2}}}=\frac{52994 \pm 230}{43070 \pm 232}=1.2304 \pm 0.0085
$$

The systematic uncertainty that will remain on this ratio corresponds to the difference between the pion reconstruction in data and in the simulation.

From the simulation, we obtain $F\left(D_{s}^{+} \rightarrow \phi \pi^{+}\right)=\frac{N\left(D_{s}^{+} \rightarrow \phi \pi^{+}\right)_{M C g e n}^{\Delta m_{2}}}{N\left(D_{s}^{+} \rightarrow \phi \pi^{+}\right)_{M C g e n}^{t o t a l}}=87.03 \pm 0.16 \%$.

\subsection{Selection of $D_{s}^{+} \rightarrow K^{+} K^{-} e^{+} \nu_{e}$ events}

The selection criteria are the following:

- criteria similar as those already applied on $K^{+} K^{-} \pi$ candidates: requirements on the thrust axis direction, at least one spectator particle accompanying the $D_{s}^{+}$candidate, cut on the $K^{+} K^{-}$mass system and on the Fisher variable $F_{b b}$;

- a cut on the Fisher variable $F_{c c}$ used against background charm events;

- the mass constrained fit has converged and the $\chi^{2}$ probability of the fit is larger than 0.01 ;

- the $\chi^{2}$ probability for the two kaons and lepton vertex is required to be greater than 0.01 ;

- the lepton momentum in the center of mass is required to be greater than $0.5 \mathrm{GeV} / \mathrm{c}^{2}$;

- the rejection of converted photons.

The same corrections on simulated signal and background events are applied as in the form factors measurement. The number of signal events in data is estimated by the fit described in chapter 5 , and performed in the $K^{+} K^{-}$mass range $1.01<m_{K K}<1.03 \mathrm{GeV} / \mathrm{c}^{2}$. The parameters $N_{s}, r_{2}, r_{V}, r_{0}$ and $\lambda_{A}$ are left free to vary in this fit.

To evaluate the number of signal events in the simulation, we replace the generic $c \bar{c} \mathrm{MC}$ by the signal MC generated with the simple pole model. This last sample is normalized to the same generated number of signal events as in the generic MC sample. As the skim (see section 3.2.1) is applied on the data but not on the specific MC, we apply the cuts defined in the skim at the reconstruction level. The number of signal events obtained in 
the simulation is $N\left(D_{s}^{+} \rightarrow \phi e^{+} \nu_{e}\right)_{M C}^{\Delta m_{1}}=46465$, with the form factors paramters $r_{2}=0.7$, $r_{V}=1.5, m_{A}=2.5 \mathrm{GeV} / \mathrm{c}^{2}$. If we reweight the $\mathrm{MC}$ using the fitted value of the parameters $\left(r_{2}=0.763, r_{V}=1.868, m_{A}=2.30 \mathrm{GeV} / \mathrm{c}^{2}\right)$, we find a variation of $(-1.20) \%$ on the number of signal events. There is an uncertainty on this variation originating from the uncertainties of the fitted parameters. As the fitted parameters values are needed also in the final part of the calculation to obtain the value of $A_{1}(0)$, we treat the corresponding errors at the end, taking into account correlations. We obtain $N\left(D_{s}^{+} \rightarrow \phi e^{+} \nu_{e}\right)_{M C}^{\Delta m_{1}}=45907 \pm 214$. The numbers of $D_{s}^{+}$signal events obtained in data and simulation are the following:

$$
\frac{N\left(D_{s}^{+} \rightarrow K^{+} K^{-} e^{+} \nu_{e}\right)_{\text {data }}^{\Delta m_{1}}}{N\left(D_{s}^{+} \rightarrow \phi e^{+} \nu_{e}\right)_{M C}^{\Delta m_{1}}}=\frac{25152 \pm 177}{45907 \pm 214}=0.5479 \pm 0.0046
$$

From the signal MC, we obtain $F\left(D_{s}^{+} \rightarrow \phi e^{+} \nu\right)=86.89 \pm 0.29 \%$.

\subsection{Measured value of $R_{D s}$ and statistical uncertainty}

Combining the different measured quantities, one gets:

$$
\begin{aligned}
R_{D s}=\frac{B R\left(D_{s}^{+} \rightarrow K^{+} K^{-} e^{+} \nu_{e}\right)_{\text {data }}^{\Delta m_{1}}}{B R\left(D_{s}^{+} \rightarrow K^{+} K^{-} \pi^{+}\right)_{\text {data }}^{\Delta m_{2}}} & =\frac{0.0242}{0.044} \\
& \times \frac{480.78}{150.58} \times \frac{100.314}{214.204} \times \frac{86.89 \pm 0.29}{87.03 \pm 0.16} \\
& \times(0.5479 \pm 0.0048) \times(1.2304 \pm 0.0085) \\
& =0.5535 \pm 0.0064
\end{aligned}
$$

\subsection{Systematic uncertainties on $R_{D s}$}

Systematic uncertainties on $R_{D s}$ originate from quantities which are used to select $D_{s}^{+} \rightarrow$ $K^{+} K^{-} e^{+} \nu_{e}$ and which are different from those used in the selection of $D_{s}^{+} \rightarrow K^{+} K^{-} \pi^{+}$(cut on the Fisher discriminant against $c \bar{c}$ events and mass constrained fit), from the differences in the PID requirements for the two channels and simulation of radiative events. The systematic errors on the evaluation of the number of semileptonic events in data are also taken into account. 


\subsubsection{Cut on the Fisher discriminant variables}

The stability of the fraction of events selected in data and in the simulation versus the cut applied on the Fisher discriminant variable designed to reduce the charm background has been verified.

In Figure 4.43 the distribution of the Fisher variable used against the charm background is compared with expectation from the simulation. Background and signal events from simulation are normalized to the same data luminosity as analyzed in real events.

\begin{tabular}{|c|c|c|c|}
\hline & $F_{c c}>-0.3$ & $F_{c c}>0$ & $F_{c c}>0.3$ \\
\hline DATA/MC & 0.5444 & 0.5465 & 0.5541 \\
\hline signal/backg. & 2.6 & 3.8 & 6.4 \\
\hline
\end{tabular}

Table 8.1: Ratio of the numbers of $D_{s}^{+} \rightarrow K^{+} K^{-} e^{+} \nu_{e}$ signal events in data and in simulated events for different cuts on the Fisher variable $F_{c c}$.

We quote, as systematics, the difference between the measurement using a cut $>-0.3$ and the one using a cut $>0.3$, which are the two extreme values. This gives a relative error on $R_{D s}$ of $1.77 \%$.

The same study has been done for the Fisher variable against the $B \bar{B}$ background, the results are given in Table 8.2. Here, we vary simultaneously the cut value for $D_{s}^{+} \rightarrow$ $K^{+} K^{-} e^{+} \nu_{e}$ and $D_{s}^{+} \rightarrow K^{+} K^{-} \pi^{+}$and we quote the $R_{D s}$ value obtained with this variation. We quote as systematic uncertainties, the difference between the measurement using a cut $>-0.3$ and the one using a cut $>0$. This gives a relative error on $R_{D s}$ of $0.58 \%$.

\begin{tabular}{|c|c|c|c|}
\hline & $F_{b b}>-0.3$ & $F_{b b}>0$ & $F_{b b}>0.3$ \\
\hline$R_{D s}$ & 0.5489 & 0.5521 & 0.5513 \\
\hline signal/backg. & 2.8 & 3.8 & 4.4 \\
\hline
\end{tabular}

Table 8.2: Value of $R_{D s}$ obtained for different values of the cut on the Fisher variable $F_{b b}$. The second line gives the signal/background ratio of $D_{s}^{+} \rightarrow K^{+} K^{-} e^{+} \nu_{e}$ events.

\subsubsection{Number of events $D_{s}^{+} \rightarrow K^{+} K^{-} e^{+} \nu_{e}$}

As we use the fit to evaluate the number of $D_{s}^{+} \rightarrow K^{+} K^{-} e^{+} \nu_{e}$ events, we can use the systematic uncertainty computed in Section 7: $\sigma(N)=367$. This error includes the error on background estimation, correction on simulated signal events and difference on the electron 
efficiency between real and simulated events. Using this variation on the number of $D_{s}^{+} \rightarrow$ $K^{+} K^{-} e^{+} \nu_{e}$ events in data, we find a relative error of $1.46 \%$ on the determination of $R_{D s}$.

\subsubsection{Mass constrained fit probability}

The difference in the efficiency between data and simulated events for the cut on the mass constrained fit probability has been evalutated for $D^{0} \rightarrow K^{-} e^{+} \nu$. An excess of $0.6 \%$ has been found for the MC efficiency. Using this number, we obtain a variation on $R_{D s}$ of $0.61 \%$.

\subsubsection{Particle identification}

The differences between data and MC in the PID efficiency that could lead to systematic uncertainties are coming from the electron and the pion, as differences in kaon efficiencies are expected to cancel in the ratio. We evaluate this uncertainty turning off the PID correction in both channels. This leads to a relative variation of $0.74 \%$ on $R_{D s}$.

\subsubsection{Radiative events}

As for the form factors measurement, the simulation of radiative events can be a source of systematic uncertainty if the rate of such events is different in data and simulation. If the efficiencies of radiative and non radiative events are different, a variation of the rate can lead to a variation of the number of reconstructed $D_{s}^{+} \rightarrow K^{+} K^{-} e^{+} \nu_{e}$ events. Using signal MC events, the following efficiencies have been measured: $\epsilon_{\text {rad }}=(4.70 \pm 0.03) \%$ and $\epsilon_{\text {nonrad. }}=(4.72 \pm 0.01) \%$. We do not see any significant difference between the two efficiency so the systematic uncertainty coming from simulation of radiative events is negligeable.

\subsubsection{Signal events evaluation}

We evaluate the number of simulated signal $D_{s}^{+} \rightarrow K^{+} K^{-} e^{+} \nu_{e}$ events and $D_{s}^{+} \rightarrow K^{+} K^{-} \pi^{+}$ events using corrections depending on the $D_{s}$ momentum, on the spectator system momentum and on the event shape variables. These corrections are expected to induce different variations on the number of events in $D_{s}^{+} \rightarrow K^{+} K^{-} e^{+} \nu_{e}$ and $D_{s}^{+} \rightarrow K^{+} K^{-} \pi^{+}$events. This is because the weight depending on the $D_{s}^{+}$momentum, used in these corrections, is greater than 1 for $D_{s}^{+}$with high momenta and $D_{s}^{+}$decaying semileptonicaly have on average more energy than $D_{s}^{+}$decaying into $\phi \pi$ (see Section 4.1).

If we do not apply these corrections, we obtain a relative variation on $R_{D s}$ of $6.9 \%$. 
Nevertheless, these corrections have been defined with a good accuracy and to evaluate the corresponding uncertainty we use an additional weight as function of $F_{b b}$ in both channels (as done for the systematic uncertainties on the form factors parameters). Doing that, we obtain a relative variation of $0.2 \%$ on $R_{D s}$.

\subsubsection{Summary on systematic errors}

In Table 8.3 have been summarized the values of systematic uncertainties contributing to the determination of $R_{D s}$.

\begin{tabular}{|c|c|}
\hline source & relative variation \\
\hline cut on Fisher variable $c$-evts & $1.77 \%$ \\
\hline cut on Fisher variable $b$-evts & $0.58 \%$ \\
\hline$D_{s}^{+} \rightarrow K^{+} K^{-} e^{+} \nu_{e}$ number of data events & $1.46 \%$ \\
\hline cut on mass constrained fit probability & $0.61 \%$ \\
\hline PID efficiency & $0.74 \%$ \\
\hline signal event evaluation & $0.2 \%$ \\
\hline total & $2.56 \%$ \\
\hline
\end{tabular}

Table 8.3: Summary of systematic uncertainties on the measurement of the relative decay rate.

\subsection{Decay rate measurement and value of the hadronic form factor at $q^{2}=0$}

The measured relative decay rate is:

$$
R_{D s}=\frac{B R\left(D_{s}^{+} \rightarrow K^{+} K^{-} e^{+} \nu_{e}\right)^{\Delta m_{1}}}{B R\left(D_{s}^{+} \rightarrow K^{+} K^{-} \pi^{+}\right)^{\Delta m_{2}}}=0.5535 \pm 0.0064 \pm 0.0142
$$

Using the CLEO value for the branching fraction $D_{s}^{+} \rightarrow K^{+} K^{-} \pi^{+}$within the selected $\Delta m_{K K}$ interval of $(1.99 \pm 0.10 \pm 0.05) \%$, it gives:

$$
B R\left(D_{s}^{+} \rightarrow K^{+} K^{-} e^{+} \nu_{e}\right)^{\Delta m_{1}}=(1.101 \pm 0.013 \pm 0.028 \pm 0.061) \times 10^{-2}
$$

where the last quoted uncertainty comes from the accuracy on $B R\left(D_{s}^{+} \rightarrow K^{+} K^{-} \pi^{+}\right)$. We remind that $\Delta m_{1}=[1.01,1.03] \mathrm{GeV} / \mathrm{c}^{2}$ in this equation. In [70], they measure $B R\left(D_{s}^{+} \rightarrow\right.$ $K^{+} K^{-} \pi^{+}$) for different mass intervals. We choose as reference the one which is more similar 
to the range used for the semileptonic channel, and we check the stability of $B R\left(D_{s}^{+} \rightarrow\right.$ $\left.K^{+} K^{-} e^{+} \nu_{e}\right)^{\Delta m_{1}}$ using the other CLEO measurements. The obtained values are summarized in Table 8.4, and are compatible within errors.

\begin{tabular}{|c|c|}
\hline$\Delta m_{K K}\left(\mathrm{MeV} / \mathrm{c}^{2}\right)$ & $B R\left(D_{s}^{+} \rightarrow K^{+} K^{-} e^{+} \nu_{e}\right)^{\Delta m_{1}}\left(\times 10^{-2}\right)$ \\
\hline 10 & $1.117 \pm 0.013 \pm 0.029 \pm 0.066$ \\
20 & $1.101 \pm 0.013 \pm 0.028 \pm 0.061$ \\
30 & $1.099 \pm 0.013 \pm 0.028 \pm 0.056$ \\
40 & $1.092 \pm 0.013 \pm 0.028 \pm 0.063$ \\
\hline
\end{tabular}

Table 8.4: Value of $B R\left(D_{s}^{+} \rightarrow K^{+} K^{-} e^{+} \nu_{e}\right)^{\Delta m_{1}}$ obtained for different $\Delta m_{K K}$ intervals centered on the $\phi$ mass resonance.

To obtain the branching fraction for the decay channel $D_{s}^{+} \rightarrow \phi e^{+} \nu_{e}$ the previous result has to be corrected for the following effects:

- there are $D_{s}^{+} \rightarrow \phi e^{+} \nu_{e}$ events with $m_{K K}$ outside the selected interval. To evaluate the corresponding fraction, we use a non-resonant $\mathrm{MC} D_{s}^{+} \rightarrow K^{+} K^{-} e^{+} \nu_{e}$ that we weight with a $\mathrm{P}$ wave, using the fitted value of $N_{s}, r_{2}, r_{V}$, and $m_{A}$. We do not use the signal $D_{s}^{+} \rightarrow \phi e^{+} \nu_{e}$ simulation because the mass distribution is cut above $1.08 \mathrm{GeV} / \mathrm{c}^{2}$ (see section 4.3.5). We obtain the following fraction:

$$
R_{\Delta m_{1}}=\frac{B R\left(D_{s}^{+} \rightarrow \phi e^{+} \nu_{e}\right)^{\Delta m_{1}}}{B R\left(D_{s}^{+} \rightarrow \phi e^{+} \nu_{e}\right)}=(86.37 \pm 1.22) \%
$$

The uncertainty on this ratio has been evaluated taking into account uncertainties on the $\phi$ meson mass distribution (essentialy coming from the Blatt-Weisskopf parameter, taken to be $r=3 \pm 2$ ) and on the fitted parameters;

- $D_{s}^{+} \rightarrow f_{0} e^{+} \nu_{e}$ events. Within the selected $m_{K K}$ interval, the measured fraction corresponding to the S-wave amounts to:

$$
R_{f_{0}}=\frac{N\left(D_{s}^{+} \rightarrow f_{0} e^{+} \nu_{e}\right)^{\Delta m_{1}}, f_{0} \rightarrow K^{+} K^{-}}{N\left(D_{s}^{+} \rightarrow K^{+} K^{-} e^{+} \nu_{e}\right)^{\Delta m_{1}}}=\left(0.23_{-0.08}^{+0.12}\right) 10^{-2} .
$$

The branching fraction for the decay $D_{s}^{+} \rightarrow \phi e^{+} \nu_{e}$ is then:

$$
\begin{array}{r}
B R\left(D_{s}^{+} \rightarrow \phi e^{+} \nu_{e}\right)=B R\left(D_{s}^{+} \rightarrow K+K-e^{+} \nu_{e}\right)^{\Delta m_{1}} \frac{1-R_{f_{0}}}{R_{\Delta m_{1}}} \frac{1}{B R\left(\phi \rightarrow K^{+} K^{-}\right)} \\
B R\left(D_{s}^{+} \rightarrow \phi e^{+} \nu_{e}\right)=(2.585 \pm 0.031 \pm 0.075 \pm 0.147) \times 10^{-2}
\end{array}
$$


To have the corresponding value of $A_{1}(0)$, we have to integrate Equation (1.25) over $q^{2}$, $\cos \left(\theta_{V}\right), \cos \left(\theta_{e}\right)$ and $\chi$. Integrating over the three angles, we obtain:

$$
d \Gamma=\frac{2 G_{F}^{2}\left|V_{c s}\right|^{2}}{3(4 \pi)^{3} m_{D}^{2}} p_{K K} * q^{2}\left(\left|H_{+}\left(q^{2}\right)\right|^{2}+\left|H_{-}\left(q^{2}\right)\right|^{2}+\left|H_{0}\left(q^{2}\right)\right|^{2}\right) d q^{2}
$$

The integration over $q^{2}$ gives:

$$
\Gamma=\frac{\hbar B R\left(D_{s}^{+} \rightarrow \phi e^{+} \nu_{e}\right)}{\tau_{D_{s}}}=\frac{2 G_{F}^{2}\left|V_{c s}\right|^{2}}{3(4 \pi)^{3} m_{D}^{2}}\left|A_{1}(0)\right|^{2} \mathcal{I}
$$

with

$$
\mathcal{I}=\frac{1}{\left|A_{1}(0)\right|^{2}} \int_{0}^{q_{\max }^{2}} p_{K K} * q^{2}\left(\left|H_{+}\left(q^{2}\right)\right|^{2}+\left|H_{-}\left(q^{2}\right)\right|^{2}+\left|H_{0}\left(q^{2}\right)\right|^{2}\right) d q^{2} .
$$

Using Equation 8.17, and calculating $\mathcal{I}$ numerically, we obtain the following value:

$$
A_{1}(0)=0.605 \pm 0.012 \pm 0.018 \pm 0.018
$$

In this last evaluation we have used the value $\tau_{D_{s}}=(500 \pm 7) \times 10^{-15} s$ for the $D_{s}$ lifetime and $\left|V_{c s}\right|=0.9729 \pm 0.0003$. The three errors on $A_{1}(0)$ correspond respectively to the statistical error, the systematic errors, and errors from external inputs as branching ratios, $\tau_{D_{s}}$ and $\left|V_{c s}\right|$. Errors of the form factors parameters $r_{2}, r_{V}$ and $m_{A}$ affect the measurement of $A_{1}(0)$ by the intermediate of $\mathcal{I}$ and of the number of signal $D_{s}^{+} \rightarrow \phi e^{+} \nu_{e}$ events in the simulation. The correlations between these two quantities are taken into account, and we evaluate separately contributions of the statistical and systematic errors on $\sigma\left(A_{1}(0)\right)$. We obtain $\sigma_{\text {stat }}\left(A_{1}(0)\right)=0.010$ and $\sigma_{\text {syst }}\left(A_{1}(0)\right)=0.015$. These uncertainties are included in the result quoted in Equation (8.19). 


\title{
Chapter 9
}

\section{Physics interpretations}

\author{
At this the whole pack rose up into the air, and came \\ flying down upon her: she gave a little scream, half of \\ fright and half of anger, and tried to beatthem off, and \\ found herself lying on the bank, with her head in the lap \\ of her sister, who was gently brushing away some dead \\ leaves that had fluttered down from the tree upon her \\ face. \\ 'Wake up, Alice dear!', said her sister; 'Why, what a \\ long sleep you've had!'
}

In this chapter, we give comparisons between our results and other experimental measurements, and theoretical calculations. Based on the formalism given in [27], we also try to interpret in a very simple way these results in the framework of HQET, taking into account the first order corrections.

\subsection{Form factors parameterization}

Using Eq.(8.17) and the fitted values of the form factor parameters for the $D_{s}^{+} \rightarrow \phi e^{+} \nu_{e}$ decay, we show the distributions of the four kinematic variables. Contributions of the helicity form factors $H_{+}, H_{-}$and $H_{0}$ to the $q^{2}$ distributions are also displayed (Figure 9.1).

We remind the expressions of these form factors as function of the hadronic form factors 

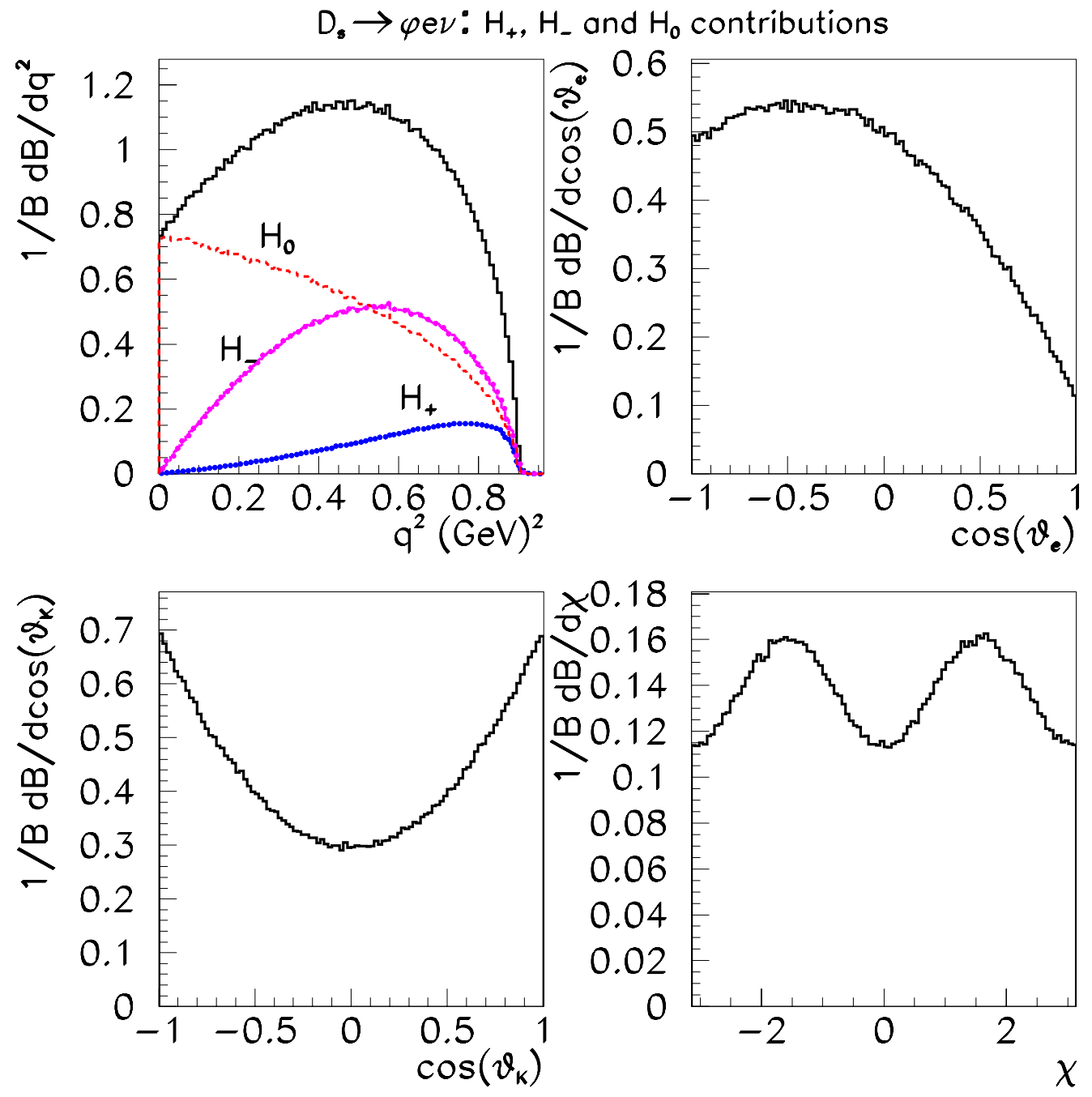

Figure 9.1: Contributions of the helicity form factors to the differential decay rate versus $q^{2}$ [H+ (blue), $H_{-}$(magenta), $H_{0}($ red) and total (black)] and angular distributions.

$A_{1}, A_{2}$ and $V$ :

$$
\begin{aligned}
H_{0}\left(q^{2}\right) & =\frac{1}{2 m_{\phi} q}\left[\left(m_{D s}^{2}-m_{\phi}^{2}-q^{2}\right)\left(m_{D s}+m_{\phi}\right) A_{1}\left(q^{2}\right)-4 \frac{m_{D s}^{2} p_{K K}^{2}}{m_{D s}+m_{\phi}} A_{2}\left(q^{2}\right)\right] \\
H_{ \pm}\left(q^{2}\right) & =\left(m_{D s}+m_{\phi}\right) A_{1}\left(q^{2}\right) \mp \frac{2 m_{D s} p_{K K}}{m_{D s}+m_{\phi}} V\left(q^{2}\right) .
\end{aligned}
$$

These plots are purely theoretical (once parameters are fixed to measured values) and are done with a toy experiment. Only the mass range between 1.01 and $1.03 \mathrm{GeV} / \mathrm{c}^{2}$ is considered 
and distributions are normalized to the number of generated events over the entire mass spectrum. In Figure 9.2, the projected angular distributions are given for each of the five $q^{2}$ bins as used in the fit. We oberve the same behaviour of the global distributions as function of the $q^{2}$ range as observed in the data (Figure 6.12): $\cos \theta_{\ell}$ and $\cos \theta_{V}$ have a specific shape at low $q^{2}$ and become rather uniform at high $q^{2}$, whereas the oscillations of $\chi$ are more pronounced at high $q^{2}$.

It is also possible to draw the contributions of $A_{1}$ and $V$ instead of $H_{+}$and $H_{-}$(Figure 9.3). Using these functions, we observe that the vector form factor has a really small contribution, versus $q^{2}$, which can explain the low sensitivity of the fit to the vector pole mass.

\subsection{Comparison with other experimental results}

Previous measurements of the form factors parameters for the $D_{s}^{+} \rightarrow \phi e^{+} \nu_{e}$ channel have been obtained in photo-production experiments, at Fermilab [73, 74, 76, 20], and by CLEOII [75]. Assuming a pole dominance for the different form factors with fixed values of the pole masses, they measure the ratios $r_{V}$ and $r_{2}$. Results have been summarized in Table 9.1. The only experiment that has measured a significant difference between $r_{V}$ and $r_{2}$ is FOCUS.

\begin{tabular}{|c|c|c|c|}
\hline Experiment & Statistics (S/B) & $r_{V}$ & $r_{2}$ \\
\hline E653 [73] & $19 / 5$ & $2.3_{-0.9}^{+1.1} \pm 0.4$ & $2.1_{-0.5}^{+0.6} \pm 0.2$ \\
\hline E687 [74] & $90 / 33$ & $1.8 \pm 0.9 \pm 0.2$ & $1.1 \pm 0.8 \pm 0.1$ \\
\hline CLEOII [75] & $308 / 166$ & $0.9 \pm 0.6 \pm 0.3$ & $1.4 \pm 0.5 \pm 0.3$ \\
\hline E791 [76] & $\sim 300 / 60$ & $2.27 \pm 0.35 \pm 0.22$ & $1.57 \pm 0.25 \pm 0.19$ \\
\hline FOCUS [20] & $\sim 560 / 250$ & $1.549 \pm 0.250 \pm 0.145$ & $0.713 \pm 0.202 \pm 0.266$ \\
\hline
\end{tabular}

Table 9.1: Results from previous experiments.

The parameters $r_{V}$ and $r_{2}$ have been also measured in the $D \rightarrow \bar{K}^{*} e^{+} \nu_{e}$ channel by different experiments and with an accuracy better than the previous $D_{s}^{+}$measurements thanks to the larger statistics available. Results from the PDG2006 [4] for the $D^{+} \rightarrow \bar{K}^{* 0} \ell^{+} \nu_{e}$ decay channel and the FOCUS result for $D^{0} \rightarrow K^{*-} \mu^{+} \nu_{\mu}$ are compared with our results (fixing pole masses to the same values) in Table 9.2. The accuracy of our measurement is similar to the one obtained for the world average $D$ decay, and it allows meaningful comparisons for the first time between $D^{+}$and $D_{s}^{+} \rightarrow V e \nu$ decays. Due to the $S U(3)$ symmetry, the form factors parameters are expected to be similar in these two channels as they mainly differ by the spectator quark. We observe that values for $r_{2}$ are identical within uncertainties but the agreement for $r_{V}$ is more marginal. 

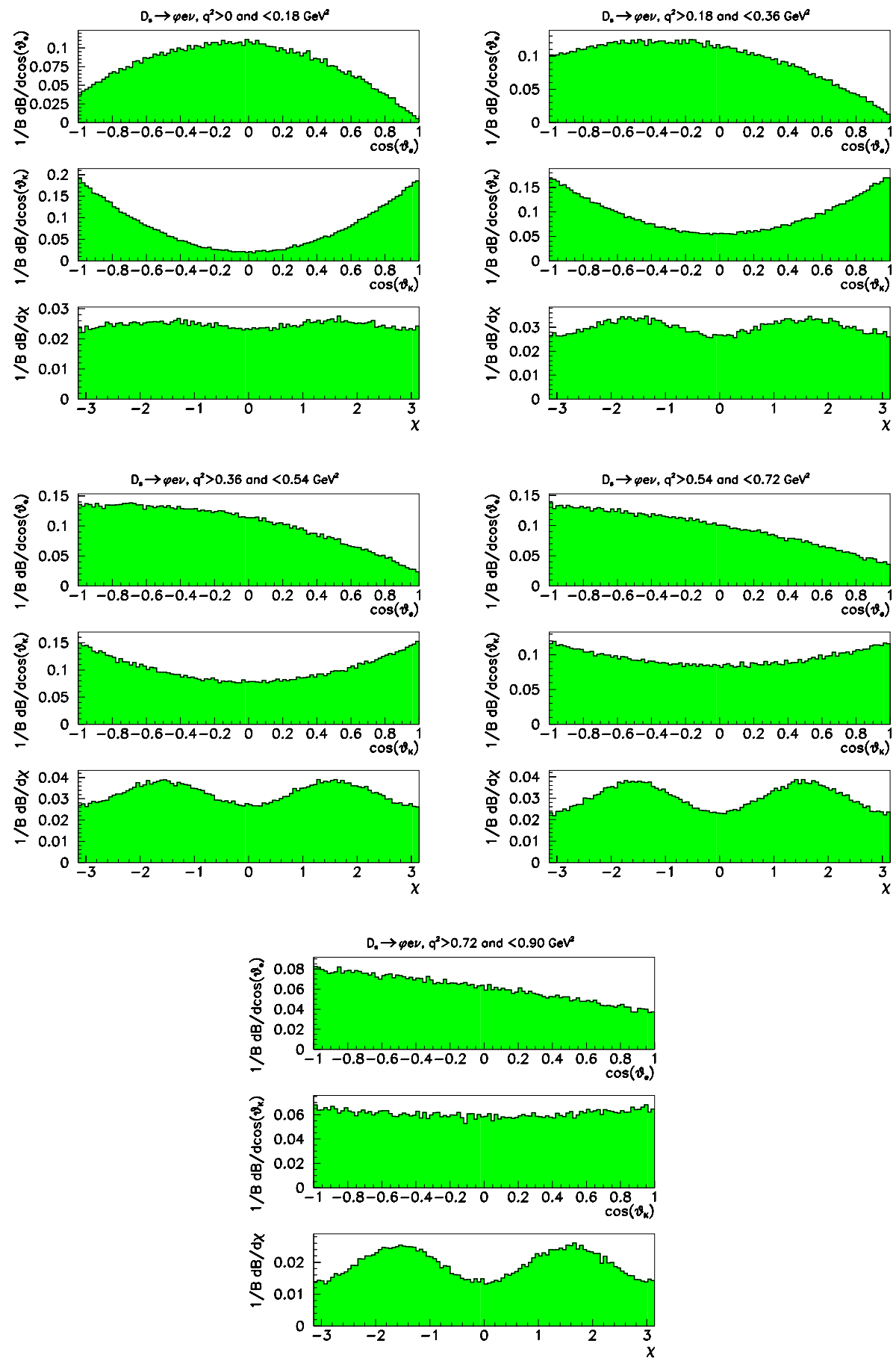

Figure 9.2: Distributions of $\cos \theta_{\ell}, \cos \theta_{V}$ and $\chi$ for different $q^{2}$ bins. Top left: $0<q^{2}<$ $0.18 \mathrm{GeV}^{2}$, top right: $0.18<q^{2}<0.36 \mathrm{GeV}^{2}$, middle left: $0.36<q^{2}<0.54 \mathrm{GeV}^{2}$, middle right: $0.54<q^{2}<0.72 \mathrm{GeV}^{2}$, bottom: $0.72<q^{2}<0.9 \mathrm{GeV}^{2}$. 

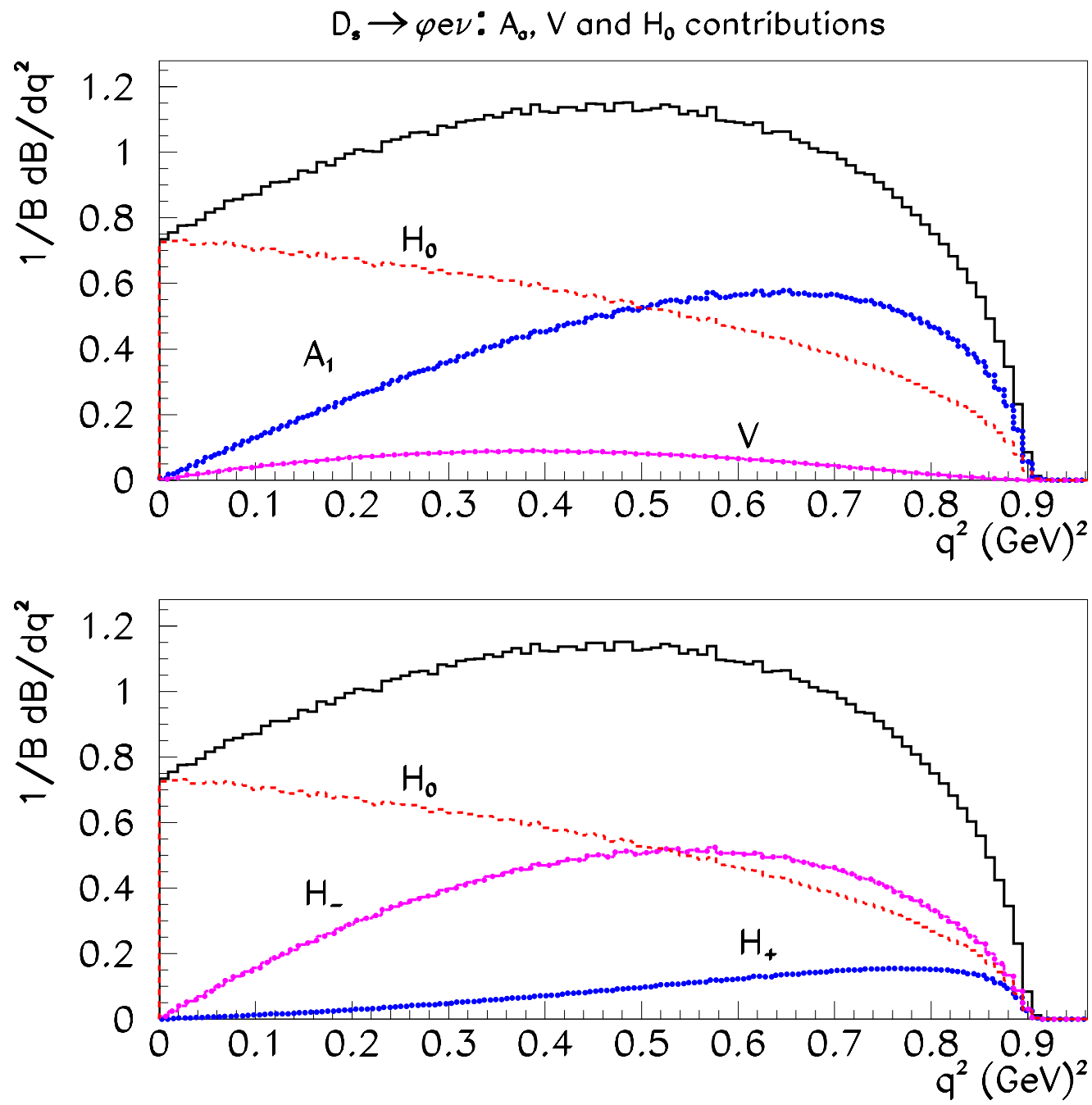

Figure 9.3: Contribution of the form factors $A_{1}$ (blue), $V$ (magenta), $H_{0}($ red) to the differential decay rate versus $q^{2}$ are compared with contributions from $H_{+}, H_{-}$and $H_{0}$.

The summary of experimental results is shown in Figure 9.4, where the previous measurements for the $D_{s}^{+} \rightarrow \phi e^{+} \nu_{e}$ channel are compared with the results of this analysis and with the $D^{+} \rightarrow \bar{K}^{* 0} \ell^{+} \nu$ channel. BABAR values are more accurate than previous determinations and compatible with most of them. 


\begin{tabular}{|c|c|c|c|}
\hline Parameter & $\begin{array}{c}D_{s}^{+} \rightarrow \phi e^{+} \nu_{e} \\
\text { (this analysis) }\end{array}$ & $\begin{array}{c}D^{+} \rightarrow K^{* 0} \ell^{+} \nu_{e} \\
(\mathrm{PDG} 06)\end{array}$ & $\begin{array}{c}D^{0} \rightarrow K^{*-} \mu^{+} \nu_{\mu} \\
\text { FOCUS [71] }\end{array}$ \\
\hline$r_{V}$ & $1.799 \pm 0.046 \pm 0.051$ & $1.62 \pm 0.08$ & $1.71 \pm 0.68 \pm 0.34$ \\
\hline$r_{2}$ & $0.813 \pm 0.036 \pm 0.030$ & $0.83 \pm 0.05$ & $0.91 \pm 0.37 \pm 0.10$ \\
\hline
\end{tabular}

Table 9.2: Comparison between $D / D_{s}^{+} \rightarrow V e^{+} \nu_{e}$ decays, for fixed values of the pole mass entering in the form factors.

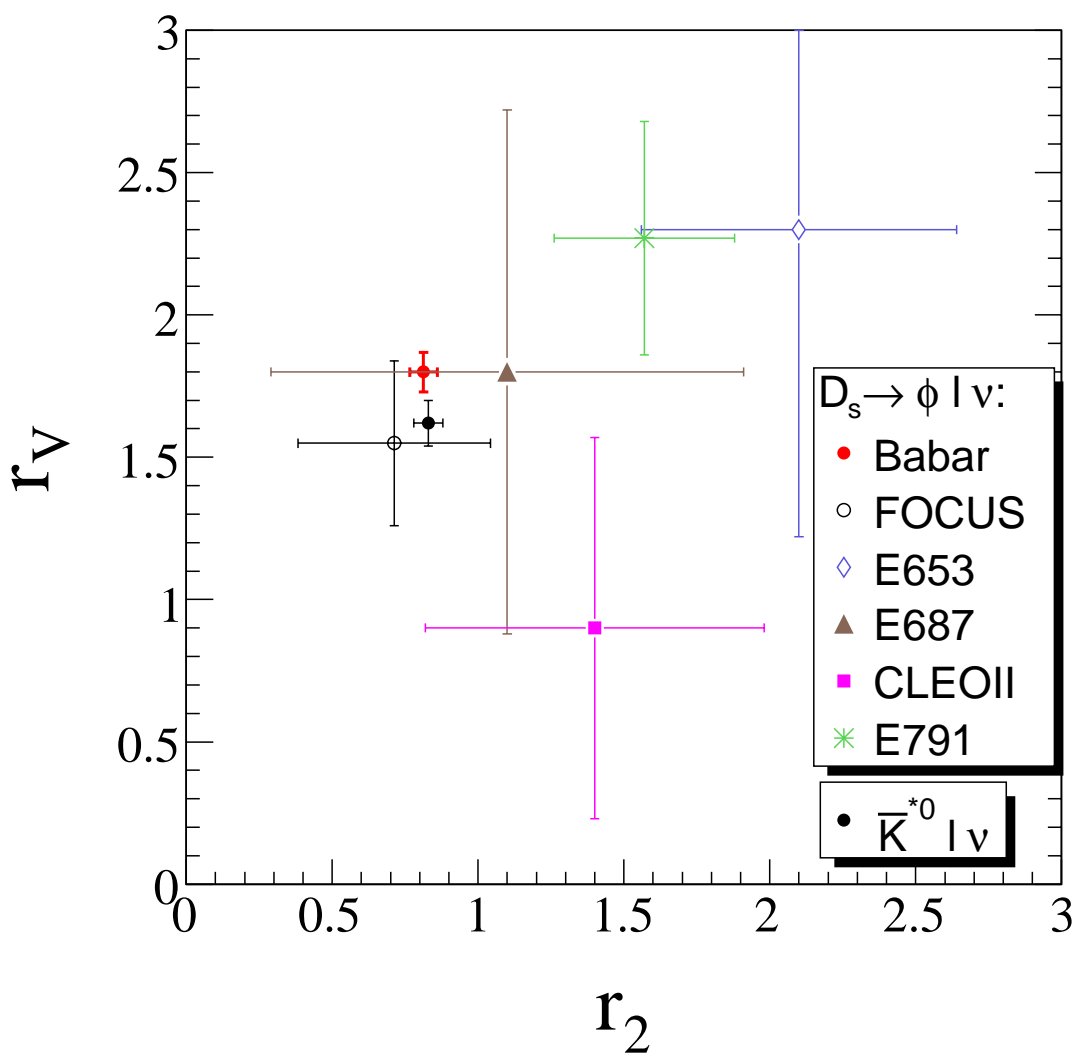

Figure 9.4: Results from previous experiments and present measurement of $r_{2}$ and $r_{V}$ in $D_{s}^{+} \rightarrow \phi e^{+} \nu_{e}$ decays. The error bars represent the statistical and systematic uncertainties added in quadrature. These measurements for $D_{s} \rightarrow \phi e^{+} \nu_{e}$ decays are compared with the world average of similar measurements obtained for $D \rightarrow \bar{K}^{*} e^{+} \nu_{e}$ decays [4]. 


\subsection{Comparison with theoretical calculations}

We compare the results obtained in this analysis (when $r_{0}, r_{2}, r_{V}$, and $m_{A}$ are fitted) to the theoretical expectations detailed in Section 1.3.2.

- Quark models: expectations from different quark models quoted in Table 1.3 are compared with the BABAR measurement in Figure 9.5. There is only one model ([35]) clearly compatible with the experimental results.

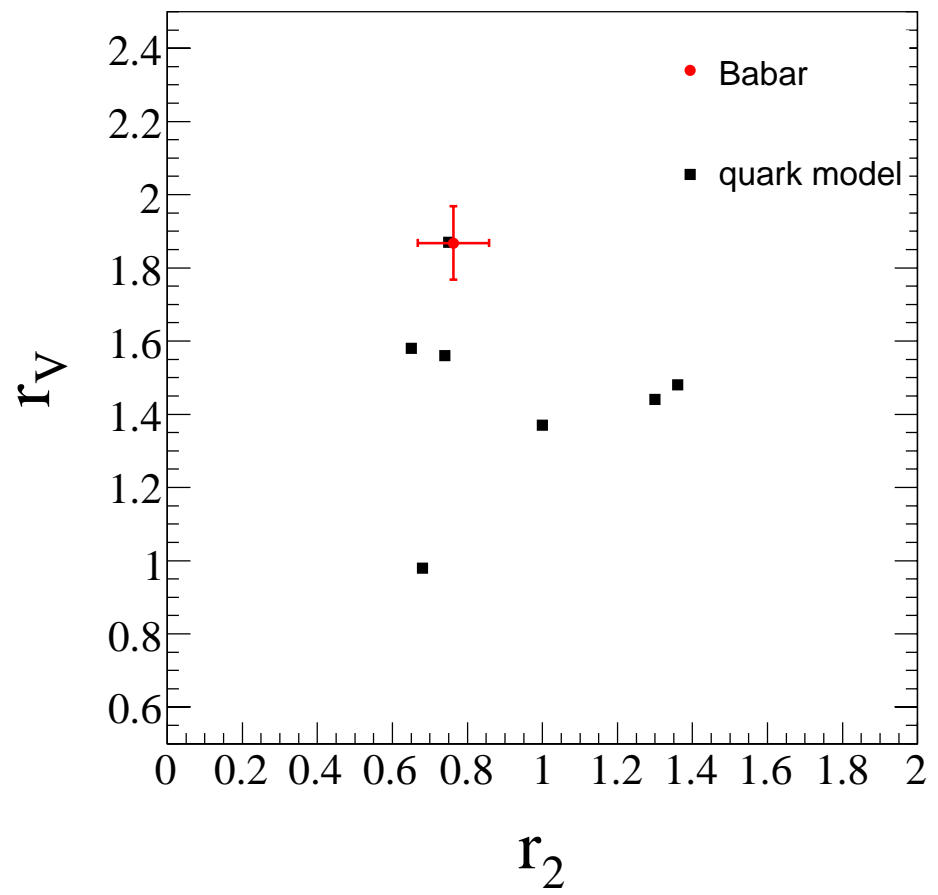

Figure 9.5: Comparison between BABAR results and quark model expectations.

- Lattice $Q C D$ : in Figure 9.6, our results are compared with the lattice calculations from [44], [43] (preliminary) and [45], which have been done in 1994, 2001 and 2002, respectively. These three calculations are performed in the quenched approximation. The two results of [45] correspond to two different values of the coupling. The calculation of [43] has been done for the $D_{s}^{+} \rightarrow \phi e^{+} \nu_{e}$ channel and for the $D \rightarrow \bar{K}^{*} e^{+} \nu_{e}$ whereas [44] and [45] correspond to the $D \rightarrow \bar{K}^{*} e^{+} \nu_{e}$ only. One can note that quoted uncertainties for the evaluation relative to $D_{s}^{+} \rightarrow \phi e^{+} \nu_{e}$ are smaller than those for $D \rightarrow \bar{K}^{*} e^{+} \nu_{e}$ but this quenched result does not agree with data. The BABAR determination of $r_{2}$ is compatible with all lattice results but we find a higher value of $r_{V}$ than the more recent lattice calculations. In [43], they also give the value of $A_{1}(0)=0.63_{-2}^{+2}$ for the $D_{s}^{+} \rightarrow \phi e^{+} \nu_{e}$ channel, which is in agreement with our measurement. 


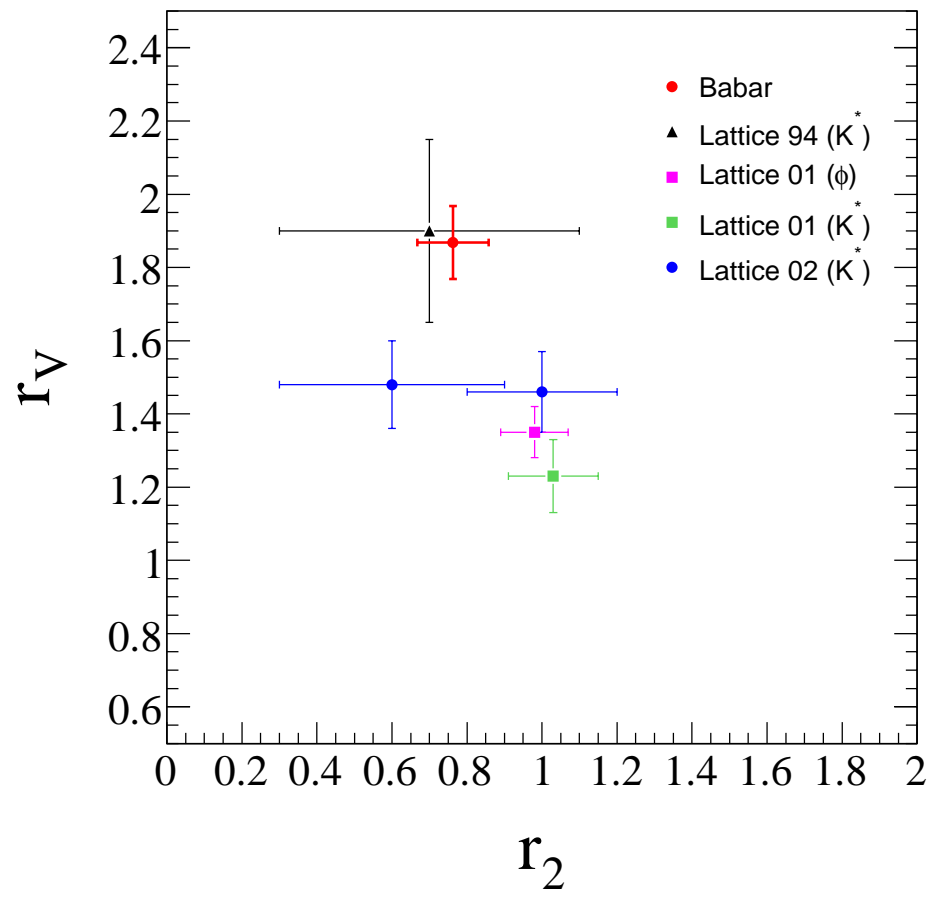

Figure 9.6: Comparison between BABAR results and lattice calculations using the quenched approximation. There is only one result, from 2001, corresponding to $D_{s}^{+} \rightarrow \phi e^{+} \nu_{e}$ decay channel, the others correspond to $D \rightarrow \bar{K}^{*} e^{+} \nu_{e}$.

- QCD sum rules: results from QCD sum rules are shown in Figure 9.7, using values from [39] and [40]. The uncertainties of these two determinations are very large. They are compatible with our results.

- Fajfer-Kamenik ansatz: we compare our results with the values of the form factors parameters given in [48] (see Eq. (1.84) to (1.87)). Their value of $r_{V}=1.8$, which derives from a constraint of LEET, is in agreement with our result, but they find $r_{2}=0.52$ which is much lower than the experimental determination. They also give $A_{1}(0)=0.61$ and $b^{\prime}=0.74$ that corresponds to $m_{A}=2.43 \mathrm{GeV} / \mathrm{c}^{2}$. These last values agree with our determination.

- z parameterization: in Figures 9.8,9.9 and 9.10 we have displayed the variation versus $-z$ of the different form factors, normalized to 1 at $q^{2}=0$ after having removed the contribution from the $z$ expansion and considering cases were we take no or one pole (see Section 1.3.1 and reference [31]). We use $-z$ as variable so that the different form factors have a positive slope, as they have when displayed versus $q^{2}$. We compare these expectations with the simple pole model using our fitted values.

Poles which have been considered are the $D_{s}^{*}(2112)$ for the vector form factor and 


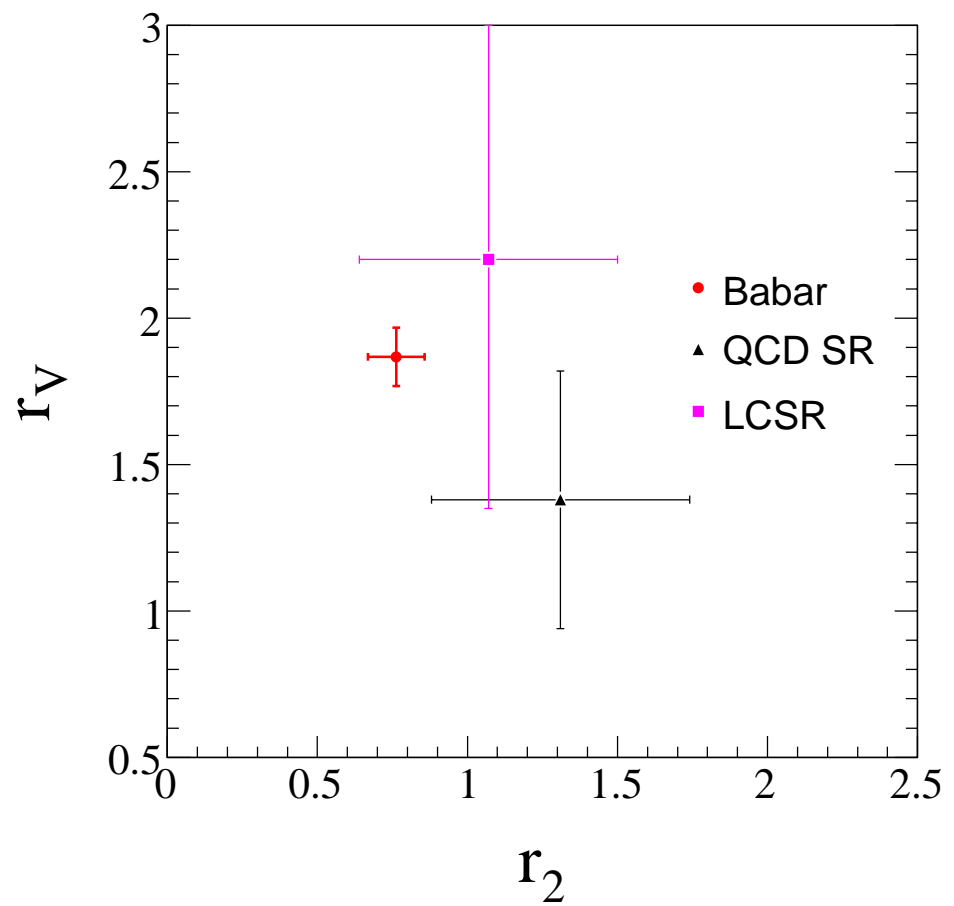

Figure 9.7: Comparison between BABAR results and expectations from QCD sum rules[39], and light cone sum rules [40].

the $D_{s 1}$ state at $2459 \mathrm{MeV} / \mathrm{c}^{2}$ for the axial-vector form factors. We observe that the form factors expressed with this parameterization have a quasi-linear dependence. When compared with a simple pole parameterization one note that it has a quite similar behaviour and that a linear expansion in $z$ can account for the difference. In particular, for the vector form factor, the agreement between the simple pole model and the $z$ parameterization with one pole is already very good. 


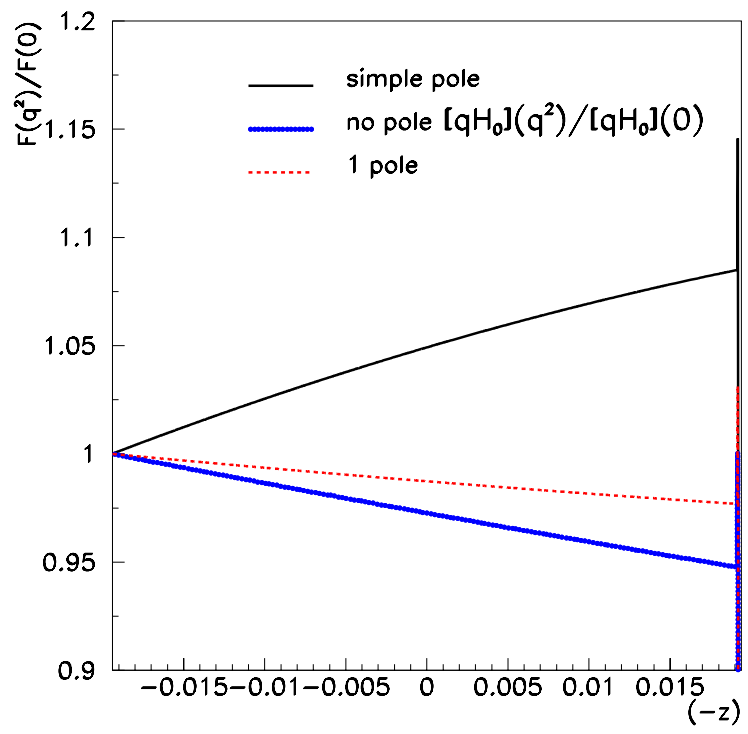

Figure 9.8: Variation of the $q H_{0}\left(q^{2}\right)$ form factor parameterization versus - z, not including the $z$ expansion and considering that no, or one pole contributes. These distributions are compared with the simple pole parameterization.

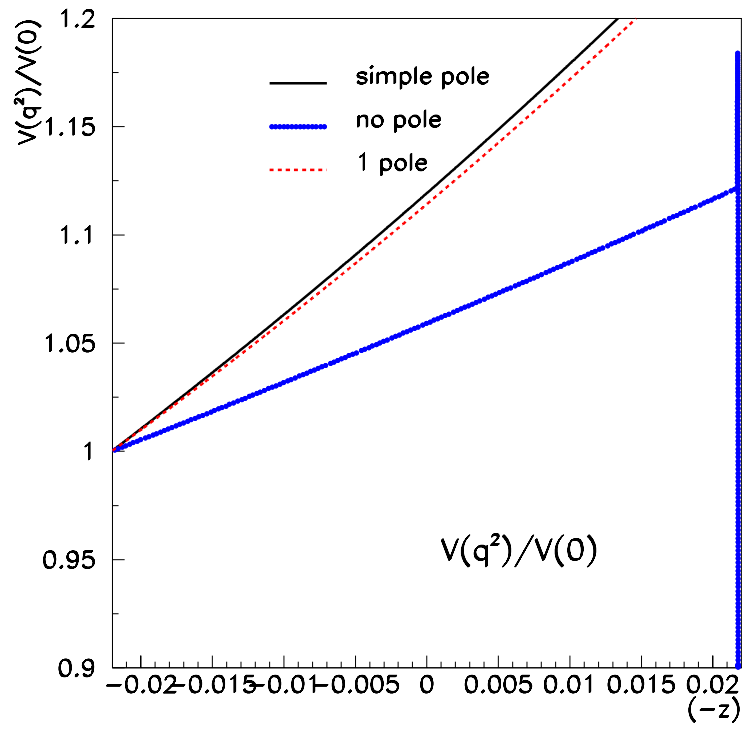

Figure 9.9: Variation of the $V\left(q^{2}\right)$ form factor parameterization versus $-z$, not including the $z$ expansion and considering that no, or one pole contributes. These distributions are compared with the simple pole parameterization. 


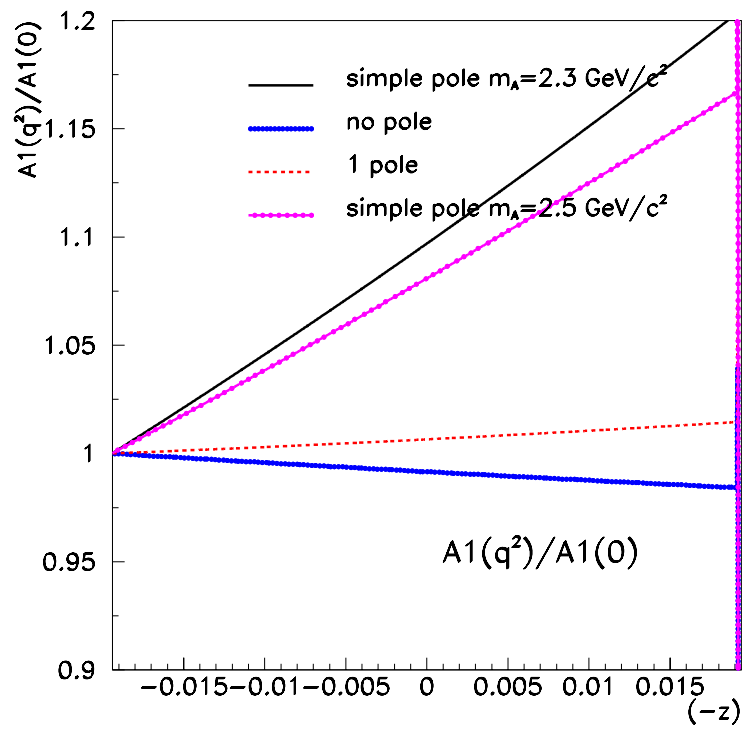

Figure 9.10: Variation of the $A_{1}\left(q^{2}\right)$ form factor parameterization versus $-z$, not including the $z$ expansion and considering that no, or one pole contributes. These distributions are compared with the simple pole parameterization. 


\subsection{Present measurements and HQET}

We have measured the respective contributions of the three hadronic form factors in the decay $D_{s}^{+} \rightarrow \phi e^{+} \nu_{e}$. Using the formalism given in [27] which provides expressions for the first order corrections expected from pertubative QCD and $1 / m$ expansion, we have evaluated the relative importance of these effects. In the original publication, from 1992, experimental results were too uncertain so that definite conclusions could be drawn.

At zero order we remind the following expectations from HQET for the values of the different form factors at $q^{2}=q_{\max }^{2}$. (or $w=1$ ):

$$
f_{+}\left(q_{\text {max. }}^{2}\right)=V\left(q_{\text {max. }}^{2} .\right)=A_{2}\left(q_{\text {max. }}^{2}\right)=R^{-2} A_{1}\left(q_{\text {max. }}^{2}\right)=R^{-1} \xi\left(q_{\text {max. }}^{2}\right) \simeq 1.1 .
$$

At this order $\xi\left(q_{\text {max. }}^{2}\right)=1$ and $R=\frac{2 \sqrt{m_{H} m_{P(V)}}}{m_{H}+m_{P(V)}}=0.95$ for $D_{s}^{+} \rightarrow \phi e^{+} \nu_{e}$ and $=0.81$ for $D \rightarrow K e \nu_{e}$. These expectations are compared with present measurements in Table 9.3.

\begin{tabular}{|c|c|c|c|c|}
\hline & $f_{+}\left(q_{\max .}^{2}\right)$ & $V\left(q_{\max .}^{2}\right)$ & $A_{2}\left(q_{\max .}^{2}\right)$ & $A_{1}\left(q_{\max .}^{2}\right)$ \\
\hline HQET $0^{\text {th. }}$ order & 1.23 & 1.05 & 1.05 & 0.95 \\
\hline Measurements & $1.53 \pm 0.05$ & $1.42 \pm 0.11$ & $0.56 \pm 0.07$ & $0.73 \pm 0.05$ \\
\hline
\end{tabular}

Table 9.3: Quoted values for the measurements are obtained by extrapolating to $q_{m a x}^{2}$. the values given at $q^{2}=0$, using the measured $q^{2}$ variation of the form factors, in the framework of the simple pole ansatz.

The value quoted for the measurement of $f_{+}\left(q_{\text {max }}^{2}\right)$ corresponds to the BABAR result obtained with $D^{0} \rightarrow K^{-} e^{+} \nu_{e}$. Table 9.3 indicates that corrections to naive HQET expectations are large, varying from 30 to $50 \%$. In the following we examine if, including first order corrections, a general picture emerges.

\subsubsection{Determination of QCD corrections}

To determine the parameter $\alpha_{s}$ characterizing the perturbative QCD corrections, we use the form factor $A_{1}$. First order corrections (QCD corrections and finite mass corrections) to the form factor $A_{1}$ can be expressed as [27]:

$$
A_{1}(w)=R \frac{w+1}{2}\left[\alpha_{A_{1}}(w) X_{Q C D}(w) \xi(w)+\frac{\bar{\Lambda}}{2 m_{s}}\left(\frac{w-1}{w+1} \xi(w)+\Psi_{A_{1}}(w)\right)\right]
$$


where

$$
\alpha_{A_{1}}(w)=1+\frac{\alpha_{S}}{\pi} \tilde{\beta}_{A_{1}}(w)
$$

and

$$
\Psi_{A_{1}}(w)=\psi_{1}(w)-2 \psi_{3}(w)
$$

The functions $\tilde{\beta}_{A_{1}}(w)$ and $X_{Q C D}(w)$ have been evaluated in QCD whereas the Isgur-Wise function $\xi(w)$ and the two other functions which are introduced when considering the first order $1 / m_{q}$ correction are unknown. At $w=1$, constraints on normalization and on the contribution from first order corrections apply:

$$
\xi(1)=1, \psi_{1}(1)=\psi_{3}(1)=0 .
$$

The last equalities reflect the Luke theorem according to which $1 / m$ corrections disappear for some of the hadronic form factors when $w \rightarrow 1$. Equation (9.6) is not valid at order $1 / m_{q}^{2}$.

At $w=1,1 / m$ corrections in Equation (9.3) disappear and one expects that the value of $A_{1}(1)$ is modified only by QCD corrections:

$$
A_{1}(1)=R \alpha_{A_{1}}(1) X_{Q C D}(1)=1.20-2.0 \frac{\alpha_{S}}{\pi} .
$$

Numerical values for $X_{Q C D}(1)=1.27$ and $\tilde{\beta}_{A_{1}}(1)=-1.65$ are given in [27]. Comparing the expected value for $A_{1}(1)$ with the experimental result one obtains:

$$
\frac{\alpha_{S}}{\pi}=0.24 \pm 0.03
$$

This value corresponds to an effective scale of the order of $500 \mathrm{MeV}$. In the original study [27], larger corrections were obtained from first order perturbative QCD based on the measurement available at that time $\left(A_{1}(1) \simeq 0.58 \pm 0.06\right.$ for $\left.D \rightarrow K^{*} e^{+} \nu_{e}\right)$. We are in a domain where the validity of perturbative QCD evaluations is marginal and one can only expect to have an approximate picture of the mechanisms at work. 


\subsubsection{Determination of $\bar{\Lambda} / 2 m_{s}$}

The parameter $\bar{\Lambda} / 2 m_{s}$ characterizes the scale of $1 / m$ corrections. In the formalism used to evaluate these corrections, $\bar{\Lambda}$ corresponds to the difference between the masses of hadrons and quarks:

$$
m_{c}=\frac{1}{4}\left(3 m_{D^{*}}+m_{D}\right)-\bar{\Lambda} ; m_{s}=\frac{1}{4}\left(3 m_{K^{*}}+m_{K}\right)-\bar{\Lambda}
$$

We use the form factor $V$ to determine $\bar{\Lambda} / 2 m_{s}$. First order corrections to the form factor $V$ can be expressed as [27]:

$$
V(w)=R^{-1}\left\{\alpha_{V}(w) X_{Q C D}(w) \xi(w)+\frac{\bar{\Lambda}}{2 m_{s}}\left[\xi(w)+\Psi_{V}(w)\right]\right\}
$$

where

$$
\alpha_{V}(w)=1+\frac{\alpha_{S}}{\pi} \tilde{\beta}_{V}(w)
$$

and

$$
\Psi_{V}(w)=\psi_{1}(w)-2 \psi_{3}(w)
$$

One can note that the unknown function $\Psi_{V}(w)=\Psi_{A_{1}}(w)$ is equal to zero when $w=1$. It can be noted also that the ratio between the two form factors at $w=1$ is independent of the value of $\xi(1)$.

$$
r_{V}\left(q_{\text {max. }}^{2}\right)=R^{-2} \frac{\alpha_{V}(1) X_{Q C D}(1)+\frac{\bar{\Lambda}}{2 m_{s}}}{\alpha_{A_{1}}(1) X_{Q C D}(1)}
$$

Using the value $\tilde{\beta}_{V}(1)=-0.315$ [27] and the previous determination of $\frac{\alpha_{S}}{\pi}$ this gives:

$$
\begin{aligned}
\frac{\bar{\Lambda}}{2 m_{s}} & =X_{Q C D}(1)\left\{R^{2} r_{V}\left(q_{m a x .}^{2}\right)-1+\frac{\alpha_{S}}{\pi}\left[R^{2} r_{V}\left(q_{m a x}^{2} \tilde{\beta}_{A_{1}}(1)-\tilde{\beta}_{V}(1)\right]\right\}\right. \\
& =0.18 \pm 0.13
\end{aligned}
$$

Using the expression in Equation (9.9) relating the effective strange quark mass and $\bar{\Lambda}$ one 
obtains:

$$
\bar{\Lambda}=(0.2 \pm 0.1) \mathrm{GeV} \text { and } m_{s}=(0.6 \pm 0.1) \mathrm{GeV} / \mathrm{c}^{2}
$$

In [27], they obtain: $\frac{\bar{\Lambda}}{2 m_{s}}=-0.05 \pm 0.24$ and $\bar{\Lambda}=(-44 \pm 297) \mathrm{MeV}$; uncertainties were too large to draw any conclusions. The parameter $\bar{\Lambda}$ is universal and can be compared with a determination obtained in $B$ meson semileptonic decays.

These numerical evaluations provide an explanation for the rise of the value of $r_{V}\left(q_{\max .}^{2}\right)$ from 1.1 (zeroth order expectation) to 1.94 (measured value). Perturbative QCD corrections decrease $A_{1}$ by $30 \%$ and increase $V$ by $20 \%$. Another increase of $V$ by $20 \%$ is given by the $1 / m$ correction. The determination of $1 / m$ corrections shows that their typical size is similar (or lower) as compared with first order QCD terms.

\subsubsection{Evaluation of the corrections to $f_{+}\left(q_{\max .}^{2}\right)$ and $A_{2}\left(q_{\text {max. }}^{2}\right)$}

The decay $D \rightarrow K^{-} e^{+} \nu_{e}$ depends on a single hadronic vector form factor $\left(f_{+}\left(q^{2}\right)\right)$. First order corrections have been evaluated in [27]:

$$
f_{+}(w)=R^{-1}\left\{\alpha_{f}(w) X_{Q C D}(w) \xi(w)+\frac{\bar{\Lambda}}{2 m_{s}}\left[\frac{m_{K}-m_{D}}{m_{K}+m_{D}}(w-2) \xi(w)+\Psi_{f}(w)\right]\right\}
$$

where

$$
\alpha_{f}(w)=1+\frac{\alpha_{S}}{\pi}\left[\tilde{\beta}_{+}(w)+\frac{m_{K}-m_{D}}{m_{K}+m_{D}} \tilde{\beta}_{-}(w)\right]
$$

and

$$
\Psi_{f}(w)=\psi_{1}(w)-2(w-1) \psi_{2}(w)+6 \psi_{3}(w)-\frac{m_{K}-m_{D}}{m_{K}+m_{D}}(w+1) \psi_{+}(w)
$$

At $w=1$, these expressions simplify to:

$$
f_{+}(1)=R^{-1}\left\{\alpha_{f}(1) X_{Q C D}(1)+\frac{\bar{\Lambda}}{2 m_{s}} \frac{m_{D}-m_{K}}{m_{K}+m_{D}}\left[1+2 \psi_{+}(1)\right]\right\}
$$

Using $\tilde{\beta}_{+}(1)=-0.98$ and $\tilde{\beta}_{-}(1)=-0.26$ from [27] and the previous determinations of $\frac{\alpha_{S}}{\pi}$ and $\frac{\bar{\Lambda}}{2 m_{s}}$, a value is obtained for $\psi_{+}(1)$ by comparing the measured and expected values of 
$f_{+}(1)$.

$$
f_{+}(1)=1.23\left[1.02+0.58 *(0.18 \pm 0.13)\left(1+2 \psi_{+}(1)\right)\right]=1.53 \pm 0.05
$$

giving a rather undetermined value $\psi_{+}(1)=0.6 \pm 0.8$. It can be observed that perturbative QCD corrections do not change significantly the value of $f_{+}\left(q_{\text {max }}^{2}\right.$. $)$ and that the $1 / m$ term is necessary to have agreement between measured and expected values of the form factor.

A similar study can be done for the $A_{2}$ form factor:

$$
A_{2}(w)=R^{-1}\left\{\alpha_{A_{2}}(w) X_{Q C D}(w) \xi(w)+\frac{\bar{\Lambda}}{2 m_{s}}\left[-\frac{m_{\phi}}{m_{D_{s}}} \xi(w)+\Psi_{A_{2}}(w)\right]\right\}
$$

where

$$
\alpha_{A_{2}}(w)=1+\frac{\alpha_{S}}{\pi}\left(\frac{m_{\phi}}{m_{D_{s}}} \tilde{\beta}_{A_{2}}(w)+\tilde{\beta}_{A_{3}}(w)\right)
$$

and

$$
\Psi_{A_{2}}(w)=\psi_{1}(w)+2\left(\frac{m_{\phi}}{m_{D_{s}}}-1\right) \psi_{2}(w)-2 \psi_{3}(w)+\left(\frac{m_{\phi}}{m_{D_{s}}}+1\right) \psi_{+}(w) .
$$

At $w=1$, these expressions simplifies to:

$$
A_{2}(1)=R^{-1}\left\{\alpha_{A_{2}}(1) X_{Q C D}(1)+\frac{\bar{A}}{2 m_{s}}\left[-\frac{m_{\phi}}{m_{D_{s}}}+2\left(\frac{m_{\phi}}{m_{D_{s}}}-1\right) \psi_{2}(1)+\left(\frac{m_{\phi}}{m_{D_{s}}}+1\right) \psi_{+}(1)\right]\right\}
$$

Using $\tilde{\beta}_{A_{2}}(1)=-1.20$ and $\tilde{\beta}_{A_{3}}(1)=-1.23$ from [27] and the previous determinations of $\frac{\alpha_{S}}{\pi}$ and $\frac{\bar{\Lambda}}{2 m_{s}}$, one can relate the values of for $\psi_{+}(1)$ and $\psi_{2}(1)$ by comparing the measured and expected values of $A_{2}(1)$.

$$
\begin{aligned}
A_{2}(1) & =1.05\left[(0.71 \pm 0.07)+(0.18 \pm 0.13)\left(-0.52-0.96 \psi_{2}(1)+1.52 \psi_{+}(1)\right)\right] \\
& =0.56 \pm 0.07
\end{aligned}
$$

The diminution of $A_{2}(1)$ as compared with the zero-order estimate comes from a $30 \%$ decrease from pertubative QCD and an additionnal decrease of $20 \%$ from $1 / m$ corrections. 


\subsection{Experimental results on charm semileptonic decays branching fractions}

\subsubsection{Inclusive results}

Before the first measurements from the CLEO-c collaboration [77], inclusive semileptonic branching fractions of charm mesons were rather inaccurate as there was no easy way to tag the production of a $D^{0}$, a $D^{+}$or a $D_{s}^{+}$meson.

\begin{tabular}{|c|c|c|}
\hline & $D^{0}$ & $D^{+}$ \\
\hline Inclusive s.l. BR (\%)[77] & $6.46 \pm 0.17 \pm 0.13$ & $16.13 \pm 0.20 \pm 0.33$ \\
Lifetime $(p s)[4]$ & $0.4101 \pm 0.0015$ & $1.040 \pm 0.007$ \\
Inclusive s.l. width $\left(\times 10^{-2} \mathrm{ps}^{-1}\right)$ & $15.75 \pm 0.41 \pm 0.32$ & $15.51 \pm 0.20 \pm 0.31$ \\
\hline
\end{tabular}

Table 9.4: Inclusive results on $D^{0}$ and $D^{+}$mesons.

Results from CLEO-c [77] for $D^{(0,+)}$ are given in Table 9.4. These values are consistent with isospin invariance: $\Gamma_{D^{+}}^{s l} / \Gamma_{D^{0}}^{s l}=0.985 \pm 0.028 \pm 0.015$. Isospin invariance can be broken mainly in Cabibbo suppressed semileptonic decays.

For the $D_{s}^{+}$, similar results are not available yet, the inclusive semileptonic branching fraction quoted in [4] being $8_{-5}^{+6} \%$. Using the measured inclusive semileptonic width for non-charmed $D$-mesons, the measured $D_{s}^{+}$lifetime $-(0.500 \pm 0.007)$ ps- and assuming SU(3) invariance, one expects:

$$
B R_{D_{s}}^{s l .}=7.8 \%
$$

One can note that in $D_{s}$ semileptonic decays there could be contributions from $s \bar{s}$ annihilation, a mechanism which is not possible in $D^{0}$ or $D^{+}$decays (apart in Cabibbo-suppressed $D^{+}$transitions).

So, prior to a real measurement, there are still large uncertainties on the inclusive semileptonic decay branching fraction of $D_{s}$ mesons.

\subsubsection{Exclusive measurements}

Rather accurate measurements exist also for exclusive $D^{(0,+)}$ semileptonic decay channels. Cabibbo allowed individual measurements are compatible with isospin invariance.

The sum of exclusive semileptonic $D^{(0,+)}$ decay channels agrees with the inclusive results so the "missing" channels are compatible with present uncertainties which are of the order of $3 \%$ (relative). 


\begin{tabular}{|c|c|c|c|c|c|}
\hline $\begin{array}{c}D^{0} \text { decay } \\
\text { channel }\end{array}$ & $\begin{array}{c}\text { BR } \\
(\%)\end{array}$ & $\begin{array}{c}\Gamma_{D^{0}}^{\text {sl. }} \\
\left(\times 10^{-2} p s^{-1}\right)\end{array}$ & $\begin{array}{c}D^{+} \text {decay } \\
\text { channel }\end{array}$ & $\begin{array}{c}\text { BR } \\
(\%)\end{array}$ & $\begin{array}{c}\Gamma_{D^{+}}^{\text {sl. }} \\
\left(\times 10^{-2} p s^{-1}\right)\end{array}$ \\
\hline$K^{-} e^{+} \nu_{e}$ & $3.53 \pm 0.05$ & $8.61 \pm 0.12$ & $\overline{K^{0}} e^{+} \nu_{e}$ & $8.55 \pm 0.23$ & $8.22 \pm 0.22$ \\
$K^{*-} e^{+} \nu_{e}$ & $2.17 \pm 0.16$ & $5.29 \pm 0.39$ & $\overline{K^{* 0}} e^{+} \nu_{e}$ & $5.61 \pm 0.31$ & $5.39 \pm 0.30$ \\
$(K \pi)_{S}^{-} e^{+} \nu_{e}$ & & & $\frac{(K \pi)_{S}^{0} e^{+} \nu_{e}}{0.3 \pm 0.1}$ & $0.3 \pm 0.1$ \\
$K_{1}^{-}(1270) e^{+} \nu_{e}$ & $0.08_{-0.03}^{+0.04}$ & $0.2 \pm 0.1$ & $\frac{K_{1}^{0}}{(1270) e^{+} \nu_{e}}$ & & \\
$K_{1}^{*-}(1400) e^{+} \nu_{e}$ & & & $\frac{K_{1}^{* 0}}{K_{2}^{* 0}}(1400) e^{+} \nu_{e}$ & & \\
$K_{2}^{*-}(1430) e^{+} \nu_{e}$ & & & $\pi^{0} e^{+} \nu_{e}$ & $0.380 \pm 0.024$ & $0.37 \pm 0.02$ \\
\hline$\pi^{-} e^{+} \nu_{e}$ & $0.293 \pm 0.011$ & $0.71 \pm 0.03$ & $\rho^{0} e^{+} \nu_{e}$ & $0.22 \pm 0.04$ & $0.21 \pm 0.04$ \\
$\rho^{-} e^{+} \nu_{e}$ & $0.19 \pm 0.04$ & $0.46 \pm 0.10$ & $\omega^{0} e^{+} \nu_{e}$ & $0.16_{-0.06}^{+0.07}$ & $0.15 \pm 0.07$ \\
\hline Total measured & $6.26 \pm 0.18$ & $15.26 \pm 0.44$ & & $15.22 \pm 0.41$ & $14.63 \pm 0.39$ \\
\hline
\end{tabular}

Table 9.5: Exclusive results on $D^{0}$ and $D^{+}$mesons.

\begin{tabular}{|c|c|c|c|c|}
\hline $\begin{array}{c}D_{s}^{+} \text {decay } \\
\text { channel }\end{array}$ & $\begin{array}{c}\Gamma_{D}^{s l .} \\
\left(\times 10^{-2} p s^{-1}\right)\end{array}$ & $\begin{array}{c}D_{s}^{+} \text {BR expected } \\
\mathrm{SU}(3)(\%)\end{array}$ & $\begin{array}{c}\text { BR (PDG06) } \\
(\%)\end{array}$ & $\begin{array}{c}\text { BR (ISGW2) } \\
(\%)\end{array}$ \\
\hline$\left(\eta+\eta^{\prime}\right) e^{+} \nu_{e}$ & $8.52 \pm 0.11$ & $4.3 \pm 0.1$ & $4.2 \pm 0.8$ & 3.3 \\
$\phi e^{+} \nu_{e}$ & $5.35 \pm 0.24$ & $2.68 \pm 0.13$ & $2.4 \pm 0.4$ & 2.3 \\
$f_{0} e^{+} \nu_{e}$ & $0.3 \pm 0.1$ & $0.15 \pm 0.05$ & & 0.0 \\
$K^{0} e^{+} \nu_{e}$ & $0.71 \pm 0.03$ & $0.36 \pm 0.02$ & & 0.22 \\
$K^{* 0} e^{+} \nu_{e}$ & $0.46 \pm 0.10$ & $0.23 \pm 0.05$ & & 0.11 \\
\hline
\end{tabular}

Table 9.6: Expectations for $D_{s}^{+}$exclusive decays assuming $\mathrm{SU}(3)$.

Cabibbo allowed decays are dominated by the $K / K^{*} e^{+} \nu_{e}$ channels. Contributions from other excited states as the $0^{+}$and $1^{+}$have been recently observed, at a very low level $(1-2 \%$ of the total width for each channel). Dominance of the pseudo-scalar and vector hadronic final states is expected from HQET and it is surprising that it is verified in $D$ decays even better than in $B$ decays. Naive expectations were in the opposite direction.

From these results, and assuming SU(3) symmetry, one can evaluate expectations for $D_{s}^{+}$ semileptonic decays and compare with other models. Table 9.6 gives this comparison. The ISGW2 model predicts rather large violations of SU(3) symmetry. Our result, $B R\left(D_{s}^{+} \rightarrow\right.$ $\left.\phi e^{+} \nu_{e}\right)=(2.585 \pm 0.031 \pm 0.075 \pm 0.147) \times 10^{-2}$, is in agreement with the value expected from universality of semileptonic decay widths of charm mesons. 


\subsection{Evaluation of $D_{s}^{+} \rightarrow f_{0} e^{+} \nu_{e}$}

Taking the $f_{0}$ as a candidate for the $S$-wave contribution, we measure $r_{0}=(15.3 \pm 2.6 \pm$ 1) $\mathrm{GeV}^{-1}$, which corresponds, in the $K^{+} K^{-}$mass range between 1.01 and $1.03 \mathrm{GeV} / \mathrm{c}^{2}$, to the ratio:

$$
\frac{B R\left(D_{s} \rightarrow f_{0} e^{+} \nu_{e}\right) \times B R\left(f_{0} \rightarrow K^{+} K^{-}\right)}{B R\left(D_{s} \rightarrow K^{+} K^{-} e^{+} \nu_{e}\right)}=0.23_{-0.08}^{+0.12} \pm 0.03 \%
$$

Using the $f_{0}$ decay parameters measured by BES, the $K^{+} K^{-}$and $\pi^{+} \pi^{-}$mass distributions given in Figure 9.11 are expected for the decay $D_{s} \rightarrow f_{0} e^{+} \nu_{e}$. The following value for the ratio between these two decay channels is expected:

$$
\frac{B R\left(D_{s} \rightarrow f_{0} e^{+} \nu_{e}\right) \times B R\left(f_{0} \rightarrow K^{+} K^{-}\right)}{B R\left(D_{s} \rightarrow f_{0} e^{+} \nu_{e}\right) \times B R\left(f^{0} \rightarrow \pi^{+} \pi^{-}\right)}=0.345
$$

These evaluations can be checked by searching for the decay channel $D_{s} \rightarrow \pi^{+} \pi^{-} e^{+} \nu_{e}$ which is expected to be dominated by the $f_{0}$ contribution.

One can also compare the $S$-wave contribution relatively to the $P$-wave in the $D_{s}^{+} \rightarrow$ $K^{+} K^{-} e^{+} \nu_{e}$ and in $D^{+} \rightarrow K^{-} \pi^{+} \ell \nu$. Still considering the $f_{0}$ as candidate for the $S$-wave, and correcting for efficiency effects, we obtain for the entire mass range:

$$
\frac{B R\left(D_{s} \rightarrow f_{0} e^{+} \nu_{e}\right)}{B R\left(D_{s} \rightarrow \phi e^{+} \nu_{e}\right)}=4.7_{-1.6}^{+2.5} \pm 0.6 \%
$$

In the $K \pi$ mass range [0.65, 1.5$] \mathrm{GeV} / \mathrm{c}^{2}$, the FOCUS collaboration has measured [72]:

$$
\frac{B R\left(D \rightarrow K \pi \mu \nu_{\mu}\right)_{S}}{B R\left(D \rightarrow K \pi \mu \nu_{\mu}\right)_{P}}=5.30 \pm 0.74_{-0.96}^{+0.99} \%
$$

The ratio is similar for the two channels. 


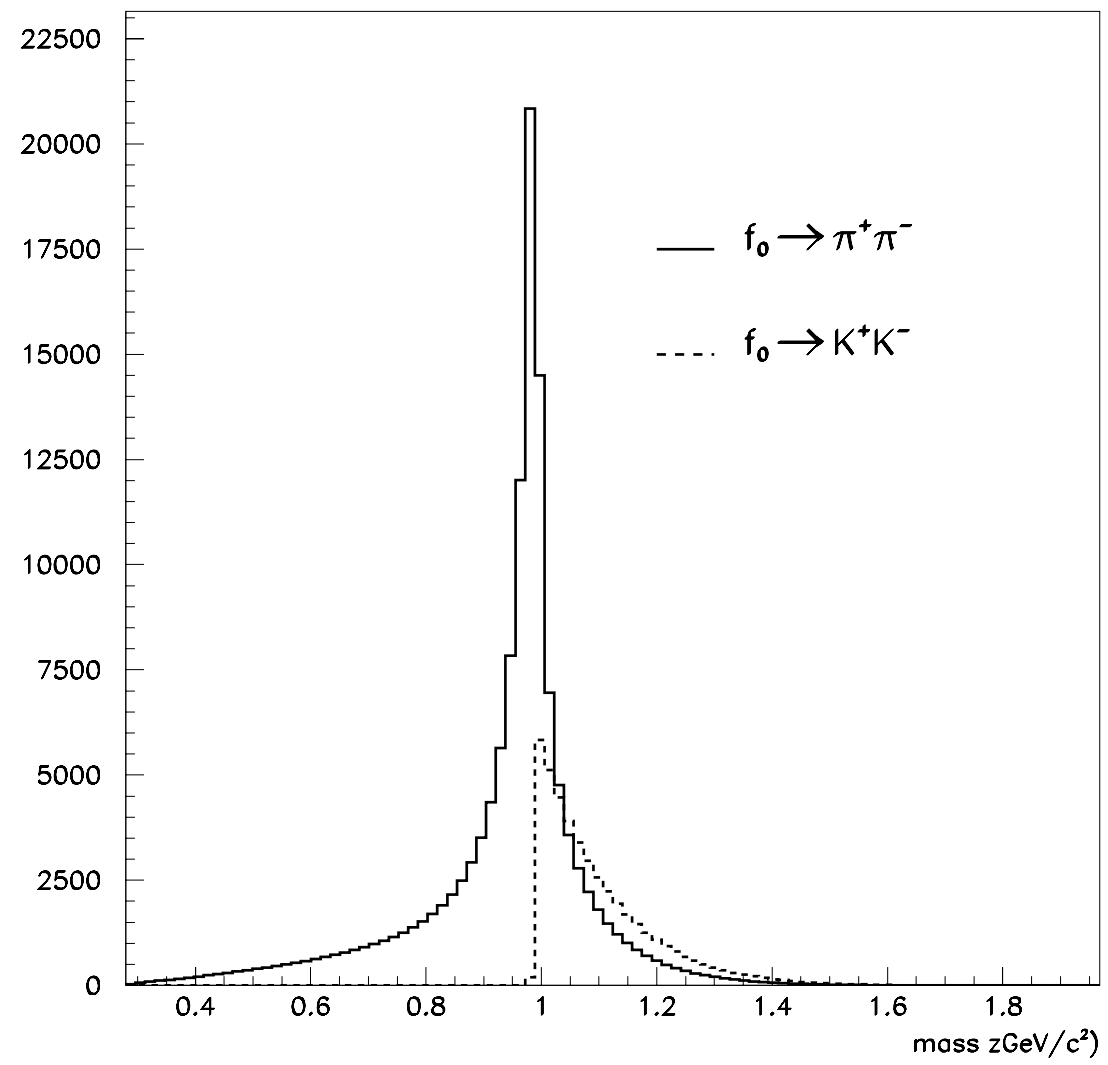

Figure 9.11: Expected $\pi^{+} \pi^{-}$and $K^{+} K^{-}$mass distributions in the decay $D_{s}^{+} \rightarrow f_{0} e^{+} \nu_{e}$. 


\section{Conclusion}

In this thesis, we have presented a study of the $D_{s}^{+} \rightarrow K^{+} K^{-} e^{+} \nu_{e}$ decay channel using a data sample of $214 \mathrm{fb}^{-1}$ recorded by the BABAR experiment. Semileptonic decays have been selected in continuum $c \bar{c}$ events with an untagged technique in which events are only partially reconstructed, allowing a rather important efficiency. Events have been selected in the $K^{+} K^{-}$mass range between 1.01 and $1.03 \mathrm{GeV} / \mathrm{c}^{2}$, where the $\phi$ meson is largely dominant.

The contributions of the $A_{2}$ and $V$ hadronic form factors, relative to $A_{1}$, have been measured in the semileptonic decay channel $D_{s}^{+} \rightarrow \phi e^{+} \nu_{e}$. In this measurement, pole mass expressions have been used for the $q^{2}$ dependence of the form factors.

With the following values for the pole masses: $m_{A}=2.5 \mathrm{GeV} / \mathrm{c}^{2}$ and $m_{V}=2.1 \mathrm{GeV} / \mathrm{c}^{2}$, we have obtained:

$$
r_{V}=V(0) / A_{1}(0)=1.799 \pm 0.046 \pm 0.051 \text { and } r_{2}=A_{2}(0) / A_{1}(0)=0.813 \pm 0.036 \pm 0.030
$$

These values are compatible and more accurate than previous determinations obtained using a similar ansatz for the $q^{2}$ variation of the form factors. The precision achieved on these form factors ratios is similar to the one obtained for the world average $D^{+} \rightarrow \bar{K}^{* 0} \ell^{+} \nu$ decay.

We have also studied the $q^{2}$ variation of the form factors and measured the value of the axial-vector pole mass, $m_{A}$. Present measurements have reduced sensitivity on $m_{V}$ and its value has been fixed to $2.1 \mathrm{GeV} / \mathrm{c}^{2}$. We have also searched for an $S$-wave contribution in the $K^{+} K^{-}$system, supposing that it corresponds to the $f_{0}$. The normalization of this $S$-wave is given by the parameter $r_{0}$. Fitting $r_{2}, r_{V}$ and $m_{A}$ and $r_{0}$ we have obtained:

$$
\begin{gathered}
r_{V}=V(0) / A_{1}(0)=1.868 \pm 0.061 \pm 0.079, r_{2}=A_{2}(0) / A_{1}(0)=0.763 \pm 0.072 \pm 0.062 \\
m_{A}=\left(2.30_{-0.18}^{+0.24} \pm 0.21\right) \mathrm{GeV} / \mathrm{c}^{2}, \text { and } \mathrm{r}_{0}=(15.3 \pm 2.6 \pm 1) \mathrm{GeV}^{-1}
\end{gathered}
$$

This is the first evidence of an $S$-wave component in the $D_{s}^{+}$semileptonic channel. The measured $S$-wave component corresponds, in the $K^{+} K^{-}$mass range between 1.01 and 
$1.03 \mathrm{GeV} / \mathrm{c}^{2}$, to the ratio:

$$
\frac{B R\left(D_{s}^{+} \rightarrow f_{0} e^{+} \nu_{e}\right) \times B R\left(f_{0} \rightarrow K^{+} K^{-}\right)}{B R\left(D_{s}^{+} \rightarrow K^{+} K^{-} e^{+} \nu_{e}\right)}=0.23_{-0.08}^{+0.12} \pm 0.03 \%
$$

Finally we have measured the branching fraction $D_{s}^{+} \rightarrow K^{+} K^{-} e^{+} \nu_{e}$ relative to the $D_{s}^{+} \rightarrow K^{+} K^{-} \pi^{+}$decay channel:

$$
R_{D s}=\frac{B R\left(D_{s}^{+} \rightarrow K^{+} K^{-} e^{+} \nu_{e}\right)^{\Delta m_{K K}}}{B R\left(D_{s}^{+} \rightarrow K^{+} K^{-} \pi^{+}\right)^{\Delta m_{K K}}}=0.5535 \pm 0.0064 \pm 0.0142
$$

from wich we obtain:

$$
A_{1}(0)=0.605 \pm 0.012 \pm 0.018 \pm 0.018
$$

These results have been compared with previous experimental measurements, when available, and theoretical calculations. Our results are compatible with those obtained by FOCUS on the form factors normalization parameters $r_{2}$ and $r_{V}$, and we are much more precise. We find a value of $r_{2}$ compatible with the one obtained for the $D^{+} \rightarrow \bar{K}^{* 0} \ell^{+} \nu$ decay, but the agreement on $r_{V}$ is more marginal. On the theoretical side, the more recent lattice QCD calculations are not in agreement with our determination of $r_{V}$ and the values of $r_{2}$ differ by less than $2 \sigma$. These calculations have been obtained using the quenched approximation and it would be of great interest to have unquenched results in order to validate the lattice calculations. 


\section{Bibliography}

[1] S.L. Glashow, Nucl. Phys. 22579 (1961);

S. Weinberg, Phys. Rev. Lett. 191264 (1967);

A. Salam, in Proc. 8th Nobel Symp., ed. N. Swartholm, Almquist and Wiksells, Stockholm (1968).

[2] N. Cabibbo, Phys. Rev. Lett. 10531 (1963).

[3] M. Kobayashi and T. Maskawa, Prog. Th. Phys. 49652 (1973).

[4] Particle Data Group, W.-M. Yao et al., Journal of Physics G 33 (2006) 1.

[5] L. Wolfenstein, Phys. Rev. Lett. 51 (1983) 1945;

A.J. Buras, M.E. Lautenbacher and G. Ostermaier, Phys. Rev. D50 (1994) 3433.

[6] A. J. Buras, M. E. Lautenbacher and G. Ostermaier, Phys. Rev. D 50, 3433 (1994).

[7] T. Inami and C.S. Lim,Prog. Theor. Phys. 65 (1981) 297; ibid. 65 (1981) 1772.

[8] G. Buchalla, A. J. Buras e M. E. Lautenbacher, Rev. Mod. Phys. 68 (1996) 1125 [hep-ph9512380].

[9] A.J. Buras, M. Jasmin and P.H. Weisz, Nucl. Phys. B 347 (1990) 491.

[10] S. Herrlich and U. Nierste, Nucl. Phys. B 419 (1994) 192.

[11] M. Bona et al.,UTfit Collaboration; [arXiv:hep-ph/0606167]. http://utfit.org

[12] J. Charles et al.,CKMfitter Collaboration, Eur. Phys. J. C41, 1-131 (2005), http://ckmfitter.in2p3.fr/ .

[13] J. D. Richman and P. R. Burchat, Rev. Mod. Phys. 67893 (1995).

[14] M. Wirbel,B. Stech and M. Bauer Z Phys. C29 637 (1985).

[15] M. Neubert, Phys. Reports 245259 (1994). 
[16] F. J. Gilman and R. L. Singleton, Jr., Phys. Rev. D41 142 (1990).

[17] J.M. Link et al.,FOCUS Collaboration, Phys. Lett. B535, 43 (2002).

[18] J.M. Link et al.,FOCUS Collaboration, Phys. Lett. B621, 72 (2005).

[19] M.R. Shepherd et al.,CLEO Collaboration, Phys. Rev. D74, 052001 (2006).

[20] J.M. Link et al., FOCUS Collaboration, Phys. Lett. B586 (2004) 183; [hep-ex/0401001].

[21] C. L. Y. Lee, M. Lu and M. B. Wise, Phys. Rev. D46 5040 (1992).

[22] A. Pais and S. B. Treiman, Phys. Rev. 1681858 (1968).

[23] M. Ablikim et al., BES Collaboration, Phys. Lett. B607 243 (2005).

[24] N. Isgur and M.B. Wise, Phys. Lett. B232 113 (1989). B237 527 (1990);

[25] B. Aubert et al.,Babar collaboration, submitted to Phys. Rev. D, [arXiv:0705.4008].

[26] I. Caprini, L. Lellouch and M. Neubert, Nucl. Phys. B 530153 (1998).

[27] J. F. Amundson and J. L. Rosner, Phys. Rev. D47 1951 (1993).

[28] M.J. dugan and B.Gristein, Phys Lett. B 255, 583 (1991).

[29] J. Charles, A. Le Yaouanc, L. Oliver, O. Péne, and J.-C. Raynal; Phys. Rev. D60 014001 (1999).

[30] C. G. Boyd, B. Grinstein, and R.F. Lebed, Phys. Lett. B353 306 (1995), Nucl. Phys. B461 493 (1996).

[31] C. G. Boyd and, M. J, Savage, Phys. Rev. D56 303 (1997).

[32] N. Isgur, D. Scora, B. Grinstein and M. B. Wise, Phys. Rev. D39, 799 (1989).

[33] D. Scora and N. Isgur, Phys. Rev. D52, 2783 (1995).

[34] T. M. Aliev, V. L. Eletsky and Y. I. Kogan, Sov. J. Nucl. Phys. 40, 527 (1984); P. Ball, V. M. Braun and H. G. Dosch, Phys. Rev. D44, 3567 (1991).

[35] T. Altomari and L. Wolfenstein, Phys. Rev. D37 691 (1988).

[36] W. Jaus, Phys. Rev. D41 3394 (1990); ibid D53 1349 (1996).

[37] R. N. faustov, V. O. Galkin and A. Yu. Mishurov, Phys. Rev. D53 1391 (1996).

[38] D. Melikhov and B. Stech, Phys. Rev. D62 014006 (2000). 
[39] D.S Du, J,W Li and A,M-Z Yang, Eur. Phys. J. C37, 173 (2004); [hep-ph/0308259].

[40] T.M Aliev, A. Ozpineci and M. Savci (2004); [hep-ph/0401181].

[41] K. G. Wilson, Phys. Rev. D10, 2445 (1974).

[42] E.D Freeland et al.,MILC collaboration; [arXiv:0710.4339].

[43] J. Gill for the UKQCD collaboration, Nucl.Phys.Proc.Suppl.106:391-393 (2002); [heplat/0109035].

[44] C. R. Allton et al.,APE collaboration, Phys.Lett.B345:513-523 (1995); [heplat/9411011].

[45] A. Abada et al.,SPQcdR collaboration, Nucl. Phys. Proc. Suppl. 119625 (2003); [heplat/0209116].

[46] B. Aubert et al. (BABAR Collaboration), Phys. Rev. D76 052005 (2007).

[47] D .Becirevic and A. B. Kaidalov, Phys. Lett. B478, 417 (2000).

[48] S.Fajfer and J. Kamenik, Phys. Rev. D72 (2005) 034029; [hep-ph/0506051] and [hep$\mathrm{ph} / 0601028]$.

[49] B. Aubert et al.,BABAR Collaboration, Nucl. Instr. and Methods A 479, 1 (2002).

[50] PEP II - An Asymmetric B Factory, Conceptual Design Report, SLAC-418, LBL-5379 (1993).

[51] P.F. Harrison, ed. et al.,BABAR Collaboration, The BABAR physics book: Physics at an asymmetric B factory., SLAC-R-0504 (1998).

[52] B. Aubert et al.,BABAR Collaboration, The First Year of the BABARexperiment at PEPII, BABAR-CONF-00/17, Contribution to $X X X^{\text {th }}$ International Conference of High Energy Physics, Osaka (Japan) (2000).

[53] M. H. Kelsey et al.,Nucl. Instr. and Method A 536, 206 (2004).

[54] I. Adam et al.,SLAC-PUB-8783 (2001).

[55] B. Lewandowski et al., Nucl. Instr. and Method A 494, 302 (2002).

[56] F. Anulli et al.,Nucl. Instr. and Method A 409, 542 (1998).

[57] P. Billior, Nucl. Instr. and Method A 225, 225 (1984).

[58] http://www.slac.stanford.edu/BFROOT/www/Physics/Tools/Pid/Selectors/r18a/selectors.html. 
[59] T. Brandt, "Likelihood based electron identification", BABAR analysis document \#391.

[60] L. Widhalm et al., BELLE collaboration, Int. J. Mod. Phys. A21 (2006) 5449; [hepex/060409].

[61] G. C. Fox and S. Wolfram, Nucl. Physs B149 (1979) 413.

[62] http://tmva.sourceforge.net/

[63] P. Abreu et al., DELPHI Collaboration, Phys. Lett. B449 364 (1999).

[64] K. Ackerstaff et al., OPAL Collaboration, E. Phys. J. C4 364 (1998).

[65] R. Barate et al., ALEPH Collaboration, Phys. Rept. 2941 (1998).

[66] P. Abreu et al., DELPHI Collaboration, Z. Phys. C73 61 (1996).

[67] V. Uvarov, Phys. Lett. B511 136 (2001).

[68] S. Dobbs et al., CLEO Collaboration, [arXiv:0712.1020].

[69] T.C. André, Annals Phys. 322 (2007) 2518; T. Alexopoulos et al., Phys. Rev. D71, 013001 (2005).

[70] J. Alexander et al., CLEO Collaboration, [arXiv:0801.0680].

[71] J.M Link et al., FOCUS Collaboration, Phys. Lett.B607 67 (2005); [hep-ph/0510053].

[72] A. Massafferri et al., FOCUS Collaboration, Nucl.Phys. Proc. Suppl.164 101 (2007); [hep-ph/0510053].

[73] K. Kodama et al., E653 Collaboration, Phys. Lett. B309 (1993) 483.

[74] P. L. Frabetti et al., E687 Collaboration, Phys. Lett. B328 (1994) 187.

[75] P. Avery et al. , CLEO Collaboration, Phys. Lett. B337 (1994) 405.

[76] E.M. Aitala et al., E791 Collaboration, Phys. Lett. B450 (1999) 294; [hep-ex/9812013].

[77] N. E. Adam et al., CLEO Collaboration, Phys. Rev. Lett. 97251801 (2006). 


\section{Remerciements}

Je tiens tout d'abord à remercier Bernard d'Almagne et Guy Wormser de m'avoir acueillie au laboratoire de l'accélérateur Linéaire pendant ces trois années. Je remercie également Guy Wormser d'avoir présidé mon jury de thèse.

Je remercie Sylvie Rosier-Lees et Vera Luth d'avoir accepté le rôle de rapporteur (rapporteuse en l'occurence). Je remercie particulièrement Vera pour son investissement dans le suivi de mon travail au sein du working group et pour ses nombreuses suggestions concernant l'analyse et le manuscript. Merci également à Olivier Pène et Philippe Schwemling d'avoir accepté de faire partie de mon jury de thèse.

Un grand merci à mon directeur de thèse, Patrick Roudeau, sans qui je ne serais jamais arrivée à un tel résultat. Ce fut un plaisir de travailler avec toi, merci de m'avoir apporté tes innombrables connaissances physiques, ta rigueur et tes encouragements. Merci également pour ton amitié, et merci d'avoir supporté Ségolène...

Je souhaiterais remercier les membres du groupe Babar du LAL: Achille, Marie Hélène, Anne Marie, Michel, Nicolas, Gilbert, Viola, Joao, Wenfeng, Lianliang, Denis et Bogdan. Vous m'avez apporté de nombreuses connaissances scientifiques et techniques, beaucoup de conseils et aussi votre amitié. Ces trois années passées avec vous ont été réellement enrichissantes. Merci Achille pour tout ce que l'on a partagé, les discussions de physique (quelquefois), de politique (surtout!), et pour ton soutien. Merci à Marie Hélène sans qui j'aurais peut être fait une thèse sur la bioluminescence du plancton méditerranéen (sujet pourtant intéressant). Je remercie également Stephane Pruvot qui m'a beaucoup aidée pendant ma première année de thèse pour mettre en place le fit. Merci aussi à Arantza pour son aide tout au long de ma thèse et pour sa contribution à ce manuscrit, merci d'être venue! Thank you Nicolas de m'avoir appris tous les secrets du DIRC et de la Wells Fargo! Merci également à Eli pour le data quality et ses conseils (sur le choix des pastèques). Bien que je ne l'ai jamais rencontré, je remercie également Jean-Yves Nief, qui a résolu bien de mes 
problèmes au ccin2p3.

Je voudrais remercier les théoriciens du LPT d'avoir participé à nos discussions: Sébastien Descotes Genon, Damir Becirevic, Alain Le Yaouanc, Olivier Pène et Benjamin Haas. Votre aide a été trés enrichissante et surtout très utile pour la compréhension des facteurs de forme, des interférences et de la QCD sur réseau.

Je voudrais remercier les services administratifs du laboratoire, ainsi que le service informatique, sans oublier le service mission, pour leur aide.

Ma thèse n'aurait pas été la même sans les jeunes du LAL et leur enthousiasme: Yasmine, Francesco, Dimitri, Joao, Mathieu, Christophe, Iro, Nikola et tous ceux que j'oublie...merci pour les bonnes soirées partagées! Et surtout, ma thèse n'aurait pas été la même sans Viola. En effet, je n'aurai sans doute pas traversé la Mongolie ni la Death Valley, et encore moins l'île de Pâques (enfin... ca reste encore à faire). Merci pour cette profonde amitié, grazie per tutto!

Je voudrais remercier également le reste de la communauté babariste nationale et internationale: italiens, espagnols, allemands, américains, anglais, canadiens..., avec qui j'ai partagé la vie californienne. Merci surtout à Enrico, Jesko et Antonio d'avoir accepté de se jeter avec moi d'un avion à $4500 \mathrm{~m}$ d'altitude.

J'en profite pour remercier ceux qui ont eu la bonne idée de me faire monter dans l'avion, les "magistèriens": Maria, Fabio \& Steph, Pascale, FX, Xav \& Marie, Marine, Julien, Miguel, Li-hua... c'etait vraiment cool de vous rencontrer en débarquant à Paris, c'est peut être grâce à vous que j'y suis encore. Et comme dirait Pascale, c'est moi la "prem's", donc maintenant c'est à vous...bon courage!

Une "spécial dédicace" à mon amie de toujours, ma soeur, Julie (alias Dawta Jena) avec qui je partage toutes les bonnes vibes de la vie depuis 18 ans. Merci à Anouch et la famille Nersessian. Merci d'avoir toujours été là, même si je suis partie dans le Nord lointain! Jah Love!

Enfin, cette thèse n'aurait jamais été possible sans ma famille. Merci à mes parents de m'avoir toujours laissé la liberté, et de m'avoir toujours donné les moyens de faire ce que je désirais. Je remercie aussi mes grands-parents, Corinne, Claude, Steph et Van, et Gaspard, pour leur soutien si important. Merci pour votre amour. 